
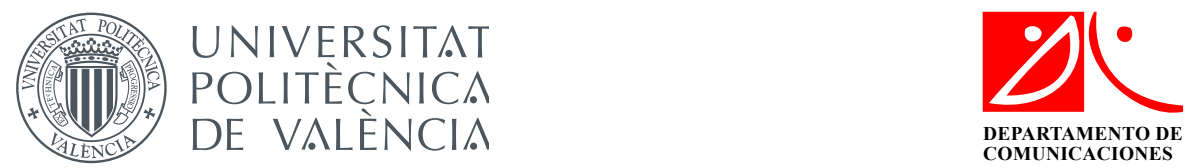

\title{
Distributed Cooperative MIMO in Beyond 2020 Wireless Networks
}

\author{
Departamento de Comunicaciones \\ Universitat Politècnica de València
}

\author{
A thesis submitted for the degree of \\ Doctor por la Universitat Politècnica de València \\ Valencia, February 2016
}

Author:

Jorge Cabrejas Peñuelas

Supervisors:

Dr. José F. Monserrat del Río Dr. Narcís Cardona Marcet 

A mis padres, a mis hermanas y a Merche. 



\section{Abstract}

Mobile communication systems are currently being developed with the aim of providing peak data rates up to 20 times higher to those of Long Term Evolution (LTE)-Advanced Release 10. However, this performance improvement is often far from being the experimented performance by all users, especially, for those users who are far from the base station. In this sense, there exists a consensus within the international scientific community on the fact that the best way to achieve the same quality for all users is with the use of heterogeneous networks composed of macrocells, microcells, femtocells, and relays.

This dissertation addresses the use of mobile relays to provide service to users who are out-of-coverage or undergo low data rates as they are located at the cell-edge. Mobile relaying is a natural extension of the fixed relay in which users who are in the idle state could retransmit signals received from other transmitters to enhance signal quality and consequently data rates. The use of mobile relaying could make the data boost required by the future Fifth Generation (5G) affordable.

Our investigations employ a simulation platform that includes link-level and system-level simulations. This dissertation focuses on proposing and evaluating new techniques that manage the use of the mobile relay in the new generation cellular networks. In particular, the dissertation studies mobile relaying from two complementary points of view.

The first point of view investigates the mobile relay management at the network level through a signaling protocol known as Media Independent Handover (MIH). The main idea of the proposed mechanism is to use this signaling to connect the base station and the user in one of the following two manners. In the former, both entities are connected directly through the $\mathrm{xG}(\mathrm{x}=2,3,4$, 5) wireless network. In the latter, there exists an $\mathrm{xG}$ connection between the base station and the mobile relay and another one between the mobile relay and the user through an IEEE 802.11 local wireless network. The investigations in this Thesis aim at finding a trade-off between using multiple mobile relays and reducing signaling overhead. 


\section{ABSTRACT}

The second point of view deals with mobile relying integration at the air interface level. It consists in detecting, proposing, and evaluating new transmission techniques that solve the drawbacks derived from coherent detection. As with point-to-point systems, employing multiple antennas in a cooperative system can significantly improve the spectral efficiency of the systems with only one transmit antenna assuming that the channel state information is available at the receiver. However, performing a coherent detection in a network assisted by relays consumes much more resources than a point-to-point network since the coherent detection requires the channel estimation of source-relay, relaydestination, and source-destination links. In this Thesis, the proposed solution is to use transmission techniques that do not need the channel knowledge to perform the detection. This transmission mode is referred to as non-coherent.

This dissertation evaluates, firstly, the use of single-user open-loop communication techniques over temporally-correlated Rayleigh fading Multiple Input Multiple Output (MIMO) channels. Two non-coherent techniques are compared with several coherent schemes, for two and four antennas. On the other hand, in multi-carrier systems, the dissertation proposes to transmit the Grassmannian signaling in the virtual block formed by the coherence time and the coherence bandwidth. This proposal is due to the fact that Grassmannian signaling achieves data rates approaching capacity over block-fading channels where the channel coefficients are constant during $T$ time slots. However, this channel type is not common in real systems since channel correlation is often found in frequency, time, and space. For this reason, the next objective is to evaluate the performance of Grassmannian signaling compared to the diversity transmission modes of LTE, analyzing the impact of user mobility and antenna correlation. Thanks to these investigations, we point that noncoherent systems are promising techniques in mobility scenarios with a high number of transmit antennas. This result motivates its relevance in the design of new single-user open-loop transmission methods with multiple antennas. In downlink multi-user non-coherent scenarios, superposition coding and a new suboptimum detection scheme are proposed. This detection system considerably reduces the complexity respect to the maximum likelihood detection. Finally, this dissertation proposes that Grassmannian signaling is transmitted in a new carrier type, where any reference signal is transmitted if it is indicated in the signaling forwarded to the user. In this way, the user would change its detection method to non-coherent. 


\section{Resumen}

Los sistemas de comunicaciones móviles están siendo desarrollados en la actualidad con el objetivo de proporcionar tasas de datos de pico de hasta 20 veces superiores a las proporcionadas por Long Term Evolution (LTE)-Advanced Release 10. Sin embargo, esta mejora en las prestaciones a menudo dista mucho de ser la experimentada por todos los usuarios, sobre todo por aquellos que están lejos de la estación base. En este sentido, existe un consenso entre la comunidad científica internacional sobre que la forma más eficiente de conseguir la misma calidad de servicio para todos los usuarios es con el uso de redes heterogéneas que incluyan macroceldas, picoceldas, femtoceldas y relays.

Esta Tesis aborda el uso de relays móviles para dar servicio a usuarios que se encuentran fuera de cobertura o que experimentan bajas tasas de datos debido a que están situados en el borde de la celda. El relay móvil es una extensión natural del relay fijo, de manera que usuarios que estén en reposo podrían retransmitir las señales recibidas de otros transmisores, mejorando así el nivel de señal recibido y por consiguiente la tasa experimentada. Esto permitiría mejorar la tasa del sistema hasta alcanzar los requisitos de la 5G con un coste razonable.

Las investigaciones de esta Tesis doctoral emplean una plataforma de simulación que incluye simulaciones a nivel de enlace y a nivel de sistema. Esta Tesis se centra en proponer y evaluar nuevas técnicas que gestionen el uso del relay móvil en las redes celulares de nueva generación. En particular, esta Tesis propone la gestión del relay móvil desde dos puntos de vista.

El primer punto de vista se centra en la gestión del relay móvil a nivel de red a través de una señalización conocida como Media Independent Handover (MIH). La idea principal del mecanismo propuesto es usar esta señalización para conectar la estación base y el usuario de dos formas diferentes. En la primera alternativa, ambas entidades están conectadas directamente a través de la red inalámbrica $\mathrm{xG}(\mathrm{x}=2,3,4,5)$. En la segunda alternativa, existe una conexión $\mathrm{xG}$ desde la estación base al relay móvil, y otra desde el relay móvil al usuario a través de una red local inalámbrica IEEE 802.11. Las 


\section{RESUMEN}

investigaciones de esta Tesis se centran en encontrar un compromiso entre usar multiples relays móviles y reducir al máximo la carga de señalización.

El segundo punto de vista se centra a nivel radio y consiste en proponer y evaluar nuevas técnicas de transmisión que solucionen los problemas derivados de la detección coherente. Al igual que ocurre con los sistemas punto a punto, emplear múltiples antenas en un sistema cooperativo puede mejorar significativamente la eficiencia espectral respecto a los sistemas con una única antena transmisora con la condición de que la información del estado del canal esté disponible en el receptor. Sin embargo, realizar una detección coherente en una red con relays consume muchos más recursos que en una red punto a punto, puesto que la detección coherente requiere la estimación del canal de los enlaces fuente-relay, relay-destino y fuente-destino. En esta Tesis, la solución propuesta es usar técnicas de transmisión que no necesiten el conocimiento del canal para hacer la detección. Este modo de transmisión se conoce como no coherente.

Esta Tesis evalúa, en primer lugar, el uso de técnicas de comunicación en lazo abierto y a un único usuario sobre canales Multiple Input Multiple Output (MIMO) con desvanecimientos Rayleigh temporalmente correlados. Se comparan dos técnicas no coherentes con varios esquemas coherentes, para dos y cuatro antenas. Por otra parte, en sistemas multiportadora, la Tesis propone transmitir la señalización Grassmannian en el bloque virtual formado por el tiempo de coherencia y el ancho de banda de coherencia. Esto se debe a que la señalización Grasmannian alcanza tasas próximas a la capacidad en canales block-fading donde los coeficientes del canal son constantes durante $T$ periodos de tiempo. Sin embargo, este tipo de canal no suele ser común en sistemas reales, puesto que la correlación del canal suele encontrarse en frecuencia, en tiempo y en espacio. Por este motivo, el objetivo siguiente consiste en evaluar las prestaciones de la señalización Grassmannian con los modos de transmisión de diversidad de un sistema celular como LTE, analizando el impacto de la movilidad y la correlación de las antenas. Gracias a estos estudios, se muestra que los sistemas no coherentes son técnicas prometedoras en escenarios con movilidad y con un alto número de antenas. Esto motiva su relevancia en el diseño de nuevas técnicas de transmisión para un sólo usuario, en lazo abierto y con múltiples antenas. En escenarios multiusuario no coherente, esta Tesis propone el uso de superposition coding y un esquema de detección subóptimo en el enlace descendente. Este esquema de detección reduce considerablemente la complejidad respecto a la detección de máxima verosimilitud. Finalmente, esta Tesis propone que la señalización Grassmannian sea transmitida en un nuevo tipo de portadora en la que ninguna señal de referencia se transmita si así se indica en la señalización transmitida al usuario. De esta forma, el usuario cambiaría su forma de detectar a no coherente. 


\section{Resum}

Els sistemes de comunicacions mòbils estan sent desenrotllats en l'actualitat, amb l'objectiu de proporcionar taxes de pic de fins a 20 vegades superiors a les proporcionades per Long Term Evolution (LTE)-Advanced Release 10. No obstant això, aquest creixement en prestacions sovint està lluny de ser l'experimentat per tots els usuaris, sobretot per aquells que estan lluny de l'estació base. En este sentit, hi ha un consens entre la comunitat científica internacional en què la forma més eficient d'aconseguir la mateixa qualitat de servici per a tots els usuaris és amb l'ús de xarxes heterogènies formades per macrocel·les, picocel-les, femtocel-les i relays.

Esta Tesi aborda l'ús de relays mòbils per a donar servici a usuaris que es troben fora de cobertura o que són servits amb baixes taxes de dades pel fet que es troben en el límit de la cel·la. El relay mòbil és una extensió natural del relay fix, de manera que, usuaris que estiguen en repòs podrien retransmetre els senyals rebuts d'altres transmissors, incrementant en nivell de senyal i per tant la taxa de dades. Açò suposaria gaudir de la velocitat que supossa la 5G amb un cost raonable.

La investigació en esta Tesi utilitza una plataforma de simulació que inclou simulacions a nivell d'enllaç i a nivell de sistema. Esta Tesi es centra a localitzar, proposar i avaluar noves tècniques que gestionen l'ús del relay mòbil en les xarxes cel-lulars de nova generació. En particular, la Tesi proposa la gestió del relay mòbil des de dos punts de vista.

El primer punt de vista es centra en la gestió del relay mòbil a nivell de xarxa a través d'una senyalització coneguda com Media Independent Handover (MIH). La idea principal del mecanisme proposat és usar esta senyalització per a connectar l'estació base i l'usuari de dos formes possibles. En la primera forma, ambdós entitats estan connectades directament a través de la xarxa sense fil $\mathrm{xG}(\mathrm{x}=2,3,4,5)$. En la segona forma, hi ha una connexió $\mathrm{xG}$ des de l'estació base al relay mòbil, i una altra des del relay mòbil a l'usuari a través d'una xarxa local sense fil IEEE 802.11. Les investigacions es centren a 
trobar un compromís entre usar múltiples relays mòbils i minimitzar la càrrega de senyalització.

El segon punt de vista es centra a nivell ràdio i consistix a proposar i avaluar noves tècniques de transmissió que solucionen els problemes derivats de la detecció coherent. Igual que ocorre amb els sistemes punt a punt, emprar múltiples antenes en un sistema cooperatiu pot millorar significativament l'eficiència espectral respecte als sistemes amb una única antena transmissora amb la condició que la informació de l'estat del canal estiga disponible en el receptor. No obstant aixó, realitzar una detecció coherent en una xarxa amb relays consumix molts més recursos que en una xarxa punt a punt, ja que la detecció coherent requerix l'estimació del canal dels enllaços font-relay, relay-destí i font-destí. En esta Tesi, la solució proposada és usar tècniques de transmissió que no necessiten el coneixement del canal per a fer la detecció. Este mode de transmissió es coneix com no coherent.

Esta Tesi avalua, en primer lloc, l'ús de tècniques de comunicació en llaç obert i a un únic usuari sobre canals Multiple Input Multiple Output (MIMO) amb esvaïments Rayleigh temporalment correlats. Dos tècniques no coherents han sigut comparades amb diversos esquemes coherents, per a dos i quatre antenes. D'altra banda, en sistemes multiportadora, la Tesi proposa transmetre la senyalització Grassmannian en el bloc virtual format pel temps de coherència i l'amplada de banda de coherència. Açò es degut al fet que la senyalització Grasmannian aconseguix taxes pròximes a la capacitat en canals block-fading on els coeficients del canal són constants durant $T$ períodes de temps. No obstant això, este tipus de canal no sol ser comú en sistemes reals, ja que la correlació del canal sol trobar-se en freqüència, en temps i en espai. Per este motiu, el següent objectiu és avaluar les prestacions de la senyalització Grassmannian amb els modes de transmissió de diversitat d'un sistema cel-lular com LTE, analitzant l'impacte de la mobilitat i la correlació de les antenes. Gràcies a estos estudis, mostrem que els sistemes no coherents són unes tècniques prometedores en escenaris amb mobilitat i alt nombre d'antenes. Açò motiva la seua rellevància en el disseny de noves tècniques de transmissió per a un únic usuari, en llaç obert i amb múltiples antenes. En escenaris multiusuari no coherent, es proposa l'ús de superposition coding i un esquema de detecció subòptim en l'enllaç descendent. Este esquema de detecció reduïx considerablement la complexitat respecte a la detecció de máxima versemblança. Finalment, esta Tesi proposa que la senyalització Grassmannian siga transmesa en un nou tipus de portadora en la que cap senyal de referència és transmesa si això és indicat en la senyalització transmesa a l'usuari. D'esta manera, l'usuari canviaria la seua forma de detectar a no coherent. 


\section{Acknowledgements}

I would like that these lines help to thank those who made this Thesis possible. First and foremost, I would like to thank my supervisors. Thanks to Professor Narcís Cardona for giving me the opportunity of being part of the Mobile Communications Group (MCG) of the Institute of Telecommunications and Multimedia Applications (iTEAM) at the Universitat Politècnica de València (UPV). I would like to express my gratitude to Dr. José F. Monserrat because he has been the principal source of encouragement and knowledge in all these years; without him, this Thesis would have never been written.

It is also a pleasure to thank Professor Halim Yanikomeroglu for inviting me to visit his exceptional research team at Carleton University, Ottawa (Canada). I could discover the complicated Grassmannian world and meet nice people like Dr. Ramy Gohary, Dr. Rainer Schoenen, and Tamer Beitmal. I also made great friends like Yasser Fouad, who made me feel at home and helped me in everything he could.

I would like to thank especially David Martín-Sacristán, first, for him great friendship, and secondly, for his continued support for this Thesis. He also encouraged me when I did not believe in me. I cannot forget the Astellia group, Irene and Vicente, through which I have learned the practical vision of mobile communication systems. They have encouraged me significantly in my daily life with the Thesis. I would also like to thank the Grassmannian group at the UPV, Dr. Sandra Roger and Dr. Daniel Calabuig for their valuable discussions and comments in the preparation of the papers.

I want to express my gratitude to other friends who have shared my life and given me good moments all these years. I am grateful to Jordi, Charlie, Pedro, Sonia, Miguel, Pablo, Jaime, Jose, David Vargas, Jonas, Juanan, Tere, David García, Conchi, Alicia, Jordi Joan, Gerardo, Edu, Manuel, Carlos, Alejandro, and many others.

Muchas gracias a mis padres, Maria Dolores y Teodoro, y a mis hermanas Maria, Ana y Vero, por su ayuda incondicional. Me gustaría dedicar esta Tesis a la memoria de mi abuelo Teófilo que fue un ejemplo para mí. 


\section{ACKNOWLEDGEMENTS}

Finalmente, y con mucho cariño, me gustaría agradecer a Merche por todo su amor y paciencia con la Tesis. Ella es la única persona que sabe qué siento al terminar esta Tesis. Sin ella este trabajo no podría haberse llevado a cabo. Gracias por todo.

Jorge Cabrejas Peñuelas

Valencia, February 2016 


\section{Table of contents}

List of acronyms xiii

1 Introduction $\quad 1$

1.1 Background ........................... 1

1.1.1 Evolution from $4 \mathrm{G}$ towards $5 \mathrm{G} \ldots \ldots . . . . . . . .1$

1.1.2 $5 \mathrm{G}$ standardization process ........... . . 3

1.2 State of the art analysis . . . . . . . . . . . . . . . . . . . 4

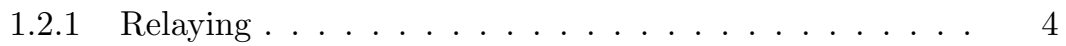

1.2 .2 D2D communications .............. 8

$1.2 .3 \mathrm{MIH} \ldots \ldots . \ldots . \ldots 9 . \ldots . \ldots 9$

1.2.4 Coherent space-time schemes ........... . . 10

1.2.5 Non-coherent space-time schemes . . . . . . . . . . 10

1.3 Problem formulation . . . . . . . . . . . . . . . . . 12

1.4 Objectives and Thesis scope . . . . . . . . . . . . . . . 13

1.5 Research methodology . . . . . . . . . . . . . . . 14

1.6 Thesis outline and main contribution . . . . . . . . . . . . 15

1.6.1 Chapter 2 - Features and challenges of the cooperative 5 G schemes ................ . 16

1.6.2 Chapter 3 - Network-layer solution: $\mathrm{MIH}$ for mobile relaying .................. . . 16

1.6.3 Chapter 4 - Radio solution: non-coherent communications schemes . . . . . . . . . . . 17

1.7 List of publications . . . . . . . . . . . . . . . . . . . 19

2 Features and challenges of the cooperative 5G schemes 23

2.1 Introduction . . . . . . . . . . . . . . . 23

2.2 Fixed relay ..................... 24

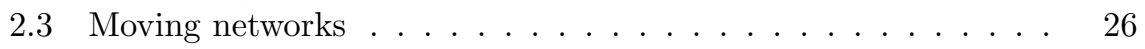

2.4 D2D communications . . . . . . . . . . . . 28 
2.4 .1 Scenarios . . . . . . . . . . . . . . . . . . 29

2.4.2 Evaluation methodology, metrics and channel modeling 29

2.4 .3 Physical layer aspects . . . . . . . . . . . . . . 30

2.4 .4 Discovery . . . . . . . . . . . . . . . . . . . . . . 32

2.4.5 Direct communication . . . . . . . . . . . . . 33

2.5 V2X communications . . . . . . . . . . . . . . 34

2.5.1 Traditional vehicular protocols . . . . . . . . . . 34

2.5.2 Communication types . . . . . . . . . . . . . 35

2.5 .3 Use cases $\ldots \ldots \ldots \ldots \ldots$

2.5 .4 Design . . . . . . . . . . . . . . . . . 36

2.6 Discussion and future trends $\ldots \ldots \ldots \ldots \ldots$

3 Network-layer solution: MIH for mobile relaying 39

3.1 Introduction . . . . . . . . . . . . . . . . . . . . 39

3.2 Current technological scenario . . . . . . . . . . . . . . 40

3.2 .1 LTE-Advanced . . . . . . . . . . . . . . . . . . . . . 41

$3.2 .2 \quad$ IEEE $802.11 \mathrm{n} \ldots \ldots \ldots \ldots \ldots$. . . . . . . . . . . . 41

$3.2 .3 \quad$ МIH . . . . . . . . . . . . . . . . . . . . . . . 41

3.2 .4 Localization . . . . . . . . . . . . . . . . . . . . . 42

3.3 MIH-driven relay selection mechanism . . . . . . . . . . . 43

3.3 .1 Situation $1 \ldots \ldots \ldots \ldots$

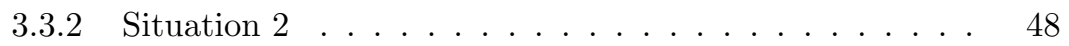

3.3 .3 Situation $3 \ldots \ldots \ldots$

3.4 Assessment methodology and system modeling . . . . . . . 50

3.5 Results and discussion . . . . . . . . . . . . . . . . 51

3.5.1 Threshold evaluation . . . . . . . . . . . . . 52

3.5.2 Performance evaluation of RDA . . . . . . . . . 55

3.5.3 Velocity impact evaluation in performance . . . . . . . 56

3.5.4 Deployment cost in an LTE-Advanced system . . . . . . 56

3.6 Conclusions . . . . . . . . . . . . . . . . . . . . 59

4 Radio solution: non-coherent communications schemes $\quad 61$

4.1 Introduction . . . . . . . . . . . . . . . . . . 61

4.2 Pilot design in the LTE technology . . . . . . . . . . . . 63

4.2 .1 Pilot contamination . . . . . . . . . . . . 63

4.2.2 Loss of spectral efficiency and scalability problem . . . . 64

4.3 Comparison of coherent and non-coherent schemes: singlechannel MIMO systems . . . . . . . . . . . . . . . . . 64

4.3 .1 System model . . . . . . . . . . . . . . . . . . 64

4.3.2 Non-coherent schemes: DUSTM . . . . . . . . . . 65

4.3.3 Non-coherent schemes: Grassmannian codes . . . . . . . 67 
4.3.4 Coherent training-based schemes . . . . . . . . . . . 69

4.3.5 Performance analysis over time-varying channels . . . 71

4.4 Grassmannian signaling in multi-channel frequency-flat MIMO

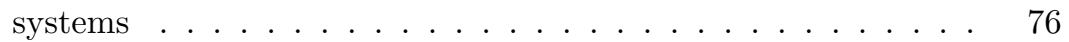

4.4.1 System model . . . . . . . . . . . . . . . . . 76

4.4.2 Degradation of independent narrowband Grassmannian signaling . . . . . . . . . . . . . . . . . 77

4.4.3 Reshaping of the transmitted pulse . . . . . . . . . 78

4.4.4 Concatenated Grassmannian signaling . . . . . . . . 78

4.4.5 Grassmannian signaling in fast fading channels . . . . 80

4.4.6 Comparison between concatenated and independent narrowband Grassmannian signaling . . . . . . . . . . . . 80

4.5 Comparison of coherent and non-coherent schemes: multichannel MIMO systems . . . . . . . . . . . . . 82

4.5.1 Frequency-time mapping of Grassmannian codes . . . . 82

4.5.2 Performance analysis over time and frequency varying spatially correlated channels . . . . . . . . . . . . 84

4.6 Multi-user MIMO transmission and reception with Grassmannian signaling . . . . . . . . . . . . . . . . . . . . . . . . . . 88

4.6 .1 System model . . . . . . . . . . . . . . . . . . . . 88

4.6.2 Non-coherent multi-user receiver . . . . . . . . . . . 89

4.6.3 Proposed non-coherent multi-user detection . . . . . . . 90

4.6.4 Downlink non-coherent multi-user gain . . . . . . . . . 92

4.6.5 Results . . . . . . . . . . . . . . . . . . 93

4.7 Integration of Grassmannian signaling for the future 5G com-

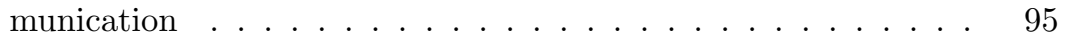

4.7.1 CA and the integration of non-coherent carriers . . . . 96

4.7.2 Effect of the imbalance among the powers received from distributed transmission points . . . . . . . . . . . . . . 99

4.8 Conclusion . . . . . . . . . . . . . . . . . . . . . 101

5 Conclusions and future work $\quad 105$

5.1 Concluding remarks . . . . . . . . . . . . . . . 105

5.2 Future research issues . . . . . . . . . . . . . . . . 107

$\begin{array}{ll}\text { Appendices } & 109\end{array}$

A Simulation tools . . . . . . . . . . . . . . . . 109

A.1 System-level simulator . . . . . . . . . . . . . . . . 109

A.1.1 IEEE 802.11n . . . . . . . . . . . . . . . 109

A.1.2 LTE . . . . . . . . . . . . . . . . . 110

A.2 Link-level simulator . . . . . . . . . . . . . . 112 
A.2.1 Inputs and outputs . . . . . . . . . . 113

A.2.2 Transport block generator and comparator . . 114

A.2.3 Transmitter . . . . . . . . . . . . . . . . 114

A.2.4 Channel . . . . . . . . . . . . . . . . . . . 119

A.2.5 Receiver . . . . . . . . . . . . 125

B Basics on non-coherent signaling . . . . . . . . . . 133

B.1 Basics on subspaces . . . . . . . . . . . . . . . 133

B.2 Optimal signal structure . . . . . . . . . . . . . 133

B.3 Preliminaries of Grassmannian signaling . . . . . . . . 134

C Signal to noise ratio calculation . . . . . . . . . . . 135

D Derivation details of Section $4.6 \ldots \ldots \ldots \ldots$

D.1 Derivation of Equation (4.23) . . . . . . . . . 137

D.2 Derivation of Equations (4.27) and (4.28) $\ldots \ldots \ldots$

References 


\section{List of acronyms}

1D

2D

2G

3G

3GPP

$4 \mathrm{G}$

$5 \mathrm{G}$

ACK

A-GNSS

AP

ARIB

ARQ

AWGN

AWS

BER

BLAST

BLER

BPSK
1-Dimension

2-Dimensions

Second Generation

Third Generation

Third Generation Partnership Project

Fourth Generation

Fifth Generation

positive ACKnowledgement

Assisted-Global Navigation Satellite System

Annuity Payment

Association for Radio Industry and Business

Automatic Repeat-reQuest

Additive White Gaussian Noise

Advanced Wireless Services

Bit Error Rate

Bell labs LAyered Space-Time

BLock Error Rate

Binary Phase-Shift Keying 
BS

CA

CAPEX

CBR

CDF

CEUSE

CEUT

$\mathrm{CoC}$

CQI

CRC

CRS

CSI

DD

DeNB

DL

DM-RS

DPSK

DUSTM

D2D

D2DSS

E-CID

eMBB

eNB

E-SMLC

ETR
Base Station

Carrier Aggregation

CAPital EXpenditures

Constant Bit Rate

Cumulative Distribution Function

Cell-Edge User Spectral Efficiency

Cell-Edge User Throughput

Component Carrier

Channel Quality Indicator

Cyclic Redundancy Check

Common Reference Signal

Channel State Information

Digital Dividend

Donor evolved Node B

Downlink

DeModulation Reference Signal

Differential Phase Shift Keying

Differential Unitary Space-Time Modulation

Device-to-Device

Device-to-Device Synchronization Signal

Enhanced-Cell ID

enhanced Mobile BroadBand

evolved Node B

Enhanced Serving Mobile Location Centre

Effective Transmission Rate 
ETSI

E-UTRAN

EVA

FCC

FDD

FEC

FER

FFT

FIR

FSTD

FTP

GC

GLRT

GNSS

HARQ

HeNB

HO

HSPA

IEEE

IFFT

IMT

IMT-2020

InH

IP

IS
European Telecommunications Standards Institute

Evolved UMTS Terrestrial Radio Access Network

Extended Vehicular A

Federal Communications Commission

Frequency Division Duplexing

Forward Error Correction

Frame Error Rate

Fast Fourier Transform

Finite Impulse Response

Frequency-Switched Transmit Diversity

File Transfer Protocol

Grassmannian Constellations

Generalized Likelihood Receiver Test

Global Navigation Satellite System

Hybrid Automatic Repeat-reQuest

Home eNodeB

HandOver

High Speed Packet Access

Institute of Electrical and Electronic Engineers

Inverse Fast Fourier Transform

International Mobile Telecommunication

International Mobile Telecommunication 2020

Indoor Hotspot

Internet Protocol

Information Server 
ISP

ITS

ITS-G5

ITU

ITU-R

QAM

LDPC

LGD

LLR

LMMSE

LoS

LPP

LPPa

LTE

LUT

L2S

MAC

MAP

MBSFN

MCS

METIS

MICS

MIDRES
Internet Service Providers

Intelligent Transportation Systems

Intelligent Transportation Systems G5

International Telecommunication Union

International Telecommunication Union

Radiocommunication

Quadrature Amplitude Modulation

Low-Density Parity Check

Link Going Down

Log-Likelihood Ratio

Linear Minimum Mean Square Error

Line of Sight

LTE Positioning Protocol

LTE Positioning Protocol annex

Long Term Evolution

Look-Up Table

Link to System

Medium Access Control

Maximum a Posteriori

Multicast-Broadcast Single-Frequency Network

Modulation and Coding Scheme

Mobile and wireless communications Enablers for Twenty-twenty (2020) Information Society

Media Independent Handover Command Services

Media Independent Handover-Driven Relay Selection mechanism 


$\begin{array}{ll}\text { MIES } & \text { Media Independent Handover Event Services } \\ \text { MIH } & \text { Media Independent Handover } \\ \text { MIHF } & \text { Media Independent Handover Function } \\ \text { MIIS } & \text { Media Independent handover Information Services } \\ \text { MIMO } & \text { Multiple Input Multiple Output } \\ \text { ML } & \text { Maximum Likelihood } \\ \text { MN } & \text { Mobile Node } \\ \text { MME } & \text { Mobility Management Entity } \\ \text { MMSE } & \text { Minimum Mean Square Error } \\ \text { mMTC } & \text { massive Machine Type Communications } \\ \text { MR } & \text { Mobile Relay } \\ \text { MRC } & \text { Maximum Ratio Combining } \\ \text { MU } & \text { Multi-User } \\ \text { MUT } & \text { Mean User Throughput } \\ \text { MU-MIMO } & \text { Multi-User MIMO } \\ \text { NACK } & \text { Negative ACKnowledgement } \\ \text { NCT } & \text { New Carrier Type } \\ \text { NLoS } & \text { Non Line of Sight } \\ \text { OFDM } & \text { Orthogonal Frequency Division Multiplexing } \\ \text { OPEX } & \text { OPex EXpenditures } \\ \text { OSTBC } & \text { Orthogonal Space-Time Block Code } \\ \text { OTDoA } & \text { Observed Time Difference of Arrival } \\ \text { PBCH } & \text { Physical Broadcast CHannel } \\ \text { PCell } & \text { Primary serving Cell } \\ \text { PCI } & \text { Physical Cell Identifier } \\ & \end{array}$


PDCCH Physical Downlink Control CHannel

PDP Power Delay Profile

PDSCH Physical Downlink Shared CHannel

PDU Protocol Data Unit

PER Packet Error Rate

PHY PHYsical

PMMLCG Primer Modulus Multiplicative Linear Congruential

Generation

PoS Point of Service

ProSe Proximity-based Services

PSK Phase-Shift Keying

PSS Primary Synchronization Signal

PUCCH Physical Uplink Control CHannel

PUSCH Physical Uplink Shared CHannel

QAM Quadrature Amplitude Modulation

QoS Quality of Service

QOSTBC Quasi-Orthogonal Space-Time Block Code

QPSK Quadrature Phase-Shift Keying

R-PDCCH Relay-Physical Downlink Control CHannel

RAN Radio Access Network

RAT Radio Access Technology

RB Resource Block

RDA Relay Discrimination Algorithm

RE Resource Element

RMa Rural Macrocell 


$\begin{array}{ll}\text { RN } & \text { Relay Node } \\ \text { RNTI } & \text { Radio Network Temporary Identifier } \\ \text { RRH } & \text { Remote Radio Head } \\ \text { RRM } & \text { Radio Resource Management } \\ \text { RRC } & \text { Radio Resource Control } \\ \text { RS } & \text { Reference Signal } \\ \text { RSU } & \text { Road Side Unit } \\ \text { SAE } & \text { Society of Automotive Engineers } \\ \text { SCell } & \text { Secondary serving Cell } \\ \text { SFBC } & \text { Space-Frequency Block Coding } \\ \text { SIB } & \text { System Information Block } \\ \text { SIMO } & \text { Single Input Multiple Output } \\ \text { SINR } & \text { Signal to Interference plus Noise Ratio } \\ \text { SISO } & \text { Single Input Single Output } \\ \text { SMa } & \text { Suburban Macrocell } \\ \text { SMN } & \text { Solicitor Mobile Node } \\ \text { SNR } & \text { Signal to Noise Ratio } \\ \text { SSS } & \text { Secondary Synchronization Signal } \\ \text { STBC } & \text { Space-Time Block Code } \\ \text { STC } & \text { Space-Time Codes } \\ \text { SU } & \text { Single-User } \\ \text { TB } & \text { Transport Block } \\ \text { TDD } & \text { Time Division Duplexing } \\ \text { TDM } & \text { Time Division Multiplexing } \\ \text { TP } & \end{array}$


TTI

TTT

UE

UL

UMa

UMi

URC

USTM

VoIP

V2I

V2N

V2P

V2V

V2X

WAVE

WiFi

WINNER

WINNER+

WiMAX

WLAN

WP
Transmission Time Interval

Time To Trigger

User Equipment

Uplink

Urban Macrocell

Urban Microcell

Ultra-Reliable and low-latency Communications

Unitary Space-Time Modulation

Voice over IP

Vehicle to Infraestructure

Vehicle to Network

Vehicle to Pedestrian

Vehicle to Vehicle

Vehicle to Everything

Wireless Access in Vehicular Environments

Wireless Fidelity

Wireless World Initiative New Radio

Wireless World Initiative New Radio +

Worldwide Interoperability for Microwave Access

Wireless Local Area Network

Working Party 


\section{Chapter 1}

\section{Introduction}

\subsection{Background}

The development of Internet has meant an unprecedented technological leap in the last twenty years. At the time of writing, 3.2 billion people frequently use Internet, compared with 400 million in 2000, which represents an increase of $800 \%$ [1]. Certainly, this growth encourages operators to invest in the development of their networks to offer a comprehensive service to users. Much of the development of Internet is due to the increase in the number of subscribers to mobile broadband, whose penetration rate reached $47 \%$ in 2015 , quadrupling the rate of fixed broadband subscriptions. In this new scenario, the aim of operators is to provide data rates similar or even superior to the traditional Internet Service Providers (ISP). Nevertheless, even though Second Generation $(2 \mathrm{G})$ and Third Generation $(3 \mathrm{G})$ networks have evolved in recent years, their users are still experiencing data rates below their expectations, especially for users who are on the cell-edge. To solve these coverage problems and boost data rates, the Third Generation Partnership Project (3GPP) proposed for its new Fourth Generation $(4 \mathrm{G})$ architecture the use of a heterogeneous network composed of macro base stations and low power nodes including pico, femto, and relay nodes.

\subsubsection{Evolution from $4 \mathrm{G}$ towards $5 \mathrm{G}$}

Once the specifications for Long Term Evolution (LTE) (Release 8) were completed, the 3GPP began to work on the LTE-Advanced standard (Release 9 and beyond). Its primary objective was to offer higher browsing speeds in a costeffective manner, and at the same time, meet or even exceed the requirements 


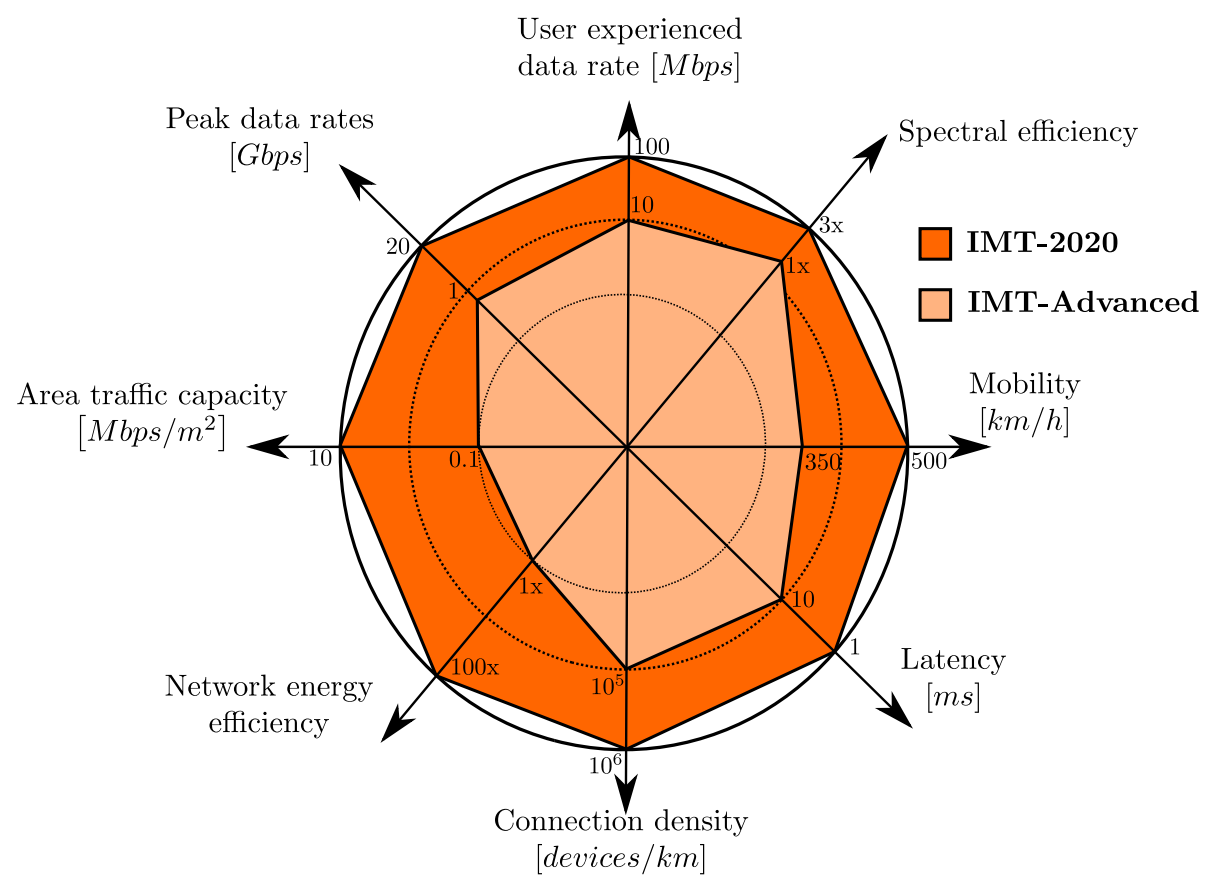

Figure 1.1: Performance improvement from IMT-Advanced to IMT-2020 [3].

of the International Telecommunication Union (ITU) for International Mobile Telecommunication (IMT) mobile systems, also known as IMT-Advanced. These requirements were surely unthinkable a few years ago but now are a reality. To illustrate this, peak data rates of 1 Gbps with a bandwidth of 100 $\mathrm{MHz}$ in downlink, a very low latency, and a more efficient management of the interference are the main examples of why LTE-Advanced is so appealing for operators. In this way, LTE-Advanced users may enjoy data rates similar to those of the optical fiber users. To attain these levels of capacity and quality, both the international scientific community and, in particular, the 3GPP continue developing diverse technological solutions for LTE. In particular, the main features that have been introduced in LTE-Advanced are the support of advanced aggregation of the spectrum, advanced Multiple Input Multiple Output (MIMO) techniques, some enhancements in the operation of Home eNodeBs (HeNBs), and relaying [2].

On the path towards the Fifth Generation $(5 \mathrm{G})$ mobile, the $3 \mathrm{GPP}$ held a workshop in 2015 in which three use cases were identified with high priority: enhanced Mobile BroadBand (eMBB), massive Machine Type Communications 
(mMTC), and Ultra-Reliable and low-latency Communications (URC) [3, 4]. These use cases include among others self-driving car services, health, energy, and manufacturing. Figure 1.1 shows the expected capabilities for the future $5 \mathrm{G}$ and its evolution from IMT-Advanced to IMT-2020. Despite the fact that it is still too early to identify the main features of the new 5G wireless technology, there is a consensus in the fact that there will be a new air interface, not backward compatible with LTE-Advanced. Ericsson recently coined this new air interface as NX.

\subsubsection{G standardization process}

The Working Party (WP) 5D of the ITU, responsible for the radio interface aspects of IMT systems, will lead for the $5 \mathrm{G}$ a process similar to that of the development of IMT-Advanced systems. This process will be formally initiated with the dispatch of a Circular Letter by ITU along 2016. It is worth noting that the LTE evolution will continue in parallel to the specification of the $5 \mathrm{G}$ radio access technologies. Figure 1.2 shows the timeline to be followed by the International Telecommunication Union Radiocommunication (ITU-R) sector and the 3GPP to define this new 5G radio access technology. In 2016 and 2017, the WP 5D will define the performance requirements, evaluation criteria, and assessment methodology of the new IMT radio interface. At the end of 2017 and until mid-2019, different proposals will be sent to the ITU-R sector, including most likely a 3GPP candidate, maybe the only one. Finally, the evaluation of various technologies will be carried out by some external evaluation groups from 2018 to 2020. It is expected that the external proponents will send the final specification of the radio access technologies, at the latest, in February 2020. The process will finalize with a new recommendation for the initial release of International Mobile Telecommunication 2020 (IMT-2020).

Although the requirements of the new $5 \mathrm{G}$ radio access technology have not yet been released, it is expected that the development of the 5G will occur in two phases. In the first phase, the efforts are focused on the integration of the $5 \mathrm{G}$ network with LTE, working at least at frequencies below $6 \mathrm{GHz}$. In a second phase, it is expected to evolve the systems towards an independent operation to LTE with the possibility of using frequencies above $6 \mathrm{GHz}$. In this sense, a new study item within $3 \mathrm{GPP}$ on the channel modeling above $6 \mathrm{GHz}$ has been approved [5]. 


\section{CHAPTER 1. INTRODUCTION}

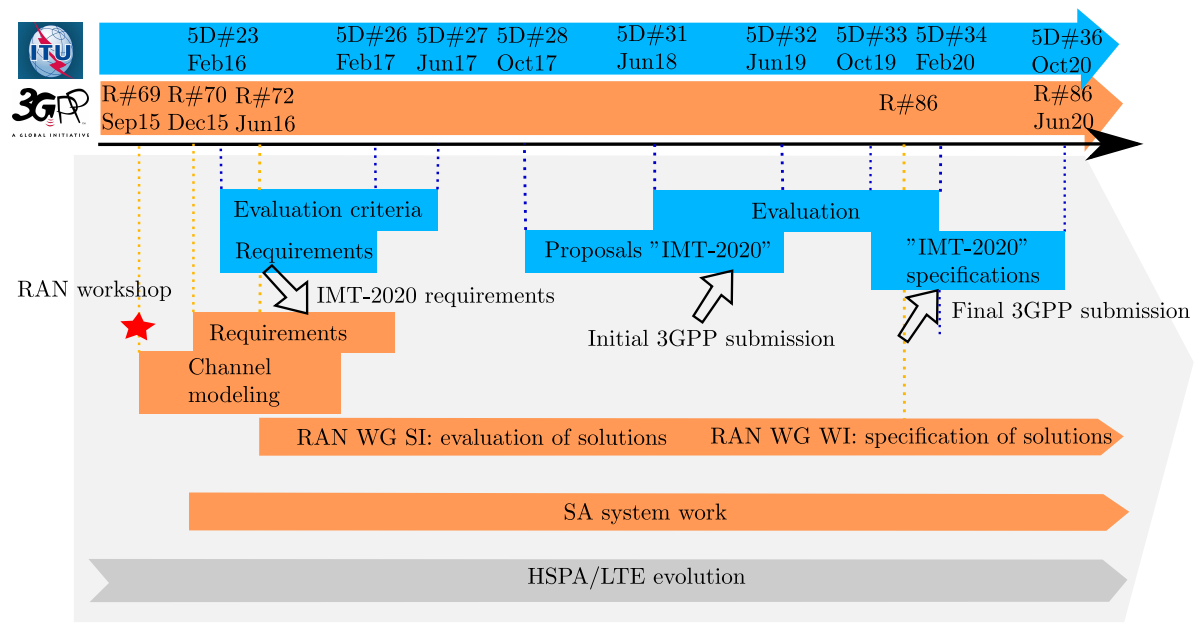

Figure 1.2: Planned timeline for IMT-2000 [4].

\subsection{State of the art analysis}

The cooperative transmission among nodes is foreseen to be one of the pillars of the new $5 \mathrm{G}$, being the system natively designed with this working principle. In this sense, there are several open research aspects related to the use of cooperative transmission schemes in $5 \mathrm{G}$ mobile networks. This section aims at analyzing the current sate of the art concerning this topic, in which this Thesis focuses.

\subsubsection{Relaying}

Relaying is one of the main hot topics for the next-generation wireless standards [6]. This technology is usually used to extend the coverage to remote users, boost offered data rates, and decrease the energy consumption. The simplest relaying scheme works under half-duplex operation, which leads to an inefficient use of resources due to the time multiplexing between the transmissions of the source and the relay at the same frequency. However, this operation mode avoids the self-interference on the reception and transmission of the relay. Figure 1.3 describes the two phases of the transmission. In the first phase, the source transmits information to the relay (and possibly also to the destination), and in the second phase, the relay node forwards the processed data to the destination, remaining silent the source. This transmission type entails 

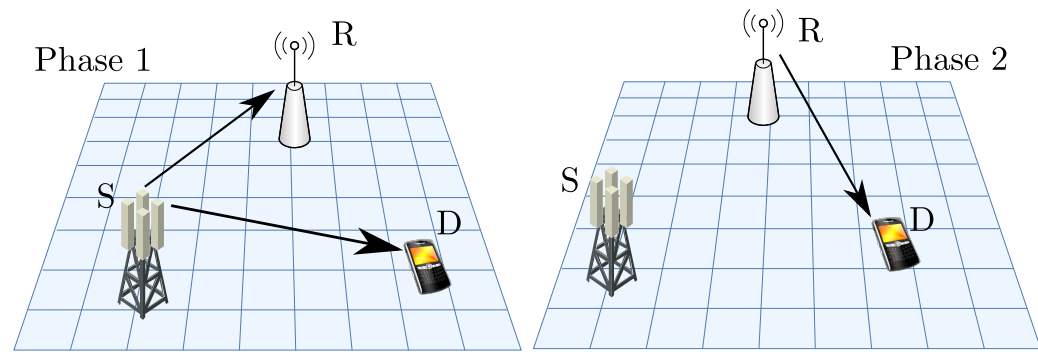

Figure 1.3: Half-duplex operation [6].

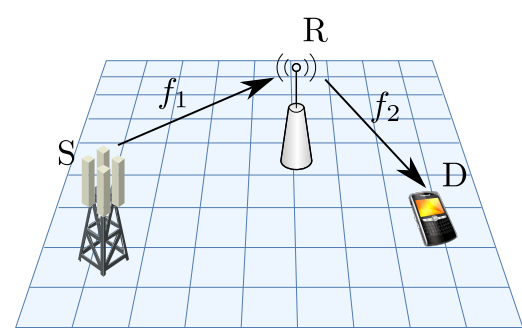

Figure 1.4: Out-band full-duplex operation [6].

that the source can only transmit for half of the time. In the literature, these schemes are known as non-orthogonal schemes [7].

There exist some relays that can transmit and receive at the same time in an out-band full-duplex operation. Figure 1.4 shows the full-duplex operation, where the relay receives and transmits at different frequencies. This scheme suffers from the same spectrum efficiency loss as the half-duplex relay, since additional frequency resources are needed for relay transmissions.

To overcome the inefficiency of the out-band full-duplex schemes, some systems mimic the in-band full-duplex operation in which the relay can transmit and receive simultaneously at the same frequency band. These systems fall into the following four categories [6]:

- Successive relaying is composed of two half-duplex relays as shown in Figure 1.5 [7]. In this scheme, each relay receives new information from the source ( $R_{1}$ in phase 1 and $R_{2}$ in phase 2 ), while the other relay forwards the information received in the previous stage to the destination ( $R_{2}$ in phase 1 and $R_{1}$ in phase 2 ). In this way, the source can transmit a new block at each time slot obtaining the same gain multiplexing than the in-band full-duplex operation. On the other hand, since both relays 

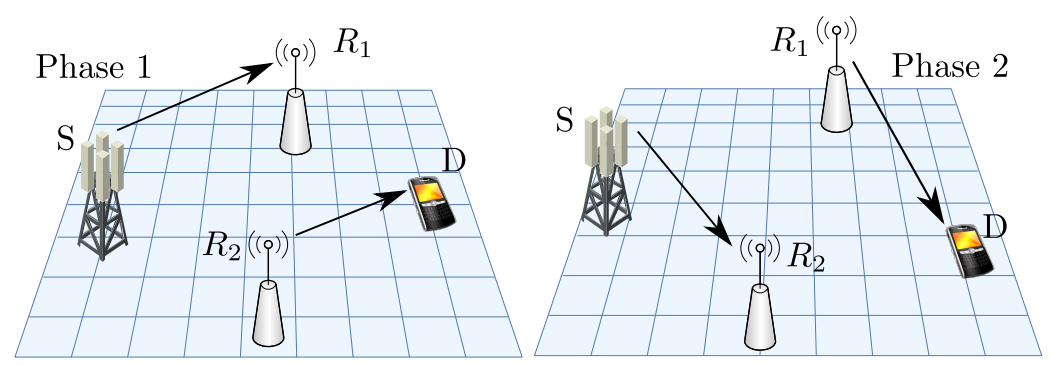

Figure 1.5: Successive relaying [6].
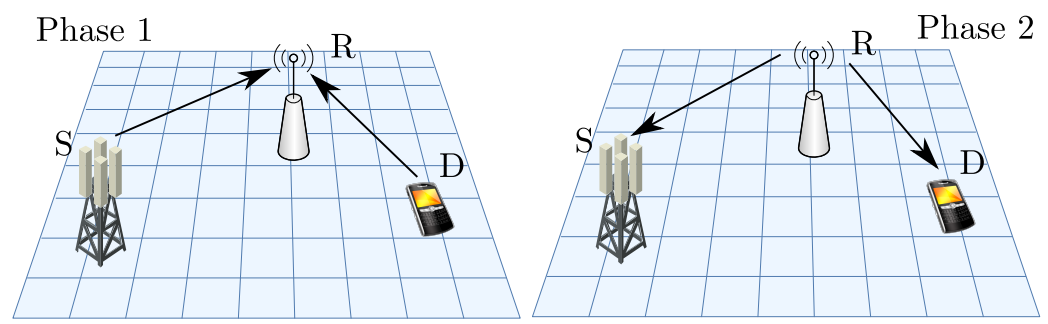

Figure 1.6: Two-way relaying [6].

$R_{1}$ and $R_{2}$ are half-duplex, the relays should be selected to be isolated from each other. Moreover, perfect synchronization is needed between the source and the relays.

- Two-way relaying method consists in the interchange of information between two nodes with the help of a relay [7]. As shown in Figure 1.6, in the first phase, both the source and destination transmit their data simultaneously. Then, the relay broadcasts the superposition of both signals. In this case, both nodes will first subtract its message from the received signal and detect the information from its partner at the end of the second phase. Similarly to successive relaying, perfect synchronization between the source and the destination is required.

- Buffer-aided relaying method chooses when transmitting or receiving based on the quality of the source-relay and relay-destination links. As shown in Figure 1.7, if the quality of the source-relay link is higher than the quality of the relay-destination link, the relay will receive and store the information in buffers. However, if the quality of the relay-destination link is greater than the quality of the source-relay link, the stored infor- 

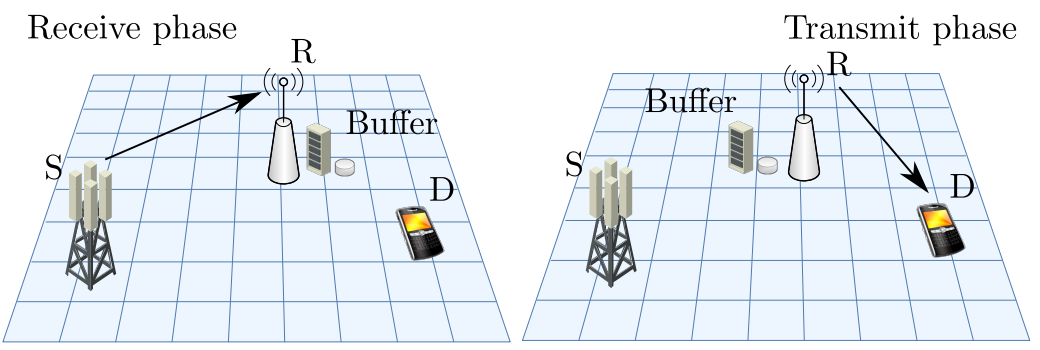

Figure 1.7: Buffer-aided relaying [6].
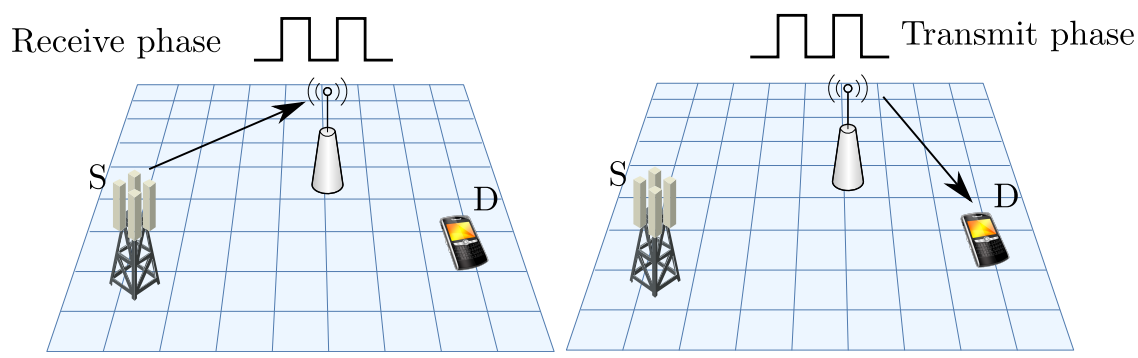

Figure 1.8: Frame-level virtual full-duplex relay [6].

mation will be transmitted to the destination. This scheme outperforms the half-duplex scheme at the expense of an increased packet delay.

- Frame-level virtual full-duplex relay is based on on-off division duplexing, enabling full-duplex communication at the frame level. As shown in Figure 1.8, the relay transmits its signal through on slots of a randomly generated mask and receives information through off slots. In this way, the relay may transmit and receive simultaneously in the same frame at the expense of adding a small queuing delay less than one frame.

To neutralize the spectrum inefficiency of the schemes mentioned above, the research community is focusing its attention on in-band full-duplex systems in which the relay transmit and receive at the same time and the same frequency. To do so, an interference cancelation mechanism or some transmit/receive antenna isolation mechanism are necessary for the relay. Figure 1.9 illustrates the operation diagram of an in-band full-duplex relay. 


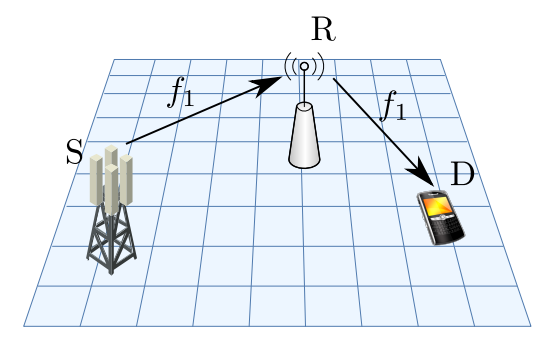

Figure 1.9: In-band full-duplex operation [6].

\subsubsection{D2D communications}

Device-to-Device (D2D) communication underlying cellular networks has attracted much attention in the last years, mainly because the promising gains this communication promises concerning data rates, low delays, low power consumption, reuse gain, and resource savings [8, 9]. Although some attempts have been carried out in unlicensed spectrum operation of Bluetooth or WiFi Direct, there are some doubts about spectrum and services availability. For this reason, the integration of D2D communications has been delayed over the years. However, in LTE-Advanced Release 13, the 3GPP is discussing its feasibility. Some solutions proposed by 3GPP are discussed in Section 2.4. Doppler et al. presented a solution for a D2D session setup using dedicated signaling and automatic handover from cellular traffic to D2D traffic [8]. Fodor et al. showed the key design aspects of D2D communication [9]. The authors analyzed the D2D communication scenarios, peer discovery techniques, Radio Resource Management (RRM) alternatives, channel quality estimation, and power control.

New open issues need to be solved so as to allow a smooth integration of D2D communication into the IMT-Advanced system. For instance, in an Orthogonal Frequency Division Multiplexing (OFDM) system, in which some of the Resource Blocks (RBs) are reused by the D2D User Equipments (UEs) ${ }^{1}$, intra-cell interference is no longer negligible [10-12]. The sharing of these RBs, along with interference avoidance mechanisms, is another hot topic in the literature $[13,14]$. Janis et al. proposed to limit the maximum power of the D2D UEs to avoid interference to cellular UEs based on the cellular uplink power control information [13]. Yu et al. analyzed two RRM schemes based on optimization techniques [14]. Both schemes aim at maximizing the total throughput while satisfying certain constraints. The first scheme is limited in

\footnotetext{
${ }^{1}$ Throughout this Thesis, the terms User Equipment (UE) and Mobile Node (MN) are interchangeable.
} 
the maximum power, and the second one guarantees a minimum transmission rate while prioritizing cellular UEs. Besides, an upper limit to the spectral efficiency is set to consider practical limitations in modulation and coding schemes. With non-orthogonal sharing, the optimal power allocation resides on a finite set of feasible solutions. Such non-orthogonal sharing provides most of the gain. With orthogonal sharing, the authors solved the optimum radio resource allocation in closed form, except for the case when maximum transmit power is limited.

Interference between D2D and cellular networks is today another hot research topic [10]. Peng et al. proposed two mechanisms for interference avoidance from cellular UEs to D2D UEs and vice versa. The first scheme assumes that some uplink RRM information is available at each UE. Thanks to this information, each UE measures the interference caused by another UE and stores this information in such a way that if interference value is higher than a certain value, then the D2D UE reuses other RBs. In the second scheme, the Base Station (BS) broadcasts to the D2D UEs a table with the tolerable interferences. Each UE calculates an estimated interference level and chooses the RBs whose interference level is assumable. Min et al. proposed a limited interference area in which cellular UEs are not allowed to use the same resources as D2D users [11]. This limited area is defined as the area in which the interference to signal ratio is greater than a given threshold. Janis et al. studied novel precoders for cellular downlink transmissions to avoid interference to the D2D terminals [12]. Other references can be found related to interference control $[15,16]$ or RRM $[17,18]$.

\subsection{3 $\mathrm{MIH}$}

The Institute of Electrical and Electronic Engineers (IEEE) 802.21 Media Independent Handover (MIH) standard was specially designed to perform handovers among different IEEE 802 architectures [19]. In [20] Bae et al. proposed to use MIH signaling to support a triggering mechanism for the management of vertical handovers based on the data rate. On the other hand, Seol and Chung proposed to conduct vertical handover between LTE and Worldwide Interoperability for Microwave Access (WiMAX) by adding some new nodes to the core network with a MIH Function entity (MIHF) [21]. The work from Bultmann et al. [22] addressed handover signaling and introduced some procedures for handover in heterogeneous networks assuming LTE assisted with fixed relays. It is worth noting that so far MIH signaling has only been used to make handovers between BSs of different networks rather than to select relays. 


\subsubsection{Coherent space-time schemes}

Since Telatar [23] and Foschini [24] discovered that using multiple antennas at the transmitter and the receiver implies to increase the spectral efficiency significantly, the international scientific community has turned its attention to the development of these systems. Traditionally, MIMO systems have been used in two different ways, to obtain diversity gain and spatial multiplexing gain. The maximization of both gains cannot be jointly achieved, and there is a fundamental trade-off between both gains [25]. In particular, higher transmission rate implies less reliability and more diversity means less transmission rate.

Witnebben was the first one to develop a bandwidth efficient transmit diversity scheme that consists in sending several replicas of the same information from several transmit antennas but with a delay of a symbol interval [26]. Later, Foschini proposed a scheme to improve the transmission rate with the Bell labs LAyered Space-Time (BLAST) architectures [24]. Tarokh et al. proposed a new class of codes referred to as Space-Time Codes (STC) that outperforms the performance of rate- $1 / 2$ repetition codes of Witnebben [27]. There are two types of STC, namely Space-Time Block Codes (STBCs) and trellis codes, which provide full diversity and coding gain, respectively. The most famous STBC is the Alamouti code, which presents a remarkable simple detection through linear processing with maximum likelihood [28]. This code belongs to the category of Orthogonal Space-Time Block Codes (OSTBCs) for two transmit antennas. Tarokh et al. generalized this result to an arbitrary number of transmit antennas using complex orthogonal matrices and maintaining the performance of Maximum Likelihood (ML) decoding with linear processing [29]. These schemes provide full diversity at the expense of reducing the achievable rate. Hence, the recent works on non-orthogonal schemes such as the QuasiOrthogonal Space-Time Block Codes (QOSTBCs), which relax the requirement of linear processing at the receiver, to obtain high data rates.

\subsubsection{Non-coherent space-time schemes}

Coherent communications dominate the current cellular technologies such as LTE-Advanced, WiMAX or High Speed Packet Access (HSPA)+. These systems require a complete a priori Channel State Information (CSI) at least at the receiver side. This information is obtained with pilot-based estimation techniques in which resources are reserved for pilot signaling to estimate the channel characteristics.

How much training is needed in MIMO links? This question was formulated by Hassibi and Hochwald in [30]. They concluded that if pilots and data 
power are allowed to vary, the optimal number of pilots is equal to the number of transmit antennas. On the contrary, if power is required to be equal, the optimal number of symbols could be larger than the number of transmit antennas. If channel coherence time tends to infinity or when the Signal to Noise Ratio (SNR) is high, the penalty of data rates due to pilots is neglected. Otherwise, training-based schemes are highly suboptimal.

All these problems above-mentioned could be at least partially solved through non-coherent communication in which neither the transmitter nor the receiver has any a priori knowledge of the channel realization. Marzetta and Hochwald obtained that the non-coherent ergodic capacity is achieved when the transmitted signal matrix is composed of the product of two statistically independent matrices: a $T \times T$ isotropically distributed unitary matrix and a certain $T \times M$ random matrix that is diagonal, real, and non-negative [31] [32], where $M$ is the number of transmit antennas, and $T$ is the channel coherence time. This modulation type is called Unitary Space-Time Modulation (USTM). Moreover, they concluded that for a fixed number of transmit antennas, as $T$ increases the capacity approaches the capacity obtained as if the receiver knew the propagation coefficients. In the same way, when SNR is high, non-coherent capacity approaches coherent capacity. On the other hand, for any $T$, any number of receive antennas $(N)$ and any number SNR, capacity for $M>T$ is equal to the capacity of $M=T$.

Zheng and Tse investigated the ergodic capacity in the high SNR regime [33]. The authors concluded that the identity matrix can substitute the random diagonal matrix without any capacity penalty. Therefore, the optimal signal structure is a matrix whose columns are isotropically distributed orthogonal vectors. Moreover, based on geometric insights concluded that at high SNR, the subspace of the transmitted signal matrix is maintained in the received signal matrix in a Rayleigh block-fading channel. Therefore, information of the transmitted signal is contained in the subspace of the received signal, and the particular orientation of the received signal vectors within the subspace lacks information [34]. The complex channel simply rotates and scales the bases of this subspace. These observations suggest that, for the non-coherent channel, at high SNR, the optimal design of constellations must be focused on the bases of a set of linear signal subspaces rather than the design of the actual signal values. Natural geometric objects of relevance to the design of constellations are based on the Stiefel and Grassmannian manifolds [33].

In the low SNR regime, coherent and non-coherent capacities are asymptotically equal [33]. Only an increase of the receive antennas can enhance the capacity because of collecting more power of the information signal. The optimal approach is to allocate all the transmit power to only one antenna during one symbol period. 


\section{CHAPTER 1. INTRODUCTION}

There exists another type of non-coherent communication called Differential Unitary Space-Time Modulation (DUSTM) for multiple antennas [35]. It can be seen as a natural extension of Differential Phase Shift Keying (DPSK) commonly used in single-antenna systems. For multiple antennas, each transmitted signal matrix is coded taking into consideration the previously transmitted signal matrix, which both matrices are unitary. The detection is, in the same way, differential. Because of detection uses the received signal in two instants, a 3 $\mathrm{dB}$ performance loss is obtained respect to a coherent system. To simplify the design of unitary matrices, Hassibi and Hochwald proposed a class of Cayley codes that works for any number of antennas [36].

As before mentioned, USTM is capacity-achieving, but it often incurs exponential demodulation complexity in $R$ and $T$ according to the constellation size. For example, if the transmission rate is eight bits per channel use and the channel coherence time is four channel uses, then the number of matrices of the constellation is $2^{8 \cdot 4} \approx 4 \cdot 10^{9}$. On the other hand, training-based schemes simplify the receiver design, but they induce certain SNR loss due to imperfect CSI. To achieve desirable trade-offs between complexity and performance, Yu and Giannakis proposed a training approach where USTM symbols over a short length $T_{\tau}(<T)$ were used as pilots, which carry information to the receiver, and coherent communication is used for the remainder of the block [37]. This scheme presents three main advantages. First, unlike traditional training-based schemes, here the non-coherent symbols that are used to estimate the channel can carry information. Second, the length $T_{\tau}$ is a small fraction of $T$, leading to low decoding complexity. Finally, the trade-off between complexity and performance can be controlled through $T_{\tau}$.

\subsection{Problem formulation}

Under optimal radio conditions, it is expected that relays will not only offer enhancements related to network coverage but also, it will improve the data rates of users with good radio conditions. These envisioned gains are detected in systems with a fixed number of static relays. However, slight deviations of these assumptions can result in a significant degradation of achievable data rates and coverage. Consequently, the obtained gains for fixed relaying cannot be extrapolated to mobile relaying.

In $3 \mathrm{GPP}$, the mobile relay concept has been traditionally identified with a fixed node located at the top of a vehicle that provides service to the users located within the vehicle. The high mobility of the vehicle poses a bottleneck in the performance of the base station-relay link. It is expected that the channel estimation with the current pilot allocation suffers significant degradation 
unless the percentage of pilots is increased. This fact would cause a reduction in the resources assigned for data, and thus, in the user throughput as well. This problem could be aggravated when increasing the number of transmit antennas. As an alternative to this scenario, in this Thesis, a UE acting as a relay is also considered as a Mobile Relay (MR). Throughout this Thesis, the first scenario will be referred to as moving network.

In contrast to fixed relaying proposed in LTE-Advanced, a MR is typically constrained in processing and battery. The retransmission of information entails to the user a waste of energy that should be rewarded in some way. Furthermore, the location cannot be controlled, and it is far away being uniform throughout the scenario. Nonetheless, in point-to-point communication systems, the use of multiple antennas has received much attention in the literature due to its ability to improve data throughput and spectral efficiency. The same ability is foreseen in a mobile relaying scenario. However, these envisioned gains are subjected to the perfect knowledge of the CSI. In practical systems, this assumption is no longer fulfilled, and some degradation is suffered, especially in high-speed environments. In these scenarios, it is envisioned that the channels between the source and the relay, the relay and the destination, and the source and the destination could undergo rapid variations, decreasing the benefits of MIMO. Moreover, performing coherent detection in relay networks consumes more resources than in point-to-point communications. To overcome these issues, the source and the relay could use a signaling scheme that does not require CSI knowledge in the data detection. This type of communication is typically referred to as being non-coherent.

\subsection{Objectives and Thesis scope}

This Thesis aims at investigating the integration of mobile relays into $5 \mathrm{G}$ mobile broadband networks, paying particular attention to the selection problem and the use of non-coherent signaling. The main goal is to evaluate and propose signaling, medium access, and networking techniques for mobile relaying. This main objective can be divided into two partial objectives listed below

- Network-layer. To design and assess an upper layer signaling framework that manages mobile relays, including

- Integration of MIH signaling to manage multiple radio interfaces in a cellular system assisted by MRs.

- Development of a relay selection mechanism through MIH signaling.

- Simplified financial analysis of the inclusion of mobile relaying into LTE-Advanced systems. 


\section{CHAPTER 1. INTRODUCTION}

- Development of a simulation platform that allows for the assessment of the performance of the designed schemes.

- Physical-layer. To assess the use of non-coherent techniques for a blind transmission in the mobile relay scenario, including

- Performance comparison between non-coherent techniques and state-of-art training-based coherent schemes over time and frequency varying spatially correlated channels.

- Suboptimal detection in multi-user scenarios.

- Behavior under realistic channel conditions in an LTE system.

- Integration of Grassmannian signaling for the future 5G cellular systems.

- Development of a simulation platform suited for the performance evaluation of such non-coherent techniques.

\subsection{Research methodology}

Given the enormous complexity of current and future wireless systems such as IMT-2020 systems, it is not practical to evaluate their performance using analytical methods. For this reason, system modeling and computer simulation represent a real alternative for the assessment of these systems, achieving a good trade-off between complexity, cost, time of development and accuracy. A possible focus for the simulation of a wireless system would be to consider a global network model that emulated the interaction between many BSs and UEs. This simulation would take traffic aspects into account together with the users' mobility while reproducing, at the same time, channel coding, modulation, and propagation aspects associated to each one of the wireless links. However, such a complete simulation model would suppose a prohibitive computational burden. To reduce such complexity, simulation is usually divided into two stages

- Link-level simulations are used to assess the performance of the physical layer plus those Medium Access Control (MAC) aspects directly related to the radio interface, such as the Hybrid Automatic RepeatreQuest (HARQ) mechanisms. At link level, a continuous radio link is modeled, including in the simulation specific features such as synchronization, modulation, channel coding, channel fading, channel estimation, and demodulation. The main results of these simulations allow characterizing the user Quality of Service (QoS), measured in terms of either Bit Error 
Rate (BER) or BLock Error Rate (BLER), as a function of the Signal to Interference plus Noise Ratio (SINR).

- System-level simulations allow evaluating the performance of the global network. At this level, system modeling comprises a set of BSs and all their associated user terminals. The signal level received by each user, as well as other users' interferences, is modeled taking into account the propagation losses and channel fading effects. Besides, in dynamic system-level simulators, users' mobility and traffic generation are also included together with all RRM operations, such as link adaptation, dynamic resource allocation, and handovers. SINR is calculated for each active user taking into account the current configuration of the network. These SINR values can be then translated to BER or BLER values using the results obtained in the link-level simulations. The interaction between link and system-level simulators is usually referred to as Link to System (L2S) interface. Note that, as aforementioned, both levels of the simulation are evaluated independently.

This Thesis uses both levels of simulations to evaluate the different schemes designed along the dissertation. Thanks to the utilization of these simplified analysis tools, proper conclusions are made, highlighting design criteria and optimum configurations.

\subsection{Thesis outline and main contribution}

This Thesis is organized into five chapters and five appendices as follows:

- Chapter 2 shows an exhaustive review of the main features of the $5 \mathrm{G}$ cooperative technologies that are under ongoing study in the 3GPP and the Mobile and wireless communications Enablers for Twenty-twenty (2020) Information Society (METIS) project. In particular, the review covers fixed, moving networks, D2D, and Vehicle to Everything (V2X) communications.

- Chapter 3 proposes a new application of MIH signaling to the management of multiple radio interfaces in a cellular system assisted by MRs.

- Chapter 4 assesses the performance of non-coherent transmission schemes compared to training-based schemes and the integration of Grassmannian signaling in the future $5 \mathrm{G}$ cellular networks.

- Chapter 5 outlines the main conclusions of this Thesis and presents the future work of this research. 
- Appendix A describes the link-level simulator used in this dissertation to obtain all performance results. Moreover, this section presents some features of the system-level simulator such as the path loss equation of a Wireless Local Area Network (WLAN) IEEE 802.11n link, the SINR distributions of the IMT-Advanced scenarios, and the look-up tables of the Wireless Fidelity (WiFi) and LTE systems.

- Appendix B presents some basics on USTM, and in particular, on Grassmannian signaling.

- Appendix C calculates the SNR of the system model used throughout this Thesis.

- Appendix D calculates some of the equations of Section 4.6.

The main contributions of this Thesis are summarized in the following subsections, including the references to the articles derived from this dissertation. After the summary of contributions, a complete list of publications can be found in the next section.

\subsubsection{Chapter 2 - Features and challenges of the cooper- ative $5 \mathrm{G}$ schemes}

This chapter shows an exhaustive review of the key cooperative techniques that are under study in 3GPP and other research projects such as METIS [38]. Some of these techniques will be enhanced and updated within the $5 \mathrm{G}$ network.

The chapter describes the main functionalities of the fixed relay as well as the different categories of these nodes. Next, the main drawbacks of the moving networks that give service to users located in high-speed vehicles are identified. Concerning D2D communications, a brief tutorial on the fundamentals of 3GPP Proximity-based Services (ProSe) is provided, including scenarios, evaluation methodology, metrics, channel modeling, and radio aspects such as synchronization, discovery, and communication. This communication among devices is introduced in vehicular environments with the critical objective of avoiding accidents. The chapter outlines the initial evaluation designs of V2X services.

\subsubsection{Chapter 3 - Network-layer solution: MIH for mo- bile relaying}

The main contribution of this chapter is the proposal of a new application of MIH signaling to the management of multiple radio interfaces in a cellular sys- 
tem using MRs, known as Media Independent Handover-Driven Relay Selection mechanism (MIDRES).

The chapter analyzes three different scenarios wherein the MIDRES algorithm is executed if performance is inferior to a given threshold. For each scenario, the different phases of the routing and handover procedure for mobile relaying are analyzed. Once the routing mechanism is presented, an optimum threshold is obtained after showing a trade-off between channel quality improvement and signaling overhead. The optimization is based on the minimization of the number of relays and, secondly, maximizing the Cell-Edge User Throughput (CEUT). For this trade-off, an optimum number of relays is also identified for indoor and urban microcell scenarios. The chapter also describes a technique that reduces the signaling overhead by restricting the search area. The effect of the velocity on the performance of MIDRES mechanism is also analyzed. Finally, a simplified financial analysis of the inclusion of MRs in the LTE-Advanced architecture is discussed.

\subsubsection{Chapter 4 - Radio solution: non-coherent commu- nications schemes}

This chapter investigates the use of non-coherent communication techniques for open-loop transmission as a solution to the drawbacks of current coherent systems. The chapter starts describing these drawbacks and their partial solutions proposed by $3 \mathrm{GPP}$. Next, five main contributions are identified:

- Contribution 1. A performance comparison between several state-ofart training-based coherent schemes are compared with two non-coherent schemes under realistic channel conditions for two and four antennas. In particular, the non-coherent MIMO communication schemes are DUSTM and Grassmannian signaling. The selected coherent baselines for two transmit antennas are the Alamouti and Golden codes and, for four transmit antennas, the rate- $1 / 2$ OSTBC and the Quasi-OSTBC. The study focuses on frequency-flat temporally-correlated Rayleigh-fading MIMO channels. The measured performance is the Frame Error Rate (FER) against the normalized Doppler frequency.

- Contribution 2. Grassmannian schemes achieve the ergodic high SNR non-coherent MIMO capacity for standard single-channel block-fading communications scenarios. To enable the application of the Grassmannian signaling in multi-channel-frequency-flat systems, two techniques that provide an equivalent single-channel block-fading system are proposed. The first technique consists in reshaping the transmitted pulse to 
the coherence bandwidth. The second technique consists in concatenating the subchannels within the coherence bandwidth. Finally, a numerical comparison is proposed between the spectral efficiency of concatenated and independent single-channel Grassmannian signaling schemes when utilized in systems with more than one subchannel.

- Contribution 3. A performance comparison between the coherent transmit diversity modes of LTE are compared with Grassmannian signaling over time and frequency spatially correlated channels under the same physical configuration as LTE technology. The coherent schemes are the Space-Frequency Block Coding (SFBC) and Frequency-Switched Transmit Diversity (FSTD) for two and four transmit antennas, respectively. Note that this study also takes into consideration in its simulations the multi-carrier nature of the OFDM-based systems. Moreover, the Chapter discusses the appropriate resource mapping of Grassmannian signaling in vehicular scenarios.

- Contribution 4. This Chapter extends the use of single-user noncoherent communications seen so far to multi-user MIMO framework by combining superposition coding with a reduced-complexity detection method. Numerical results confirm that this scheme achieves higher user rates than non-coherent Multi-User (MU) transmission based on time multiplexing. In addition to the well-known sum-rate gain of MU systems, an extra performance gain given by downlink non-coherent MU communication is shown and qualitatively justified.

- Contribution 5. This chapter discusses the integration of Grassmannian signaling for multi-hop communications, D2D communications and moving networks. This chapter proposes the use of non-coherent carriers wherein Grassmannian signaling could be transmitted. Finally, a multipoint coordinated transmission scenario is considered to study the power imbalance among Transmission Points (TPs) for the Alamouti, Golden, and Grassmannian schemes. 


\subsection{List of publications}

The work developed during this Thesis made possible the publication of the following journals, conference papers, reports with special relevance and book chapters.

\section{International journals}

[J1] David Martín-Sacristán, José F. Monserrat, Jorge Cabrejas Peñuelas, Daniel Calabuig, Salvador Garrigas, and Narcís Cardona, "On the way towards fourth-generation mobile: 3GPP LTE and LTE-Advanced," EURASIP Journal on Wireless Communications and Networking, vol. 2009, pp. 1-10, March 2009.

[J2] David Martín-Sacristán, José F. Monserrat, Jorge Cabrejas Peñuelas, Daniel Calabuig, Salvador Garrigas, and Narcís Cardona, "3GPP Long Term Evolution: Paving the Way towards Next 4G," Waves, vol. 1, 2009.

[J3] David Martín-Sacristán, José F. Monserrat, Vicente Osa, and Jorge Cabrejas, "LTE-Advanced System Level Simulation Platform for IMTAdvanced Evaluation," Waves, vol. 2011, pp. 15-24, 2011.

[J4] Jorge Cabrejas, Pablo Gualda, José F. Monserrat, and David MartínSacristán, "Application of MIH for the lightweight deployment of LTEadvanced systems through mobile relaying," EURASIP Journal on Wireless Communications and Networking, vol. 2012, March 2012.

[J5] Jorge Cabrejas, Pedro Chaparro, José F. Monserrat, and Narcís Cardona, "Reaching Network Ubiquity through a New Concept of Mobile Terminal," Waves, no. 4, pp 89-97, 2012.

[J6] Sandra Roger, Daniel Calabuig, Jorge Cabrejas, and José F. Monserrat, "Multi-User Non-Coherent Detection for Downlink MIMO Communication," IEEE Signal Processing Letters, vol. 21, no. 10, pp. 1225-1229, October 2014.

[J7] Sandra Roger, Jorge Cabrejas, Daniel Calabuig, José F. Monserrat, Yaser M. M. Fouad, Ramy H. Gohary, and Halim Yanikomeroglu, "Noncoherent MIMO Communication for the 5th Generation Mobile: Overview and Practical Aspects," Waves, no. 6, pp 5-13, 2014.

[J8] Yaser M.M. Fouad, Ramy H. Gohary, Jorge Cabrejas, Halim Yanikomeroglu, Daniel Calabuig, Sandra Roger, and José F. Monserrat, "TimeFrequency Grassmannian Signalling for MIMO Multi-Channel-Frequen- 
cy-Flat Systems," IEEE Communications Letters, vol.19, no.3, pp. 475478, March 2015.

[J9] José F. Monserrat, Irene Alepuz, Jorge Cabrejas, Vicente Osa, Javier López, Roberto García, María José Domenech, and Vicent Soler, "Towards User-Centric Operation in 5G Networks," EURASIP Journal on Wireless Communications and Networking, vol. 2016, no. 1, pp 1-7, $2016 .^{23}$

\section{International conferences}

[C1] David Martín-Sacristán, Jorge Cabrejas, Daniel Calabuig, and José F. Monserrat, "MAC Layer Performance of Different Channel Estimation Techniques in UTRAN LTE Downlink," in 69th IEEE Vehicular Technology Conference (VTC Spring 2009), pp.1-5, April 2009.

[C2] José F. Monserrat, Pawel Sroka, Gunther Auer, Jorge Cabrejas, David Martín-Sacristán, Albena D. Mihovska, Roberto Rossi, Andreas Saul, and Rainer Schoenen, "Advanced Radio Resource Management for IMTAdvanced in WINNER+ (II)," in Future Network and Mobile Summit, pp. 1-9, June 2010 .

[C3] Berend W.M. Kuipers, Susana Sargento, José F. Monserrat, Jorge Cabrejas, Pedro Chaparro, Hugo D. C. Pires, João M. Soares, Pedro M. Neves, and Mario S. Nunes, "Co-Simulation of ns-2 and SPHERE for Media Independent Handovers," in 5th International ICST Conference on Simulation Tools and Techniques, pp. 139-147, March 2012.

\section{Book chapters}

[B1] Jorge Cabrejas, "El futuro de LTE: LTE-Advanced," in 3GPP LTE: Hacia la $4 G$ móvil, Narcís Cardona, Juan J. Olmos, Mario García, and José F. Monserrat, Marcombo S.A., 2011.

[B2] José F. Monserrat, Jorge Cabrejas, Xavier Gelabert, and Miia Mustonen, "Carrier Aggregation," in Mobile and Wireless Communications for IMT-Advanced and Beyond, Afif Osseiran, José F. Monserrat, and Werner Mohr, Wiley, August 2011.

\footnotetext{
${ }^{2}$ This article is out of the objective of this dissertation.

${ }^{3}$ This paper was also presented in the European cooperation network for scientific and technical research COST IC1004, 2015, http://www.ic1004.org/
} 
[B3] Narcís Cardona, José F. Monserrat, and Jorge Cabrejas, "Enabling Technologies for 3GPP LTE-Advanced Networks," in LTE-Advanced and Next Generation Wireless Networks: Channel Modelling and Propagation, Guillaume de la Roche, Andrés Alayón-Glazunov, and Ben Allen, Wiley, November 2012.

\section{Reports with special relevance}

[R1] WINNER+, "Final evaluation report from Winner+ on the IMT-Advanced proposal in Documents IMT-ADV/6, IMT-ADV/8, and IMT-ADV/9," Doc. IMTADV/ 22, June 2010.

\section{Submitted journal papers}

[S1] Jorge Cabrejas, Sandra Roger, Daniel Calabuig, Yaser M. M. Fouad, Ramy H. Gohary, José F. Monserrat, and Halim Yanikomeroglu, "NonCoherent Open-loop MIMO Communications Over Temporally-Correlated Channels," IEEE Access.

[S2] Jorge Cabrejas, David Martín-Sacristán, Daniel Calabuig, Sandra Roger, and José F. Monserrat, "Integration of Grassmannian Constellations into LTE Networks," IEEE Communications Letters.

\section{Patent under review}

[P1] Jorge Cabrejas, Daniel Calabuig, José F. Monserrat, Sandra Roger, David Gonzálvez, and Peter Fertl, "Method and apparatus for data transmission in non-coherent multi-user MIMO wireless communications systems". 


\section{Chapter 2}

\section{Features and challenges of the cooperative $5 \mathrm{G}$ schemes}

\subsection{Introduction}

Today's users are increasingly demanding higher data rate applications anywhere and anytime. In this situation, data rates offered by mobile operators must be similar to or even higher than traditional ISP. With the purpose of providing service to a large market and as a response to the IMT-2020 process recently triggered by the ITU-R sector, the 3GPP has initiated a process to identify the requirements of a new radio technology for IMT-2020. Without making assumptions about which releases will include the specification of the future 5G, 3GPP keeps working on the main LTE-Advanced enhancements. At the same time, research projects such as METIS have determined a set of five horizontal topics for the $5 \mathrm{G}$ system [39]. In particular, METIS foresees a multi-Radio Access Technology (RAT) that integrates the following schemes: D2D communications, machine-to-machine communications, moving networks, ultra-dense networks, and ultra-reliable communications. These and other technologies are under discussion within the 3GPP to be included in its future standards.

To date, the 3GPP has specified the operation of the fixed relay in Release 10 and 11 [40]. Moving networks are becoming the evolution of fixed relaying for high-speed vehicles, such as trains. However, this study was not completed in the 3GPP due to time constraints, but it is expected to be taken up in future releases. Moreover, with the emergence of social networks, a new paradigm of communication has happened wherein different devices that share a social 


\section{CHAPTER 2. FEATURES AND CHALLENGES OF THE COOPERATIVE 5G SCHEMES}

link can interchange files or messages without having to send that information through the BS. In Release 12 and 13, it is expected to include ProSe among devices to support discovery and direct communication services. As a natural extension of D2D for vehicular environments, 3GPP proposes vehicles' cooperation and also vehicle and infrastructure cooperation so as to improve road safety, and thus avoid accidents.

In this chapter, we review some of the $5 \mathrm{G}$ technologies mentioned above that are being studied in 3GPP and METIS. With this aim, this chapter is divided into the following sections:

- Section 2.2 identifies the different types of fixed relays specified in the 3GPP standards as well as its functionality.

- Section 2.3 outlines the main characteristics of moving networks.

- Section 2.4 details the scenarios, evaluation methodology, metrics, channel modeling and physical layer aspects of D2D communications.

- Section 2.5 details the traditional vehicular protocols and standardization organisms, types, use cases and general design of V2X communications.

- Section 2.6 highlights the most important research areas on mobile relaying and discusses the motivation for the research on non-coherent techniques.

\subsection{Fixed relay}

Cell-edge users often undergo performance degradation that increases with the distance to the BS. To tackle this problem, the $3 \mathrm{GPP}$ proposed the usage of Relay Nodes (RNs) retransmitting information to the destination through the LTE air interface [41]. Cooperative relaying takes advantage of the broadcast nature of the air interface by allowing fixed relays that overhear the transmitted signal so as to make additional transmissions. Such cooperative relaying provides additional diversity against link outages, such as fast fading or shadowing effects. Figure 2.1 shows the LTE-Advanced general architecture including fixed relays. The relay is wirelessly connected to a Donor evolved Node B $(\mathrm{DeNB})^{1}$ through the Un Interface (backhaul link) and connected to UEs through the Uu interface (access link). If the access link shares the same carrier frequency as the backhaul link, this is an in-band relay, being otherwise an out-band relay. In-band relaying entails higher radio resource management

\footnotetext{
${ }^{1}$ Throughout this Thesis, the term evolved Node B (eNB) and Base Station (BS) is interchangeable in an LTE network.
} 
problems for the system as compared to out-band relaying. Moreover, two types of relays have been considered in Release 10 by taking transparency into consideration:

- Non-transparent relay controls the cell the same way as an eNB, that is to say, it transmits its synchronization channels, reference symbols, scheduling information, and HARQ feedback. It also has its Physical Cell Identifier (PCI) that is independent of the donor cell. This nontransparent relay is often used for coverage extension or group mobility, e.g., high-speed vehicles. There are three types of non-transparent relays.

- Type 1 (in-band and half-duplex). The relay is regarded as an in-band relay, and there is a time division multiplexing between backhaul and access link.

- Type 1a (out-band). Different frequency carriers are used for backhaul and access link, i.e. out-band relaying.

- Type $\mathbf{1 b}$ (in-band and full-duplex). The backhaul and access link can operate simultaneously on the same band due to adequate antenna isolation.

- Transparent relay. In this case the UE does not even realize that there is a relay, that is, this node only forwards the information received from the eNB with functionalities of up to layer 2. The eNB directly controls the UEs in the cell and performs radio resource management. Thus, the relay neither transmits synchronization signals, Common Reference Signals (CRSs) nor Physical Downlink Control CHannel (PDCCH). Therefore, this node is only used to enhance the UEs QoS, especially for cell-edge users. This relay is also known as relay Type 2 .

To allow in-band relaying without interferences, some mechanism must be used to share resources between the access link and the backhaul link in the Downlink (DL). As in Type 1b, a secure mechanism is to isolate the transmitter and the receiver so that signals do not interfere themselves. However, this is not always possible. The $3 \mathrm{GPP}$ proposes that the $\mathrm{RN}$ can only receive data from the DeNB in the so-called Multicast-Broadcast Single-Frequency Network (MBSFN) subframes not being able to transmit to the UEs at the same time, in DL. Figure 2.2 illustrates an example of transmission in a scenario with RNs. The arrows show the transmission direction of the radio links. In the first slot, the DeNB transmits information to the UE1 on the access link as usual. In the second slot, the DeNB transmits new information to the RN through MBSFN subframes. Finally, in the third slot, the RN transmits the information previously transmitted by the DeNB to the UE1. Note that in this 


\section{CHAPTER 2. FEATURES AND CHALLENGES OF THE COOPERATIVE 5G SCHEMES}

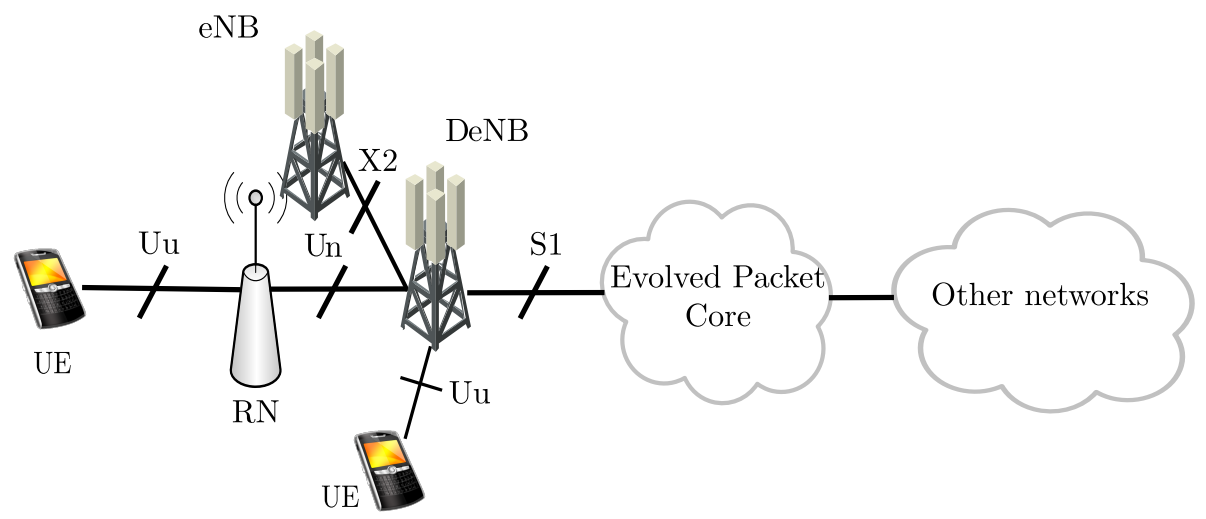

Figure 2.1: General architecture of the LTE-Advanced system with relays.

case the link between source and destination in Figure 1.3 is not contemplated in the $3 \mathrm{GPP}$.

In a scenario with relays Type 1 , the RN has to transmit the PDCCH to the UE even in MBSFN subframes since each UE expects to receive this information in every subframe. For this reason, the relay receives transmissions from the DeNB in MBSFN subframes for a shorter time than normal subframes. Moreover, due to the half-duplex functionality, while the relay is transmitting the $\mathrm{PDCCH}$ to the UE, the relay cannot receive control signaling from the DeNB. Thus, a new control channel called Relay-Physical Downlink Control CHannel (R-PDCCH) located in the Physical Downlink Shared CHannel (PDSCH) area is needed in the backhaul link. This additional signaling overhead reduces the amount of resources available for data information. The R-PDCCH is sent in the fourth OFDM symbol of the first slot for DL assignments and in the last OFDM symbol in the second slot for uplink grants.

Concerning the uplink, the isolation of the access and backhaul link is straightforward since the RN sends the uplink grant scheduling to the UEs and, therefore, has full control over the transmission and reception of UE. On the other hand, the transmission resources of the Physical Uplink Control CHannel (PUCCH) sent by the RN are previously determined by higher layer signaling.

\subsection{Moving networks}

The standardization activity of the 3GPP concerning moving networks started by the end of December 2011, but it was stopped in September 2013 due to time 


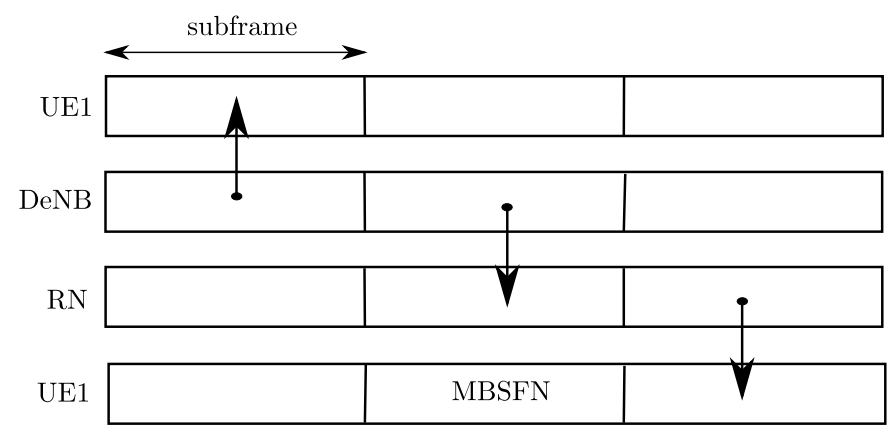

Figure 2.2: Transmission of MBSFN subframes.

constraints [42]. As depicted in Figure 2.3, the scenario under study consists of a relay node situated on a high-speed train with the following features:

- Train velocity: $350 \mathrm{~km} / \mathrm{h}$.

- Known trajectory.

- High penetration losses of the radio signal through the shield carriages.

- UEs on the trains are stationary or move at pedestrian speed.

Some open issues arise when addressing this scenario due to the high speed of the vehicle. First, this speed causes a severe Doppler effect and high penetration losses. Second, HandOver (HO) failure rate may significantly increase hence degrading the received signal and increasing the battery power consumption of UEs. For instance, in a rural scenario in where the cell radius is around

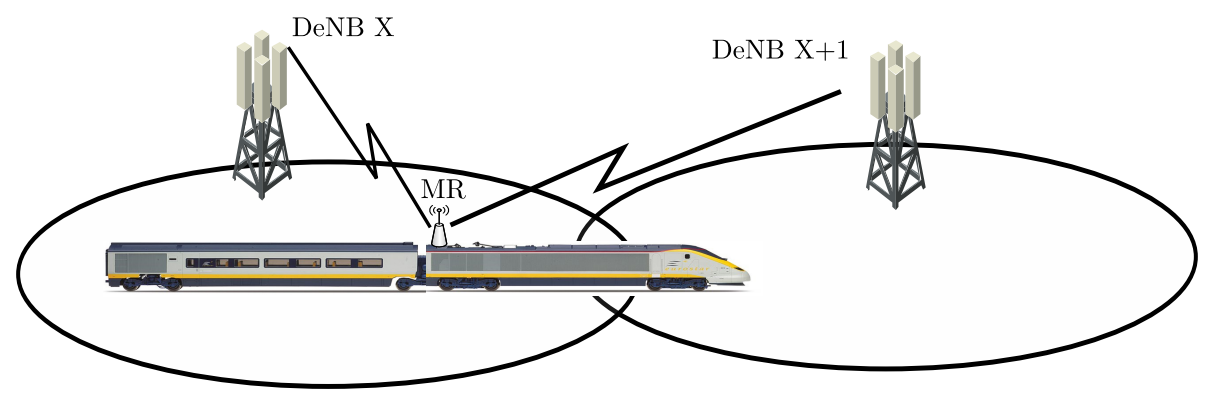

Figure 2.3: Scenario under study for moving networks based on public transportation. 


\section{CHAPTER 2. FEATURES AND CHALLENGES OF THE COOPERATIVE 5G SCHEMES}

$577 \mathrm{~m}$, the train speed provokes a $\mathrm{HO}$ event each 6 seconds. Therefore, it is of paramount importance the management of this type of fast HOs.

To solve these problems and improve the coverage within the train, one or several MRs can be mounted on the high-speed train. These devices provide a wireless backhaul connection via a DeNB along the railway by an outer antenna and also wireless connectivity to the UE inside coaches by internal antennas. Besides, MR can have group mobility capabilities, so that the MR performs a group mobility HO procedure instead of individual HOs. Moreover, thanks to relaying, the transmit power is significantly reduced leading to significant power savings.

\subsection{D2D communications}

The use of smartphones and tablets has favored the emergence of various applications that require a high bandwidth such as gaming, social networks, and content sharing. From a technical point of view, it seems to be beneficial to transmit the information of these applications directly device to device since it allows enjoying a high-rate power-efficient link, with also low latency. Traditional D2D technologies such as Bluetooth and WiFi-Direct work under asynchronous protocols without any infrastructure support. For this reason, users need to be constantly awake to be able to receive discovery messages. Moreover, these schemes work in unlicensed bands where interference is not well controlled, and therefore, safety applications should not make use of these technologies. For these drawbacks, operators do not traditionally accept this type of communications and are more prone to new systems in which QoS can be guaranteed under the control of the cellular network. In this framework, the 3GPP started to study proximity-based services under the name of Study on LTE Device to Device Proximity Services, whose agreements were included in the technical report [43]. The two primary aspects of D2D specified in this report are discovery and direct communication. In [44], the 3GPP identified a set of use cases for the LTE D2D study. These use cases do not only focus on commercial services but also on public safety. In fact, in January 2011, the Federal Communications Commission (FCC) adopted the use of LTE to improve the communication of the first responders in large-scale emergencies and enable to share information between departments. In the Mobile World Congress 2014, Qualcomm developed a proof of concept of its D2D technology called LTE-Direct [45]. From this first attempt, Deutsche Telecom made a trial in 2014 of its prototype LTE Radar. Furthermore, the car industry is starting to show interest in LTE D2D communications to serve their demands for future Vehicle to Vehicle (V2V) communications. 


\subsubsection{Scenarios}

Figure 2.4 shows four scenarios under study for the LTE-based D2D communications, categorized according to the coverage status, i.e., out-of-coverage, partial coverage and in coverage. Within the in-coverage scenario, single cell and multi-cell scenarios are also studied. In this case, an in-coverage user can receive Primary Synchronization Signals (PSSs), Secondary Synchronization Signals (SSSs) as well as Physical Broadcast CHannel (PBCH) from a cell. These signals allow the base station to coordinate the communication between devices simplifying, thus, the standardization. Due to this fact and time constraints, the $3 \mathrm{GPP}$ focused its priority on in coverage scenarios.

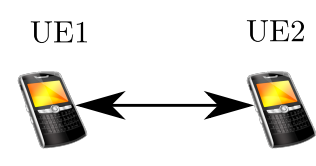

Out-of-coverage

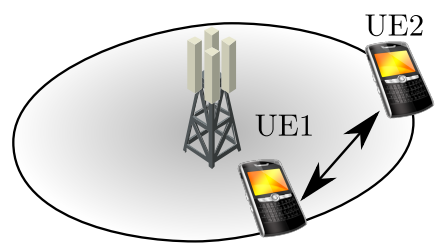

In-coverage single cell
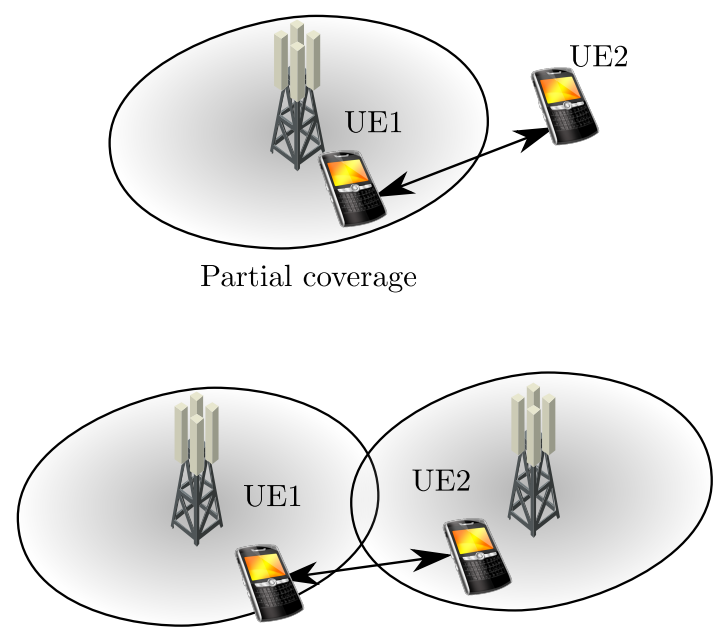

In-coverage multiple cell

Figure 2.4: D2D scenarios.

\subsubsection{Evaluation methodology, metrics and channel mod- eling}

To study the above-described scenarios, the 3GPP defined a standard method of evaluation of the general (i.e. commercial) and public safety use cases [43]. Different evaluation assumptions have been established concerning the layout, carrier frequency, system bandwidth, network synchronization, UE mobility, traffic models, and UE dropping methods, among others [43]. Two out of six layouts incorporate the use of Remote Radio Head ( $\mathrm{RRH})$ or indoor hot zones. Another layout utilizes a dual stripe modeling representing residential 


\section{CHAPTER 2. FEATURES AND CHALLENGES OF THE COOPERATIVE 5G SCHEMES}

or commercial buildings. The UE mobility models focus on pedestrian speeds for typical scenarios and up to $60 \mathrm{~km} / \mathrm{h}$ in public safety scenarios. Note that both transmitter and receiver could be moving in opposite directions such that the maximum relative speed could be $120 \mathrm{~km} / \mathrm{h}$.

The metrics that evaluate these scenarios are divided into two categories based on the two primary aspects of LTE-based D2D communication, i.e., discovery and direct communication. On the one hand, the discovery metrics are the number of discovered UEs as a function of time, the probability of discovery versus distance, throughput loss of the LTE network and power consumption [43]. On the other hand, the communication metrics are based on user throughput for full buffer, File Transfer Protocol (FTP), and Voice over IP (VoIP), mean throughput versus distance, throughput in the LTE network and power consumption [43].

Accurate channel models are needed to evaluate the different operator proposals. Owing to time constraints, 3GPP decided to use pre-existing channel models from European projects such as Wireless World Initiative New Radio (WINNER) II and WINNER+, or from institutions like the ITU [46], to simulate large and small-scale fading effects.

\subsubsection{Physical layer aspects}

\section{Spectrum}

One of the main aspects of the design of D2D is the management of resources [47]. In theory, in the LTE Frequency Division Duplexing (FDD) network, the communication among users could occur in DL or Uplink (UL) resources. However, resource allocation in DL spectrum presents the following drawbacks. First, the transmission on DL resources forces the implementation of a separate transmission/reception chain in the radio frequency front-end at the user equipment. Thus, the complexity of the receiver would be considerably increased. Second, if a D2D user transmits on the DL spectrum, cellular DL users would be interfered reducing system performance. Time Division Multiplexing (TDM) techniques could partially solve this drawback. However, this interference keeps being a problem unless there was a synchronization method among cells. Moreover, the eNB is often located far away from the D2D users, and therefore, the interference could be negligible if UL resources were used. Moreover, even though the D2D link was close to the BS, the complexity of this BS could be increased and the interference, thus, reduced. Third, DL resources are more utilized than UL resources, and a new D2D resource allocation would imply a general reduction in the data rate. 
For the above-mentioned reasons, the 3GPP preferred D2D operation in UL bands in the FDD case [43]. Furthermore, it is assumed that, in the same frequency band, a D2D cannot transmit and receive at the same time, i.e., in the user, like the fixed relay, a half-duplex mechanism is implemented.

\section{Synchronization}

Similarly as the synchronization in LTE uplink, D2D communication must be synchronized to avoid interfering (and being interfered by) other receivers. The 3GPP is studying three types of synchronization sources. First, the most natural synchronization source consists in using the timing of one, or, even more, eNBs, through Device-to-Device Synchronization Signal (D2DSS). If the synchronization source is an eNB, the signal is composed of Release 8 PSS/SSS. In this case, in coverage users are directly synchronized since the reference is fixed by the base station and all users are synchronized with the same signal. Second, for out-of-coverage scenarios, one or several UEs can transmit D2DSSs in a master-slave structure, where the master, also known as synchronization cluster head, sends the synchronization signal, and it is used as a reference by the slave UE. Third, the synchronization is set by an external source such as Global Navigation Satellite System (GNSS). These signals are governed by some priority rules, like for instance that a eNB has higher priority than a cluster head, and an in-coverage UE has higher priority than an out-of-coverage source. Lastly, the synchronization source selection is based on the received D2DSS quality. On the contrary, if no signal is received, the user could transmit its D2DSS without any reference.

These three synchronization signals together with the prioritization rules are used to achieve system-wide synchronization through centralized or distributed procedures. In the former, one network element, either an eNB in in-coverage scenarios or a cluster head in out-of-coverage scenarios, sets the synchronization for a set of users. This approach guarantees the synchronization among this network element and the different devices that are camping within the same cell or cluster head coverage. However, UE to UE synchronization is not guaranteed in scenarios where UEs are synchronized under different D2DSS. Note also that the different sources could interchange information or even directly initiate a joint synchronization procedure. In addition, some in coverage synchronized UEs could work as a relay to send the D2DSS to UEs in neighbouring cells or to out-of-coverage UEs. In the same way, some out-of-coverage synchronized UEs could work as relays to increase the synchronization range of the cluster head. Regarding the second procedure, the distributed one, there does not exist any network element that controls the synchronization of the users. Rather, each 


\section{CHAPTER 2. FEATURES AND CHALLENGES OF THE COOPERATIVE 5G SCHEMES}

UE sends synchronization signals to establish a common time and frequency synchronization.

\subsubsection{Discovery}

One of the main aspects of D2D communication is the discovery of devices that are located in the surroundings. Logically, not all devices are authorized to decode some discovery signals. This discovery can be useful to implement discovery for a limited group of users. It is important to emphasize that discovery is an additional characteristic of D2D communications, and it is not required for the direct communication among devices. Discovery in the 3GPP is defined for both general and public safety scenarios. Discovery use cases are defined for all D2D scenarios depicted in Figure 2.4, that is, for out-ofcoverage, partial coverage and in-network coverage. In Release 12, discovery is only available within network coverage.

\section{Discovery types}

There exist two discovery methods based on the allocation of resources. In the former case, known as Type 1, a resource pool is shared between all users or a group of them. Resources are periodically allocated during a set of consecutive subframes. Within this period, resources are divided into equal sized time-frequency resource blocks known as discovery resource. Thus, the UE transmits on a randomly selected discovery resource. Each UE will listen and transmit discovery signals under the half-duplex constraint. For an in-coverage UE, resources are assigned by the eNB using Radio Resource Control (RRC) signaling or through System Information Block (SIB).

The latter case, if resources are assigned user by user, is referred to as Type 2. Within this last category, the 3GPP divides discovery into Type $2 \mathrm{~A}$ and Type 2B. In the first instance, resources are assigned for each particular transmission. In the second case, resources are allocated semi-persistently. In the Type 2B, only RRC CONNECTED UEs can request resources for transmission. These resources are maintained until either the UE enters in RRC IDLE state or the eNB forces it to release the resources.

\section{Signal design}

The discovery transmission consists of a MAC Protocol Data Unit (PDU) mapped into a subframe similar to the uplink subframe for transmitting Physical Uplink Shared CHannel (PUSCH). Figure 2.5 shows an example of discovery subframe structure for a normal cyclic prefix. In this structure, a gap 


\subsection{D2D communications}

is introduced at the end of the subframe to allow the transition of becoming signal transmitter or receiver.

\subsubsection{Direct communication}

Direct communications among devices can provide benefits to users when they are in proximity. In particular, it is expected to increase the user throughput and spectral efficiency together with extended coverage. Moreover, UE power consumption could be significantly reduced with D2D communications.

\section{D2D communication types}

3GPP categorizes three types of D2D communications:

- Unicast. It consists in a one to one path that offers reduced latency and high data rates.

- Groupcast. It provides a communication between one to many UEs. In the 3GPP simulations, a groupcast communication in the public safety scenario is composed of ten users (one transmitter and nine receivers).

- Broadcast. It provides a communication among one to all UEs. In the 3GPP simulations, a broadcast scenario is composed of a variable number of users.

\section{Resource allocation}

The 3GPP has defined two resource allocation types for D2D communications [43], i.e., Mode 1 and Mode 2. In the first case, the eNB or the Release 10

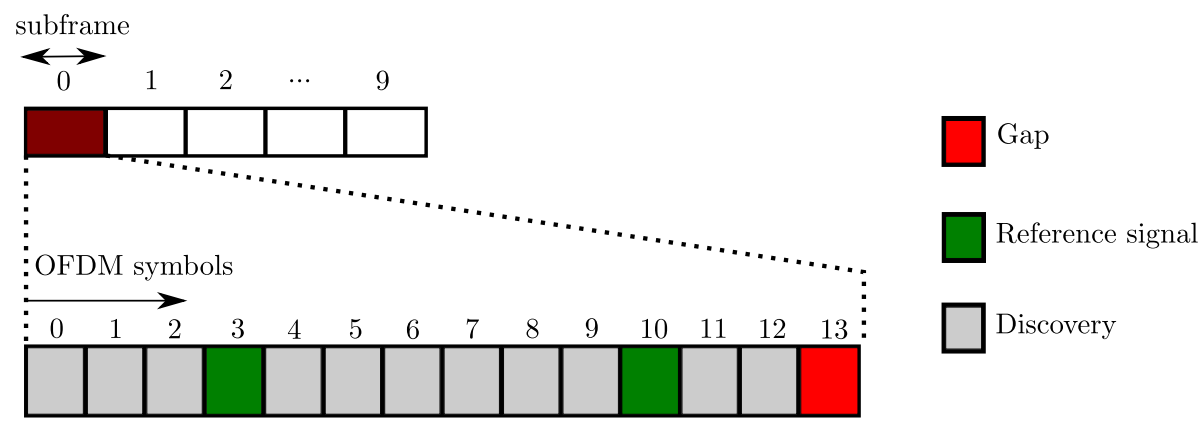

Figure 2.5: Discovery subframe. 


\section{CHAPTER 2. FEATURES AND CHALLENGES OF THE COOPERATIVE 5G SCHEMES}

relay node schedules the specific resources for data or control information. In Mode 1, an RRC CONNECTED UE sends a scheduling request and a buffer status report with how much data is in the buffer to send out. With this approach, it is foreseen minimal standard changes at expenses of some additional signaling overhead. In the second type, each UE selects from a resource pool the resources to be used. The access to this resource pool could be based on a contention process or in a semi-static or pre-configured way. These two last options are under discussion in the 3GPP. Users camping on a cell must support at least the first choice. The second option is designed for cell-edge users or out-of-coverage users.

\subsection{V2X communications}

The 3GPP decided to separate the study item about vehicle communications from the study item of D2D communications and add new functionalities, developing a new Study on LTE Support for V2X Services ${ }^{2}$. Note that ProSe have been designed for pedestrian mobility speeds. V2X communication is the interchange of information between a vehicle and an entity that could affect the vehicle or vice versa. This information can be used for reasons such as driver assistance, speed adaptation, traveler information, and navigation.

\subsubsection{Traditional vehicular protocols}

Academia and industry have developed the idea of wirelessly connecting vehicles for the last twenty years, where the main regions of research and application are the United States, Europe, and Japan. The main standardization bodies in these regions are IEEE or Society of Automotive Engineers (SAE), European Telecommunications Standards Institute (ETSI) and Association for Radio Industry and Business (ARIB), respectively. The IEEE 802.11p is an amendment to the IEEE 802.11 standard to support Wireless Access in Vehicular Environments (WAVE). However, this standard does not support neither authentication nor association among the vehicle and the infrastructure, and upper layers must support this functionality. Based on the IEEE 802.11p standard, the ETSI created a set of standards known as Intelligent Transportation Systems G5 (ITS-G5). This standard covers the two lowest layers in the protocol stack, i.e., physical layer and data link layer (from bottom to top). This last layer is divided into two sublayers: MAC and logical link layer (from bottom to top). The physical and MAC layers of this standard can be based on the IEEE 802.11p standard, which is currently the preferred option.

\footnotetext{
${ }^{2}$ V2X stands for Vehicle to Everything.
} 
Although the IEEE 802.11p protocol has been proposed for European and American standards, some V2X use cases require infrastructure assistance for communications, using Road Side Units (RSUs), small cells or relays. As a solution to this problem, the 3GPP could support the ETSI V2X services in their cellular systems. In this sense, the ETSI is encouraging the $3 \mathrm{GPP}$ to study the integration of its ITS-G5-air interface with 3GPP cellular solutions and invites the $3 \mathrm{GPP}$ to inform them of the progress of the LTE support for V2X services [48]. The 3GPP, on the other hand, agrees to support several service layers including ETSI Intelligent Transportation Systems (ITS) [49].

\subsubsection{Communication types}

The study of vehicular communications does not only cover the communication among vehicles, that is, V2V services, but also Vehicle to Infraestructure (V2I) and Vehicle to Pedestrian (V2P) services. Figure 2.6 describes the general diagram of the V2X services. For all these services, one party of the communication is a UE, and the other party is another UE, a RSU or a pedestrian that uses the Evolved UMTS Terrestrial Radio Access Network (E-UTRAN). It is also included the concept of Vehicle to Network (V2N) service, where the communication takes place via an LTE network between a UE and a serving entity. To date, V2V services are prioritized against other services.

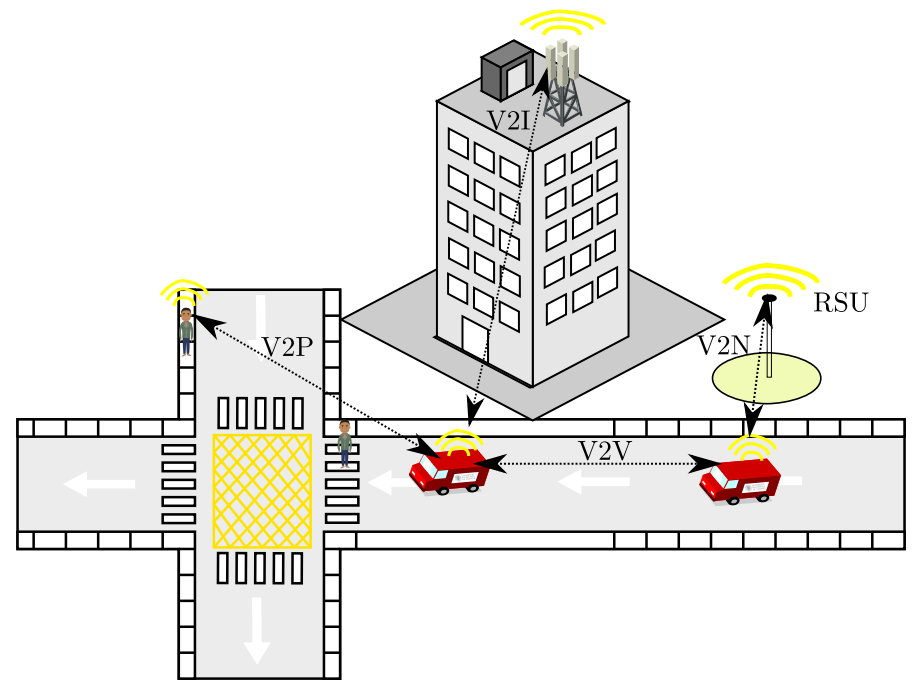

Figure 2.6: Types of V2X communications: V2V, V2I and V2P. 


\section{CHAPTER 2. FEATURES AND CHALLENGES OF THE COOPERATIVE 5G SCHEMES}

\subsubsection{Use cases}

The 3GPP has defined 24 use cases that cover the different services about V2X. In particular, for each use case, conditions to support the V2X services, service flows of the implied entities and potential requirements to carry out the communication are detailed [50]. Some use cases are related to forward collision warning messages, control loss warning, automated parking system, warning to pedestrian against pedestrian collision, and traffic flow optimization.

\subsubsection{Design}

The 3GPP is working on different considerations of V2X services regarding E-UTRAN coverage, spectrum, security, and mobility. The various parameters of security are related to anonymity, integrity protection, confidentiality, and finally, authentication and authorization for licensed spectrum. In [50], an example of link-layer parameters for V2X services is shown. The scenarios under study are suburban, highway, urban with Non Line of Sight (NLoS), urban intersection, and campus shopping area. The relative velocity between two UEs supporting V2X services are 30,100 and $280 \mathrm{~km} / \mathrm{h}$. Note that some of these speeds pose a challenge in the behavior of V2X services, especially for high-order MIMO, where coherent systems often reduce the number of training symbols to increase the obtained data rates.

\subsection{Discussion and future trends}

This chapter has presented an overview of the main technologies that are being proposed by the research community and the main standardization organisms for next-generation wireless systems. Traditional communication schemes such as LTE are based on coherent communications in which a set of pilot symbols are sent to the receiver with the objective of estimating the channel and performing a coherent detection. This detection method presents a set of implementation drawbacks that needs to be analyzed.

Firstly, in high-speed scenarios these schemes suffer from severe performance degradation, especially in high-order MIMO systems. In fact, in LTE, with four transmit antennas, the amount of training in the third and fourth antenna is reduced by half as compared to the other two antennas. This pilot allocation is due to the fact that the use of four transmit antennas is designed for slow-speed scenarios and high SNR. However, in moving networks, this pilot allocation is not adequate since the channel estimation of the eNB-RN link becomes involved. However, the RN-UE link remains static, and its esti- 
mation could be assumed perfect. Thus, the coherent communication poses a bottleneck due to the eNB-RN link, and the implementation becomes difficult.

Secondly, in cooperative networks, and due to the broadcast nature of communications, performing coherent detection in relay networks consumes significantly more resources than point-to-point communications since the destination must obtain the instantaneous relay-destination, source-destination, and if possible, the source-relay CSI. Note that one of the reasons to get the quality of the source-relay link at the destination could be to instruct the source and the relay on bandwidth and power allocation functions. This fact is aggravated when increasing the number of relays since the channel estimation overhead and the receiver complexity increase proportionally. Moreover, to acquire an accurate CSI, the channel must vary slowly, which limits the mobility of the source, relay, and destination. To overcome these drawbacks, the transmitter and the relay may use a transmission scheme that does not require the CSI knowledge at any of the communicating points. This signaling scheme is often referred to as non-coherent communication and will be studied in detail in Chapter 4.

In addition to fixed relays discussed so far, current research focuses on a new paradigm of the wireless network in which UEs become MRs and assume an active role in the network to guarantee the ubiquity of high-quality services. Although the concept of device-to-device communication to help other users within a cellular system is not entirely a new concept, e.g., it was discussed by $3 \mathrm{GPP}$ under the name of Opportunity Driven Multiple Access [51], this is a challenging scenario with many open questions that need to be solved. Among others, important research areas for the future of mobile relaying are:

- The design of forwarding strategies that allow for the integration of cooperation in conventional point-to-point communications.

- The design of algorithms robust against imperfection on CSI at the transmitters and relays.

- The solution of the trade-off between macro-diversity gains and the additional signaling overhead required by cooperative relaying.

- The proper relay selection and its transmitted power.

- The definition of incentives that do not require for additional signaling in the network. 
CHAPTER 2. FEATURES AND CHALLENGES OF THE COOPERATIVE 5G SCHEMES 


\section{Chapter 3}

\section{Network-layer solution: MIH for mobile relaying}

\subsection{Introduction}

Technologies such as LTE-Advanced [52] or WiMAX IEEE 802.16m have emerged as promising standards. These technologies are designed to deliver peak data rates above 1 Gbps with a bandwidth of up to $100 \mathrm{MHz}$ for low mobility users and $100 \mathrm{Mbps}$ for high-speed users [53]. However, high data rates can be only provided within the vicinity of the BS. Hence, it would take a large number of BSs to cover a given area with high data rates. Certainly, this means a deployment cost that operators cannot afford. This situation motivated the study and development of relays as a means to increase the coverage in indoor scenarios and public transportation vehicles (trains, buses, etc.). The use of fixed cooperative relays has already been introduced in WiMAX Mobile [54] -IEEE 802.16m standard - and in the 3GPP LTE-Advanced standard. Although the results of this Thesis are valid for both systems, this chapter focuses on the 3GPP solution.

A further step towards full integration of relaying is the use of mobile terminals as relays, which is known as mobile relaying. The optimal relay selection is of great interest, and signaling overhead cannot be underestimated. An optimal routing mechanism must be defined so as it can dynamically adapt to changes in the system while involving the least possible signaling overhead. To date, the LTE-Advanced standard contemplates the use of fixed relays as a means to increase coverage and data rates. However, the MR concept has still not been included in the timeline of the 3GPP specification. Only some studies are being 
performed to relay some synchronization signals with direct D2D communication among users. However, in this study, it is still necessary to define some mechanism of MR selection. Within this framework, this chapter proposes the use of IEEE 802.21 MIH standard to support the relay selection process. MIH standard is an application protocol that can be easily implemented on mobile and network devices. MIH defines a set of entities and messages that facilitate the interchange of information between network nodes. It allows having local and remote reports on link status so that decisions can be made dynamically in the access network. Besides, it provides intelligence to both the physical and MAC layers and network information to upper layers [19]. Initially, this standard was designed to optimize handovers in heterogeneous networks, but the Thesis proposal is to extend its usage to this new application field.

Therefore, this chapter proposes a new use of MIH signaling to the management of multiple radio interfaces in a cellular system assisted with MRs. The system scenario comprises an LTE-Advanced network where MNs have another WiFi radio interface for ad-hoc communications. This assumption is fully aligned with current technological trends since both radio interfaces are expected to coexist in the coming mobile devices. This chapter aims at analyzing the signaling requirements and provides not only an optimal configuration but also mechanisms for reducing the overhead. Moreover, we analyze to which extent mobile relaying can support the deployment of lightweight networks.

The rest of the chapter is structured as follows:

- Section 3.2 shortly analyzes the enabling technologies necessary to implement the chosen solution.

- Section 3.3 describes the proposed relay selection mechanism based on MIH signaling.

- Section 3.4 presents the simulation methodology used to assess the performance of the new algorithms.

- Section 3.5 discusses the main parameters of the proposed relay selection algorithm. Furthermore, it includes a simplified high-level financial analysis of the an LTE-Advanced system with the use of MRs.

- Section 3.6 outlines the key findings of the study.

\subsection{Current technological scenario}

To use MIH signaling to manage the routing of packets dynamically it is necessary to define a set of technological tools that support the proposed solution. The following sections describe the basis of these technologies. 


\subsubsection{LTE-Advanced}

$4 \mathrm{G}$ cellular systems are being designed to increase the coverage and user data rates. The spectrum for $4 \mathrm{G}$ systems can be divided into five bands: the 450 $\mathrm{MHz}$ band, the Digital Dividend (DD) band around $700 \mathrm{MHz}$, Advanced Wireless Services (AWS) band between $1.7-2.1 \mathrm{GHz}$, the $2.5 \mathrm{GHz}$ and, finally, the C-band around $3.5 \mathrm{GHz}[55]$. Note that the DD band is the spectrum available after the switch-over from analog to digital television. As can be seen, most of these bands are above $2 \mathrm{GHz}$, where propagation loss exponent is higher and therefore, under these conditions, traditional cellular architectures require a higher density of BSs. Obviously, increasing the density of BSs is very costly for operators, and other alternatives are preferred. One solution may be to increase the allocated bandwidth or spectral efficiency. Indeed, this chapter will show that mobile relaying implies a significant increase in spectral efficiency.

In a scenario with relays, there are three types of link: a BS to user link, a BS to relay link (also known as backhaul), and a relay to user link. It is worth noting that the relay node is connected via the wireless link to the radio access network through the BS or donor cell.

\subsubsection{IEEE 802.11n}

One of the most pervasive wireless technologies for use in homes, offices, and other multiple scenarios is the IEEE 802.11 technology. 802.11n offers peak data rates of more than $100 \mathrm{Mbps}$. The used bandwidths are 20 or $40 \mathrm{MHz}$ in the bands of 2.4 or $5 \mathrm{GHz}$ [56]. The physical layer data rate can even exceed 300 Mbps provided $2 \times 2$ spatial multiplexing and $40 \mathrm{MHz}$ bandwidth. This high performance is achieved through a range of new technological solutions such as MIMO schemes, spatial multiplexing, spatial mapping (including beamforming), STBC, and Low-Density Parity Check (LDPC) coding.

\subsubsection{MIH}

The IEEE 802.21 MIH is specially designed to perform handovers between different IEEE 802 architectures (802.3, 802.11, 802.16) [19]. However, other 3 GPP technologies such as LTE-Advanced can be included in its operation. Next-generation handsets are ideal candidates for installing and using the MIH protocol since they have multiple radio access interfaces (UMTS, LTE, WiFi, etc). Moreover, the MIH protocol can easily be included in the application layer.

The MIH signaling is a protocol that is based on the exchange of messages reporting a subset of PHYsical (PHY)/MAC layer events between entities called Media Independent Handover Function (MIHF). The MIH functions 
provide Media Independent Handover Event Services (MIES), Media Independent Handover Command Services (MICS), and Media Independent handover Information Services (MIIS). Figure 3.1 shows the main entities in the MIH protocol, as well as the events and commands generated by these entities. The MIES detects changes in the link layer and initiates events from both local and remote interfaces. The MICS offers the MIH-User control over the connection properties that are relevant to the handover. Finally, the MIIS provides information on different heterogeneous networks. There are specific events to notify link power going down, link disconnection, degradation of the channel, link handover is imminent, and so on.

The different events and commands are defined in the MIH standard [19]. Depending on where the information is originated, the MIHF entity could receive or transmit reports on the configuration and condition of the radio access networks the $\mathrm{MN}$ is detecting. If the information is obtained remotely, the local MIHF entity receives information from the remote MIHF entity that is located in the network. However, when information is received from the lower layers of the protocol stack, this is obtained through service primitives that define the interface. For example, if the signal strength received by a remote entity falls below a threshold, then lower layers detect it and send a Link Going Down (LGD) event to the MIHF entity. This entity communicates with the remote MIHF through Remote MIH Events. Once the event reaches the local MIHF entity, it is forwarded via a MIH event to the MIH-User. In the same way as with events, local MIH-User can send a command to make lower layers perform a particular operation. For example, thresholds can be adjusted, the active link quality can be measured according to SINR, BER, etc. Finally, to integrate $\mathrm{MIH}$ within $3 \mathrm{GPP}$ systems, there is no need for new protocols to access MIHF services. These services can be mapped to those already existing in $3 \mathrm{GPP}$.

\subsubsection{Localization}

User positioning can significantly reduce the complexity of the relay selection process. LTE-Advanced specification considers MN localization through the LTE Positioning Protocol (LPP) and LTE Positioning Protocol annex (LPPa) [57, 58]. Several positioning methods are mentioned in the standard, namely: Assisted-Global Navigation Satellite System (A-GNSS), Observed Time Difference of Arrival (OTDoA), and Enhanced-Cell ID (E-CID). All of these positioning methods are based on measurements collected by the MN or the BS. The Mobility Management Entity (MME) is the entity that receives the request for the localization of a MN from another entity such as a BS. Then, the MME sends a location service request to the Enhanced Serving Mo- 


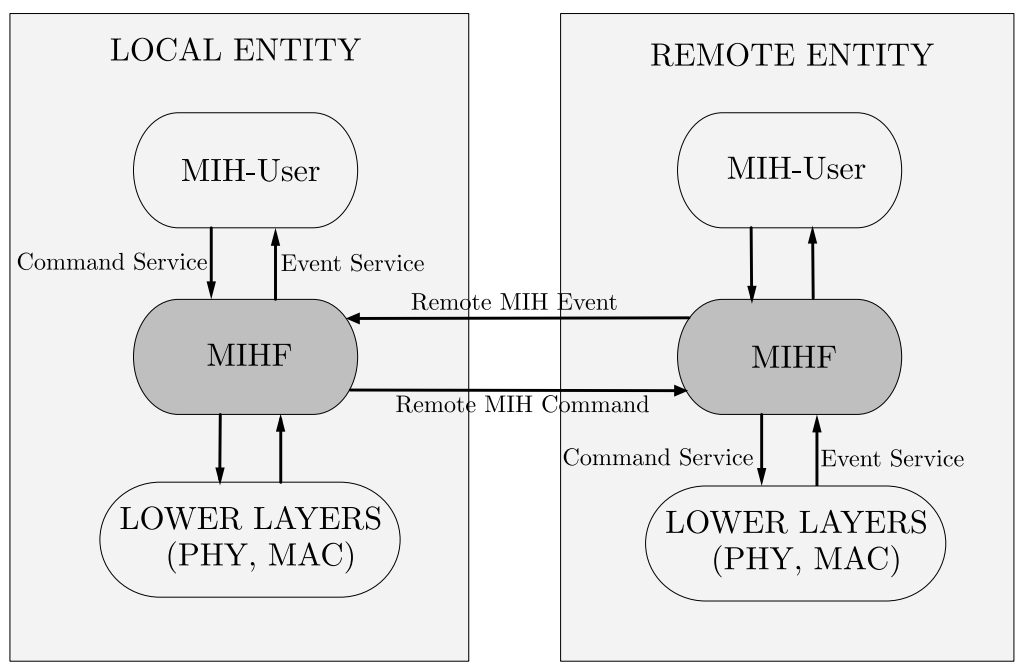

Figure 3.1: Media independent handover operation.

bile Location Centre (E-SMLC), which will execute the positioning procedure through LPP and LPPa protocols. While the LPP is a point-to-point protocol that exchanges LPP messages between the UE and E-SMLC, the LPPa allows the communication between the eNB and E-SMLC. The study of different positioning methods is outside the scope of this Thesis. However, it is worth noting that in LTE-Advanced, advanced location methods are available and can be used by the relay selection mechanism proposed in this chapter.

Concerning the signaling overhead of user positioning, note that the periodicity of LPP methods must be higher than $0.5 \mathrm{~s}$, which is the regular period of measurement reports sent by LTE mobile nodes. Considering that the MIIS entity is independent for each BS, the database burden is limited to hundreds of inserts per second, which is an order of magnitude lower than the current capacity of commercial databases.

\subsection{MIH-driven relay selection mechanism}

Cell-edge users suffer a reduction in data rates as compared with users who are close to the BS because the SINR is usually lower, creating some unfairness among UEs due to their location. Thus, considering that the spectral efficiency requirements set by the ITU for $4 \mathrm{G}$ technologies are very demanding [55], both IEEE and 3GPP have decided to use relays to increase the cell-edge 
user spectral efficiency. The use of relays decreases the transmission range, increasing the probability of receiving the data correctly, allowing higher data rates than those achieved without relays.

Figure 3.2 shows the scenario under study. Three types of nodes are distinguished: a BS, MNs, and MRs. The MN that is experiencing bad channel conditions is referred to as Solicitor Mobile Node (SMN). In this scenario there exist MNs receiving from the BS (active nodes) and MNs in the idle mode (passive nodes), which are the only ones that can relay signals from or to the SMN. Both MNs and MRs have two interfaces: an LTE-Advanced interface to communicate directly with the BS and a WiFi interface for the MN to MR link. It is considered that the link between the MR and the MN is implemented with the IEEE 802.11n standard [56]. As a baseline, all MNs are connected with LTE-Advanced unless the use of mobile relaying implies higher data rates.

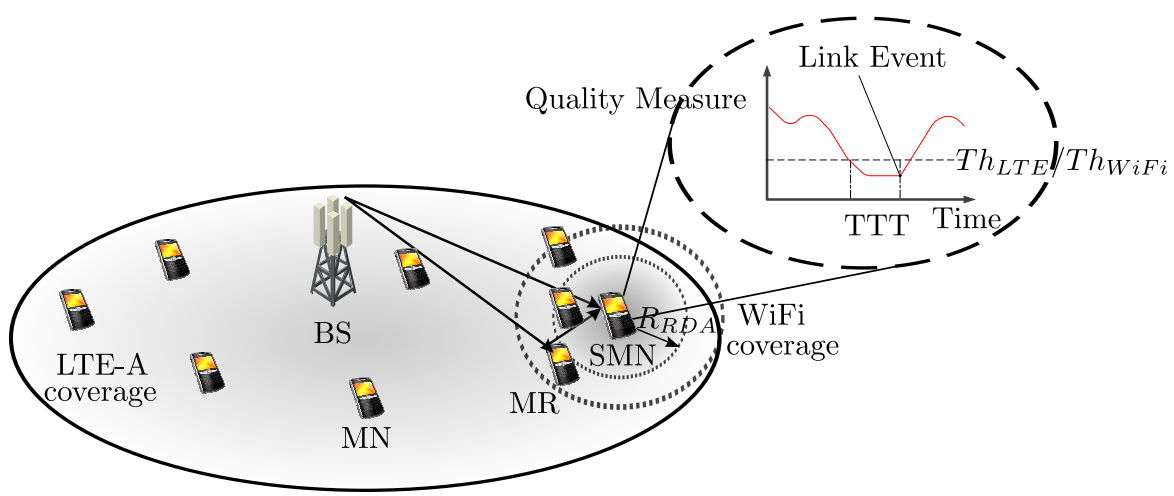

Figure 3.2: Simulation scenario under study.

The MIDRES mechanism proposed in this Thesis uses MIH signaling to define a routing and handover mechanism for mobile relaying in $4 \mathrm{G}$ mobile systems. In this chapter, it is assumed that nodes can have both LTE-Advanced and WiFi radio interfaces simultaneously active. When handover occurs, the packet route switches from one technology to another and the old technology transits to the idle state. As shown in Figure 3.2, if a MN connected to LTE-Advanced experiences a SINR level below the quality threshold, $T h_{L T E}$, during Time To Trigger (TTT) seconds, then a LGD event is sent from lower layers to the MIH-User situated in upper layers. Similarly, a LGD event could be initiated and sent to the MIH-User in the MR. These events initiate the handover procedure. Note that the SINR is a measure of the signal quality typically utilized by the UE to calculate the Channel Quality Indicator (CQI). 
The UE sends this value to the eNB as an indication of the data rate that can be supported in the downlink channel. The CQI sets the highest Modulation and Coding Scheme (MCS) to decode the transport blocks with an error rate lower than $10 \%$. The CQI values are carried by PUCCH or PUSCH for downlink scheduling and link adaptation. On the other hand, in the WiFi link, the minimum receiver sensitivity, $T h_{W i F i}$, is defined as the minimum signal level to demodulate with a Packet Error Rate (PER) less than 10\%. This minimum level is the average power per receive antenna. Note also that while $T_{L T E}$ is defined in $\mathrm{dB}$ units, $T_{W i F i}$ is defined in $\mathrm{dBm}$.

It is assumed that all MNs and the BS are MIH-Users and implement MIHFs. In the BS the MIHF is also known as Point of Service (PoS). As shown in Figure 3.1, MIH communication among entities such as BSs or MNs is carried out through MIHFs. The destination of an event is established with a subscription mechanism that enables MIH-Users to subscribe to particular event types located either in the same equipment or remote nodes. The main difference between the MIHFs of BSs and MNs is that MNs announce events from lower layers to the subscribed entities, whereas the BS initiates and manages possible handovers among technologies.

In mobile relaying three different situations may occur:

1. Situation 1. The SMN is connected to LTE-Advanced, and the SINR level is lower than $T h_{L T E}$. In this case, MIDRES initiates the handover procedure to select the most appropriate MR.

2. Situation 2. The mobile relaying for a $\mathrm{MN}$ is active, and either the LTE-Advanced or the WiFi interface goes below the quality threshold. Then, MIDRES initiates the procedure looking for a new available MR or making a handover back to LTE-Advanced.

3. Situation 3. The mobile relaying for a $\mathrm{MN}$ is active, and the LTEAdvanced SINR level of the MN is higher than the quality threshold ${ }^{1}$. In this case, MIDRES initiates the procedure searching for the best connection configuration.

\subsubsection{Situation 1}

Figure 3.3 shows the exchange of messages when mobile relaying provides better throughput to the SMN than the direct LTE-Advanced link. The figure also illustrates the connection state for LTE-Advanced and WiFi. In this situation, MIDRES procedure includes the following phases:

\footnotetext{
${ }^{1}$ It is considered in this chapter that this threshold is again $T h_{L T E}$.
} 
CHAPTER 3. NETWORK-LAYER SOLUTION: MIH FOR MOBILE RELAYING

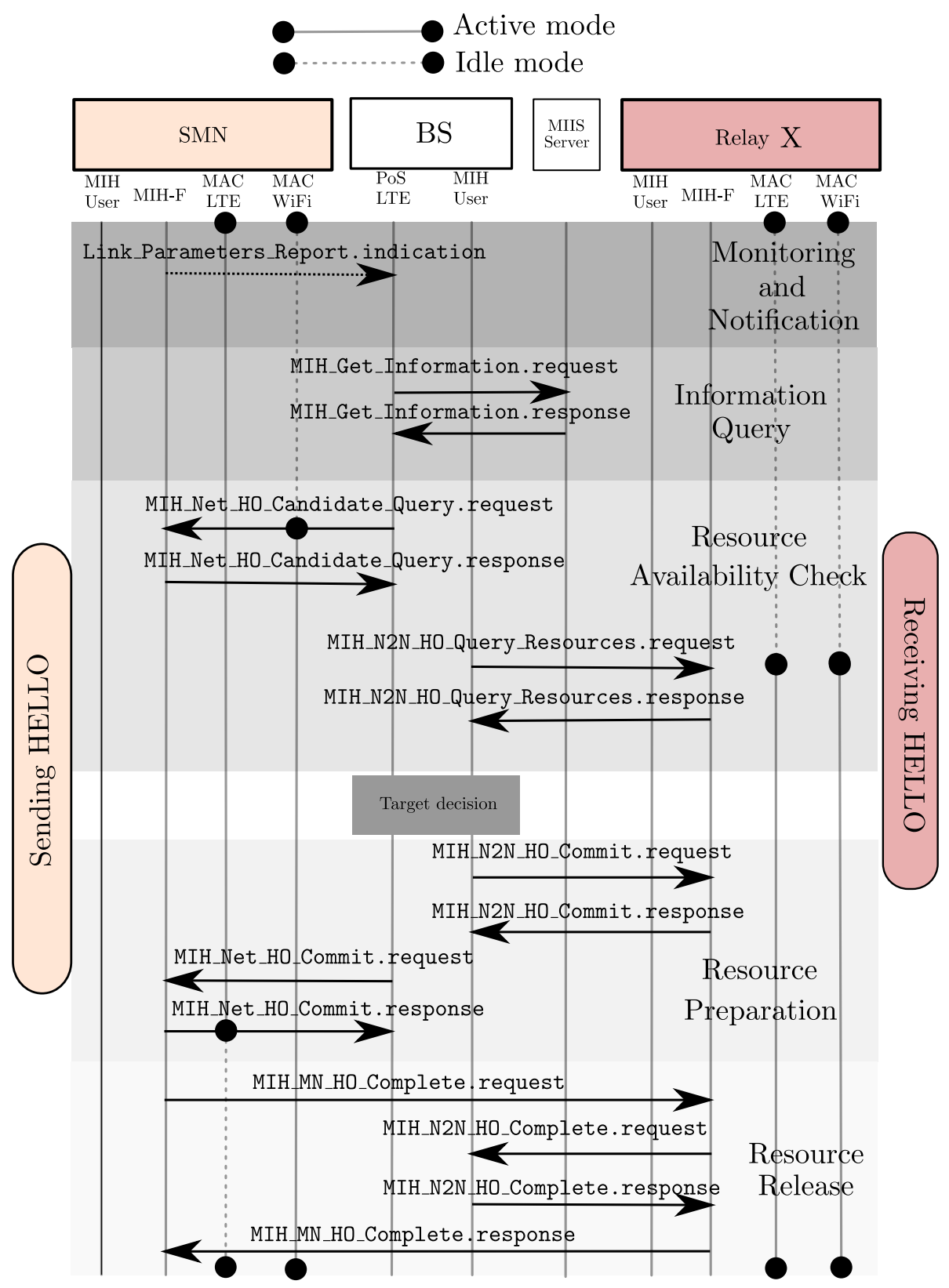

Figure 3.3: MIDRES procedure to configure a MR. 
1. Monitoring and Notification. When the SMN detects that the SINR level is below $T h_{L T E}$ during TTT seconds, the lower layers send a LGD event to the MIH-User that forwards this event to the BS with the Link_Parameters_Report.indication message.

2. Information Query. The BS MIH-User sends a Get_Information.request message towards the Information Server (IS) asking which relay nodes are candidates. The BS could attach a radius called $R_{R D A}$, centered at the indicated location. In that case, the IS would decide upon this radius. At this point, the IS can run the proposed Relay Discrimination Algorithm (RDA) to reduce MIH signaling. Basically, the RDA consists in selecting those cooperative MNs that are at a distance less than $R_{R D A}$ from the SMN. Note that the IS can periodically estimate the location of all MNs using E-SMLC described in Section 3.2.4. Finally, the IS entity responds with a Get_Information.response message that includes the list of candidate MRs.

3. Resource Availability Check. After the confirmation of available relays, the BS sends a MIH_Net_HO_Candidate_Query.request to the MN providing the list of candidate MRs. The MN indicates the required resources in the MIH_Net_HO_Candidate_Query.response message. At this stage the SMN activates its WiFi interface and sends periodic HELLO messages so that the candidate MRs can detect its presence. Next, the BS polls all MR candidates on the feasibility of the connection by sending a MIH_N2N_HO_Query_Resources.request. Once the candidates receive this message, they activate their WiFi interface and measure the link quality with the SMN, analyzing the received HELLO messages power and therefore the link throughput. After this measure, each relay node sends the result to the BS in a MIH_N2N_HO_Query_Resources.response message through the LTE-Advanced interface. Note that, in addition to this information, the BS can estimate the mean throughput of its link with the MR since the LTE-Advanced connection is active at this stage. Using these data, the BS decides on the best connectivity option, that is, remain connected to LTE-Advanced or use a MR.

4. Resource Preparation. If the best option is to use a relay, the process continues. The BS sends a MIH_N2N_HO_Commit.request message to the selected MR marking the beginning of the routing process. The relay answers with a MIH_N2N_HO_Commit.response. From this point on, the relay will route all packets from the BS to the SMN. For all purposes, the relay will be connected with the $\mathrm{BS}$ as another user. To distinguish the final destination (it may be that the relay receives a packet for itself), 


\section{CHAPTER 3. NETWORK-LAYER SOLUTION: MIH FOR MOBILE RELAYING}

the relay will only have to inspect the Internet Protocol (IP) packet's destination address. Of course, for this to be viable, it is necessary that the relay possesses routing capabilities. In the same way, the BS informs the SMN about the decision with a MIH_Net_HO_Commit.request message to commit handover towards the specified relay. The new radio interface connection is established, and the MN sends a MIH_Net_HO_Commit.response to the BS.

5. Resource Release. In the last stage mobile relaying must be confirmed. The SMN sends a MIH_MN_HO_Complete.request to the selected relay that answers with a MIH_MN_HO_Complete.response. The completion is also reported to the $\mathrm{BS}$ with a MIH_N2N_HO_Complete.request and its corresponding response message.

At this point it is worth discussing another alternative of MIDRES in which the SMN measures the quality of the candidate MRs. Note that the decision of making the MRs measure the channel is aimed at reducing the handover delay. With the current proposal of MIDRES, the SMN is the only one sending HELLO messages, and this reduces the contention problems. With the other alternative, all relay nodes should content to seize the channel and, therefore, there would be potential collisions and hidden node problems. As a consequence, the time required for the handover will be much higher.

\subsubsection{Situation 2}

Figure 3.4 shows the exchange of messages when, in an active mobile relaying, the power level of the WiFi radio interface falls below the threshold ${ }^{2}$, $T h_{W i F i}$. When this situation happens, the MR sends to the BS the corresponding Link_Parameters_Report.indication message. From this moment, all phases of the MIDRES procedure are identical to the Situation 1, except the resource release phase. Now the BS sends a MIH_N2N_HO_Complete.request to the old relay to release the assigned resources. The old relay answers with a MIH_N2N_HO_Complete.response. Note that the procedure also contemplates the possibility of the direct LTE-Advanced link being the most convenient one. In this case, the MN sends the MIH_MN_HO_Complete.request message directly to the BS.

\footnotetext{
${ }^{2}$ Note that the procedure would be the same in case of degradation of the LTE-Advanced link.
} 


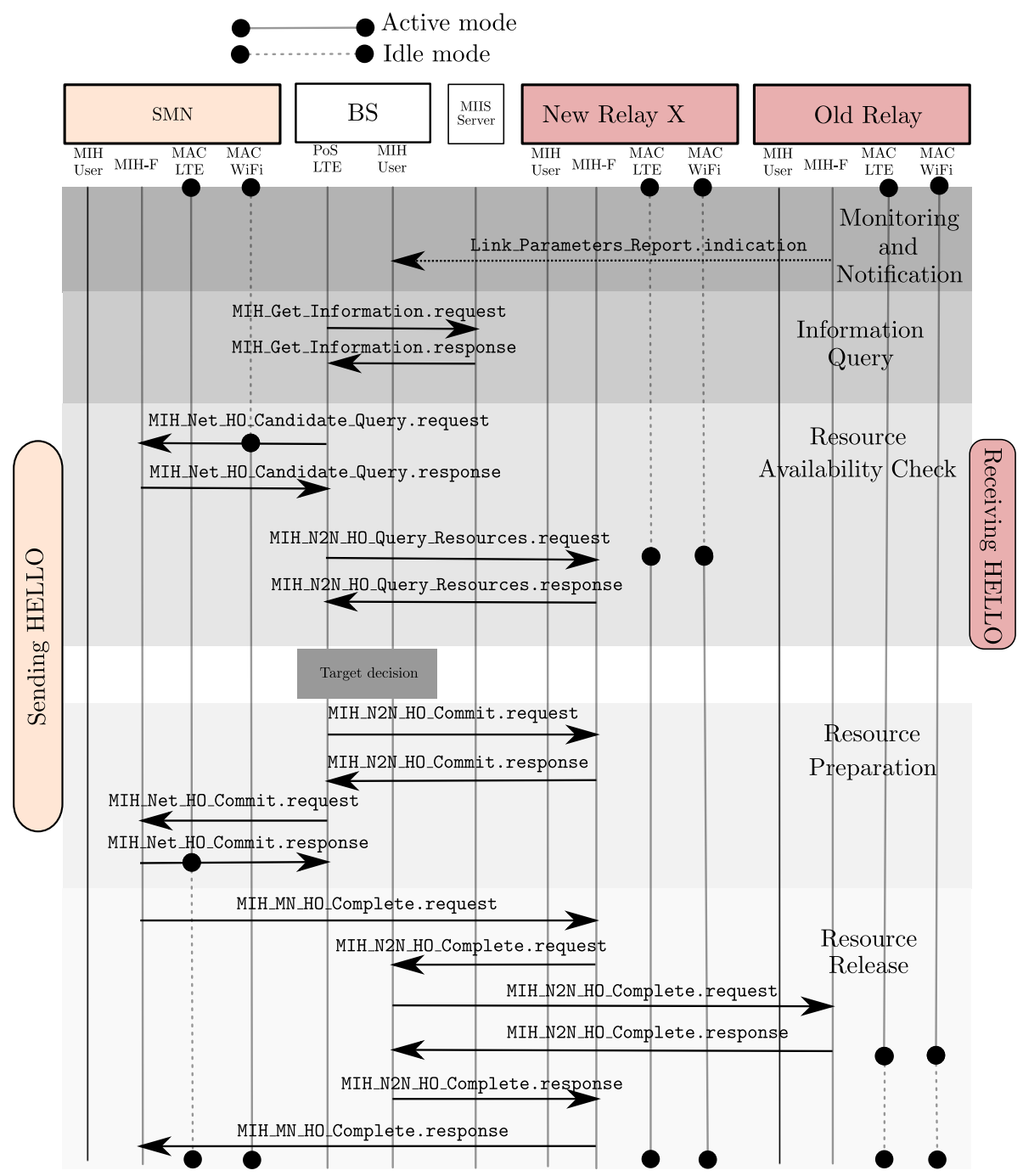

Figure 3.4: MIDRES procedure to change the MR. 


\subsubsection{Situation 3}

When the LTE-Advanced SINR level at the SMN is higher than the quality threshold, an event is sent from its lower layers to the MIH-User. This LinkUp event is notified by the SMN to the BS through a Link_Parameters_Report.indication message. The signaling procedure is the same as in Situation 2 with the possibility of not selecting any other MR.

\subsection{Assessment methodology and system mod- eling}

Assessment methodology in this work was based on system-level simulations. The baseline for the simulation method and system modeling are the guidelines provided by [46] for the evaluation of IMT-Advanced technologies. Nevertheless, some simplifications have been considered to reduce the complexity of the methodology proposed in [46].

A simple network layout was assumed with an isolated cell with a unique $\mathrm{BS}$ in its center that serves a circular area with radius $R$. A population of MNs is spatially uniformly distributed within the cell area. The initial position of a user $u$ is randomly taken in polar coordinates from two uniform distributions, being the radius, $r_{u}=\sqrt{U\left[0, R^{2}\right]}$ and the angle $\alpha_{u}=U[0,2 \pi]$.

Although in this model the cell layout deviates from the original one specified in [46], the same SINR Cumulative Distribution Function (CDF) as in the original layout is found in the cell of the new layout. This is achieved thanks to a position-SINR relation model specifically designed for this purpose. Using a complete simulator, with the original layout, the received SINR CDFs after antenna receiver were obtained for the different evaluation scenarios: Suburban Macrocell (SMa), Rural Macrocell (RMa), Urban Macrocell (UMa), Urban Microcell (UMi), and Indoor Hotspot (InH). In Section A.1.2, the distribution of these scenarios is illustrated. Then for each user, position has a one-to-one correspondence with the SINR value according to the following formula:

$$
S I N R_{u}=C D F^{-1}\left(1-\left(\frac{r_{u}}{R}\right)^{2}\right),
$$

where $C D F$ is the SINR CDF for any particular scenario, and $C D F^{-1}$ is its inverse. It is worth noting that, in this model, the higher SINR values are obtained for the lower distances to the BS.

Multipath fading is not emulated in this assessment. Then, SINR values represent wideband measures. In LTE-Advanced, channel capacity was obtained for each user translating the wideband SINR value to a throughput value 
through Look-Up Table (LUT) calculated in link-level simulations. Different LUT have been achieved for each evaluation scenario and MN multi-antenna configuration. Similar throughput vs wideband SINR curves are found in Section A.1.2 or in [52]. Both, the complete system-level simulator and the linklevel simulator were validated in the framework of the Wireless World Initiative New Radio + (WINNER+) [59] and METIS projects [38], being also used in the IMT-Advanced candidate evaluation carried out within ITU-R [60]. The design and implementation of both simulators are part of this Thesis. Concerning $\mathrm{WiFi}$, channel capacity was calculated as a function of the distance between transmitter and receiver following the model described in [61]. Section A.1.1 describes the channel model of a WLAN 802.11n link.

MNs are in constant motion throughout the simulations with a fixed speed and follow a model similar to the Random Waypoint Model [62]. The MN path comprises a sequence of movement intervals in which direction is fixed. In each movement interval, the starting point is the ending point of the previous interval, while the ending point is drawn from a spatially uniform distribution over the cell area.

MNs can be in two states and do not change this state during the simulation. MNs in the idle state can relay signals while MNs in the active state are receiving data and cannot relay signals. Concerning the traffic model, for the sake of simplicity a Constant Bit Rate (CBR) traffic source has been used. Regarding scheduling, LTE-Advanced performs a proportional fair allocation of resources [63].

Finally, concerning MIH signaling, it is worth noting that the simulation tool models the MIH messages and accounts for the overhead that this additional signaling entails. Only the MIH subscription messages that can be originated both locally and remotely have not been considered in the signaling burden. Concerning handover delay, the simulation takes into account the idle to connected mode latency in LTE-Advanced and all the time required in the discovery and connection phase of the WiFi interface.

Additional assumptions of the simulation methodology are indicated in Table 3.1 .

\subsection{Results and discussion}

MIDRES was designed to make MIH protocol compatible with the LTEAdvanced system. With this aim, it is necessary to perform an optimal threshold setting to find a trade-off between channel quality improvement and signaling overhead. Hence the importance of Section 3.5.1. On the other hand, $\mathrm{MIH}$ signaling can cause a performance hit as the number of candidate relay 
CHAPTER 3. NETWORK-LAYER SOLUTION: MIH FOR MOBILE RELAYING

\begin{tabular}{l|c}
\hline \hline Scenarios/Speed (km/h)/Radius (m) & $\begin{array}{c}\mathrm{InH} / 3 / 20 \mathrm{UMi} / 3 / 67 \\
\mathrm{UMa} / 30 / 167 \mathrm{RMa} / 120 / 577\end{array}$ \\
\hline Number of Active Nodes & 10 \\
\hline Number of Idle Nodes & 50 \\
\hline Packet Size (bytes) & 128 \\
\hline Application Rate (Mbps) & 100 \\
\hline TTT (ms) & 2.4 \\
\hline WiFi Carrier frequency $(\mathrm{GHz})$ & 20 \\
\hline WiFi Bandwidth (MHz) & 15 \\
\hline WiFi Transmission Power (dBm) & $1 \mathrm{x} 1$ \\
\hline WiFi Antenna Configuration & $1 \mathrm{x} 2$ \\
\hline LTE-Advanced Antenna Configuration & 10 \\
\hline LTE-Advanced Bandwidth $(\mathrm{MHz})$ & \\
\hline \hline
\end{tabular}

Table 3.1: Basic parameters used in the performance assessment.

nodes increases since signaling overhead increases too. Section 3.5.2 presents some results concerning the RDA algorithm that reduces the number of candidate relays and, consequently, the signaling overhead. Next, the effect of the MN speed on the performance of mobile relaying is evaluated in Section 3.5.3. Finally, Section 3.5.4 is devoted to the study of cost savings that result from using mobile relaying in LTE-Advanced.

\subsubsection{Threshold evaluation}

The study of the quality threshold set by the MIH-User is of paramount importance to reach an equilibrium between signaling load and system performance. There exist three different thresholds:

1. WiFi threshold $\left(T h_{W i F i}\right)$ : If the WiFi link power falls below this threshold, the MR replacement process starts.

2. LTE-Advanced threshold $\left(T h_{L T E}\right)$ : This threshold activates the search of a MR to support the connectivity.

3. LTE-Advanced reactivation threshold: If the LTE-Advanced SINR surpasses this threshold, the SMN could be back to the single-hop LTEAdvanced communication.

For the sake of simplicity, in this chapter $T h_{W i F i}$ is set to $-82 \mathrm{dBm}$ and the reactivation threshold equals $T h_{L T E}$. This section only focused on the 
optimization of the LTE-Advanced threshold based on, firstly, the minimization of the number of required relays and, secondly, the maximization of the CEUT.
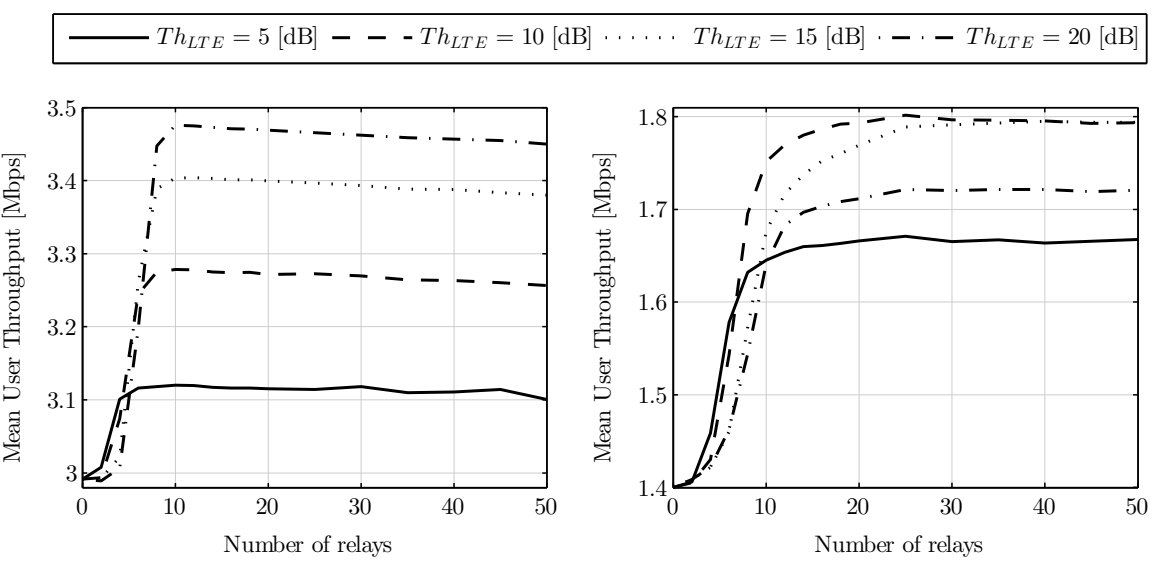

Figure 3.5: Threshold analysis for InH (left) and UMi (right) scenarios.

Figure 3.5 evaluates the Mean User Throughput (MUT) with an increasing number of MR available in the scenario $^{3}$. Two scenarios ( $\mathrm{InH}$ and UMi) and four thresholds are depicted. The number of active MN in the cell is 10, following the guidelines provided by [46]. Note that higher threshold implies that the user will be covered by a WiFi link with higher probability.

It can be observed that mobile relaying improves remarkably system performance. In the InH scenario, the higher the threshold is, the better the performance. It is always beneficial to use a MR since, in such a small situation, the $\mathrm{WiFi}$ interface exhibits higher data rates and reducing the hop length in LTE-Advanced also improves performance. However, there is an optimum in the number of MRs, which in the InH case is around 10. With more than ten MRs the additional diversity in the selection of the best MR does not compensate the increase in signaling. To sum up, for small scenarios the LTE-Advanced threshold must be set as high as possible, but the candidate set must be restricted to 10 .

However, for wider scenarios the selection is more challenging. As an example, this chapter analyzes the UMi case. If $T h_{L T E}$ is too small, the system does not benefit from the availability of relays. In fact, with a small number of available relays, it is better to choose a lower threshold since with higher probability the relay will not be beneficial for the link. On the contrary, if $T h_{L T E}$

\footnotetext{
${ }^{3}$ Note that $0 \mathrm{MR}$ represents the scenario without mobile relaying.
} 
CHAPTER 3. NETWORK-LAYER SOLUTION: MIH FOR MOBILE RELAYING

\begin{tabular}{l|c|c|c|c|c|c}
\hline \hline$T h_{L T E}[\mathrm{~dB}]$ & 10 & 11 & 12 & 13 & 14 & 15 \\
\hline CEUT $[\mathrm{Mbps}]$ & 1.5797 & 1.5916 & 1.5630 & 1.5524 & 1.5098 & 1.4377 \\
\hline \hline
\end{tabular}

Table 3.2: CEUT for different $T h_{L T E}$ in the UMi scenario.

is too high, with higher probability the WiFi link would be worse than the LTE-Advanced link and the system would waste resources on useless signaling. Therefore, an in-depth analysis is needed.

Figure 3.5 shows that the minimum number of relays to get good diversity is around 20. Note that this number is higher than in the $\mathrm{InH}$ case since more relays are required to have the same diversity in the selection of the best MR. On the other hand, Figure 3.6 shows the MUT CDF for this required minimum number of MRs. In the UMi scenario, the optimum threshold changes depending on the objective. To maximize the CEUT (5th percentile of the $\mathrm{CDF}$ ), the best threshold is between 10 and $15 \mathrm{~dB}$. In fact, the optimum is 11 $\mathrm{dB}$ as shown in Table 3.2.

$$
-T_{L T E}=5[d B]---T_{L T E}=10[d B] \cdots \cdots \cdot T_{L T E}=15[d B] \cdot-T_{L T E}=20[d B]
$$
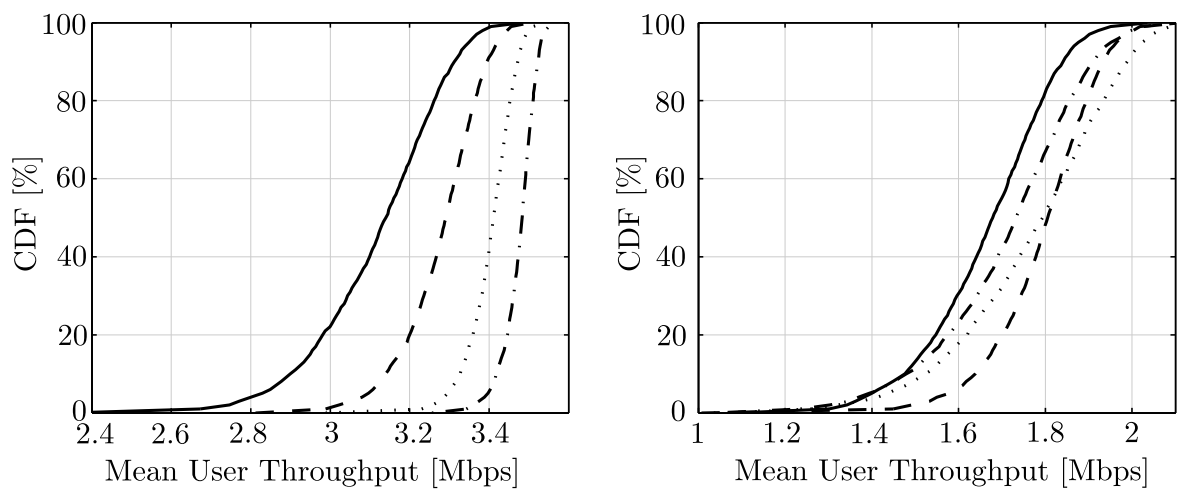

Figure 3.6: CDF of the mean user throughput for InH (left) and UMi (right) scenarios.

As a guideline, for the UMa scenario, the required minimum number of MRs is around 25, and the optimum threshold is $12 \mathrm{~dB}$. Concerning the RMa scenario, the number of relays is around 35 , and the optimum threshold is again $12 \mathrm{~dB}$. 


\subsubsection{Performance evaluation of RDA}

The RDA is useful for reducing the MIH signaling. Without the use of this algorithm by the MIIS server, the BS would send a MIH_N2N_HO_Query_Resources.request to all idle nodes. As the number of cooperative nodes increases the MIH protocol could be infeasible. Thus, the RDA algorithm only requires that the MIIS Server knows the MNs location. As discussed in Section 3.2.4, LTE-Advanced standard encompasses several positioning methods that can be used for this purpose.

In this section, a UMa scenario is assumed with $T h_{L T E}=12 \mathrm{~dB}$. Figure 3.7 shows the MUT varying the parameter $R_{R D A}$ for an increasing number of MRs. It can be seen that, when restricting the search area, a larger number of cooperative relay nodes is required to get the same MUT since the probability of a relay being in the search area is smaller. However, when the number of available relays increases, there is an increment in the signaling load that can be reduced with the RDA. In fact, the optimum value of $R_{R D A}$ depends on the number of available MRs as shown in Figure 3.7. For a higher number of cooperative nodes, a smaller search radius must be used since this reduces the signaling overhead.

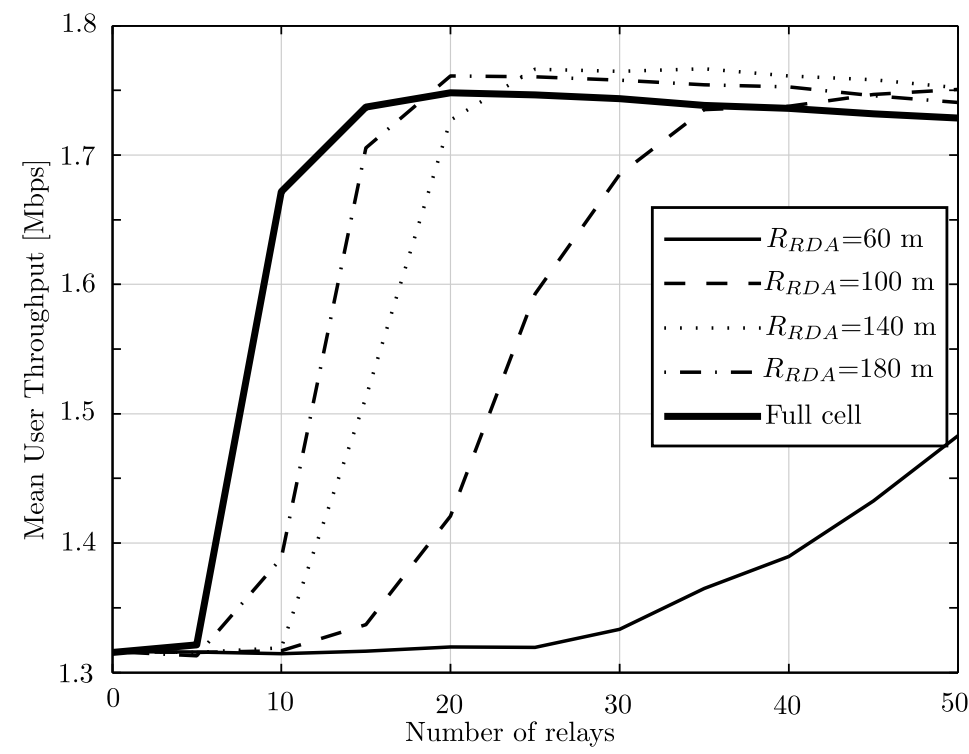

Figure 3.7: Mean user throughput for different $R_{R D A}$ values in a UMa scenario. 


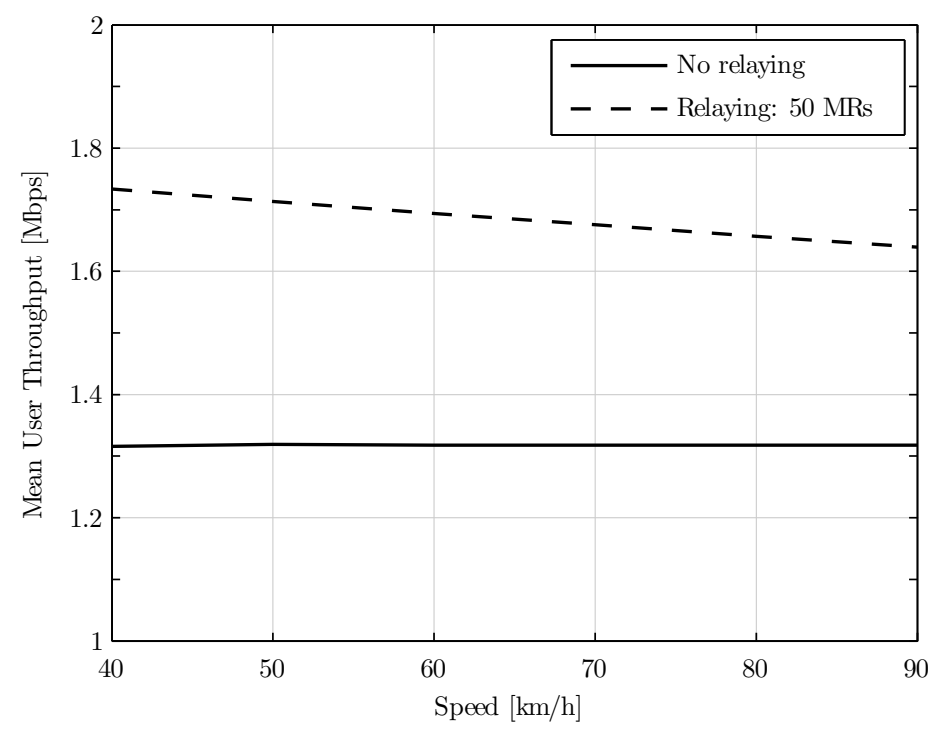

Figure 3.8: Mean user throughput for different values of MN speed in a UMa scenario.

\subsubsection{Velocity impact evaluation in performance}

This section evaluates the effect of the MN speed on the mobile relaying performance. A UMa scenario was simulated in which user speed augmented from 40 to $90 \mathrm{~km} / \mathrm{h}$. Figure 3.8 shows that the performance of mobile relaying deteriorates as the user speed increases because the mobile relaying links are less stable, and signaling and handover delay degrade the MUT. However, even with $90 \mathrm{~km} / \mathrm{h}$, mobile relaying is preferred as compared with the conventional single-hop scenario due to the fast-switching capacity of MIDRES and the good coverage of $\mathrm{WiFi}$ in outdoor.

\subsubsection{Deployment cost in an LTE-Advanced system}

LTE-Advanced systems are designed to increase end user data rates as compared with $3 \mathrm{G}$ systems. These high data rates may only be offered to users near the BS, creating a situation of unfairness between MNs. However, operators aim to offer higher rates to as many users as possible with the least possible cost. The use of MRs is considered as a key factor to increase the coverage thus deploying a lightweight wireless system. 


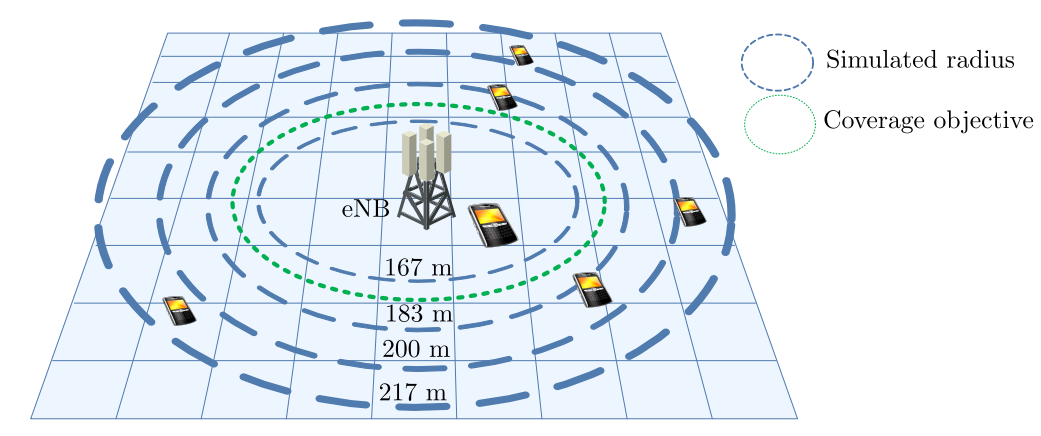

Figure 3.9: Simulation scenario to estimate the deployment cost of an LTE-Advanced system.

A simplified high-level financial analysis of the LTE-Advanced deployment was performed to assess the reduction of costs that can be achieved through the use of MRs. This study was carried out in a fictitious region of an area of 200 square kilometers similar to that described in [64], which represents a typical European city scenario. Although the total population was assumed to be 500,000 people, the LTE-Advanced network was expected to cover only $70 \%$ of the population. In particular, according to [64], the number of LTE-Advanced subscribers served by one operator in this fictitious scenario was predicted to be, at most, 55,000 by the third year. That is to say, a population density of 275 LTE-Advanced subscribers per $\mathrm{km}^{2}$ is considered.

To study the real economical impact of mobile relaying, a quality criterion for Cell-Edge User Spectral Efficiency (CEUSE) of $0.12 \mathrm{~b} / \mathrm{s} / \mathrm{Hz}$ was fixed for a UMa scenario. Multiple simulations were performed to assess the number of base stations required to achieve such quality criterion in a scenario with the population mentioned above density for different cell radius configurations with and without relaying. Figure 3.9 illustrates the simulation scenario of an LTE system with relays. While the blue circles represent the simulated radii, the green circles represent the coverage objective to achieve $0.12 \mathrm{~b} / \mathrm{s} / \mathrm{Hz}$. The received SINR CDF of the cells of 167, 183, 200, and $217 \mathrm{~m}$ are showed in Figure A.4 in Section A.1.2. For each cell radius configuration, the proper number of total users was calculated according to the fixed population density. The number of active MNs in each cell was $1 / 3$ of this total number, being the rest of users in the idle state. The number of possible MRs in each cell is a percentage of the number of idle users. The considered percentages range from $0 \%$ (network without relaying) to the $100 \%$ in steps of $20 \%$. In all simulations $T h_{L T E}=12 \mathrm{~dB}$. 


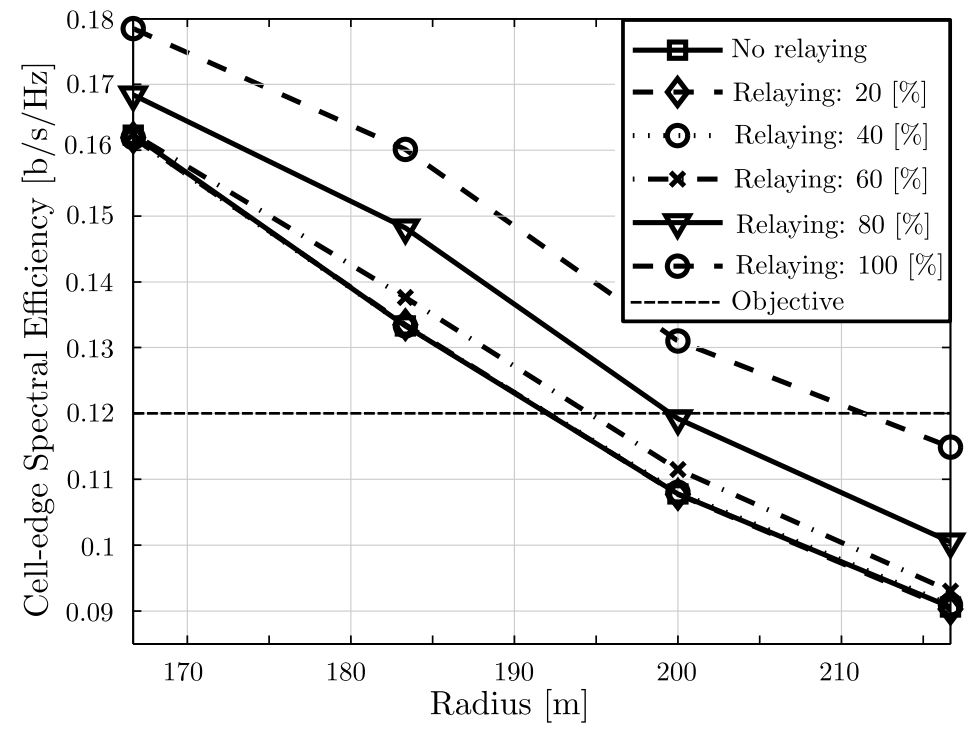

Figure 3.10: Cell-edge user throughput versus cell radius with different percentages of cooperative nodes.

As shown in Figure 3.10, a network without relays reaches the quality criterion with a cell radius of $192.35 \mathrm{~m}$, whereas with a percentage of $80 \%$ among idle nodes, coverage increases by $7.46 \mathrm{~m}$, which represents an increase of $7.91 \%$. With $100 \%$ of relay nodes, coverage increases $19.35 \mathrm{~m}$, that is, a $21.16 \%$. Note that without using relaying and until a percentage of $60 \%$ of idle nodes, the CEUSE is almost equal for different cell radius.

For the financial analysis, CAPital EXpenditures (CAPEX) and OPex EXpenditures (OPEX) per year were calculated. In the CAPEX analysis it was assumed that the mobile operator deploys LTE-Advanced using the available infrastructure. Therefore, civil work expenditures were not taken into account, neither the cost of acquiring spectrum licenses, since operators may use frequency bands of current technologies to minimize the cost of LTE-Advanced. Finally, CAPEX encompasses LTE-Advanced equipment cost (50 k€ per site [65]), labor-related deployment cost (10 $\mathrm{k} €$ per site) and upgrading the existing backhaul ( $2 \mathrm{k} €$ per site). The number of sites (composed of three cells in this study) multiplied by the investment cost per site yields the overall operator network investments. By combining the coverage area and the BS range, previously calculated, it is easy to obtain how many BSs are required. 
On the other hand, OPEX accounts for site rental (10 $\mathrm{k} €$ per site per year [65]), data backhaul costs (5 $\mathrm{k} €$ per site [65]), maintenance (3 $\mathrm{k} €$ per site [65]), marketing cost (1.85€ per person [66]), the subsidy paid by the operator to reduce the price of new mobile terminals, thus facilitating the migration of subscribers (160 € per subscriber [66]). Similarly, OPEX was calculated multiplying the number of sites by the running cost (site rental, data backhaul, and maintenance costs) per site plus marketing costs and terminal subsidies. In addition, this quantity is increased by administrative costs (addition of $10 \%$ [66]). Note that only one terminal replacement per user was supposed in this study. Moreover, OPEX is increased with the Annuity Payment (AP) of a loan for CAPEX requirements over a period of three years at an interest rate of $4 \%$ with the following formula:

$$
\mathrm{AP}=\operatorname{CAPEX} \frac{\left(1+\frac{p}{100}\right)^{N_{y}} \frac{p}{100}}{\left(1+\frac{p}{100}\right)^{N_{y}}-1},
$$

where $p$ and $N_{y}$ are the interest rate and the duration of the loan. Table 3.3 summarizes the above-mentioned costs of CAPEX and OPEX. Table 3.4 shows the resulting deployment costs. For instance, according to the simulations, CAPEX varies from around $43 \mathrm{M} €$ with a basic LTE system to around $36 \mathrm{M} €$ when mobile relaying is added. This represents a $17.47 \%$ saving.

\begin{tabular}{l||l|l}
\hline \hline \multirow{3}{*}{ CAPEX } & Equipment & $50 \mathrm{k} € /$ site \\
& Labor-related costs & $10 \mathrm{k} € /$ site \\
& Upgrading the existing backhaul & $2 \mathrm{k} € /$ site \\
\hline \multirow{4}{*}{ OPEX } & Site rental & $10 \mathrm{k} € /$ site/year \\
& Data backhaul & $5 \mathrm{k} € /$ site \\
& Maintenance & $3 \mathrm{k} € /$ site \\
& Marketing & $1.85 \mathrm{k} € /$ person \\
& Terminal subsidies & $160 € /$ subscriber \\
& Administrative costs & $10 \%$ of other running costs \\
& Loan for CAPEX & $\mathrm{AP}$ \\
\hline \hline
\end{tabular}

Table 3.3: Summary of infrastructure costs.

\subsection{Conclusions}

LTE has been designed as a future technology to cope with future user requirements. This chapter has proposed a new relay selection mechanism based 
CHAPTER 3. NETWORK-LAYER SOLUTION: MIH FOR MOBILE RELAYING

\begin{tabular}{l|c|c}
\hline \hline Scenario & CAPEX [M€] & OPEX [M€] \\
\hline LTE & 43.09 & 33.23 \\
\hline LTE-Advanced $(80 \%)$ & 39.93 & 31.07 \\
\hline LTE-Advanced $(100 \%)$ & 35.57 & 28.11 \\
\hline \hline
\end{tabular}

Table 3.4: Cost analysis of the LTE-Advanced deployment.

on the use of MIH signaling that includes mobile relaying as an additional technique in an LTE-Advanced system. The implementation of mobile relaying in LTE-Advanced certainly enhances the overall system performance. Besides, the proposed relay selection procedure is a low-cost solution because no modifications of the LTE-Advanced system architecture are required for its implementation.

However, the MIH-Driven Relay Selection Mechanism must be set up carefully, and several aspects have been discussed throughout the chapter. First, the SINR threshold that triggers the selection of relay must coincide with the point in which the cellular system is unable to cover user quality of service. Moreover, the selection of the relay entails additional signaling that must be reduced to a minimum to make the most of mobile relaying. A simple technique has been shown in this chapter to highlight the importance of this signaling overhead.

Finally, this chapter has shown the relevance of mobile relaying to reduce deployment costs. In a typical European city scenario, the reduction of CAPEX and OPEX is about $17.47 \%$ and $15.39 \%$ for $100 \%$ idle terminals acting as relays, respectively. 


\section{Chapter 4}

\section{Radio solution: non-coherent communications schemes}

\subsection{Introduction}

Mobile communication systems have been traditionally based on coherent reception, for which a set of pilot sequences is sent to the receiver to estimate the channel and perform coherent data detection. However, the use of this coherent communication has three main drawbacks, namely, pilot contamination, loss in spectral efficiency, and the increase of the pilot overhead with channel variability and number of transmit antennas.

Pilot pollution arises when a transmitter sends pilot sequences in the same resources as nearby transmitters, thus polluting the channel estimate of other receivers, and hence, reducing the system capacity. With respect to the loss in spectral efficiency, coherent systems must dedicate a significant portion of the available resources to pilot transmission to estimate the channel with sufficient accuracy [30]. This implies efficiency loss since these resources could have been used to transmit data instead. Furthermore, and related to the scalability problem, this efficiency loss depends directly on the number of transmit antennas and the channel variability. In particular, if pilot power can be adjusted, then the optimal number of training symbols is equal to the number of transmit antennas [30]. Otherwise, the number of training symbols should be increased even beyond this value [30]. Moreover, to maintain the same channel estimation accuracy for highly mobile channels, the amount of training must 


\section{CHAPTER 4. RADIO SOLUTION: NON-COHERENT COMMUNICATIONS SCHEMES}

be increased. In fact, to maximize the overall transmission rate, some studies claim that half of the coherence interval should be used to transmit pilots [69].

Although LTE pilot design aims at overcoming these drawbacks, the problems still prevail, especially in large-scale MIMO systems and high-speed scenarios. This fact has triggered recent efforts on the design and integration of non-coherent communication schemes, which do not send pilots to the receiver for decoding, and therefore, remove totally the drawbacks of coherent systems $[33,34]$. Of particular relevance is the activity developed in the METIS project [38], which corresponds to the work done in this Thesis.

In this chapter, non-coherent schemes are analyzed and compared with coherent schemes in realistic channel conditions of antenna correlation and time and frequency correlation. Particular attention is paid to the Grassmannian signaling ${ }^{1}$ scheme since this is the most promising technology investigated in this Thesis. After this comparison, this chapter deals with the detection of a multi-user MIMO transmission using Grassmannian signaling. The solution to this problem reinforces further the interest in the integration of this noncoherent scheme in the future 5G technology. This matter is the last subject addressed in this chapter. The rest of the chapter is organized as follows:

- Section 4.2 shortly describes the main drawbacks of coherent systems.

- Section 4.3 compares the performance of coherent and non-coherent signaling schemes over single-channel temporally-correlated scenarios.

- Section 4.4 proposes a concatenation technique that yields a spectrallyefficient time-frequency Grassmannian signaling scheme.

- Section 4.5 compares the performance of Grassmannian signaling with transmission diversity techniques of LTE over multiple-channel time and frequency-varying spatially correlated scenarios.

- Section 4.6 makes a proposal for the transmission and reception of a multi-user MIMO signal using Grassmannian-based non-coherent communications.

- Section 4.7 discusses about the integration of Grassmannian signaling for D2D and multi-hop communications. This section does not delve into the details; rather it aims at shedding light on the potential interest of this mechanism of transmission for the new $5 \mathrm{G}$.

- Section 4.8 outlines the main conclusions of this chapter.

\footnotetext{
${ }^{1}$ Throughout this chapter, the term signaling is equivalent to the term code.
} 


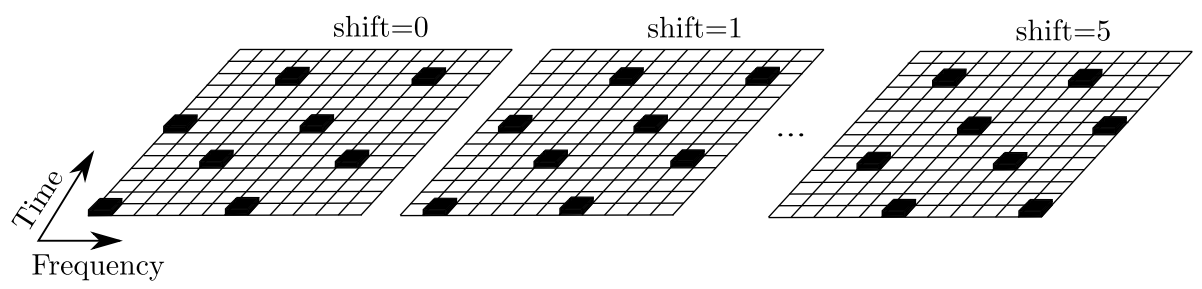

Figure 4.1: Shifts of the cell-specific RS (one antenna port).

\subsection{Pilot design in the LTE technology}

The 3GPP also discussed the three main drawbacks of coherent communications mentioned in the introduction during the design of the pilot sequences of LTE, also known in the 3GPP terminology as Reference Signals (RSs). Some of the solutions proposed in LTE are detailed in this section.

\subsubsection{Pilot contamination}

In the downlink, pilot contamination is partially removed in the cell-specific RS through the use of up to six frequency cyclic shifts of the pilot positions depicted in Figure 4.1. These shifts depend on the PCI such that each shift covers 84 PCIs from a total number of 504 PCIs. Therefore, a reuse of shifts is needed when the network is planned. For example, Figure 4.2 illustrates an example of the planning of the RS allocation. These cyclic shifts avoid the cross interference between pilots of six cells but causing interference to the data blocks of nearby cells. Additionally, this pilot arrangement causes interference from data blocks to pilots, polluting, therefore, the channel estimation. On the other hand, in the uplink, pilot contamination is partially removed with the use of orthogonal base sequences based on Zadoff-Chu sequences. The time-domain cyclic shift of these base sequences provides orthogonality between these signals. However, this orthogonality is not guaranteed at the receiver unless the length of the cyclic shifts is greater than the channel impulse response. Note that, for the uplink demodulation RS, only twelve cyclic shifts are defined, and hence, reuse of the pilot sequences is applied in the network planning. This reuse could still cause some pilot pollution, and this drawback could be aggravated when using MIMO spatial multiplexing since several pilot sequences should be transmitted from the same user equipment for adequate channel estimation. 


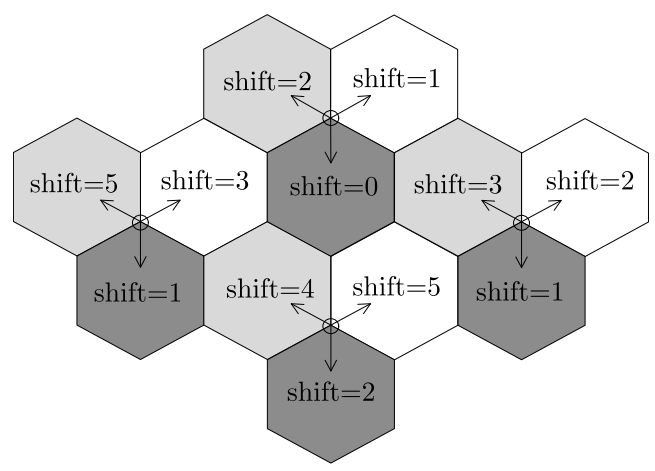

Figure 4.2: Example of planning of the RS transmission of a set of cells.

\subsubsection{Loss of spectral efficiency and scalability problem}

Mobile communication standards are usually designed to work well under highspeed conditions. However, this scenario requires the use of a significant amount of resources for the pilot transmission, and hence, a substantial degradation of the user plane data rates is experienced. Thus, a trade-off exists between high accuracy in the channel estimation and availability of resources for data transmission. In particular, in LTE, for two transmit antennas, about $10 \%$ of resources are reserved for pilots, maintaining good performance even for high-speed users. For four antennas, $20 \%$ of the resources should be reserved to maintain the same channel estimation accuracy. However, only around $15 \%$ of resources carry pilots. As a result, the channel estimation accuracy for four transmit antennas is reduced since there is less time-domain pilot density for the third and fourth antennas. This pilot arrangement assumes that the transmission modes with four transmit antennas will be only used for low-speed users, which is an important limitation to provide mobile broadband services in vehicular environments with a high number of transmit antennas.

\subsection{Comparison of coherent and non-coherent schemes: single-channel MIMO systems}

\subsubsection{System model}

We consider a single-user link with $M$ transmit antennas and $N$ receive antennas $(M \times N$ MIMO system). The transmitter uses a space-time modulation 


\subsection{Comparison of coherent and non-coherent schemes: single-channel MIMO systems}

to send information blocks of $K$ bits over $T$ channel uses ${ }^{2}$ and $M$ transmit antennas. The transmission rate in bits per channel use (bpcu) is $R=K / T$. Each block consists of a $T \times M$ complex matrix

$$
\mathbf{X}=\left[\mathbf{x}_{1}, \mathbf{x}_{2}, \ldots, \mathbf{x}_{t}, \ldots, \mathbf{x}_{T}\right]^{\top},
$$

where $\mathbf{x}_{t} \in \mathbb{C}^{M \times 1}, t \in\{1, \ldots, T\}$, is the signal transmitted by the $M$ antennas at channel use $t$, and the superscript $(\cdot)^{\top}$ stands for matrix transposition operation. After $T$ channel uses, the receiver processes the $T \times N$ matrix $\mathbf{Y}=\left[\mathbf{y}_{1}, \mathbf{y}_{2}, \ldots, \mathbf{y}_{t}, \ldots, \mathbf{y}_{T}\right]^{\top}$, where

$$
\mathbf{y}_{t}^{\top}=\mathbf{x}_{t}^{\top} \mathbf{H}_{t}+\sqrt{\frac{M}{\rho T}} \mathbf{z}_{t}^{\top}
$$

is the complex vector received at channel use $t, \mathbf{z}_{t} \in \mathbb{C}^{N \times 1}$ is the Additive White Gaussian Noise (AWGN) with zero-mean and unit-variance, $\rho$ is the SNR (see Appendix $\mathrm{C}$ for more details about the SNR calculation), and $\mathbf{H}_{t} \in \mathbb{C}^{M \times N}$ is the channel matrix between the transmit and receive antennas.

We assume that the channel is temporally-correlated through a sum-ofsinusoids statistical model, which is an improved version of the original Jakes' model [70]. In this model, the correlation between two samples separated by $T_{s}$ seconds is $J_{0}\left(2 \pi f_{d} T_{s}\right)$. Here, $J_{0}$ is the zeroth-order Bessel function of first kind, $f_{d}=v f_{c} / c$ is the Doppler frequency, $v$ is the relative speed between the transmitter and the receiver, $f_{c}$ is the carrier frequency of the signal, and the constant $c=3 \cdot 10^{8} \mathrm{~m} / \mathrm{s}$ is the speed of light.

In this work, two different types of detectors are considered at the receiver side, namely the coherent detector, which has availability of the channel coefficients for the detection, and the non-coherent one, which works without any knowledge of the channel. Figures 4.3 (a) and (b) show the block diagram of the transmitter and receiver of the coherent and non-coherent schemes, respectively. It can be observed that the coherent setup includes a channel estimation stage to acquire the channel coefficients, as it will be next elaborated. These two MIMO schemes are deeply analyzed in Section 4.3.2.

\subsubsection{Non-coherent schemes: DUSTM}

DUSTM is an extension of the DPSK modulation to support MIMO communications [71]. As in every differentially-encoded constellation, each transmitted signal in this scheme is a reference for the next one. This scheme is intended for slow-varying channels, where the channel can be assumed approximately

\footnotetext{
${ }^{2}$ Throughout this Thesis the terms channel use and time slot are interchangeable.
} 


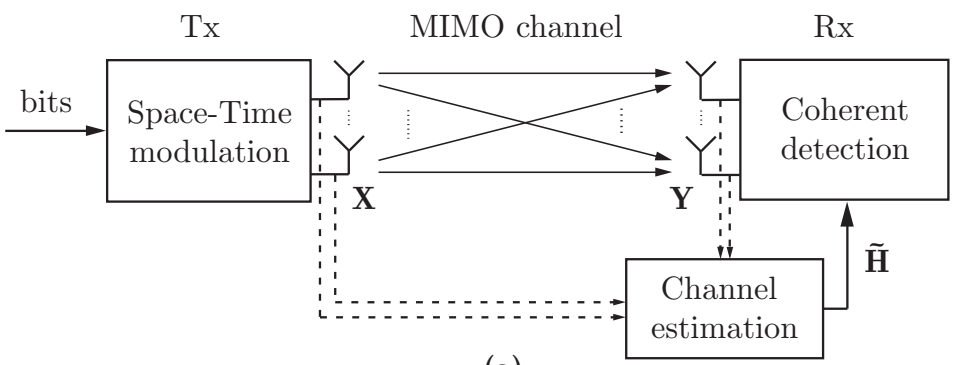

(a)

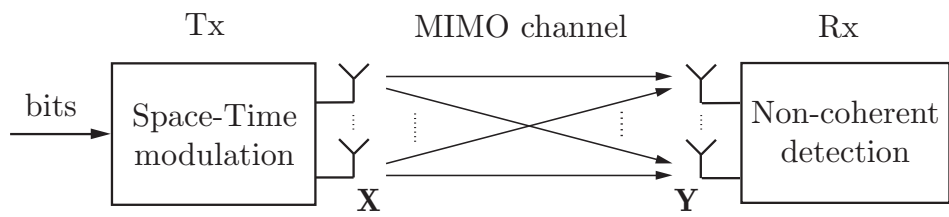

(b)

Figure 4.3: Block diagram of the evaluated MIMO system. (a) With coherent detection, (b) with non-coherent detection.

constant during any $2 M$ consecutive channel uses. However, for fast-fading channels, this assumption is not valid any longer, and DUSTM deteriorates as the normalized Doppler frequency increases.

\section{Transmitter}

The codebook of symbols is composed of a set of $M \times M$ unitary matrices, i.e., $T=M$ in this coding scheme. The signal matrix to be transmitted is differentially encoded from the matrix transmitted in the previous block, denoted by $\overline{\mathbf{X}}$, as

$$
\mathbf{X}=\mathbf{V}_{l} \overline{\mathbf{X}}
$$

Here, $\mathbf{V}_{l}, l=\{0,1, \ldots, L-1\}$, belongs to a codebook $\mathbf{V}$ of $L=2^{R M} M \times M$ unitary diagonal matrices [71]. The set of $\mathbf{V}$ matrices can be constructed in a cyclic way starting from $\mathbf{V}_{1}$, which gives the first element of the codebook as

$$
\mathbf{V}_{1}=\left[\begin{array}{ccc}
e^{\frac{j 2 \pi u_{1}}{L}} & \cdots & 0 \\
0 & \ddots & 0 \\
0 & \cdots & e^{\frac{j 2 \pi u_{M}}{L}}
\end{array}\right], u_{m} \in\{0,1, \cdots, L-1\}, m=1,2, \cdots, M
$$




\subsection{Comparison of coherent and non-coherent schemes: single-channel MIMO systems}

The rest of elements are subsequently obtained as $\mathbf{V}_{l}=\left(\mathbf{V}_{1}\right)^{l}, l=$ $\{0,1, \cdots, L-1\}$. The performance of this codebook is significantly degraded for $R>2$ [71]. This result will be verified by simulations in Section 4.5.2. To initialize the communication, the original $\overline{\mathbf{X}}$ is considered as an $M \times M$ identity matrix.

\section{Receiver}

With the approximation that the channel is constant during $2 M$ time slots, i.e., $\mathbf{H}_{t} \approx \mathbf{H}_{t-1} \approx \mathbf{H}$, the received signals in two consecutive blocks are

$$
\begin{aligned}
& \overline{\mathbf{Y}}=\overline{\mathbf{X}} \mathbf{H}+\sqrt{\frac{M}{\rho T}} \overline{\mathbf{Z}}, \\
& \mathbf{Y}=\mathbf{X} \mathbf{H}+\sqrt{\frac{M}{\rho T}} \mathbf{Z},
\end{aligned}
$$

where $\mathbf{Z}=\left[\mathbf{z}_{1}, \mathbf{z}_{2}, \ldots, \mathbf{z}_{t}, \ldots, \mathbf{z}_{T}\right]^{\top}$ is the $T \times N$ noise matrix, and $\overline{\mathbf{Z}}$ denotes the noise matrix of the previous transmission. Substituting (4.2) into (4.4) and combining with (4.3), we obtain

$$
\mathbf{Y}=\mathbf{V}_{l} \overline{\mathbf{Y}}+\sqrt{\frac{M}{\rho T}}\left(\mathbf{Z}-\mathbf{V}_{l} \overline{\mathbf{Z}}\right)
$$

which is the fundamental differential receiver equation. Note that although, here, the desired signal is corrupted by noise with twice the variance, the channel matrix is not longer necessary for the detection stage. This results in the well-known $3 \mathrm{~dB}$ performance loss in effective SNR when the channel is unknown in comparison when it is known. The ML detection rule is directly

$$
\hat{\mathbf{V}}=\arg \min _{l}\left\|\mathbf{Y}-\mathbf{V}_{l} \overline{\mathbf{Y}}\right\|^{2}
$$

where the notation $\hat{\mathbf{V}}$ is used for the detected codeword.

\subsubsection{Non-coherent schemes: Grassmannian codes}

\section{Transmitter}

A Grassmannian codebook consists of a set of codewords $\mathbf{S}_{l}, l=\{0,1, \ldots, L-1\}$, with $L=2^{R T}$ [33] [34]. The Grassmannian codewords are classified under the category of USTM schemes, which are optimal for block-fading Rayleigh 


\section{CHAPTER 4. RADIO SOLUTION: NON-COHERENT}

COMMUNICATIONS SCHEMES

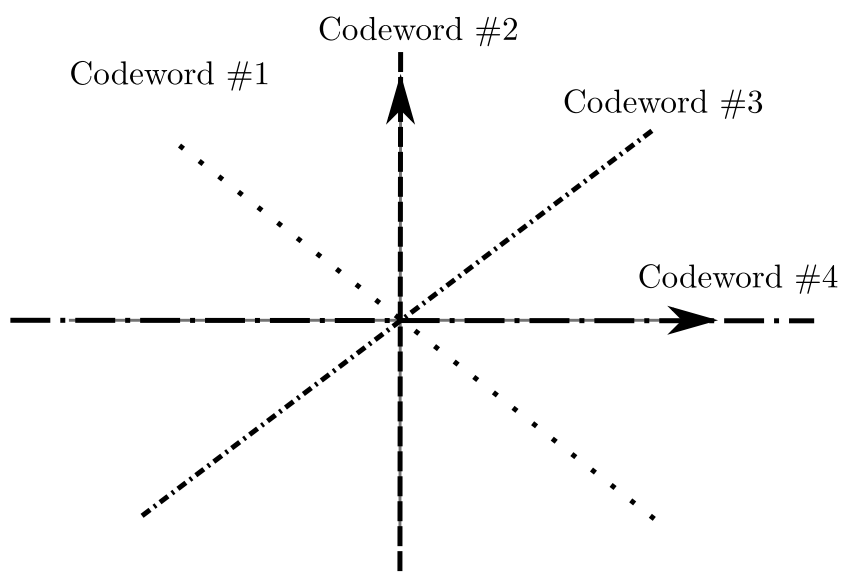

Figure 4.4: Exemplary Grassmannian codebook for $M=1$ antenna, $T=2$ time slots: 4 different directions in a plane.

channels of length $T$. These channels assume that channel coefficients are invariant for the duration of a block of $T$ channel uses and assume independent realizations in the next block duration. Several design methods for Grassmannian constellations of isotropically distributed unitary matrices can be found in [34], where the design criteria are based on selecting distant subspaces to minimize the error probability. The $M$ column vectors of each codeword represent the basis of an $M$-dimensional subspace that lies inside a $T$-dimensional space. At high SNR, when the input signal matrix $\mathbf{X}$, drawn from the Grassmannian codebook, is passed through a complex MIMO channel, the column vectors that span the original $M$-dimensional subspace are rotated and scaled, but they remain within the same subspace. Therefore, the transmitter has only to map the transmitted codewords to separate subspaces, represented by the Grassmannian codewords. Figure 4.4 shows an exemplary Grassmannian constellation composed of four different directions in a plane, which can be represented by four $2 \times 1$ matrices, i.e. four one-dimensional subspaces in a two-dimensional space.

\section{Receiver}

The particular subspace basis rotation is not detectable by a receiver without channel knowledge. However, the $M$-dimensional linear subspace spanned by this basis can be detected by using a Generalized Likelihood Receiver Test (GLRT) [72]. The GLRT criterion projects the received signal on the different 


\subsection{Comparison of coherent and non-coherent schemes:} single-channel MIMO systems

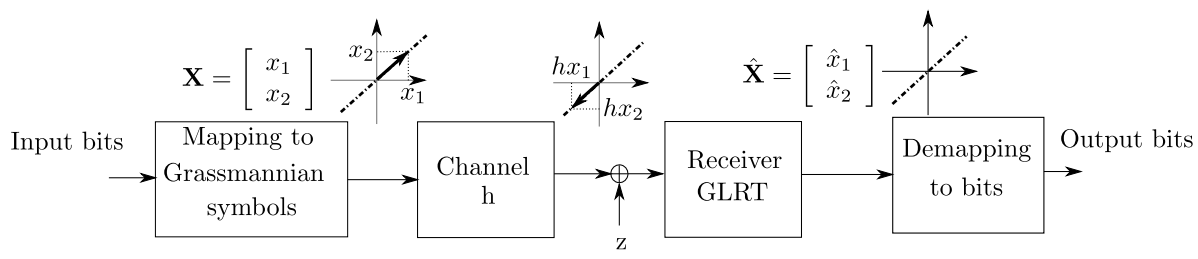

Figure 4.5: Block diagram of a non-coherent transceiver with $M=1$ antenna and $T=2$ time slots.

subspaces that compose the Grassmannian constellation. Then, it calculates the energies of all the projections and selects the projection that maximizes the energy as follows:

$$
\hat{\mathbf{X}}=\arg \max _{l} \operatorname{Tr}\left(\mathbf{Y}^{\mathrm{H}} \mathbf{S}_{l} \mathbf{S}_{l}^{\mathrm{H}} \mathbf{Y}\right)
$$

From the perspective of average symbol error probability minimization, in general, the GLRT provides a suboptimal result compared to the ML criterion. However, for the case of unitary constellations assumed in this work, GLRT offers the same performance as ML detection [72].

An exemplary procedure for transmission and detection of Grassmannian constellation during $T$ time slots is described next. Figure 4.5 shows the block diagram of the associated non-coherent transceiver, which uses $M=1$ antenna, $T=2$ time slots, and the Grassmannian constellation in Figure 4.4. First of all, the information bits to be transmitted, are mapped to Codeword \#3 through the matrix $\mathbf{X}$ (see Figure 4.4). After the codeword is transmitted, its underlying basis (the dark arrow in the subspace) is transformed by the channel, but it remains in the same subspace. Note that, in this example, the channel $h$ is the same for the two time slots. Although the non-coherent receiver cannot detect the particular transformation caused by the channel, at high SNR, it can indeed identify the subspace spanned by this basis. Therefore, the transmitted information can be recovered without any knowledge of the channel at the receiver.

\subsubsection{Coherent training-based schemes}

\section{Transmitter}

The well-known coding scheme proposed by Alamouti is the simplest fulldiversity OSTBC for the two transmit antenna case. In this scheme, $T=M=2$ 
and the $2 \times 2$ transmission matrix is structured as follows [28]:

$$
\mathbf{X}=\left[\begin{array}{cc}
s_{1} & s_{2} \\
-s_{2}^{*} & s_{1}^{*}
\end{array}\right]
$$

where the symbols $s_{i}, i=1,2$, are taken from a Quadrature Amplitude Modulation $(\mathrm{QAM})$ constellation $\Omega$ of size $|\Omega|$ and hence carry $\log _{2}(|\Omega|)$ code bits each.

For the $M=4$ case, we will include the performance of an OSTBC of rate $1 / 2$ within the comparisons, which is transmitted using $T=8$ channel uses. Its code matrix, defined in [29], is

$$
\mathbf{X}=\left[\begin{array}{rrrrrrrr}
s_{1} & -s_{2}^{*} & -s_{3}^{*} & -s_{4} & s_{1}^{*} & s_{2}^{*} & s_{3}^{*} & s_{4}^{*} \\
s_{2} & s_{1}^{*} & s_{4}^{*} & -s_{3} & -s_{2}^{*} & s_{1}^{*} & -s_{4}^{*} & s_{3}^{*} \\
s_{3} & -s_{4}^{*} & s_{1}^{*} & s_{2} & -s_{3}^{*} & s_{4}^{*} & s_{1}^{*} & -s_{2}^{*} \\
s_{4} & s_{3}^{*} & -s_{2}^{*} & s_{1} & -s_{4}^{*} & -s_{3}^{*} & s_{2}^{*} & s_{1}^{*}
\end{array}\right]^{\top}
$$

Another alternative that will be evaluated is the use of a QOSTBC [73], which needs $T=4$ channel uses for its transmission and has the following code matrix:

$$
\mathbf{X}=\left[\begin{array}{rrrr}
s_{1} & -s_{2}^{*} & -s_{3}^{*} & s_{4} \\
s_{2} & s_{1}^{*} & -s_{4}^{*} & -s_{3} \\
s_{3} & -s_{4}^{*} & s_{1}^{*} & -s_{2} \\
s_{4} & s_{3}^{*} & s_{2}^{*} & s_{1}
\end{array}\right]^{\top}
$$

In the $2 \times 2 \mathrm{MIMO}$ system performance evaluation, we will also include the Golden code for two antennas with $T=M$, which is a full-rate and full-rank STBC with the following code matrix [74]:

$$
\mathbf{X}=\frac{1}{\sqrt{5}}\left[\begin{array}{cc}
\alpha\left(s_{1}+s_{2} \theta\right) & \alpha\left(s_{3}+s_{4} \theta\right) \\
i \gamma\left(s_{3}+s_{4} \bar{\theta}\right) & \gamma\left(s_{1}+s_{2} \bar{\theta}\right)
\end{array}\right],
$$

where $\theta=\frac{1+\sqrt{5}}{2}$ is the Golden number, $\gamma=1+i \theta$ with $i=\sqrt{-1}, \bar{\theta}=1-\theta$, and $\alpha=1+i \theta$.

\section{Receiver}

The ML-decoding metric to be minimized over all possible values of codewords $\mathbf{X}$ for the Alamouti, rate-1/2 OSTBC, QOSTBC, and Golden codes is given by

$$
\hat{\mathbf{X}}=\arg \min _{\mathbf{X}}\|\mathbf{Y}-\mathbf{X} \widetilde{\mathbf{H}}\|^{2},
$$




\subsection{Comparison of coherent and non-coherent schemes: single-channel MIMO systems}

where $\widetilde{\mathbf{H}}$ is an estimate of the channel matrix. Here we assume a trainingbased scheme where an $M \times M$ matrix $\mathbf{P}$ containing training symbols is used to acquire the channel coefficients at the receiver side via Minimum Mean Square Error (MMSE) channel estimation [30], that is,

$$
\widetilde{\mathbf{H}}=\sqrt{\frac{M}{\rho}}\left(\frac{\rho}{M} \mathbf{I}_{M}+\mathbf{P}^{\mathrm{H}} \mathbf{P}\right)^{-1} \mathbf{P}^{\mathrm{H}} \mathbf{Y}_{\mathrm{P}},
$$

where $\rho$ and $\mathbf{P}$ are known a priori by the receiver, and $\mathbf{Y}_{\mathbf{P}}$ denotes the signal matrix received after the transmission of pilots.

\subsubsection{Performance analysis over time-varying channels}

\section{Simulation environment}

The aim of this section is to compare the performance of coherent and noncoherent open-loop communication schemes under realistic assumptions of channel variability. To evaluate the behavior of the different schemes with the transmission rate, we consider two representative values of this rate for the $2 \times 2$ system, particularly $R=2 \mathrm{bpcu}$ and $R=3 \mathrm{bpcu}$, at an SNR value of $25 \mathrm{~dB}$. Moreover, to see the effect of increasing the number of antennas, we will also evaluate a $4 \times 4$ system for the $R=2$ bpcu case and the same SNR value. The simulated coherent schemes for two transmit antennas and two channel uses are the well-known Alamouti and the Golden code schemes [28] [74]. For the $M=4$ case, we include the performance of an OSTBC of rate $1 / 2$ within the comparisons, which is transmitted using eight channel uses [29]. Another alternative that will be evaluated is the use of a QOSTBC [73], which needs four channel uses for its transmission. For all schemes, an ML receiver will be used. Only coherent schemes use an MMSE channel estimator.

Figure 4.6 shows the transmission configurations of coherent and noncoherent schemes analyzed in this section. Unlike the coherent schemes, Grassmannian signaling does not need any prior transmission of pilots, and their design rule assumes a block-fading channel of duration $T$ channel uses. Although the temporally-correlated channel does not match this feature, we will evaluate designs considering $T=4, T=6$, and $T=8$ for the $2 \times 2$ case, and, $T=8$ and $T=12$ for the $4 \times 4$ case to see the degradation of assuming large block lengths under temporal correlation. Concerning DUSTM, there is a single transmission of an $T \times M$ pilot matrix at the beginning of the communication, but its overhead can be disregarded assuming a high number of transmission blocks. On the other hand, coherent schemes will take into account the transmission of pilots during $33 \%$ of time. Note that data is transmitted into two blocks of length $T$ carrying $3 R T / 2$ bits to compensate the pilot overhead. To 


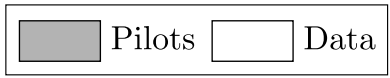

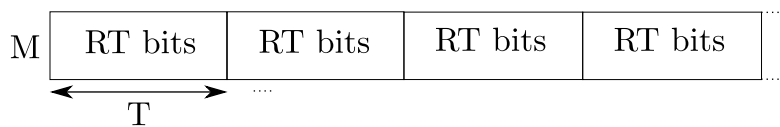

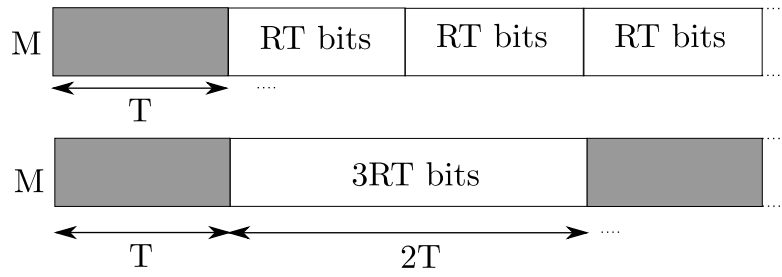

Grassmannian constellations

DUSTM

Coherent schemes

Figure 4.6: Transmission configurations for the coherent and non-coherent schemes under evaluation.

Table 4.1: Constellations selected for the coherent STC schemes.

\begin{tabular}{|c||c|c||c|c||c||c|}
\hline STC scheme & \multicolumn{2}{|c|}{ Alamouti } & \multicolumn{2}{|c||}{ Golden } & QOSTBC & OSTBC \\
\hline Rate (bpcu) & 2 & 3 & 2 & 3 & 2 & 2 \\
\hline x-QAM & 8 & $2^{4.5}$ & $2^{1.5}$ & $2^{2.25}$ & 8 & 64 \\
\hline
\end{tabular}

match the actual transmitted rate in bpcu, and hence be comparable with the non-coherent schemes, the constellations underlying the coherent codes have been carefully chosen as detailed in Table 4.1 .

\section{Results}

We evaluate the performance of the systems mentioned above according to FER versus normalized Doppler frequency, i.e., $f_{d} T_{s}$, where $T_{s}$ is the inverse of the coherence bandwidth. A frame corresponds to 24 channel uses. This number was chosen as the minimum common multiplier of all the $T$ values considered in this Thesis.

In Figure 4.7 and Figure 4.8, we compare the coherent and non-coherent schemes for $M=2$ and a transmission rate of 2 and 3 bits per channel use, respectively. The non-coherent schemes outperform the coherent schemes for low to medium values of $f_{d} T_{s}$. The main reason is that channel estimation errors strongly penalize the training-based schemes. For $R=2 \mathrm{bpcu}$, it is observed in Figure 4.7 that DUSTM outperforms the rest of transmission systems. In fact, 


\subsection{Comparison of coherent and non-coherent schemes: single-channel MIMO systems}

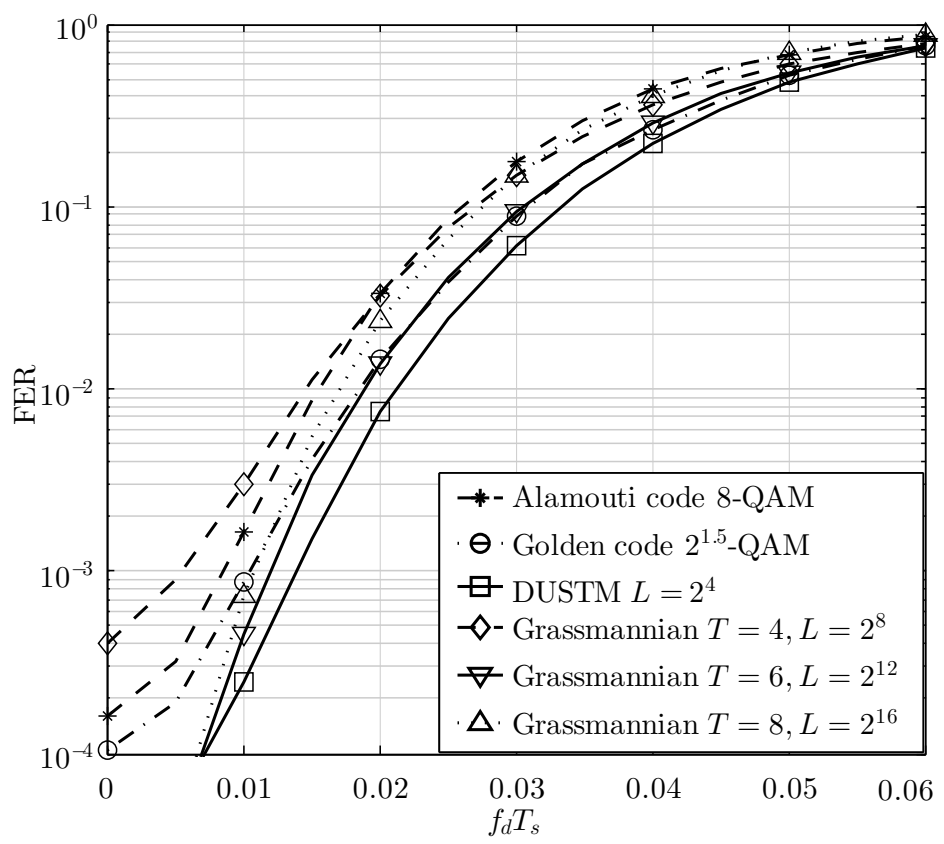

Figure 4.7: Performance comparison among coherent and non-coherent schemes with $M=2$ and $R=2$ assuming a temporally-correlated channel with different values of $f_{d} T_{s}$.

DUSTM shows high robustness to mobility for low data rates, even for nonstationary channels. In Figure 4.8, however, DUSTM suffers a performance loss about the Golden code and Grassmannian schemes as the product $f_{d} T_{s}$ increases. For $R=3 \mathrm{bpcu}$, Grassmannian signaling is the best-performing scheme since it outperforms both the DUSTM scheme and the coherent techniques for low to medium values of the product $f_{d} T_{s}$. However, note that at high normalized Doppler frequency values, the Grassmannian signaling and the Golden code attain nearly the same FER values.

For Grassmannian signaling, increasing the block length is, in general, positive to augment capacity, as can be seen when comparing $T=4$ and $T=6$ cases. However, Grassmannian requires the channel to be constant during the block and is, therefore, sensitive to mobility and channel variability. In fact, a block length of $T=8$ offers worse performance than $T=6$. This result suggests that an optimum block length can be found in time-selective channels. Therefore, we can conclude that in the temporally-correlated channel, having 


\section{CHAPTER 4. RADIO SOLUTION: NON-COHERENT COMMUNICATIONS SCHEMES}

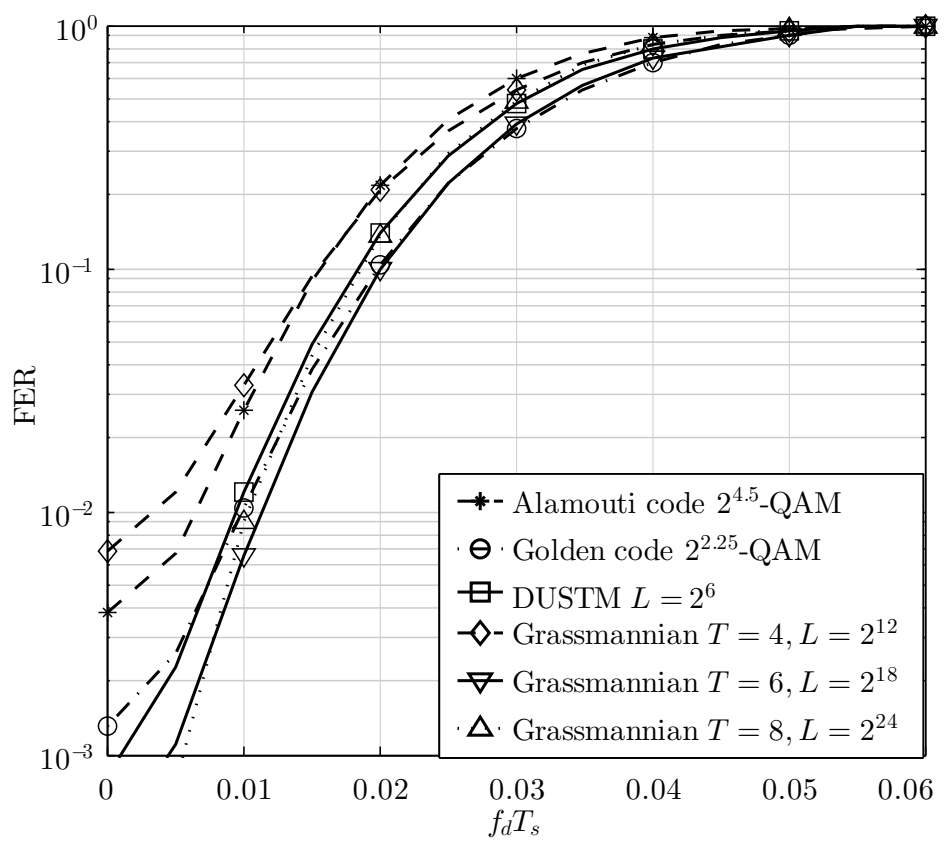

Figure 4.8: Performance comparison among coherent and non-coherent schemes with $M=2$ and $R=3$ assuming a temporally-correlated channel with different values of $f_{d} T_{s}$.

a Grassmannian code with a longer block length does not imply necessarily better performance, since the channel variations caused by mobility destroy the block-fading condition of the channel. This phenomenon is observed for both the $R=2 \mathrm{bpcu}$ and $R=3 \mathrm{bpcu}$ cases. It is important to emphasize that this fact is not satisfied when the normalized Doppler frequency is approaching zero since, in that case, it is well-known that the performance increases as $T$ grows [75].

In Figure 4.9, we compare again coherent and non-coherent schemes for $M=4$ transmit antennas. We can see that non-coherent schemes perform much better than the coherent schemes for all the evaluated normalized Doppler frequency values. This result motivates its relevance in the design of new openloop transmission techniques valid for higher-order MIMO configurations. In fact, the rate-1/2 OSTBC suffers a much higher performance loss than the rest of schemes. Unlike what is observed in Figure 4.7 for $R=2$ and a $2 \times 2$ MIMO configuration, the DUSTM scheme is no longer the best-performing 


\subsection{Comparison of coherent and non-coherent schemes: single-channel MIMO systems}

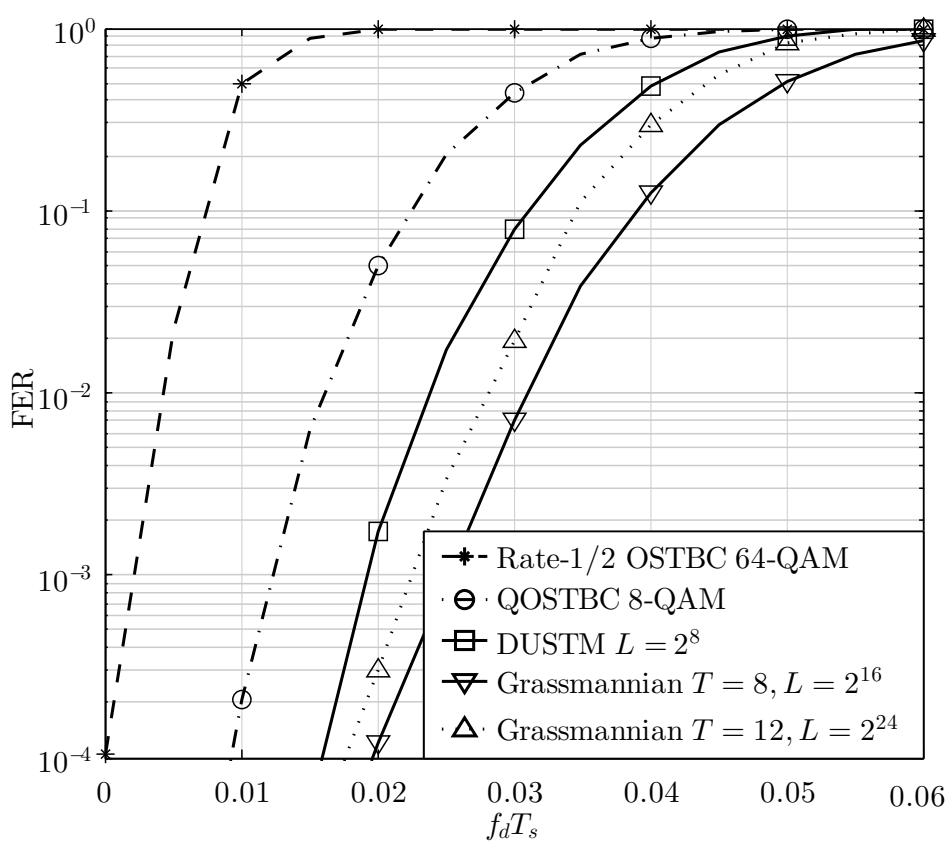

Figure 4.9: Performance comparison among coherent and non-coherent schemes assuming a temporally-correlated channel with different values of $f_{d} T_{s}$ for $R=2, M=4$.

scheme in a $4 \times 4$ MIMO system. Nevertheless, DUSTM still outperforms the coherent setups. The Grassmannian code with $T=8$ outperforms all schemes with a significant advantage. As with the schemes with $M=2$, increasing the Grassmannian block-length does imply better performance. In fact, having a Grassmannian code higher than $T=8$ channel uses causes a performance loss due to mobility.

Note that, in the analysis of Figures 4.7, 4.8 and 4.9, the relative speed between the transmitter and the receiver, the carrier frequency and the scenario under study are hidden through the normalized Doppler frequency. As an example of performance analysis at speed level, we show in Table 4.2 the maximum speed to achieve a FER of $10^{-2}$ for the best analyzed coherent and noncoherent schemes. From this analysis, we observe that non-coherent schemes offer better performance than the training-based schemes for high delay spread and high carrier frequency. It is important to emphasize that both parameters cannot be maximized at the same time since the delay spread is inversely 


\section{CHAPTER 4. RADIO SOLUTION: NON-COHERENT COMMUNICATIONS SCHEMES}

proportional to the carrier frequency. Thus, we analyzed a scenario in a dense urban environment with a delay spread of $10 \mu$ s operating at a carrier frequency of $2 \mathrm{GHz}$. Table 4.2 shows that, while coherent and non-coherent schemes offer a similar maximum speed for two antennas, Grassmannian codes are promising techniques for four antennas. In particular, the maximum sustained speed by the non-coherent schemes almost doubles that of the coherent schemes, reaching up to $165 \mathrm{~km} / \mathrm{h}$. This result motivates the interest in focusing non-coherent schemes in vehicular communications where a high number of antennas can be placed on the vehicle.

Table 4.2: Maximum speed in $\mathrm{km} / \mathrm{h}$ for the coherent and non-coherent schemes in an urban scenario.

\begin{tabular}{|l|c|c|c|}
\cline { 2 - 4 } \multicolumn{1}{c|}{} & \multicolumn{2}{c|}{$R=2$} & $R=3$ \\
\cline { 2 - 4 } \multicolumn{1}{c|}{} & $M=2$ & $M=4$ & $M=2$ \\
\hline Best coherent scheme & 100 & 85 & 50 \\
\hline Best non-coherent scheme & 115 & 165 & 60 \\
\hline
\end{tabular}

\subsection{Grassmannian signaling in multi-channel frequency-flat MIMO systems}

\subsubsection{System model}

Consider an OFDM communication system with $M$ transmit antennas and independent and identically distributed Gaussian noise at each of its $N$ receive antennas. The normalized channel coherence time, denoted by $T_{c}$, represents the duration in number of channel uses over which the channel coefficients are essentially constant. Similarly, the normalized channel coherence bandwidth, denoted by $B_{c}$, represents the bandwidth in number of subchannels over which the channel coefficients are essentially constant. In this Thesis, we refer to systems in which $B_{c}>1$ as broadband and the ones in which $B_{c}=1$ as narrowband. The duration of each channel use, $T_{s}$, spans one OFDM symbol and the subchannel bandwidth, $B_{s}$, corresponds to the bandwidth of one OFDM subchannel. The system utilizes Grassmannian signaling, described in Section 4.3.3, for high SNR communications over a block Rayleigh fading channel in which the channel coefficients assume independent realizations between consecutive blocks. For each block of duration $T_{c}$, a symbol, $\mathbf{X}^{(k)} \in \mathbb{C}^{T_{c} \times M}$, is transmitted over a subchannel $k \in\left\{1, \cdots, B_{c}\right\}$. The $(t, m)$-th entry of $\mathbf{X}^{(k)}$ 
represents the transmitted pulse at time $t \in\left\{1, \cdots, T_{c}\right\}$, by transmit antenna $m \in\{1, \cdots, M\}$, over subchannel $k$. In such subchannel, the channel gains matrix and the received symbol are denoted by $\mathbf{H}^{(k)} \in \mathbb{C}^{M \times N}$ and $\mathbf{Y}^{(k)} \in \mathbb{C}^{T_{c} \times N}$, respectively, where

$$
\mathbf{Y}^{(k)}=\mathbf{X}^{(k)} \mathbf{H}^{(k)}+\sqrt{\frac{M}{\rho T}} \mathbf{W}^{(k)} .
$$

The $(t, n)$-th entry of $\mathbf{Y}^{(k)}$ represents the received pulse at time $t$, by the $n \in\{1, \cdots, N\}$ receive antenna, and $\mathbf{W}^{(k)} \in \mathbb{C}^{T_{c} \times N}$ is the AWGN matrix with zero mean and unit variance. Although Grassmannian is capacity-achieving in systems with $B_{c}=1$, it incurs a significant loss in the achievable rate when independently utilized over individual subchannels with $B_{c}>1$.

\subsubsection{Degradation of independent narrowband Grass- mannian signaling}

Practical measurements show that, with standard subchannel bandwidths, the channel coefficients are stationary over multiple adjacent subchannels, thereby yielding $B_{c}>1$. To see that independent narrowband Grassmannian signaling incurs a loss in achievable rates, we define the number of communication degrees of freedom, $\psi$, of a $M \times N$ MIMO system that utilizes Grassmannian signaling for communications over a block-fading channel with $B_{c}=1$ and coherence time $T_{c}$ as $[33]$

$$
\psi_{T_{c}}=M\left(1-\frac{M}{T_{c}}\right) .
$$

In contrast, when the channel coefficients are stationary over a $T_{c} \times B_{c}$ timefrequency block, the number of degrees of freedom can be seen to be

$$
\psi_{T_{c} B_{c}}=M\left(1-\frac{M}{T_{c} B_{c}}\right) .
$$

Hence, utilizing narrowband Grassmannian signaling results in the loss of

$$
\psi_{T_{c} B_{c}}-\psi_{T_{c}}=\frac{M^{2}}{T_{c}}\left(1-\frac{1}{B_{c}}\right)
$$

degrees of freedom. In the following sections, we will investigate two techniques that can be used to overcome this drawback. The first technique is based on reshaping the transmitted pulse, whereas the second technique is based on concatenating the subchannels within $B_{c}$. 


\section{CHAPTER 4. RADIO SOLUTION: NON-COHERENT COMMUNICATIONS SCHEMES}

\subsubsection{Reshaping of the transmitted pulse}

In broadband systems, the underlying transmit filter can be modified to yield an equivalent narrowband system with a longer $T_{c}$, thus enabling the implementation of Grassmannian signaling without reducing $\psi_{T_{c} B_{c}}$. To elaborate, from a spectral perspective, since the bandwidth occupied by a pulse of duration $T_{s}, B_{s} \approx \frac{1}{T_{s}}$, the transmit filter of the original system can be modified to generate pulses with reduced durations, thereby occupying the entire coherence bandwidth and rendering $B_{c}=1$. Now, from a temporal perspective, reducing the duration of the transmit pulses increases the number of channel uses spanned by the normalized coherence time of the channel; i.e., the ratio of the channel physical coherence time to the duration of a channel use.

From the above discussion, it can be seen that elementary adjustment of the pulse shape enables the number of (temporal) channel uses of $T_{c}$ to be increased. However, applying this approach in practice has two major drawbacks. First, in current OFDM cellular systems, $B_{s}$ is selected such that it ensures backward compatibility between LTE systems and their HSPA predecessors is guaranteed by setting $B_{s}=15 \mathrm{kHz}$. Second, increasing $B_{s}$ results in decreasing the OFDM symbol duration, which subsequently reduces its efficiency. This drawback is because the OFDM symbol efficiency is given by the ratio of the duration of the cyclic prefix to that of the OFDM symbol. The duration of the cyclic prefix depends solely on the channel delay spread but is otherwise independent of $T_{s}$. Hence, decreasing $T_{s}$ reduces the OFDM symbol efficiency. Given these relatively stringent constraints on $B_{s}$, a more practical approach to enable efficient utilization of narrowband Grassmanning signaling in systems with $B_{c}>1$ is to utilize a technique that is independent of $B_{s}$. Such a technique is developed in the following section.

\subsubsection{Concatenated Grassmannian signaling}

In this section, we propose a technique whereby the subchannels within $B_{c}$ are concatenated to obtain an equivalent narrowband channel. This concatenation does not affect the underlying subchannel bandwidths and thus, can be readily applied to current and future cellular systems. After concatenation, the normalized channel block duration of the equivalent narrowband system, $T_{c, e q v}=T_{c} B_{c}$. Similarly, the normalized coherence bandwidth of the equivalent system, $B_{c, e q v}=1$. An illustration of the subchannel concatenation is depicted in Figure 4.10 when $T_{c}=B_{c}=2$

The system model of the equivalent narrowband system can be obtained by modifying the expression in (4.8) as follows: 


\subsection{Grassmannian signaling in multi-channel frequency-flat MIMO}

systems

$$
\tilde{\mathbf{Y}}=\tilde{\mathbf{X}} \mathbf{H}+\sqrt{\frac{M}{\rho T}} \tilde{\mathbf{W}},
$$

where

$$
\begin{aligned}
\tilde{\mathbf{Y}} & =\left[\mathbf{Y}^{(1)^{T}} \cdots \mathbf{Y}^{\left(B_{c}\right)^{T}}\right]^{T}, \\
\tilde{\mathbf{X}} & =\left[\mathbf{X}^{(1)^{T}} \cdots \mathbf{X}^{\left(B_{c}\right)^{T}}\right]^{T}, \\
\tilde{\mathbf{W}} & =\left[\mathbf{W}^{(1)^{T}} \cdots \mathbf{W}^{\left(B_{c}\right)^{T}}\right]^{T},
\end{aligned}
$$

where $\tilde{\mathbf{Y}}$ is the $T_{c, e q v} \times N$ received signal matrix, $\tilde{\mathbf{X}}$ is the $T_{c, e q v} \times M$ transmitted Grassmannian symbol, and $\tilde{\mathbf{W}}$ is the $T_{c, e q v} \times N$ AWGN matrix. Since the blockfading channel is assumed to be constant over a $T_{c} \times B_{c}$ time-frequency block, the channel coefficients matrix, $\mathbf{H}$, is constant across all subchannels within $B_{c}$; i.e., $\mathbf{H}^{(k)}=\mathbf{H}, \forall k \in\left\{1, \cdots, B_{c}\right\}$.

Having transformed the broadband channel into an equivalent narrowband one, capacity achieving communication at high SNRs can be established using Grassmannian signaling, but the dimensions of each Grassmannian symbol are $T_{c, e q v} \times M$, instead of $T_{c} \times M$. However, unlike the narrowband case in which each Grassmannian symbol is sent independently over one subchannel, in the equivalent system, each Grassmannian symbol is transmitted over all the subchannels within $B_{c}$. In particular, each $T_{c, \text { eqv }} \times M$ Grassmannian symbol is partitioned into $T_{c} \times M$ submatrices each of which is then transmitted on one of the subchannels within $B_{c}$. This introduces a structure between the signal matrices transmitted over different subchannels, thus enabling the utilization of all the available $\psi_{T_{c} B_{c}}$ degrees of freedom. At the receiver side, the $T_{c, e q v} \times M$ signal matrix can be recovered by detecting a concatenation of the submatrices received on the $B_{c}$ subchannels.

To elaborate on the capacity-achieving property of the proposed technique, we note that it was shown in [33] that Grassmannian signaling achieves the high SNR non-coherent block-fading channel capacity of narrowband systems; i.e., it reaches the capacity of the equivalent system after concatenation. However, the concatenation that underlies the proposed technique is a reversible operation

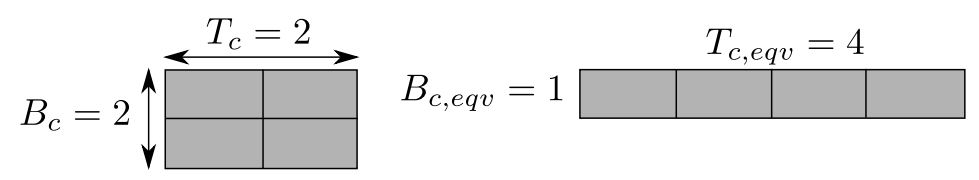

Figure 4.10: Concatenation of subchannels for a system with $B_{c}=2$ and $T_{c}=2$. IEEE (C) 2014 . 


\section{CHAPTER 4. RADIO SOLUTION: NON-COHERENT COMMUNICATIONS SCHEMES}

that does not affect capacity. In other words, the proposed method enables a broadband system to communicate at the high SNR block-fading channel capacity. The technique also allows the application of Grassmannian signaling in MIMO fast fading channels as elucidated in the following section.

\subsubsection{Grassmannian signaling in fast fading channels}

In this section, we consider the application of Grassmannian in fast fading channels; i.e., systems with $T_{c}=1$ channel use. In such system, the channel assumes an independent realization in each channel use. One example is the Tone-Hopping OFDM system in which each user hops pseudorandomly across all subchannels. Since space-time Grassmannian signalling, requires that $M \leq\left\lfloor\frac{T_{c}}{2}\right\rfloor[33]$; i.e., $M=0$. Therefore, in this case, this scheme is inapplicable in Tone-Hopping OFDM systems. However, if the channel coefficients are constant across multiple adjacent subchannels the concatenation technique of Section 4.4 .4 can be used for space-frequency Grassmannian signaling. The equivalent single-channel system in that case has $T_{c, \text { eqv }}=B_{c}$ and its high $\mathrm{SNR}$ ergodic non-coherent capacity is maximized with $M=\left\lfloor B_{c} / 2\right\rfloor$.

\subsubsection{Comparison between concatenated and indepen- dent narrowband Grassmannian signaling}

When $M \leq N$ and $T_{c} \geq \min (M, N)+N$, the Grassmannian signaling scheme can achieve the ergodic high SNR non-coherent narrowband channel capacity given by [33], whose spectral efficiency is as follows:

$$
\begin{aligned}
C(S N R) & =M\left(1-\frac{M}{T_{c}}\right) \log _{2} S N R \\
& +\frac{1}{T_{c}} \log _{2}\left(\pi^{M\left(T_{c}-M\right)} \prod_{i=1}^{M} \frac{(i-1) !}{(i+T-M-1)}\right) \\
& +\left(1-\frac{M}{T_{c}}\right) \sum_{i=N-M+1}^{N} \mathbb{E}\left[\log \chi_{2 i}^{2}\right]+o(1) .
\end{aligned}
$$

where $\chi_{2 i}^{2}$ is a chi-square random variable with $2 i$ degrees of freedom.

In this section, we provide a numerical comparison between the spectral efficiency of concatenated and independent narrowband Grassmannian signaling schemes when utilized in systems with $B_{c}>1$. We also evaluate the approximate spectral efficiency of concatenated Grassmannian signaling in an LTE UMi scenario [46]. In particular, we consider a UE moving at a speed of $25 \mathrm{~km} / \mathrm{h}$ in an LTE UMi scenario and communicating with the BS at a carrier 
frequency of $1.8 \mathrm{GHz}$. For this UE, the Doppler frequency $f_{d}=41.67 \mathrm{~Hz}$ and the $T_{c, e q v}$ can be evaluated using the frequency and time correlation functions as $[76]$

$$
\begin{gathered}
R(\Delta f)=\sum_{i=1}^{L} P_{i} e^{j 2 \pi \Delta f \tau_{i}}, \\
R(\Delta t)=J_{0}\left(2 \pi f_{d} \Delta t\right),
\end{gathered}
$$

where $L$ is the number of taps or paths of the multipath channel and, $P_{i}$ and $\tau_{i}$ are the power and the delay excess of the path $i$ th, respectively. Here, $\Delta t$ and $\Delta f$ are the signaling duration and bandwidth over which the channel correlation is evaluated. Given the LTE subchannel bandwidth, the spectral correlation $R(\Delta f)=0.999 \approx 1$ for a block of six subchannels; i.e., a $90 \mathrm{kHz}$ bandwidth. Similarly, the OFDM duration in LTE is $71.4 \mu \mathrm{s}$, which gives rise to a temporal correlation of $R(\Delta t)=0.99 \approx 1$ for a block of $T_{c}=8$ channel uses; i.e., $T_{c, \text { eqv }}=T_{c} B_{c}=48$.

We consider three channel scenarios all with $M=N=4$ antennas and $T_{c}=8$ channel uses but with $B_{c}=3,6$, and 10 subchannels. In Figure 4.11, we show the maximum achievable spectral efficiency by both, the narrowband and the concatenated Grassmannian signaling schemes in a broadband system. We also depict the LTE peak spectral efficiency at 64 -QAM and 5/6 code rate with only the channel estimation overhead taken into consideration [77].

From Figure 4.11, it can be seen that the concatenated time-frequency Grassmannian signaling is always superior to the independent utilization of subchannels within $B_{c}$ in the narrowband Grassmannian scheme of [33]. For instance, at an SNR of $30 \mathrm{~dB}$, a gain of $75 \%$ in the spectral efficiency can be observed for the case in which $B_{c}=3$ and $T_{c}=8$. This gain is due to the increase in $\psi$ degrees of freedom and approaches $M^{2} / T_{c}$ for asymptotically large $B_{c}$. Furthermore, concatenated Grassmannian signaling is superior to the LTE peak spectral efficiency. This result is because, in LTE, about $15 \%$ of the resources are allocated for training symbols to acquire the CSI when $M=4$. We note that the pulse shaping technique can also obtain the performance gains of the concatenated Grassmannian signaling but at the expense of changing the underlying transmit filter of the OFDM system and subsequently its symbol efficiency. 


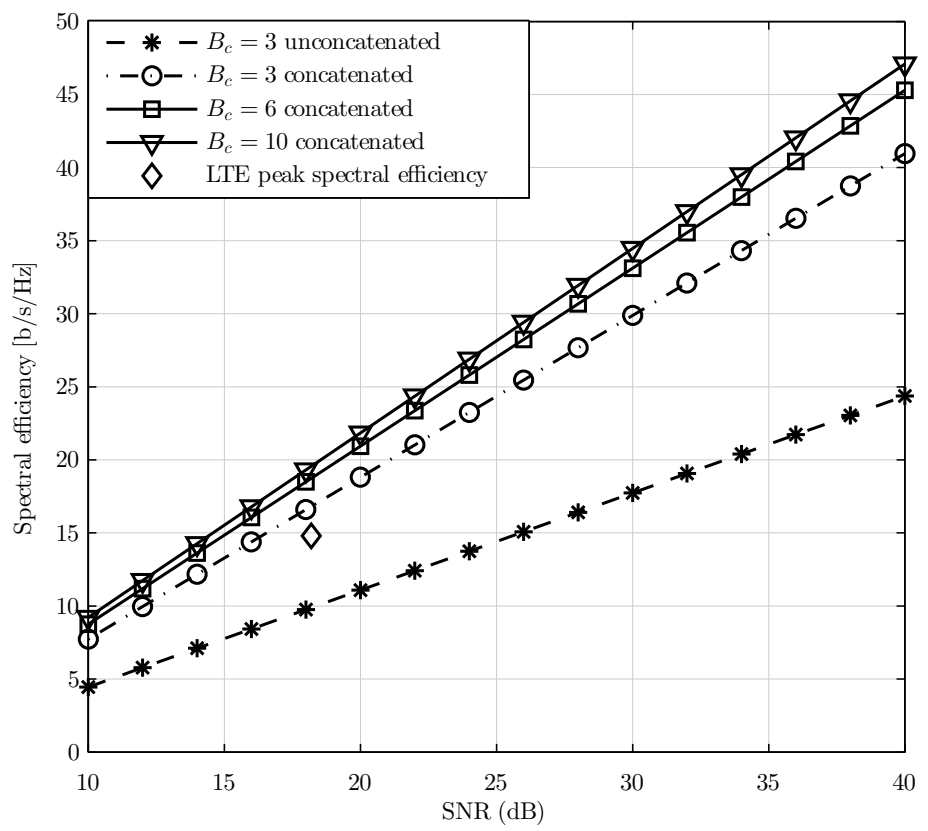

Figure 4.11: Spectral efficiency of concatenated and narrowband Grassmannian with $M=N=\frac{T_{c}}{2}$; LTE peak spectral efficiency also depicted. IEEE (C) 2014.

\subsection{Comparison of coherent and non-coherent schemes: multi-channel MIMO systems}

\subsubsection{Frequency-time mapping of Grassmannian codes}

Grassmannian signaling enables, at high SNR, rates approaching the noncoherent ergodic capacity provided a block-fading Rayleigh MIMO channel $[33,34]$. In this channel, the coefficients are constant during $F$ Resource Elements $(\mathrm{REs})^{3}$ and then change to a new realization. In practical channel conditions, this feature is not fulfilled, especially in a vehicular environment where the channel may vary quickly. However, in the next section we show that if Grassmannian signaling is transmitted in resources where the channel remains reasonably constant, significant performances are still obtained.

\footnotetext{
${ }^{3}$ In the 3GPP terminology, one RE consists of one subcarrier during one OFDM symbol.
} 


\subsection{Comparison of coherent and non-coherent schemes: multi-channel MIMO systems}

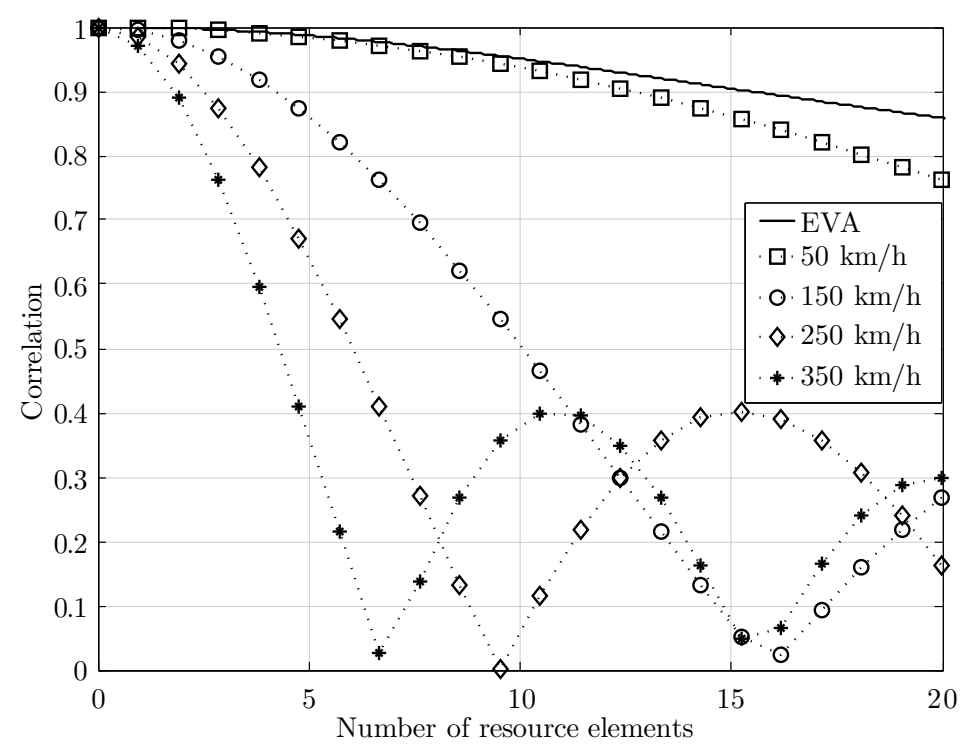

Figure 4.12: Comparison between temporal and frequency correlation.

Therefore, the best approach is to map Grassmannian blocks into the available time-frequency resources where the channel is as invariant as possible. The channel variability in LTE systems can be quantified with the frequency and time correlation functions shown in Equations (4.17) and (4.18).

Next, we evaluate the frequency correlation function in the Extended Vehicular A (EVA) scenario defined by the 3GPP [78] and the time correlation function at 50, 150, 250, and $350 \mathrm{~km} / \mathrm{h}$ assuming $f_{c}=2.6 \mathrm{GHz}$. Note that the Grassmannian blocks can be mapped in three different ways. First, given an OFDM symbol, the block is mapped along $F$ subcarriers. Second, given a subcarrier, the block is mapped along $F$ OFDM symbols. Third, the block is mapped in $S$ subcarriers during $T$ OFDM symbols, where $S \cdot T=F$.

Figure 4.12 shows the frequency-domain and time-domain correlation functions with the above considerations. Both functions decrease as the separation between resources, measured in REs, increases. However, the decreasing is more pronounced in the time domain. In particular, assuming a 90\% correlation criterion for a quasi-static channel, the channel in the frequency domain fulfills this target along 15 REs. For the time-domain channel and speed values of 50 , 150,250 , and $350 \mathrm{~km} / \mathrm{h}$, the number of REs with quasi-static channel conditions is $12,4,2$, and 1 , respectively. It is worth noting that the LTE technology 


\section{CHAPTER 4. RADIO SOLUTION: NON-COHERENT COMMUNICATIONS SCHEMES}

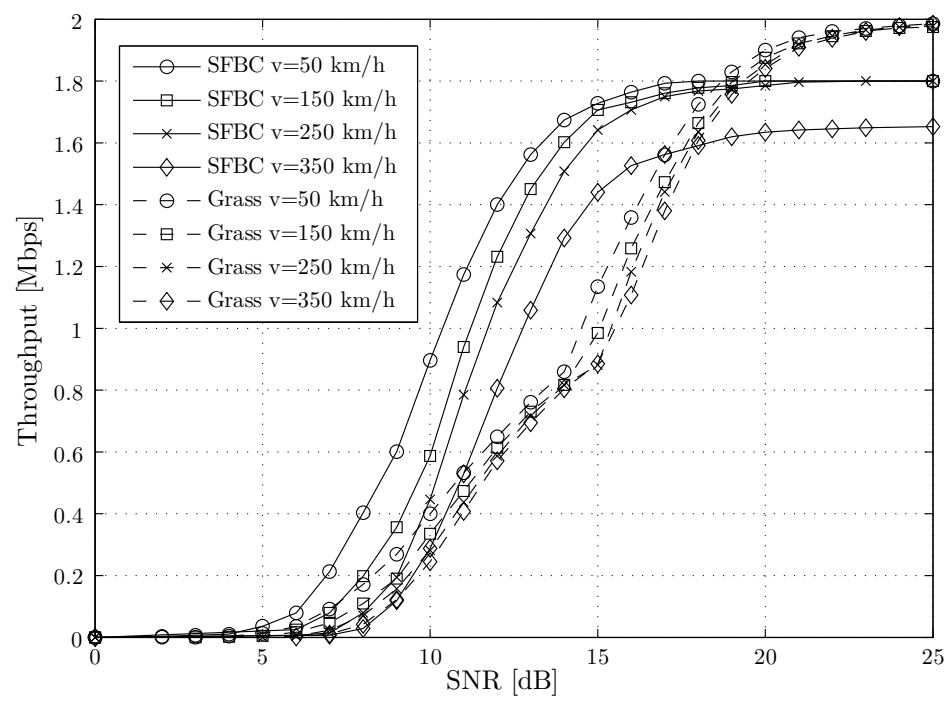

Figure 4.13: Performance comparison among coherent and non-coherent schemes for two uncorrelated antennas $(2 \times 2$ MIMO system $)$.

must support high speeds up to $350 \mathrm{~km} / \mathrm{h}$. Therefore, from these results, we can conclude that the frequency resource mapping is the most suitable option for Grassmannian signaling under vehicular channel conditions since it offers the highest number of REs where the channel can be considered quasi-static. Moreover, this resource allocation scheme is backward compatible with LTE. Therefore, for the rest of the chapter, we assume the Grassmannian blocks to be located in consecutive subcarriers within the same OFDM symbol.

\subsubsection{Performance analysis over time and frequency vary- ing spatially correlated channels}

The primary objective of this section is to compare the performance of coherent and non-coherent communication schemes under realistic conditions of channel variability and antenna correlation. This section firstly describes the simulation setup and then discusses these performance results.

\section{Simulation environment}

To evaluate the behavior of the different schemes, simulations have been conducted in an LTE link-level simulator calibrated in the European projects 


\subsection{Comparison of coherent and non-coherent schemes: multi-channel MIMO systems}

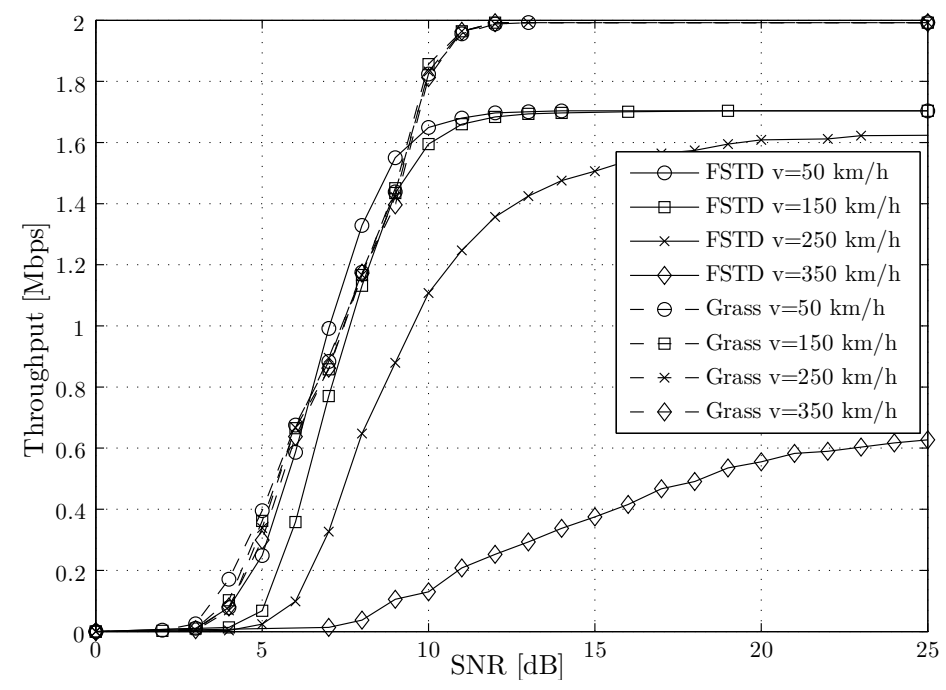

Figure 4.14: Performance comparison among coherent and non-coherent schemes for four uncorrelated antennas $(4 \times 4$ MIMO system).

WINNER + [59] and METIS [38]. The simulation was run for six resource blocks $(1.4 \mathrm{MHz})$ over the EVA $3 \mathrm{GPP}$ channel at a frequency of $2.6 \mathrm{GHz}$ [78]. We neither assume channel coding nor retransmissions of erroneous subframes. The LTE baselines are the transmit diversity modes SFBC for two antennas and FSTD for four antennas, with Quadrature Phase-Shift Keying (QPSK) modulation. Both coherent baselines consider a channel estimator based on a Wiener filtering in frequency and in time domain [79]. This channel estimator knows the frequency and time correlation of the channel, as well as the noise power, and uses the standardized LTE reference signals for channel estimation. At the receiver side, the coherent schemes decode the received signal with an MMSE detector. Conversely, the transmission based on Grassmannian signaling does not rely on pilots and sends only data blocks of $F$ resource elements length. We used $F=6$ for two transmit antennas and $F=8$ for four transmit antennas, with one and two bits per RE.

We modeled transmit and receive antenna correlation using the matrices defined in [78], for low, medium, and high correlation (see Section A.2.4). Note that, according to $3 \mathrm{GPP}$, medium antenna correlation implies a higher correlation at the UE than in the base station. Besides, low correlation means having totally uncorrelated antennas. 


\section{CHAPTER 4. RADIO SOLUTION: NON-COHERENT COMMUNICATIONS SCHEMES}

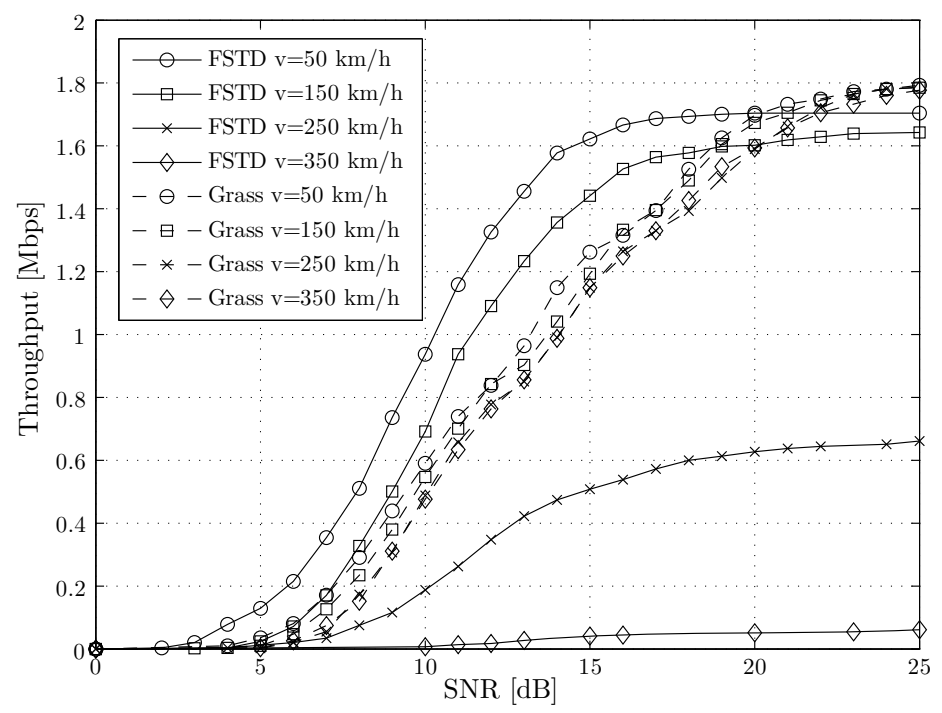

Figure 4.15: Performance comparison among coherent and non-coherent schemes with medium antenna correlation in a $4 \times 4$ setup.

\section{Results}

In Figure 4.13 and 4.14, we compare the coherent and non-coherent schemes in $2 \times 2$ and $4 \times 4$ setups with uncorrelated antennas. It is worth recalling that Grassmannian signaling operates optimally with uncorrelated antennas and constant channel along the Grassmannian block. However, in these results, the channel is varying in each sample according to the previously cited models. Focusing in the $2 \times 2$ setup, Figure 4.13 shows that, even though Grassmannian signaling is quite robust to mobility, the SFBC scheme outperforms the Grassmannian signaling except for high SNR. In fact, the non-coherent scheme transmits approximately $10 \%$ more data than the SFBC scheme since it saves the transmission of pilots. Because of this, in Figure 4.13, a $10 \%$ rate increase is observable at high SNR. On the other hand, Figure 4.14 shows the results for the $4 \times 4$ setup. The $4 \times 4$ Grassmannian approach is even more robust to mobility than the $2 \mathrm{x} 2$ case. Moreover, the FSTD scheme is highly degraded by the user speed. This degradation begins to be very significant for speed values starting from $150 \mathrm{~km} / \mathrm{h}$. The main reason for the FSTD degradation is that two out of the four antennas of the LTE system are using poorer channel estimates, as these antennas are only assigned $50 \%$ of the pilot symbols as compared with 


\subsection{Comparison of coherent and non-coherent schemes: multi-channel MIMO systems}

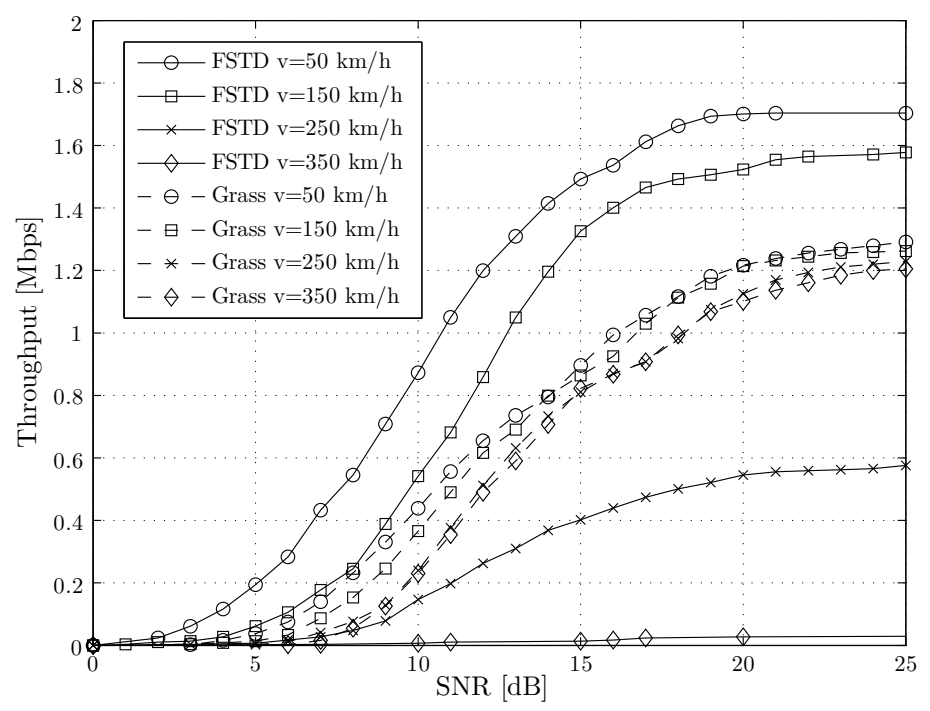

Figure 4.16: Performance comparison among coherent and non-coherent schemes with high antenna correlation in a $4 \times 4$ setup.

Table 4.3: Minimum user speed from which Grassmannian signaling outperforms FSTD.

\begin{tabular}{c|c}
\hline Correlation & $\mathbf{v}_{\min }$ \\
\hline \hline Low & 100 \\
\hline Medium & $150-200$ \\
\hline High & $200-250$ \\
\hline
\end{tabular}

the other two antennas. Note also that, at high SNR, Grassmannian signaling obtains $15 \%$ more data rate than the FSTD scheme. These results suggest that Grassmannian signaling is a promising technique for high-speed scenarios with a high-enough number of transmit antennas since, in these cases, the removal of pilots entails an important increase of available resources while suffering less degradation from users' mobility.

To totally eliminate the conditions beneficial for the Grassmannian signaling scheme, and to complete the performance analysis under realistic conditions, we incorporated the effect of antenna spatial correlation within the $4 \times 4$ setup. Figures 4.15 and 4.16 show the obtained throughput for medium and high an- 


\section{CHAPTER 4. RADIO SOLUTION: NON-COHERENT COMMUNICATIONS SCHEMES}

tenna correlation, respectively. It can be observed that antenna correlation affects severely to both coherent and non-coherent schemes. Nevertheless, at high speeds, low temporal correlation strongly degrades the channel estimation used in the FSTD and, therefore, Grassmannian signaling has superior performance. To complement this result, we set an objective throughput of $1 \mathrm{Mbps}$ to analyze the minimum speed from which Grassmannian signaling outperforms FSTD for different levels of correlation. Table 4.3 shows that this minimum value increases as the antenna correlation augments since Grassmannian signaling relies on antenna uncorrelation. For medium and high correlations, we found that the minimum speeds correspond to those in motorways or high-speed trains. Overall, with the resource mapping proposed in this Thesis, Grassmannian signaling was shown to be especially affected by antenna correlation and not that much by user mobility, while coherent schemes are more sensitive to temporal correlation, mostly when increasing the number of antennas.

\subsection{Multi-user MIMO transmission and recep- tion with Grassmannian signaling}

\subsubsection{System model}

We consider a MIMO downlink transmission from a BS with $M$ antennas to $U$ UE devices with $N$ antennas each. We assume a transmission through blockfading channels $\mathbf{H}_{u} \in \mathbb{C}^{M \times N}, u=1, \ldots, U$, containing Gaussian i.i.d. elements with zero mean and unit variance, which are unknown at both the transmitter and receiver sides. The channel block length is assumed to be $T$ consecutive channel uses. The signal transmitted to user $u$ through the $M$ antennas during one channel block is represented by the matrix $\mathbf{X}_{u} \in \mathbb{C}^{T \times M}$.

The possible transmitted matrices are uniformly drawn from a finite-length constellation $\mathcal{C}=\left\{\mathbf{S}_{i}, i=1, \ldots,|\mathcal{C}|\right\} \subset \mathbb{C}^{T \times M}$, the elements of which have covariance $\boldsymbol{\Gamma}_{\mathrm{X}}$ and, for simplicity, zero mean. For further ease of exposition, in this Thesis, we assume all users' signals are drawn from the same constellation. Nevertheless, the results presented in this Thesis could be easily extended to other constellations with non-zero mean elements and, also, to different peruser constellations.

One of the main objectives of this Thesis is to provide MU communication with non-coherent reception. Following the rationale of [80], in the Gaussian broadcast channel with multiple receivers, superposing messages with different power levels improves the achievable rates in comparison with time sharing procedures, provided that successive decoding at the receivers is feasible [81]. With the aim of validating this conclusion under non-coherent detection, in this work 


\subsection{Multi-user MIMO transmission and reception with Grassmannian signaling}

we combine superposition coding [80] with Grassmannian Constellations (GC), i.e. the transmitted signal is a superposition of independent GC signals. To this end, the BS power has to be divided among the users. We define different per-user power sharing factors $\gamma_{u}, u=1, \ldots, U$, so that $\sum_{u=1}^{U} \gamma_{u}=1$. We further consider an SNR equal to $\rho_{u}$ at user $u$, where the power contributions of all the users are included within the desired signal power term. User $u$ is assumed to know $\rho_{u}, \gamma_{\ell}$ for $\ell=1, \ldots, U$, and also the constellation. The received signal matrix for user $u, \mathbf{Y}_{u} \in \mathbb{C}^{T \times N}$, is

$$
\mathbf{Y}_{u}=\left(\sum_{\ell=1}^{U} \sqrt{\frac{\gamma_{\ell} \rho_{u} T}{\operatorname{Tr}\left(\boldsymbol{\Gamma}_{\mathrm{X}}\right)}} \mathbf{X}_{\ell}\right) \mathbf{H}_{u}+\mathbf{Z}_{u}
$$

where $\mathbf{Z}_{u} \in \mathbb{C}^{T \times N}$ is the AWGN matrix with i.i.d. zero-mean unit-variance elements.

\subsubsection{Non-coherent multi-user receiver}

In this section, we address the non-coherent ML detection rule for the investigated MU scheme and provide a quantitative analysis of its computational cost. Then, a suboptimal detection scheme suitable for the considered superposition coding setup is proposed. It is shown to reduce the ML detector computational cost.

\section{Maximum-likelihood non-coherent multi-user detection}

Focusing on the data detection at a certain user $u$, the symbol detected by the ML receiver is

$$
\hat{\imath}_{u}=\arg \max _{i} f_{\mathbf{Y}_{u}}\left(\mathbf{\Upsilon} \mid \mathbf{X}_{u}=\mathbf{S}_{i}\right),
$$

where $f_{\mathbf{Y}_{u}}\left(\mathbf{\Upsilon} \mid \mathbf{X}_{u}=\mathbf{S}_{i}\right)$ is the conditional probability distribution of $\mathbf{Y}_{u}$ knowing that $\mathbf{S}_{i}$ has been sent to the user $u$ from the constellation $\mathcal{C}$, and $\boldsymbol{\Upsilon}$ denotes a particular value taken by the random variable $\mathbf{Y}_{u}$. In the MU framework, the detection of the signal of user $u$ depends on the values of the symbols for the other users. In this sense, for the sake of simplicity, we introduce $\mathbf{X}=\left(\mathbf{X}_{1}, \ldots, \mathbf{X}_{U}\right) \in \mathcal{C}^{U}$, which contains all the users' signals. We further introduce $\mathbf{X}_{-u}=\left(\mathbf{X}_{1}, \ldots, \mathbf{X}_{u-1}, \mathbf{X}_{u+1}, \ldots, \mathbf{X}_{U}\right) \in \mathcal{C}^{U-1}$ that contains all the users' signals except the signal of the user $u$. Since $\mathcal{C}$ is a finite-length constellation with equiprobable symbols, so are $\mathcal{C}^{U}$ and $\mathcal{C}^{U-1}$.

Taking into account these considerations, $f_{\mathbf{Y}_{u}}\left(\mathbf{\Upsilon} \mid \mathbf{X}_{u}=\mathbf{S}_{i}\right)$ can be obtained as follows:

$$
f_{\mathbf{Y}_{u}}\left(\mathbf{\Upsilon} \mid \mathbf{X}_{u}=\mathbf{S}_{i}\right)=\frac{1}{\left|\mathcal{C}^{U-1}\right|} \sum_{\mathbf{A} \in \mathcal{C}^{U-1}} f_{\mathbf{Y}_{u}}\left(\mathbf{\Upsilon} \mid \mathbf{X}_{-u}=\mathbf{A}, \mathbf{X}_{u}=\mathbf{S}_{i}\right) .
$$




\section{CHAPTER 4. RADIO SOLUTION: NON-COHERENT} COMMUNICATIONS SCHEMES

For the Gaussian random channel, the conditioned probability terms in (4.21) follow a Gaussian distribution [31], that is,

$$
f_{\mathbf{Y}_{u}}(\boldsymbol{\Upsilon} \mid \mathbf{X}=\mathbf{B})=\frac{\exp \left(-\operatorname{Tr}\left(\boldsymbol{\Upsilon}^{\mathrm{H}}\left(\boldsymbol{\Sigma}\left(\mathbf{B}, \rho_{u}\right)\right)^{-1} \boldsymbol{\Upsilon}\right)\right)}{\pi^{T N} \operatorname{det}^{N}\left(\boldsymbol{\Sigma}\left(\mathbf{B}, \rho_{u}\right)\right)}
$$

where, $\boldsymbol{\Sigma}\left(\mathbf{B}, \rho_{u}\right)=\mathbb{E}\left[\mathbf{Y}_{u} \mathbf{Y}_{u}^{\mathrm{H}} \mid \mathbf{X}=\mathbf{B}\right]$ denotes the conditioned covariance matrix of the received signal $\mathbf{Y}_{u}$. The term $\boldsymbol{\Sigma}\left(\mathbf{B}, \rho_{u}\right)$, which is calculated in Section D.1 has the following expression:

$$
\boldsymbol{\Sigma}(\mathbf{B}, \rho)=\frac{N \rho T}{\operatorname{Tr}\left(\boldsymbol{\Gamma}_{\mathrm{X}}\right)} \sum_{m=1}^{U} \sum_{n=1}^{U} \sqrt{\gamma_{m} \gamma_{n}} \mathbf{B}_{m} \mathbf{B}_{n}^{\mathrm{H}}+N \mathbf{I}_{T} .
$$

Here, the notation $\mathbf{B}_{j}$ has been used to reference the $j$-th $T \times M$-dimensional block of $\mathbf{B}$, and $\mathbf{I}_{T}$ denotes the $T \times T$ identity matrix.

Let us now focus on the computational burden of the derived non-coherent MU ML detector. It can be observed that the ML detection of each user's signal in this MU framework involves the computation of (4.21) a number of times equal to $|\mathcal{C}|$, i.e. one per constellation symbol. Hence, this detection technique leads to an overall per user cost of $|\mathcal{C}|^{U}$ evaluations of the function (4.22) and, implicitly, also of (4.23). Note that such a high computational cost, which increases exponentially with the number of users, is impractical for real-world communication systems. Besides, the constellation size has also a big impact on the detection complexity, which may prevent practical systems from transmitting at high rates due to this burdensome detection stage.

\subsubsection{Proposed non-coherent multi-user detection}

To reduce the ML detection complexity, we propose a suboptimal MU receiver adapted to the superposition coding transmission scheme. The key feature of this receiver is that each user detects its signal after the previous detection of the signals of other users, which are received with higher power than its own. To perform this successive detection, an approximated system model is considered at each detection step. It is important to note that, due to the fact that the channel coefficients are unknown, conventional successive interference cancelation is not possible at each detection stage to remove the effect of previously detected user signals. Rather, previously detected symbols are considered within the detection rule. The approximated system model and proposed detection scheme are next described.

Without loss of generality, $\gamma_{1}>\ldots>\gamma_{U}$ is assumed, which means that user $u$ receives the signals for users $1, \ldots, u-1$ with higher power than its signal and 


\subsection{Multi-user MIMO transmission and reception with}

Grassmannian signaling

the signals for users $u+1, \ldots, U$ with lower power. In the proposed detection scheme, user $u$ detects consecutively, and prior to its signal, the signals of users $1, \ldots, u-1$, which are received with higher power than its own. For the detection of user $p$, the signals for users $p+1, \ldots, U$ are considered as noise and, hence, are grouped within the AWGN term in the system model. Hence, user $u$ will consider the following approximated expression of the received signal for the detection of the signal for user $p$ :

$$
\widetilde{\mathbf{Y}}_{u, p}=\left(\sum_{\ell=1}^{p} \sqrt{\frac{\gamma_{\ell} \rho_{u}^{\prime} T}{\operatorname{Tr}\left(\boldsymbol{\Gamma}_{\mathbf{X}}\right)}} \mathbf{X}_{\ell}\right) \mathbf{H}_{u}+\mathbf{Z}_{u},
$$

where the power levels of users $p+1, \ldots, U$ reduce the original SNR to $\rho_{u}^{\prime}=$ $\rho_{u} /\left(1+\sum_{\ell=p+1}^{U} \gamma_{\ell} \rho_{u}\right)$.

Considering the approximated system model (4.24) and introducing the function

$$
G(\boldsymbol{\Upsilon}, \mathbf{B}, \rho)=\frac{\exp \left(-\operatorname{Tr}\left(\boldsymbol{\Upsilon}^{\mathrm{H}}(\boldsymbol{\Sigma}(\mathbf{B}, \rho))^{-1} \boldsymbol{\Upsilon}\right)\right)}{\pi^{T N} \operatorname{det}^{N}(\boldsymbol{\Sigma}(\mathbf{B}, \rho))},
$$

the proposed detection rule to calculate the symbol for user $p$ by user $u$ is

$$
\hat{\imath}_{u, p}=\arg \max _{i} G\left(\mathbf{\Upsilon},\left(\mathbf{S}_{\hat{\imath}_{u, 1}}, \ldots, \mathbf{S}_{\hat{\imath}_{u, p-1}}, \mathbf{S}_{i}, \mathbf{0}, \ldots, \mathbf{0}\right), \rho_{u}^{\prime}\right),
$$

where $\mathbf{0}$ stands for a matrix containing $T \times M$ zero elements. Recall that $\boldsymbol{\Sigma}(\mathbf{B}, \rho)$ denotes the conditioned covariance matrix described in (4.23).

Overall, (4.26) is an approximation of (4.20) that, instead of summing all the probability terms (4.22) for every possible constellation values for every user, it takes into account only the particular values of $\hat{\imath}_{u, 1}, \ldots, \hat{\imath}_{u, p-1}$, which are already known due to previous detection steps at user $u$. Note that, at every user, the detection of the signal of user 1 simply solves a Single-User (SU) detection problem, since the rest of users are considered within the noise term. The covariance matrix in (4.23) is reduced to

$$
\boldsymbol{\Sigma}\left(\left(\mathbf{S}_{i}, \mathbf{0}, \ldots, \mathbf{0}\right), \rho_{u}^{\prime}\right)=\frac{N \rho_{u}^{\prime} \gamma_{u} T}{\operatorname{Tr}\left(\boldsymbol{\Gamma}_{\mathrm{X}}\right)} \mathbf{S}_{i} \mathbf{S}_{i}^{\mathrm{H}}+N \mathbf{I}_{T} .
$$

After substituting (4.27) into (4.25) and assuming unitary codebooks, the maximization problem in (4.26) turns into the following equivalent detection rule for user 1 :

$$
\hat{\imath}_{u, 1}=\arg \max _{i} \operatorname{Tr}\left(\mathbf{\Upsilon}^{\mathrm{H}} \mathbf{S}_{i} \mathbf{S}_{i}^{\mathrm{H}} \mathbf{\Upsilon}\right),
$$

which corresponds to the SU non-coherent ML receiver considered in [82] and [34]. Section D.2 calculates the SU non-coherent ML receiver of Equation 4.28. Since unitary codebooks are assumed in this work, the ML receiver is equivalent to the well-known GLRT receiver [82]. 


\section{CHAPTER 4. RADIO SOLUTION: NON-COHERENT COMMUNICATIONS SCHEMES}

Remark. If the users transmit different constellations, in the $M U M L$ detector, each user must know the constellations of the $U$ users. In the proposed suboptimum detector, however, user $u$ needs only to know the constellations of users $p=1, \ldots, u-1$ for their previous detection.

Regarding computational aspects, we next evaluate the detection complexity of the worst-case user, i.e. user $U$, which must detect $U-1$ signals prior to its own. In this case, the proposed detector performs $U|\mathcal{C}|$ evaluations of the function $G(\boldsymbol{\Upsilon}, \mathbf{B}, \rho)$, that is, $|\mathcal{C}|$ function evaluations for each detected user. Therefore, even for the worst-case user, the per-user cost is reduced from exponential to linear with the number of users. Furthermore, note that for users $1, \ldots, U-1$, the calculation of the covariance matrix is far less computationally demanding than in the initial non-coherent MU ML detector, due to all the components of $\mathbf{B}$ that are set to zero. As a result, the proposed MU detector is a meaningful scheme to provide non-coherent detection with much fewer complexity than the exhaustive-search-based ML detection scheme.

\subsubsection{Downlink non-coherent multi-user gain}

In this section, we highlight a special feature of downlink non-coherent MU communications based on superposition coding. We will study this feature using a simplified two-user system model. Let

$$
\mathbf{Y}=\mathbf{W}+\mathbf{X}_{2} \mathbf{H}
$$

be the received signal, where

$$
\mathbf{W}=\mathbf{X}_{1} \mathbf{H}+\mathbf{Z},
$$

$\mathbf{X}_{1}$ and $\mathbf{X}_{2}$ are the independent signals transmitted to the two users, $\mathbf{H}$ is the channel matrix, and $\mathbf{Z}$ is the AWGN. Let $\mathbf{X}_{1}$ be the desired signal to be detected from the received signal $\mathbf{Y}$. Note that $\mathbf{W}$ corresponds to the received signal in a case where only $\mathbf{X}_{1}$ is present in the system ( $\mathrm{SU}$ case), and $\mathbf{Y}$ can be seen as a post-processed version of $\mathbf{W}$, due to the addition of $\mathbf{X}_{2} \mathbf{H}$.

We first want to prove that, in a coherent system, the signals of the rest of users can never enhance the detection performance of the desired user signal. In other words, other signals in the system are only seen as an additional interference. As observed from (4.29) and (4.30), if $\mathbf{H}$ and $\mathbf{W}$ are known, knowing $\mathbf{X}_{1}$ does not provide any extra information about $\mathbf{Y}$, i.e. $\mathbf{X}_{1}$ and $\mathbf{Y}$ are conditionally independent and $f_{\mathbf{Y}}\left(\mathbf{Y} \mid \mathbf{W}, \mathbf{X}_{1}, \mathbf{H}\right)=f_{\mathbf{Y}}(\mathbf{Y} \mid \mathbf{W}, \mathbf{H})$. Therefore, the random variables $\mathbf{X}_{1}, \mathbf{W}$, and $\mathbf{Y}$ form the Markov chain $\mathbf{X}_{1} \rightarrow \mathbf{W} \rightarrow \mathbf{Y}$. As in any other Markov chain, the data processing theorem must be fulfilled, which states that processing $\mathbf{W}$ can not add new information about $\mathbf{X}_{1}$, i.e., 
the data processing inequality must hold $I\left(\mathbf{X}_{1} ; \mathbf{W} \mid \mathbf{H}\right) \geq I\left(\mathbf{X}_{1} ; \mathbf{Y} \mid \mathbf{H}\right)$. As a consequence, superposing $\mathbf{X}_{2} \mathbf{H}$ over $\mathbf{W}$ (4.29) results in no more capacity in comparison with the case where only $\mathbf{X}_{1}$ is transmitted (4.30). Hence, the capacity of user 1 in the MU coherent setup is never higher than in the SU coherent setup.

Now we focus on the downlink non-coherent case. If $\mathbf{W}$ is known but $\mathbf{H}$ is unknown, $\mathbf{Y}$ still has a dependence on $\mathbf{X}_{1}$ through $\mathbf{H}$. In this non-coherent case, the knowledge about $\mathbf{X}_{1}$ and $\mathbf{W}$ can provide some extra information about the channel matrix $\mathbf{H}$, or, equivalently, $f_{\mathbf{Y}}\left(\mathbf{Y} \mid \mathbf{W}, \mathbf{X}_{1}\right) \neq f_{\mathbf{Y}}(\mathbf{Y} \mid \mathbf{W})$. Therefore, the variables $\mathbf{X}_{1}, \mathbf{W}$, and $\mathbf{Y}$ do no longer form a Markov chain, as opposed to what happens in the coherent scenario. Consequently, the data processing inequality does not necessarily hold and both conditions $I\left(\mathbf{X}_{1} ; \mathbf{W}\right) \geq I\left(\mathbf{X}_{1} ; \mathbf{Y}\right)$ and $I\left(\mathbf{X}_{1} ; \mathbf{W}\right)<I\left(\mathbf{X}_{1} ; \mathbf{Y}\right)$ are attainable. This means that, in the downlink MU non-coherent setup, it is also possible to increase the information about $\mathbf{X}_{1}$ by processing $\mathbf{W}$. Note that a more detailed study of the situations where $I\left(\mathbf{X}_{1} ; \mathbf{W}\right)<I\left(\mathbf{X}_{1} ; \mathbf{Y}\right)$ is out of the scope of this Thesis. Nevertheless, in the following section, we will show through simulations an exemplary case where the BLER of an individual user in a two-user setup is reduced with respect to the SU case. This happens when there is a high difference between the power of the two users; the signal $\mathbf{X}_{2}$ increases the channel knowledge for the detection of $\mathbf{X}_{1}$.

\subsubsection{Results}

In this section some numerical results that show the performance of the proposed MU detection schemes is presented. A block-fading Gaussian channel constant during $T=4$ time slots is considered to evaluate the transmission from $M=2$ antennas to $U=2$ users with $N=2$ antennas each. The selected codebook for both users is a GC of 16 points, obtained using the direct design proposed in [34]. Note that the use of this codebook achieves a maximum transmission rate of $R_{\mathrm{c}}=1 \mathrm{bpcu}$ ( 4 bits are transmitted in 4 time slots).

The proposed suboptimum non-coherent MU detector was compared with its ML counterpart, and both schemes were also compared with a non-coherent user TDM scheme. To do this, we obtained the Effective Transmission Rates (ETRs) of both users, defined from the BLER as ETR $=R_{\mathrm{c}}(1-\mathrm{BLER})$. The ETR regions of the three evaluated schemes are depicted in Figure 4.17 for a fixed SNR value for both users equal to $\rho_{u}=10 \mathrm{~dB}, u=1,2$. For the first two schemes we set $0 \leq \gamma_{1} \leq 1$ and $\gamma_{2}=1-\gamma_{1}$ to obtain the desired range of BLER values. It can be observed that the two setups carrying out non-coherent MU detection strongly outperform the TDM scheme in terms of effective sumrate, reinforcing the interest of MU non-coherent transmission. Note that the maximum transmission rate of $1 \mathrm{bpcu}$ is never reached in Figure 4.17 since the 


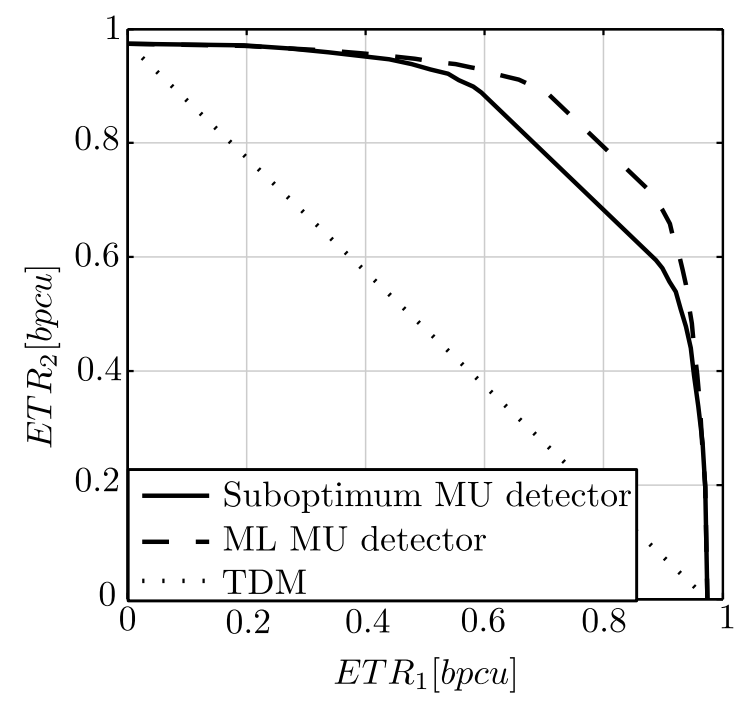

Figure 4.17: Effective transmission rates in a two-user non-coherent downlink communication with GC using TDM, superposition coding with ML MU detection and superposition coding with proposed MU detection. IEEE (C) 2014.

minimum BLER of SU detection is never zero at $\rho_{u}=10 \mathrm{~dB}$. Other $\rho_{u}$ values will lead to different maximum effective user rates for each independent user.

Next, we studied the additional MU gain attained in the downlink noncoherent setup. To this end, the BLER performance of user 1 was evaluated for a set of detectors, fixing the transmitted power of user 1 to $0 \mathrm{dBW}$ and varying the transmitted power of user 2 within a range of values. In Figure 4.18, the BLER values with both the proposed MU non-coherent receiver and the ML MU detector were compared with the SU case in which only non-coherent transmission to user 1 happens. It can be observed that non-coherent SU transmission is the best performance achieving scheme for user 2 transmitted power values lower than $5 \mathrm{dBW}$. For transmitted power values of user 2 higher than this value, the ML MU non-coherent detector has better BLER performance, meaning that user 1 can benefit from the transmission to user 2 to enhance its data detection. The proposed MU detector also exhibits some performance gain over the SU case at transmitted power values of user 2 higher than $18 \mathrm{dBW}$. It can be also observed that the proposed reduced-complexity MU detector approaches the performance of ML detection, especially as the difference between the power-sharing factors of both users increases. We highlight here that the 
latter results support the main conclusion that is drawn from Section 4.6.4 regarding the additional MU gain attained in downlink non-coherent systems. Recall that this is only part of the overall gain shown in Figure 4.17, which already showed the advantage of MU non-coherent communication against TDM.

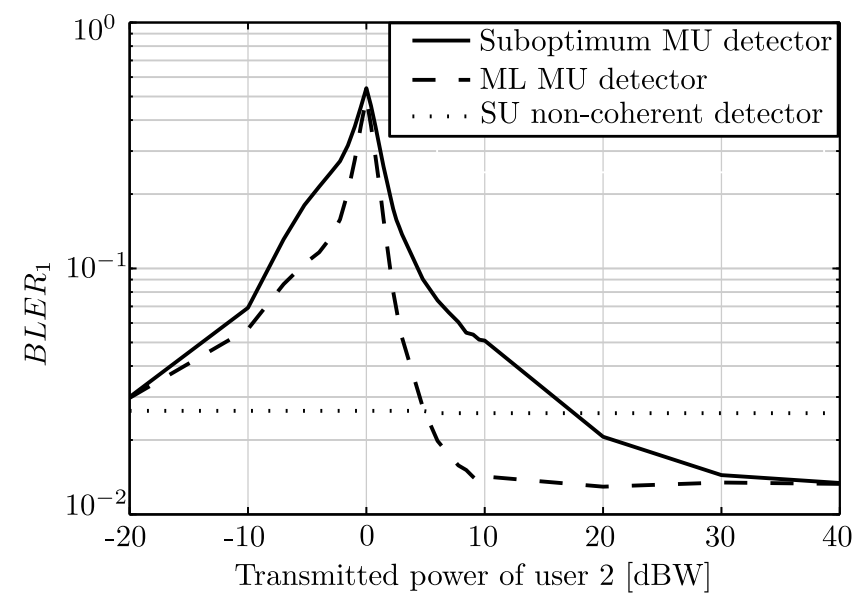

Figure 4.18: BLER performance for the detection schemes under study, as a function of the transmitted power of user 2. IEEE (C) 2014.

\subsection{Integration of Grassmannian signaling for the future $5 \mathrm{G}$ communication}

One scenario for the application of Grassmannian signaling is the classical cellular link where users mobility is high. Imagine that multiple vehicular users are moving at a certain speed in an urban street or motorway. The users receive a multiple-stream (MIMO) data connection from a cellular base station without being assigned orthogonal resources. The use of Grassmannian signaling makes the communication with the vehicular users more robust to channel variations, and it also saves those resources that would be allocated to pilot transmission in a coherent system.

However, this classic scenario is not the only of interest. As discussed in Chapter 2, direct communication between devices requires them to be synchronized and to exchange specific reference to perform channel estimation at the receiver. First approaches in the standard highlight the need for at least two OFDM symbols per subframe to transmit reference signals with only one an- 


\section{CHAPTER 4. RADIO SOLUTION: NON-COHERENT COMMUNICATIONS SCHEMES}

tenna port. This represents $15 \%$ of the available resources and could be even worse in the case of usage of multiple antenna elements. Moreover, the reasonable proximity of D2D links also points towards a high SINR situation, in which Grassmannian signaling has shown all its potential. It is here, where distributed and uncontrolled selection of pilots may cause a substantial problem of pilot contamination, where non-coherent communications present their maximum interest.

Of particular relevance is the case of $\mathrm{V} 2 \mathrm{~V}$ communication, where channel state is highly dynamic, and channel estimation error may make communication infeasible with conventional coherent communications schemes. Here the integration of Grassmannian signaling shows enormous potential most especially when the number of transmitters increases. Imagine that multiple vehicles are moving at a certain speed in an urban street or motorway. Assuming a conventional coherent communication between multiple vehicles, the signals from multiple vehicles overlap, and their pilot signals for channel estimation interfere with each other. In fact, this problem worsens as the number of transmitting vehicles increases, which could be the case of being in a traffic jam or a road with multiple lanes at a rush hour. In these scenarios, the non-coherent MultiUser MIMO (MU-MIMO) transmission scheme proposed in Section 4.6 avoids the pilot pollution problem and, at the same time, allows for the spatial multiplexing of several data flows transmitted to different users or vehicles, which increase link capacity.

Finally, Grassmannian signaling is well suited for the multi-point coordinated transmission, provided that different columns of the equivalent codeword matrix can be transmitted from a distributed antenna system. With proper synchronization, the transmission effort could be divided into a group of transmission points, hence increasing the equivalent power available for the link. In the multi-hop scenario this represents the situation in which the base station first transmits the data to the mobile relay/s, including joint transmission details, and then, in a second hop, all involved transmission points broadcast the signal to the final destination. Given that no pilot signal is needed, the assistant transmission points act like phantom transmitters from the end user perspective, which is only aware of the existence of the main base station. This phantom cell concept [83], widely discussed in the 3GPP forum, fits perfectly with the blind transmission mode that Grassmannian signaling enables.

\subsubsection{CA and the integration of non-coherent carriers}

IMT-Advanced technologies incorporate, as one of their key features, the aggregation of the continuous or discontinuous spectrum to achieve wider bandwidth and consequently increase transmission capability. This concept is known as 
Carrier Aggregation (CA). The main characteristics of the first version of CA included as part of 3GPP LTE-Advanced Release 10 are described in [84]. In 2012, the 3GPP technical specification group dedicated to the Radio Access Network (RAN) started discussing some CA enhancements to be included in future releases of the standard. Some of the ideas out of this discussion are not included yet in the standards but are part of the solution that this Thesis proposes for the integration of the non-coherent signaling into $5 \mathrm{G}$ systems.

\section{CA in LTE-Advanced Release 10}

LTE-Advanced supports the aggregation of up to five $20 \mathrm{MHz}$ Component Carriers (CoCs). CA is designed to support aggregation of a variety of different arrangements of CoCs, including CoCs of the same or different bandwidths, adjacent or non-adjacent CoCs in the same frequency band, and CoCs in different frequency bands.

All CoCs in LTE-Advanced Release 10 are designed to be backward-compatible. This means that each CoC is fully accessible to legacy LTE UEs. Therefore, essential Release 8 channels and signals such as synchronization signals and system information are transmitted on each CoC. Nevertheless, some mechanisms such as cell barring can be used to avoid legacy LTE UEs to camp on a particular CoC.

Therefore, each CoC appears as a separate cell with its Cell ID. A UE that is configured for CA is connected to one Primary serving Cell (PCell) and up to four Secondary serving Cells (SCells). Firstly, the UE establishes the radio access connection with a serving cell that becomes the PCell. This PCell plays an essential role in security, mobility information, and connection maintenance. SCells may be configured after connection establishment, just to provide additional radio resources. When adding a new SCell, dedicated signaling is used to send all the required system information for the new SCell.

With this set of CoCs, SCells can be activated or deactivated to reduce UE power consumption in LTE-Advanced CA. If a UE is configured with one or more SCells, the eNB can activate and deactivate the configured SCells sending a MAC command. In addition, a UE maintains a timer per configured SCell and automatically deactivates the associated SCell upon its expiry. When a SCell is deactivated, the UE stops monitoring that $\mathrm{CoC}$. Activation/deactivation is not applicable for the PCell that must remain active during the whole LTE-Active state.

The activation/deactivation timing is carefully defined to ensure a perfect synchronization between the eNB and the UE. When a UE receives a MAC control element activating a SCell, the SCell has to be ready for operation after 


\section{CHAPTER 4. RADIO SOLUTION: NON-COHERENT COMMUNICATIONS SCHEMES}

8 frames $(8 \mathrm{~ms})$. At this moment, the SCell deactivation timer is started. Note that this timer can be set to infinity to avoid automatic deactivation.

Moreover, it is worth noting that, at the physical layer, each transport block is mapped into a single CoC. Even if a UE is simultaneously scheduled on multiple CoCs, HARQ, modulation, coding, and resource allocation are performed independently on each $\mathrm{CoC}$. Each downlink $\mathrm{CoC}$ carries the same control signaling region at the start of each subframe as in Release 8/9. The $\mathrm{PDCCH}$ allocates downlink resource to users at the same $\mathrm{CoC}$, but it can also perform cross-carrier scheduling, which allows scheduling data transmissions on another CoC.

Therefore, according to Release 10, it is feasible to configure a particular $\mathrm{CoC}$ to operate with a different transmission scheme, which opens the door for the integration of non-coherent communication systems, but still CoCs in this release must be backward-compatible with LTE Release 8 users. This fact prevents us from removing the CRS from the resource grid, which is the main benefit of using non-coherent communications.

\section{CA enhancements beyond Release 10}

After the introduction of CA in Release 10, a 3GPP Technical Specification Group on RAN started a work item to improve the operation of CA. This work item focused on several topics, namely, support for multiple uplink timing advance values, intra-band non-contiguous $\mathrm{CA}$, support of inter-band $\mathrm{CA}$ for Time Division Duplexing (TDD), and the definition of additional carrier types. Among these topics, one of the key components is the New Carrier Type (NCT), focused on non-backward compatible carrier types, which were already considered during Release 10 standardization, but they were left for later releases. The NCT is currently being completed in Release 13.

The main motivation for the definition of the NCT is the use of carriers with minimized transmission of legacy control signaling and CRS, which would reduce interferences and signaling overhead. NCT supports both non-standalone and standalone use cases. On the one hand, a non-standalone carrier must be aggregated to a legacy LTE carrier, where carrier segments and extension carriers are the main candidates. On the other hand, a standalone carrier could be used without a connection with a legacy LTE carrier. Besides, as the NCTs are non-backward compatible carriers, they are not accessible by legacy LTE UEs.

Carrier segments are defined as bandwidth extensions of a legacy LTE carrier and constitute a mechanism to expand bandwidth, process also known as band filling. With carrier segments, additional resource blocks are aggregated to the legacy carrier while still keeping the backward compatibility of the orig- 
inal carrier. The advantage of carrier segments is that no additional PDCCH resources are required. Moreover, these segments can be smaller than legacy LTE bandwidths. Carrier segments are always adjacent and linked to a legacy carrier, thus, this legacy carrier is who provides synchronization and system information. They support the same HARQ process, PDCCH indication, and transmission mode as the legacy carrier.

An extension carrier must also be associated with a legacy LTE carrier because it has no control channels. Currently, two types of extension carriers are under discussion in 3GPP depending on the synchronization with the legacy carrier. An extension carrier may be considered synchronized with the legacy carrier only if they are co-located and the frequency separation is sufficiently small. In that case, synchronization signals and CRS do not need to be present on the synchronized extension carrier and the measurements for the extension carrier can also rely on the legacy carrier.

\section{Proposal for the implementation of non-coherent extension carriers}

The existence of Transmission Mode 9 and 10 using DeModulation Reference Signal (DM-RS) would still allow for channel estimation without CRS, that is, for data demodulation and CSI-RSs for radio channel quality feedback. Moreover, the new enhanced version of the PDCCH channel (E-PDCCH) in Release 11, would also play a crucial role in the integration since E-PDCCH, together with cross-carrier scheduling, allows removing the common downlink control channels from the NCT. Therefore, it would be feasible, using MAC signaling and the E-PDCCH, to activate a $\mathrm{CoC}$ dedicated to the transmission with Transmission Modes 9 and 10, or with non-coherent communications. In this last case, DM-RS would not be transmitted, with the subsequent saving in resource consumption. The MAC signaling should be extended to indicate to the user the use of a new Transmission Mode (TM-11), which shall be associated with the Grassmannian signaling. This represents a minor modification to the standard, as the continuous addition of new transmission modes in different releases has demonstrated.

\subsubsection{Effect of the imbalance among the powers received from distributed transmission points}

We consider a MIMO downlink transmission from a set of coordinated TPs with multiple antennas to a set of users. UEs are rarely located at the same distance from all the TPs involved in the transmission. Therefore, the signal is usually received with different power levels. Focusing on the transmission of GC matrices jointly from multiple TPs, we consider that there is a power 


\section{CHAPTER 4. RADIO SOLUTION: NON-COHERENT COMMUNICATIONS SCHEMES}

imbalance among the TP of $0 \leq \gamma \leq 1$ that weighs the columns (basis) of the transmitted constellation symbol in a different way. For an exemplary GC of $4 \times 2$ symbol matrices, which could be transmitted from two TPs with a single antenna each, the original constellation point is composed of two column vectors $\mathbf{x}_{m} \in \mathbb{C}^{4}$, for $m=1,2$

$$
\mathbf{X}_{i}=\left[\begin{array}{ll}
\mathbf{x}_{1} & \mathbf{x}_{2}
\end{array}\right]
$$

and the unbalanced constellation point is

$$
\mathbf{X}_{i}=\left[\begin{array}{ll}
\mathbf{x}_{1} \sqrt{1+\gamma} & \mathbf{x}_{2} \sqrt{1-\gamma}
\end{array}\right]
$$

where it can be noted that for $\gamma \neq 0$ the subspace basis is not longer orthonormal, it is just orthogonal. Figure 4.19 illustrates the transmission of two column vectors from two transmission points. For the performance evaluation, the following power difference among the TP (in $\mathrm{dB}$ ) will be assumed

$$
\Delta_{p}(d B)=10 \log \left(\frac{1+\gamma}{1-\gamma}\right)
$$

In this scenario, there is perfect cooperation among the TPs by sending different information to the same user, in such a way that the signal matrix is split among the set of cooperative transmitters. We evaluated the impact of the power imbalance over the received signal when the user is not at the same distance from all the TPs participating in the cooperative transmission. Figure 4.20 shows the BLER of the GC, Alamouti and Golden schemes for an SNR of $20 \mathrm{~dB}$, within a range of values of the power difference among TP. It can be seen that the power imbalance degrades the three schemes, but the GC still outperforms the Alamouti and Golden schemes. It is worth noting that
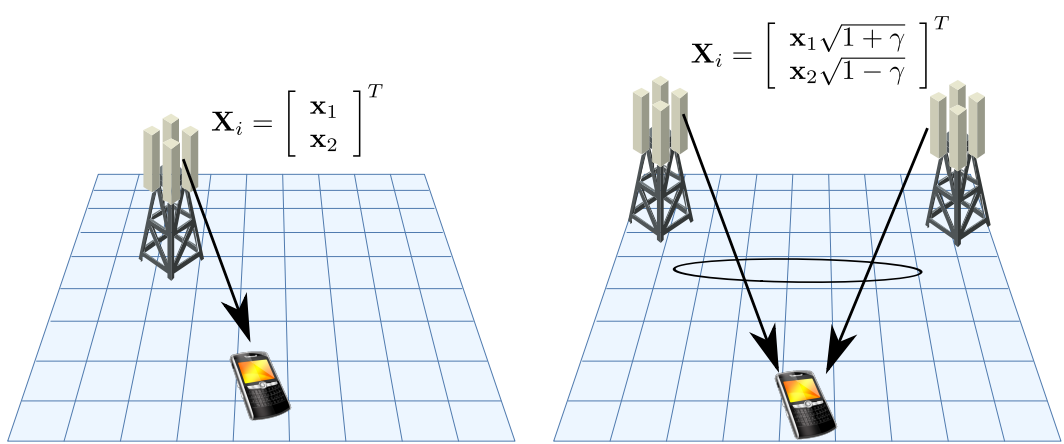

Figure 4.19: Transmission of GC matrices from multiple TPs. 
more than $17 \mathrm{~dB}$ of power imbalance are needed for the GC BLER performance be degraded one order of magnitude. Therefore, the evaluated schemes were shown to be robust to the power imbalance within a wide range of power imbalance values.

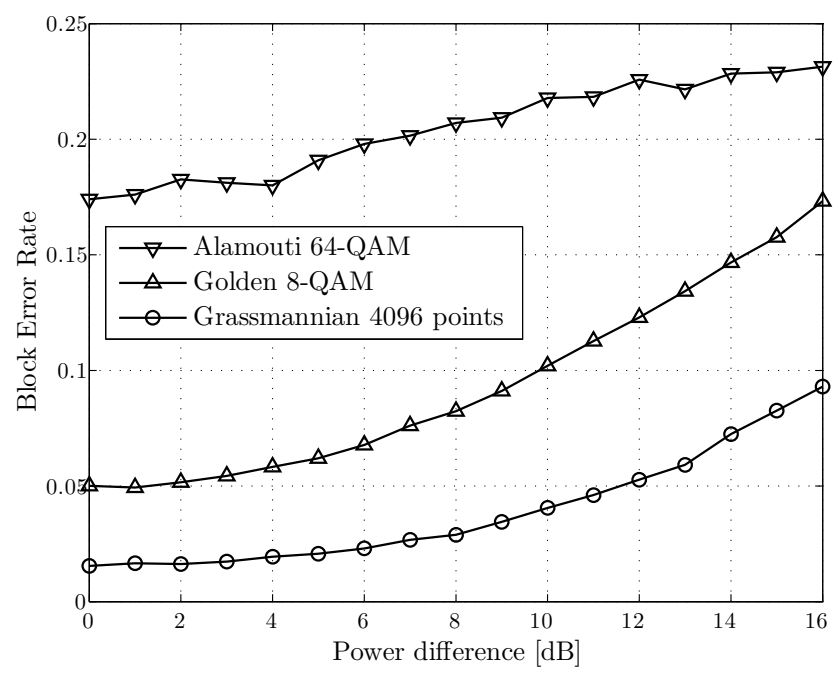

Figure 4.20: BLER performance of GC, Alamouti and Golden codes at $20 \mathrm{~dB}$ of SNR with increasing power difference between two TP.

\subsection{Conclusion}

In this chapter, a performance comparison between coherent and SU noncoherent signaling schemes under practical channel conditions has been carried out. The analysis carried out in this section for the SU scenario is focused on three major blocks structured from lower to higher complexity of the channel:

- Single-channel time-varying systems. For two transmit antennas, the performance of Grassmannian codes and DUSTM have been compared with two coherent benchmark schemes based on Alamouti and Golden codes. For four transmit antennas, orthogonal and quasi-orthogonal STBC have been used for comparison. At high SNR, our simulations show that, for a transmission rate of 2 bpcu and 2 transmit antennas, DUSTM offers the best performance for all the evaluated normalized 
Doppler frequency values. On the other hand, for a higher transmission rate, Grassmannian offers better performance than Alamouti, Golden code, and DUSTM schemes at low to medium values of $f_{d} T_{s}$. For four antennas, the performance advantage of non-coherent techniques is even higher, and the Grassmannian constellation of $T=8$ outperforms all others. We show that this signaling is a promising technique in scenarios with mobility and high SNR, especially for high transmission data rates and a high number of transmit antennas. This motivates its relevance in the design of new open-loop transmission techniques valid for higher-order MIMO configurations.

- Multi-channel block-fading systems. A concatenation technique that yields a spectrally-efficient time-frequency Grassmannian signaling scheme for MIMO frequency-flat broadband systems is proposed. The proposed technique introduces a structure between the signals transmitted over the subchannels within the coherence bandwidth, thus utilizing the correlation between the channel coefficients in both time and frequency domains. This eliminates the loss in the degrees of freedom incurred by using independent Grassmannian signaling over individual subchannels within the coherence bandwidth. Hence, this technique enables the system to communicate at the high SNR non-coherent MIMO block-fading channel capacity when the channel coefficients are constant over a time-frequency channel block. The proposed technique also renders Grassmannian signaling applicable in fast fading systems in which $B_{c}>1$. The proposed concatenation technique does not require changing the underlying pulse duration of the system and is, therefore, compatible with current and future systems.

- Multi-channel time and frequency varying spatially correlated systems. The integration of Grassmannian signaling into LTE systems including practical aspects such as user mobility and antenna correlation have been studied. Furthermore, an LTE-based resource mapping of Grassmannian signaling was proposed and evaluated for vehicular environments, over an LTE link-level simulator, fully compliant with LTE Release 8 and 9. For two antennas, we have compared the Grassmannian codes with SFBC and, for four transmit antennas, FSTD has been used for comparison. Overall, Grassmannian signaling is a promising technique for LTE transmit diversity for a high number of transmit antennas, even with antenna correlation. We showed that, although antenna correlation affects harder the Grassmannian signaling than the FSTD, the low temporal correlation strongly degrades the channel estimation needed for the 
FSTD scheme. After combining both effects, the Grassmannian signaling outperforms the FSTD for speeds higher than a minimum value.

Overall, non-coherent communications and, in particular, Grassmannian codes are promising techniques for high-rate open-loop communications with more than two transmit antennas time and frequency varying spatially correlated channels.

After this comparison, the use of superposition coding and a detection scheme is proposed to enable MU downlink communication in a non-coherent MIMO system. The ML detection rule was also derived, exhibiting exponential complexity with the number of users. In contrast, the complexity of the proposed suboptimum non-coherent MU detector is linear, involving a substantial complexity reduction. The presented MU non-coherent MIMO schemes were shown to increase the overall performance about TDM. Furthermore, downlink MU non-coherent detection can provide, in some cases, some gain in the individual user rates with respect to SU detection, thanks to channel knowledge obtained from transmissions to other users. Overall, this work showed that MU non-coherent detection is feasible and advantageous. The proposed approach is one step towards the implementation of MU non-coherent techniques in realworld cellular systems. This solution reinforces the interest of non-coherent schemes in the future $5 \mathrm{G}$. In this sense, Section 4.7 discusses the integration of Grassmannian signaling in the $5 \mathrm{G}$ architecture. 
CHAPTER 4. RADIO SOLUTION: NON-COHERENT COMMUNICATIONS SCHEMES 


\section{Chapter 5}

\section{Conclusions and future work}

\subsection{Concluding remarks}

This dissertation has investigated the integration of mobile relaying at network and radio level. Firstly, at the network level, this Thesis has proposed the MIH signaling for the management of multiple radio interfaces in a cellular system assisted with MRs. Our investigations have focused on the use of MIH signaling for the mobile relay selection with the objective of increasing coverage and data rates but minimizing signaling overhead. To this end, this dissertation has employed a simulation platform including link-level and system-level simulations. Secondly, at radio layer, this Thesis has proposed and analyzed the integration of non-coherent systems as a solution to the drawbacks of coherent systems such as pilot overhead, pilot contamination and performance degradation with mobility. The investigations have focused on single-user, multi-user, and multipoint non-coherent MIMO transmissions. With this aim, a link-level simulator has been employed to compare the performance of non-coherent schemes and training-based systems.

One of the central issues of mobile relaying is the relay selection and its integration in a cellular system. Results have shown an overall performance improvement when using the proposed MIH-driven relay selection mechanism. Besides, the proposed relay selection procedure is a low-cost solution because no modification of the cellular system architecture is required for its implementation. It is only necessary to carefully adjust the SINR threshold that triggers the selection of relay to cover user quality of service when the cellular system is 


\section{CHAPTER 5. CONCLUSIONS AND FUTURE WORK}

unable to do it. Moreover, we have proposed a simple technique that reduces the signaling overhead. Finally, we have shown the relevance of mobile relaying to reduce deployment costs in a cellular system. In a typical European city scenario, the reduction of CAPEX and OPEX is about $17.47 \%$ and $15.39 \%$, respectively.

Regarding the performance of non-coherent systems compared to coherent ones over single-channel temporally-correlated scenarios, we have identified that DUSTM offers, for two transmit antennas and low transmission rate, the best performance for all the evaluated normalized Doppler frequency values. For a higher transmission rate, Grassmannian signaling offers better performance than Alamouti, Golden codes, and DUSTM schemes at low to medium velocity. For four antennas, the performance gain of non-coherent techniques is even higher, and the Grassmannian constellation outperforms all others. This result motivates its relevance in the design of new open-loop transmission techniques valid for higher-order MIMO configurations.

In a multi-carrier MIMO system and high SNR, we have shown the importance of modeling the correlation between the channel coefficients both in time and frequency domains. To maximize capacity, we have proposed a concatenation technique that maps the transmitted matrix over the subchannels that are within the coherence bandwidth and coherence time. The proposed method also renders Grassmannian signaling applicable in fast fading systems with a coherence bandwidth higher than one subchannel. The proposed concatenation technique does not require changing the underlying pulse duration of the system and is, therefore, compatible with current and future wireless systems.

In a multi-carrier MIMO system with real channel conditions of mobility and antenna correlation, we point out that Grassmannian signaling is a promising technique for open loop transmission, mostly in vehicular scenarios and when the number of antennas is high enough. With four transmit antennas, the channel variability in time strongly degrades the channel estimation used in the LTE system, which makes Grassmannian signaling outperforms the coherent schemes starting from a certain speed, even with antenna correlation. However, results also show that this antenna correlation affects more severely the Grassmannian signaling, which further motivates the interest of focusing on vehicular communications where a massive number of antennas can be placed on the vehicle while ensuring high uncorrelation among them.

We have also proposed a transmission and reception mechanism for MU MIMO non-coherent communications. This proposal uses superposition coding and a suboptimum non-coherent MU detector, which exhibits linear complexity. This MU non-coherent MIMO scheme has shown to increase the overall performance about TDM. Furthermore, downlink MU non-coherent detection can provide, in some cases, some gain in the individual user rates with respect 
to $\mathrm{SU}$ detection. Overall, this work has shown that MU non-coherent detection is feasible and advantageous. The proposed approach is one step towards the implementation of MU non-coherent techniques in real-world cellular systems.

In a multi-point MIMO transmission case, we have also evaluated the impact of the power imbalance over the received signal when the user is not at the same distance from all the TP involved in the cooperative transmission. In particular, Grassmannian constellations outperformed the Alamouti and Golden codes, and its BLER degradation was less than one order of magnitude. Therefore, results pointed out that non-coherent transmission is robust within a broad range of imbalance power values.

Finally, with the use of a new carrier type we have identified how to integrate a new transmission mode based on non-coherent communications wherein neither the CRS nor the DM-RS are transmitted, with the consequent savings in resource consumption. This represents a minor modification to the standard, and only non-coherent codebooks should be included within this transmission mode, like SFBC for two antennas and FSTD for four antennas.

\section{$5.2 \quad$ Future research issues}

Several innovative research topics requiring further study have been identified in this Thesis. Some of these research directions are the following:

- Mobile relaying topic has not yet been fully covered by the $3 \mathrm{GPP}$ and needs to be extensively investigated for the future $5 \mathrm{G}$ as a natural evolution of fixed relaying. One of the very hot research topics related to mobile relaying is how to motivate users to cooperate since the consumption of battery still blocks the willingness of customers to accept this role. Although the evolution of mobile communication systems is to offer a global service anywhere and anytime with high data rates, it is worthless if these data rates can be only offered for some users at the expense of spending the battery of other users.

- Non-coherent schemes. In this dissertation, non-coherent schemes have been analyzed and compared with other space-time coding schemes implemented in the LTE standard. Before being ready for a standard adoption, non-coherent communications need to be further investigated in the following directions:

- New Grassmannian designs. Grassmannian signaling stands out for achieving, at high SNR, data rates approaching channel capacity. One of the design methods of the Grassmannian signaling is by 
numerical optimization designs, which present a high design complexity for medium and high size constellations. For these constellations, the required memory is exponential with the transmission rate and coherence time. This drawback is especially important at high SNR where, to achieve high data rates, one should construct high-rate constellations. Therefore, it is the paramount importance to analyze new design algorithms that offer similar or even higher performance than numerical optimization designs

- Decoding algorithms. In general, there does not exist simplified decoding algorithms that obtain ML performance. This fact causes that, all the matrices of the constellation have to be checked in the decoding algorithm. This entails enormous computational effort with limits the feasibility of this decoding method. Other decoding alternatives that approach optimum performance need to be further investigated.

- Antenna correlation. In the studies carried out in this dissertation, Grassmannian signaling has been especially affected by antenna correlation. This is mainly because this signaling was designed for uncorrelated antennas. New non-coherent designs should take into consideration the antenna correlation, especially, if mobile relaying is employed, since the dimensions of a UE device are often small.

- Comparison with spatial-multiplexing schemes. Throughout this dissertation, the performance comparisons of non-coherent systems with the coherent systems have been only performed with diversity training-based schemes. However, if new non-coherent schemes based on Grassmannian signaling could be implemented to achieve high data rates, this signaling could be compared with spatial-multiplexing MIMO schemes, which are specially designed for these rates.

- Channel coding. The research presented in this dissertation has not taken into account channel coding. To obtain a coding gain, the Grassmannian constellation should be appropriately labeled taking into account the geometry of the Grassmannian manifolds.

- Multi-user detection. Future work should also face the design of non-coherent communications schemes for the uplink, further complexity reductions in the MU detection stage and also finding the conditions where downlink MU non-coherent detection provides additional gain in the individual user rates with respect to SU detection. 


\section{Appendix A}

\section{Simulation tools}

As described in Section 1.5, given the enormous complexity of wireless systems, it is impossible to evaluate their performance using analytical methods. For this reason, computer simulation represents a real alternative for the assessment of these systems, achieving a good trade-off between complexity, cost, time of development, and accuracy. However, a complete simulation model would suppose a prohibitive computational burden, and this simulation is usually divided into two stages known as link level and system level. For this reason, the Mobile Communication Group (MCG) of the Institute of Telecommunications and Multimedia Applications (iTEAM) at the Universitat Politècnica de València (UPV) implemented a simulation tool composed of these two levels of abstraction.

\section{A.1 System-level simulator}

Figure A.1 illustrates the general structure of the system-level simulator. When the traffic generated at the application layer arrives at the Internet Protocol (IP) layer, the packets are distributed to the different radio interfaces depending on the selected link. On the one hand, in the Base Station (BS)-Relay Node (RN) connection, the packets are sent through the Long Term Evolution (LTE) technology. On the other hand, in the RN-Mobile Node (MN) connection, the packets are sent through the IEEE 802.11n technology.

\section{A.1.1 IEEE 802.11n}

The free space path loss equation for 802.11 Wireless Local Area Network (WLAN) line of sight links between the point $\mathrm{A}$ and the point $\mathrm{B}$ is defined 


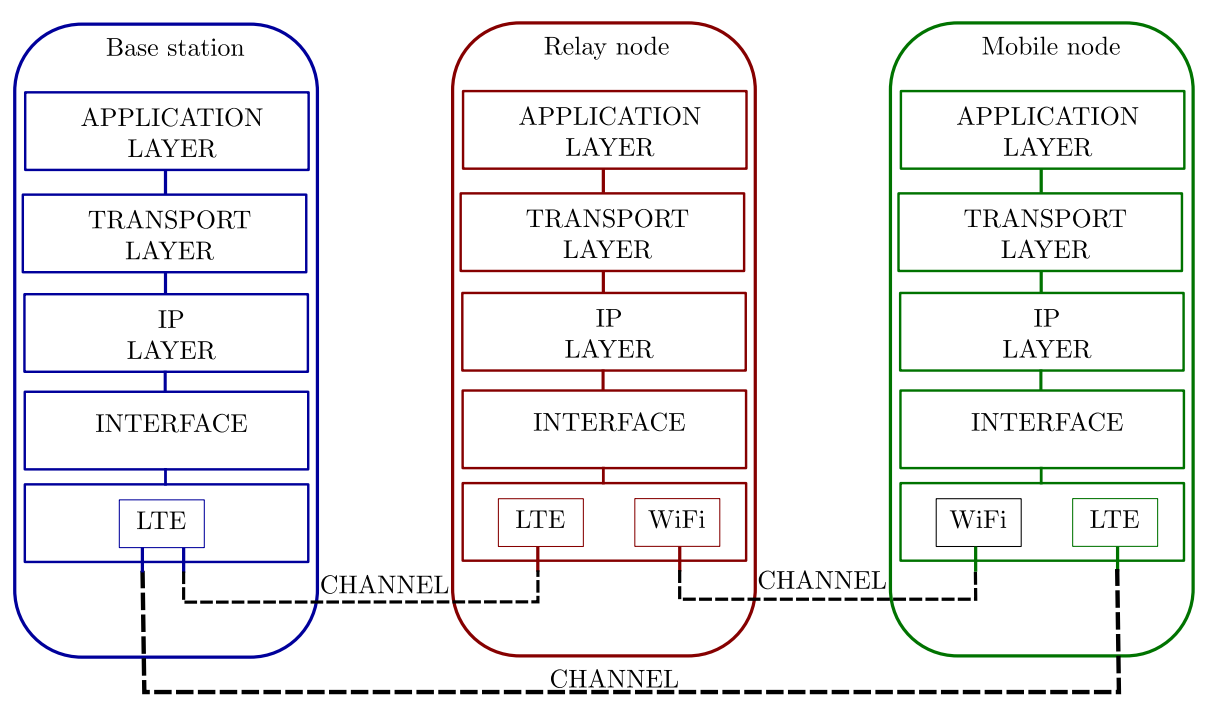

Figure A.1: System-level simulator general structure.

as $[61]$ :

$$
P_{\text {loss }}=40 \log _{10} d(m)+20 \log _{10} f(G H z)-20 \log _{10} h_{t}(m) h_{r}(m),
$$

where $d(m)$ is the distance in meters between the coordinate $\mathrm{A}$ and the coordinate $\mathrm{B}, f(G H z)$ is the carrier frequency in gigahertz, and $h_{t}(m)$ and $h_{r}(m)$ are the antenna height in meters of the transmitter and the receiver, respectively. The received power at the point $B$ is the transmitted power minus the path loss. Once the received power is calculated, the experimented throughput is obtained through Table A.1 for a bandwidth of $20 \mathrm{MHz}$ [56].

\section{A.1.2 LTE}

The received Signal to Interference plus Noise Ratio (SINR) Cumulative Distribution Functions (CDFs) after antenna receiver were obtained for the different evaluation scenarios: Suburban Macrocell (SMa), Rural Macrocell (RMa), Urban Macrocell (UMa), Urban Microcell (UMi), and Indoor Hotspot (InH). Figure A.2 illustrates the distribution of the SINR for those scenarios with cell radius of $20,67,167$, and 577 meters, respectively. For each user position there is an one-to-one correspondence with the SINR value according to Equation (3.1) and Figure A.2. Once the SINR is calculated, the equivalent throughput can be obtained through Figure A.3. 


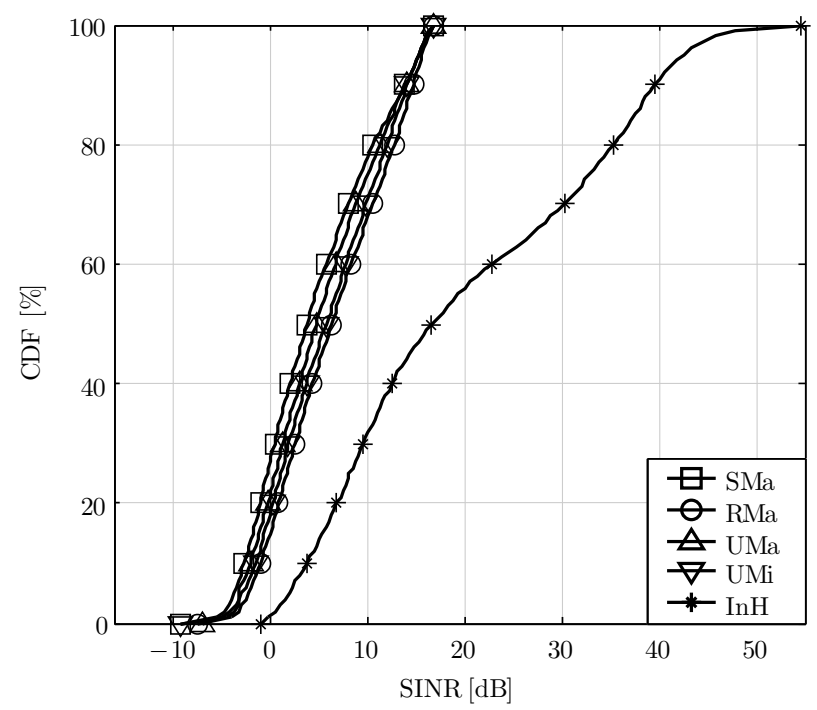

Figure A.2: Received SINR CDF after antenna receiver for the ITU scenarios.

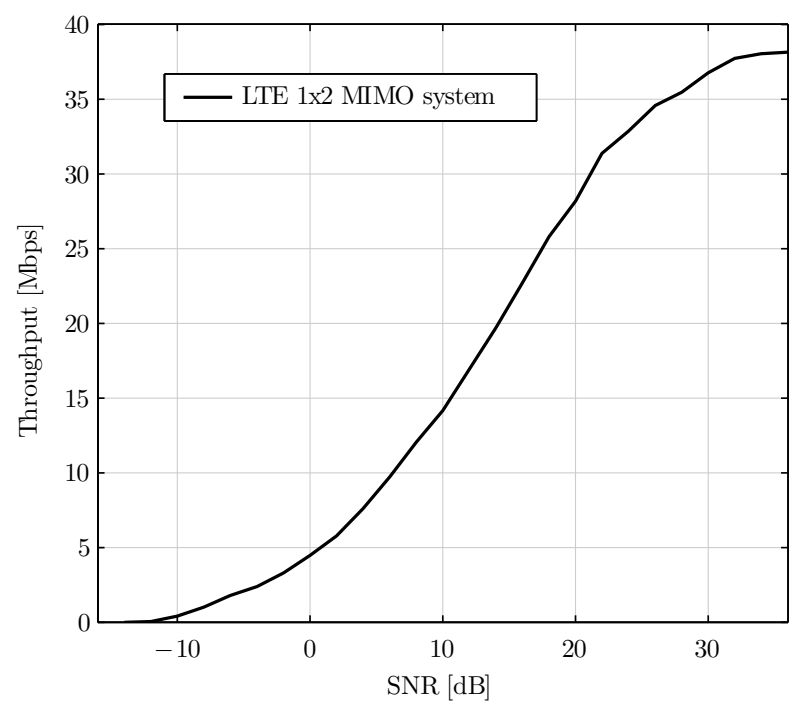

Figure A.3: Throughput versus SNR for an LTE 1x2 MIMO system. 
Table A.1: Experimented data rates versus receiver minimum input level sensitivity for a bandwidth of $20 \mathrm{MHz}$.

\begin{tabular}{|c|c|}
\hline Minimum sensitivity $\mathbf{( d B m )}$ & Data rates (Mbps) \\
\hline-82 & 6.5 \\
\hline-79 & 13 \\
\hline-77 & 19.5 \\
\hline-74 & 26 \\
\hline-70 & 39 \\
\hline-66 & 52 \\
\hline-65 & 58.5 \\
\hline-64 & 65 \\
\hline
\end{tabular}

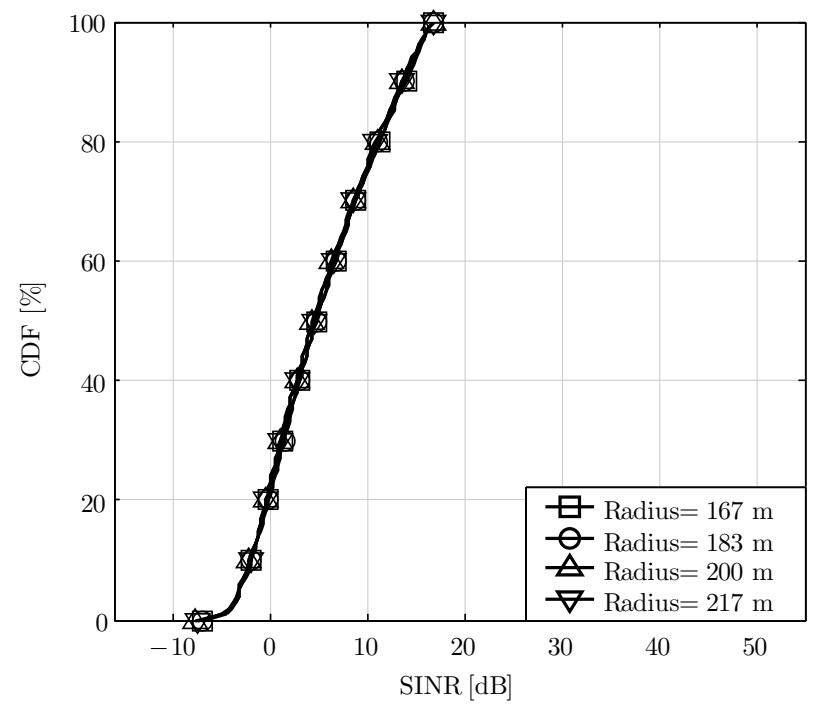

Figure A.4: Received SINR CDF after antenna receiver in a cell of radius 167, 183, 200, and $217 \mathrm{~m}$ in a UMa scenario.

\section{A.2 Link-level simulator}

To measure the performance of the LTE physical layer, a link-level simulation tool has been implemented. The program starts reading a file that configures the general simulation conditions and generates results of Bit Error Rate (BER), BLock Error Rate (BLER) or throughput against Signal to Noise 


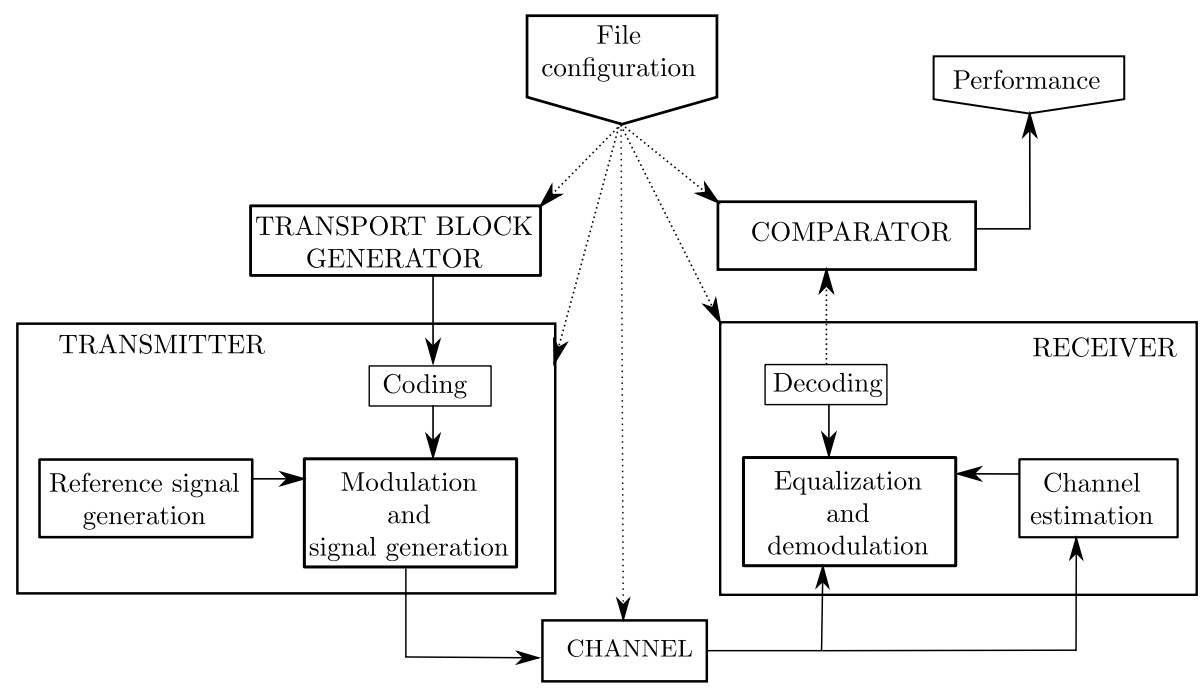

Figure A.5: General description of the LTE link-level simulator.

Ratio (SNR). Figure A.5 shows the general structure of the LTE link-level simulator. The simulator contains five functional blocks: transport block generator, transmitter, channel, receiver, and a comparator. These blocks use a configuration file with the parameters that define the simulation. Finally, performance results are turned into an output file.

In a simulation cycle, one or two transport blocks are created by the transport block generator, processed at the transmitter, sent through the channel, processed by the receiver, and the result is compared with the original transport block. Through this comparison, the number of erroneous bits/blocks can be calculated.

\section{A.2.1 Inputs and outputs}

The input of the simulator corresponds with a parameter file that determines the simulation scenario. Table A.2 shows an example of the possible values of the input data. Running the simulation and measuring its outputs gives an insight into the performance of the whole system depending on the specified operating conditions. When referring to link level, performance is assessed by running Monte Carlo simulations and the typical indicators that are taken as a measure of the system performance are the following: 
- BER, representing the ratio of the number of bits incorrectly received to the total number of bits sent during a specified time interval, versus the correspondent value of SINR. This BER can be measured at the Medium Access Control (MAC) layer and, hence, includes the application of Forward Error Correction (FEC) and Hybrid Automatic RepeatreQuest (HARQ) processes;

- BLER, representing the ratio of the number of Transport Blocks (TBs) incorrectly received to the total number of TBs sent during a specified time interval, versus the correspondent value of SINR;

- Throughput, representing the average data rate that the single transmitter-receiver link is able to deliver, versus the correspondent value of SINR;

- Relative throughput, which normalizes the throughput indicator dividing it by the maximum;

- Link spectral efficiency value, calculated as the throughput normalized by channel bandwidth.

\section{A.2.2 Transport block generator and comparator}

In each simulation cycle, that is, each Transmission Time Interval (TTI), the transport block generator checks the positive ACKnowledgement (ACK)/Negative ACKnowledgement (NACK) bit of the current HARQ process. If the bit is NACK, the same block is sent to the receiver provided the maximum number of transmissions has not been reached. Otherwise, if the bit is ACK, a new block is generated. It is worth noting that, in Multiple Input Multiple Output (MIMO), two transport blocks are generated. Figure A.6 illustrates the general diagram of the transport block generator and comparator. The set of bits is randomly generated through a Primer Modulus Multiplicative Linear Congruential Generation (PMMLCG) algorithm [86]. This generation random method provides one hundred different seeds. After passing through the transmitter, the channel, and the receiver, the block(s) is/are compared with the original version(s), and the comparator calculates the BER or BLER.

\section{A.2.3 Transmitter}

As shown in Figure A.5, the transmitter is composed of three functional blocks: coding, reference signal generation and modulation, and signal generation. A more detailed scheme of the transmitter is given in Figure A.7 and described in the next sections. 
Table A.2: LTE downlink physical layer parameters.

\begin{tabular}{|l|l|l|}
\hline BLOCKs & PARAMETERS & VALUES \\
\hline \hline \multirow{5}{*}{ General } & Carrier frequency & $2 \mathrm{GHz}$ \\
& Subcarrier spacing & $7.5,15 \mathrm{kHz}$ \\
& Number of resource blocks & $1-110$ \\
& FFT size & $128-2048$ \\
& Cyclic prefix type & normal, extended \\
& Antenna scheme & SISO, SIMO, MIMO \\
& Number of HARQ processes & $5,6,7$ \\
& Number of transmissions & $1,2,4$ \\
\hline \multirow{5}{*}{ Transport format } & Transport block size & {$[85]$} \\
& Modulation & QPSK, 16QAM, 64QAM \\
& Mapping antenna type & TD, SM ${ }^{1}$ \\
\hline \multirow{3}{*}{ Channel } & Channel type & AWGN, Rayleigh \\
& Speed [km/h] & $3,50,120, \ldots$ \\
\hline \multirow{3}{*}{ Receiver } & SNR [dB] & $0,1,2, \ldots$ \\
& Receiver type & MRC, MMSE \\
& Channel estimation & Ideal, Linear, MMSE \\
& Decoder type & MAP, log-MAP \\
\hline
\end{tabular}

1: TD: Transmit Diversity, SM: Spatial Multiplexing..

\section{Coding}

The functional blocks that constitute the coding are described in the specification [87]. The first block in the coding stage is composed of the Cyclic Redundancy Check (CRC). This method generates and adds at the end of the original sequence a fixed number of bits based on the information bits and a generator polynomial. CRC stage enables the detection of errors in the communication systems. If the number of bits of the transport block plus the number of CRC bits exceeds 6144 bits, the code block is segmented in smaller blocks. For each segment, a new CRC is calculated and appended.

The most important blocks are coding and rate matching. The internal structure of these blocks is shown in Figure A.8. The transport channel DL$\mathrm{SCH}$ is coded through a rate- $1 / 3$ turbo encoder composed of two recursive systematic convolutional coders and an internal interleaver. After the channel coding and the rate matching, the bit rate is adapted utilizing pruning. 


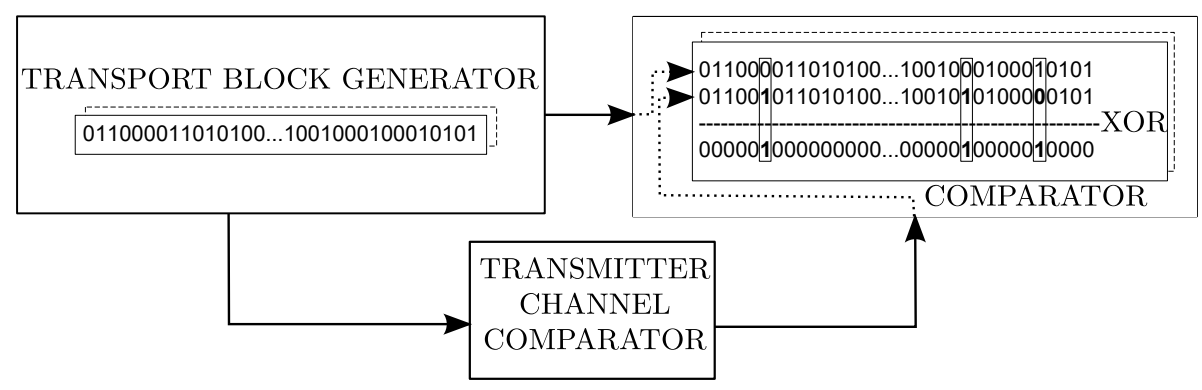

Figure A.6: Functional blocks of the transport block generator and comparator.

Both coders are convolutional coders with feedback, defined by the generator polynomials $g_{0}(x)=1+x^{2}+x^{3}$ and $g_{1}(x)=1+x+x^{3}$. The encoders start in the zero state when they receive the first information bit. At the end of the sequence, the two coders can be in any of the eight states. Normally, each coder will be in a different state since the input of the second coder is an interleaved version of the output of the first coder. Finally, there exist a trellis termination mechanism that causes that both coders return to the zero state. This termination bits are added to the coded sequence and will be used to enhance the performance of the decoding process.

The information and parity bits are separately interleaved and multiplexed into a circular buffer. Knowing the number of scheduled resources, an exact number of bits is extracted from the buffer. This operation is known as bit collection and pruning. After the rate matching the different coded blocks are concatenated.

\section{Modulation and signal generation}

The functional blocks that implement the modulation and the signal generation are [87]:

- Scrambling. Each codeword is scrambled by a sequence that depends on the cell Radio Network Temporary Identifier (RNTI), the time slot, and the cell identity.

- Modulation. The block of scrambled bits is modulated using three possible constellations, QPSK, 16QAM, and 64QAM. The modulation is selected by the scheduler, located in the MAC layer.

- Layer mapping. The antenna mapping is divided into two steps: layer mapping and precoding. The former consists in demultiplexing the mod- 


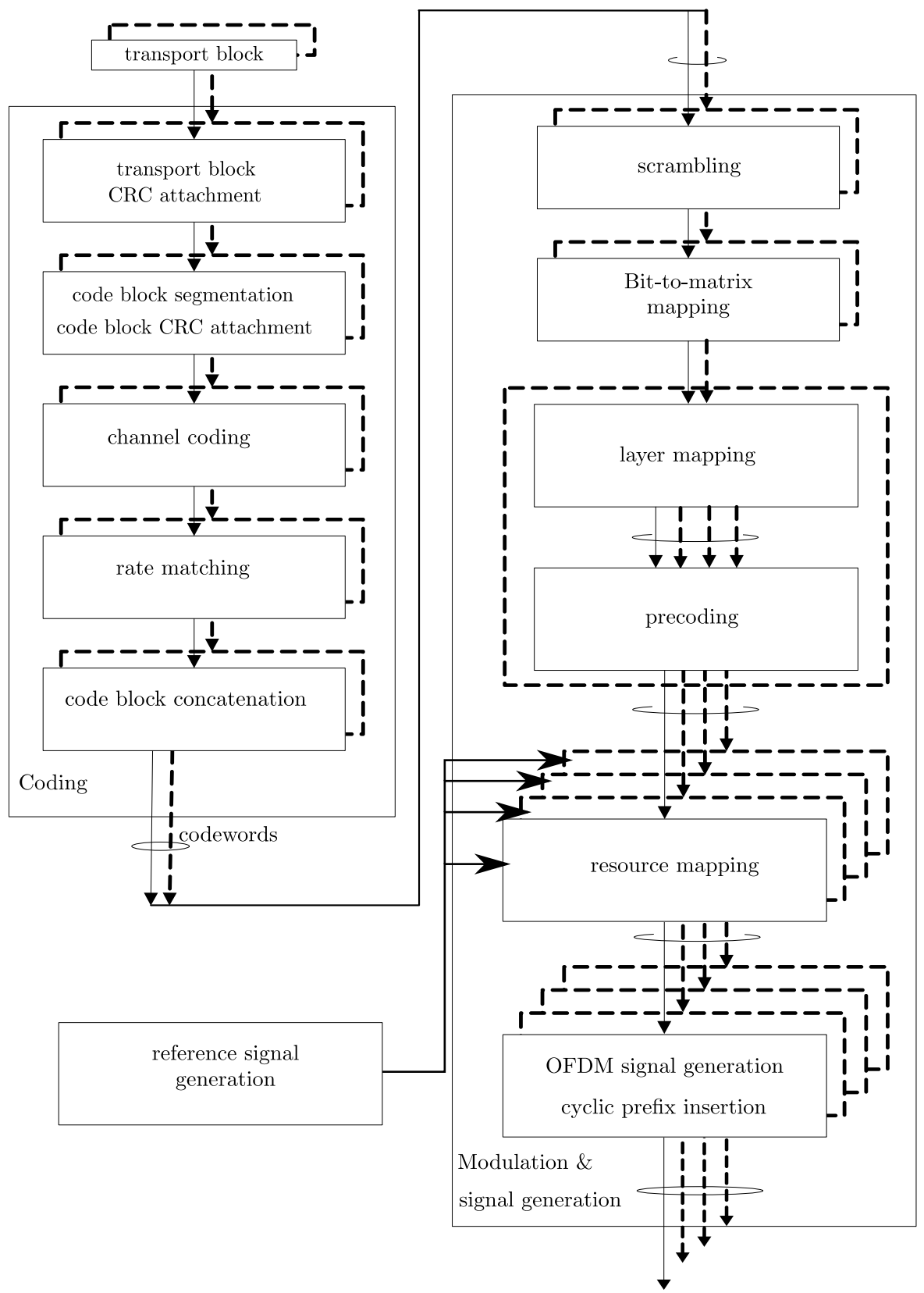

Figure A.7: Processing diagram of the downlink transmitter. 


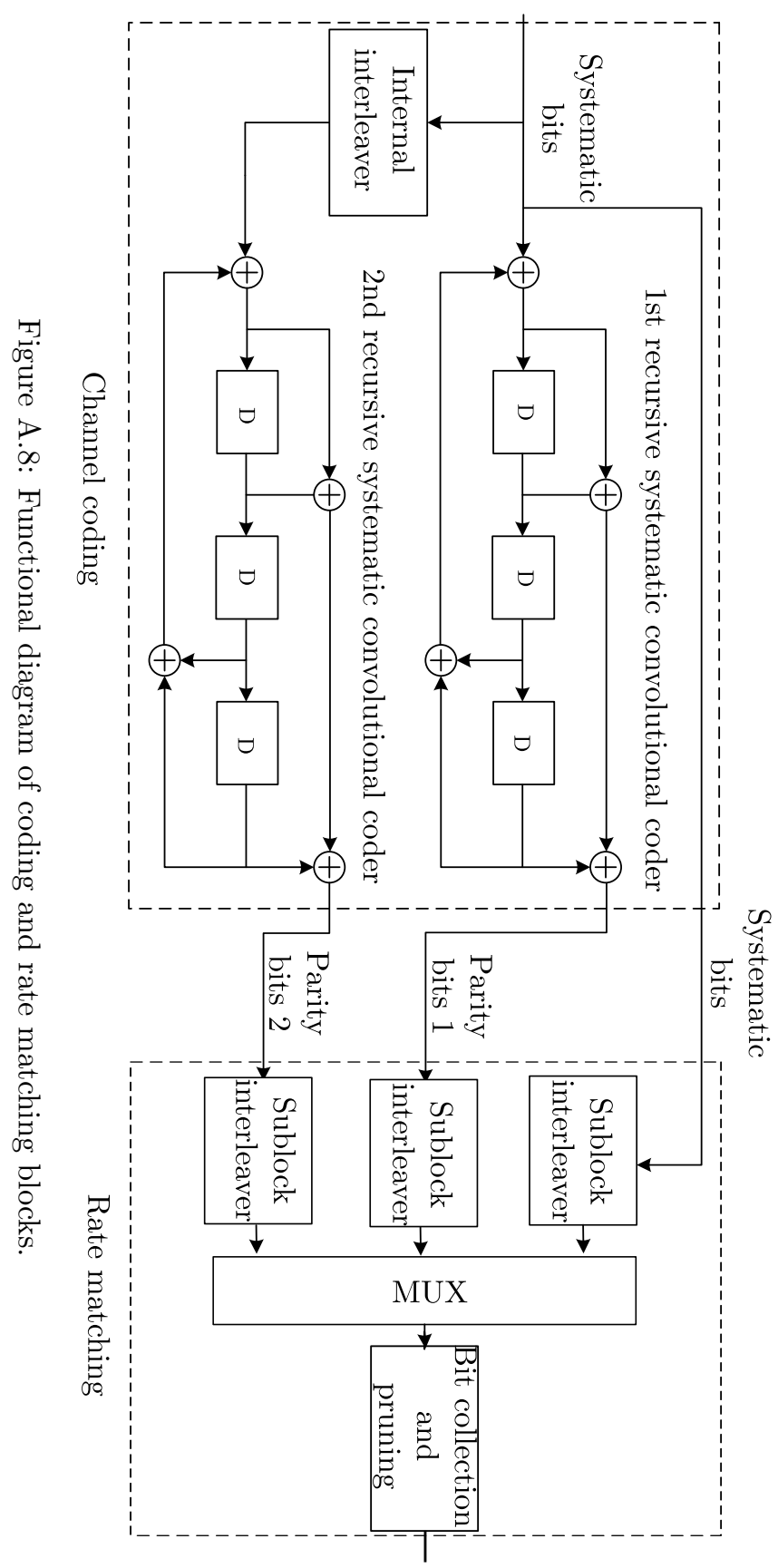


ulated symbols into one or more layers. The latter extracts one symbol of each layer such that these symbols are firstly jointly processed and transmitted through different antennas. There are different configurations for the antenna mapping and precoding configurations that lead to three types of transmission scheme: single-antenna transmission, transmit diversity, and spatial multiplexing.

- Resource mapping. The generated symbols are mapped in the time/frequency grid. Since the LTE system is a coherent system, not all Resource Elements (REs) are available for data. Thus, some REs are allocated for pilots or control signaling. Since the receiver knows where each signal is located in the grid, data can be easily extracted.

- Generation of the OFDM symbol. The generation of complex-valued time-domain Orthogonal Frequency Division Multiplexing (OFDM) signal is performed on each antenna port through an Inverse Fast Fourier Transform (IFFT) function as illustrated in Figure A.9. Finally, a cyclic prefix is added to the signal in the time domain to avoid the symbol and subcarrier interference.

\section{Reference signal generation}

To measure the channel quality, reference signals are inserted into the timefrequency grid. These reference symbols are known both by the transmitter and the receiver. Their generation and the position on the resource grid is specified in [88]. In the simulator, the cell specific reference signals are implemented, whose generation is based on the slot number, the OFDM symbol number within the slot, and the physical layer cell identity.

\section{A.2.4 Channel}

In all mobile communication systems, both the User Equipment (UE) and the BS are surrounded by obstacles such as buildings, cars, mountains, etc. Due to these obstacles, the transmitted signals undergo reflections and diffractions that cause that the UE receives numerous contributions of the transmitted signal. This phenomenon is known as multipath propagation. The received signal is the sum the multiples contributions, each of which will have experienced an attenuation, a phase shift, and a time delay, and its level can change due to the mobility of the UE and the obstacles. This effect is known as fading.

The objective of this section is to model and simulate a time-varying channel between the transmitter and the receiver. The selected methods are based 


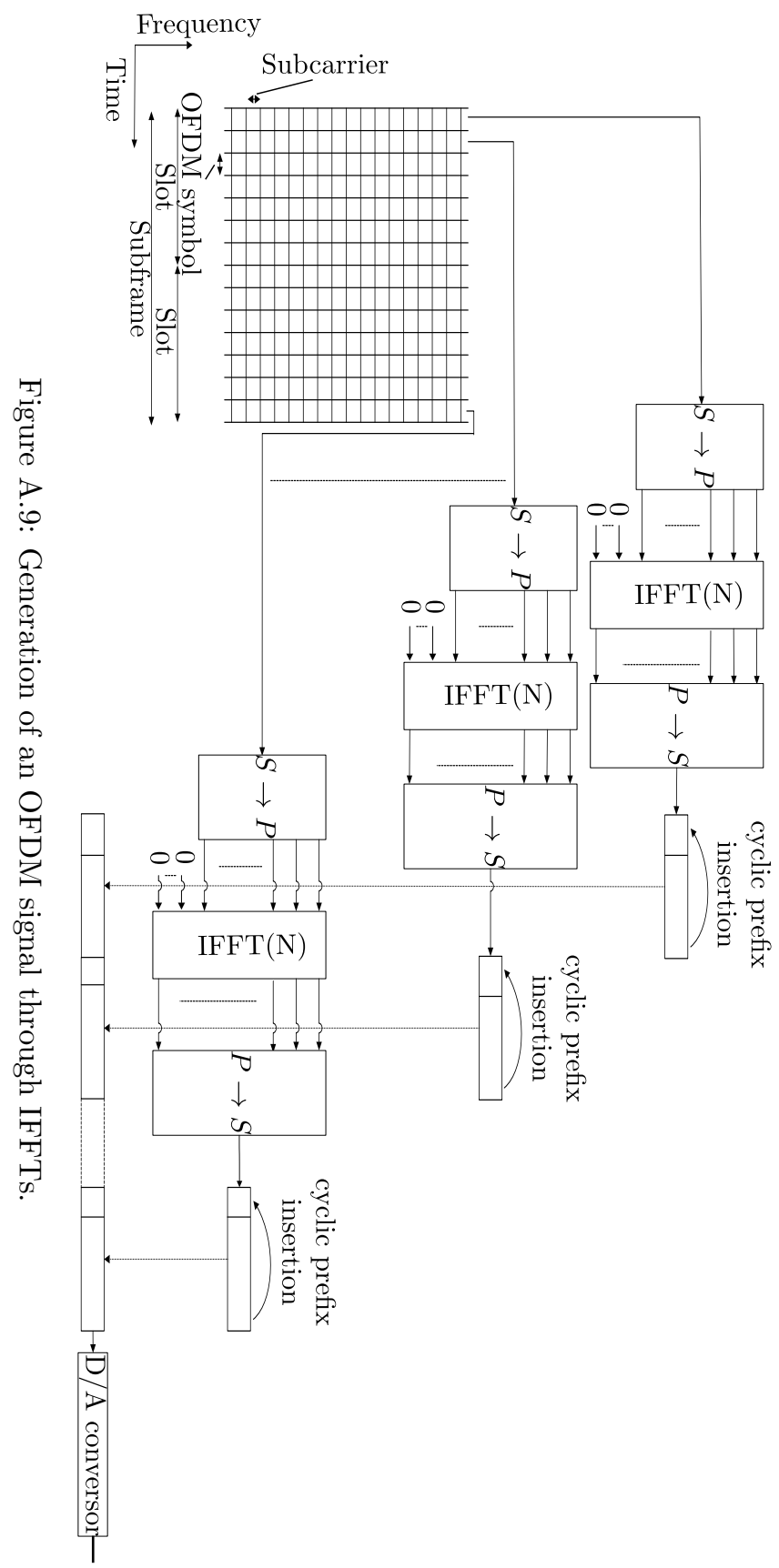




\section{A.2 Link-level simulator}

on statistic models since a deterministic model entails knowledge of antenna configuration, orientation, etc.

\section{Continuous SISO channel model}

There exist numerous methods that model the multipath effect. Figure A.10 illustrates an example of the multipath channel modeled with $L$ ellipses or also known as multipath components. In each ellipse, there are $K$ contributions arriving at the receiver with the same delay $\tau_{l}$. Each contribution is a time shifted replica of the transmitted signal, $s(t)$, with an altered amplitude and phase, which are represented by $h_{l k}$. The received signal in this model can be expressed as

$$
r(t)=\sum_{l=0}^{L-1} \sum_{k=0}^{K-1} h_{l k} s\left(t-\tau_{l}\right) .
$$

Since $\tau_{l}$ is the same for all contributions within each ellipse, the $K$ can be taken together and represented by a factor $h_{l}(t)$ that varies with time. Thus, the received signal can be rewritten as

$$
r(t)=\sum_{l=0}^{L-1} s\left(t-\tau_{l}\right) \sum_{k=0}^{K-1} h_{l k}=\sum_{l=0}^{L-1} s\left(t-\tau_{l}\right) h_{l}(t) .
$$

From Equation( A.3), an expression of the channel can be derived, resulting in a linear time-varying filter with impulse response:

$$
h(t, \tau)=\sum_{l=0}^{L-1} h_{l}(t) \delta\left(\tau-\tau_{l}\right) .
$$

Assuming uncorrelated, isotropic scatterers and omnidirectional receiver antennas, the spectral density of each $h_{l}(t)$ coincides with a classic Doppler spectrum. The speed of the mobile station determines the maximum Doppler frequency, which is inversely related to the coherence time of the multipath component. Furthermore, if there is no Line of Sight (LoS), the modulus of each $h_{l}(t)$ has a Rayleigh distribution. This type of channel is therefore often referred to as Rayleigh fading channel.

In addition to the Doppler spectrum, the channel is completely determined by its discretized Power Delay Profile (PDP), that is to say, the number of multipath components considered, their delay, and the mean relative power assigned to every component. Different profiles have been proposed by the Third Generation Partnership Project (3GPP), depending on the environment [78]. 


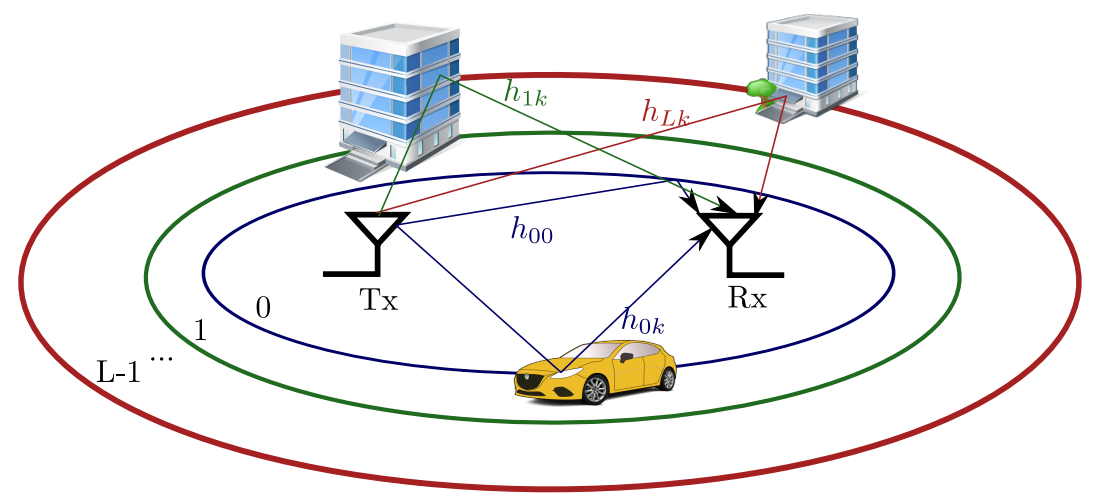

Figure A.10: Example of multipath channel model.

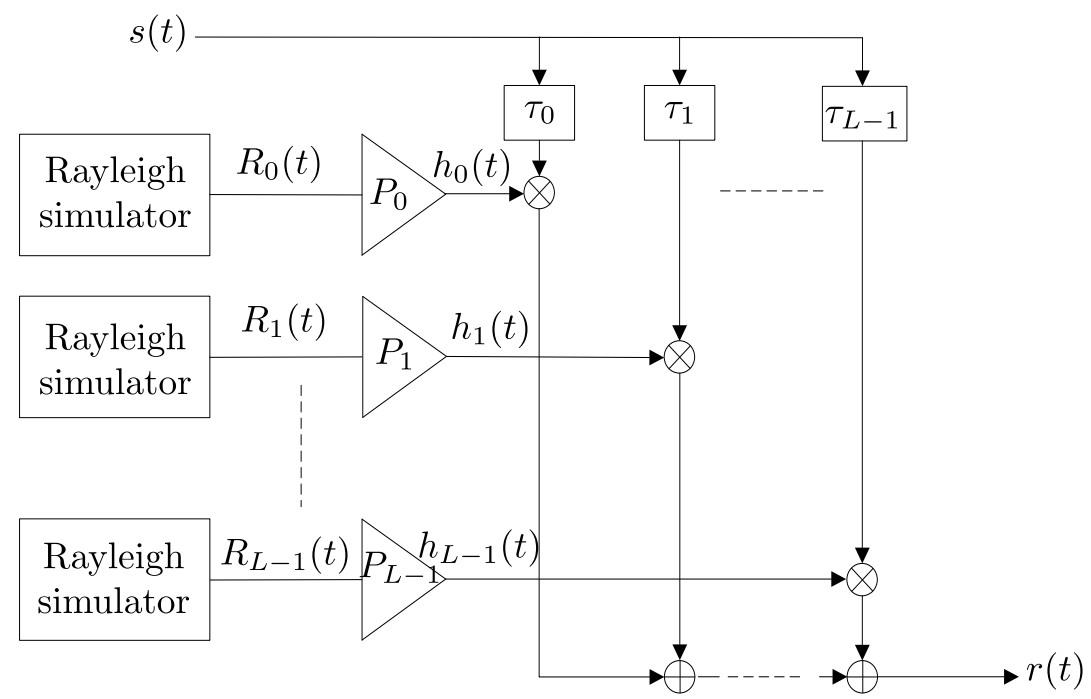

Figure A.11: Implementation of the channel as FIR filter.

The channel can be implemented as a Finite Impulse Response (FIR) filter as shown in Figure A.11. The values of $\tau_{l}$ are the delays of the different multipath components. The coefficients $P_{l}$ determine the mean power of every tap and the $R_{l}(t)$ signals implement the Rayleigh fading process, with unit power and a time correlation according to the maximum Doppler frequency.

In the simulation tool, the Rayleigh fading sequences have been implemented according to two approaches: 
- Filtering method. This statistic method adjusts the spectral density of Gaussian processes using filters. Considering an ellipse $l$ with $K$ contributions and $K$ goes to infinity, $h_{l}(t)$ can be expressed as

$$
h_{l}(t)=\lim _{K \rightarrow \infty} \sum_{k=0}^{K-1} h_{l k}=h_{l, R}(t)+j h_{l, I}(t),
$$

where $h_{l, R}(t)$ and $h_{l, I}(t)$ are random Gaussian variables. Therefore, this problem reduces to generating two Gaussian variables with the desired Doppler spectrum. The spectral density of the Doppler spectrum is generated when passing the signals with normal distribution through a filter with frequency response $\sqrt{S(f)}$ (see Figure A.12). In case of isotropic scattering and omnidirectional receiver antennas, this response has the form of the classic Doppler spectrum

$$
S(f)=\frac{1}{\sqrt{1-\left(\frac{f}{f_{d}}\right)^{2}}} .
$$

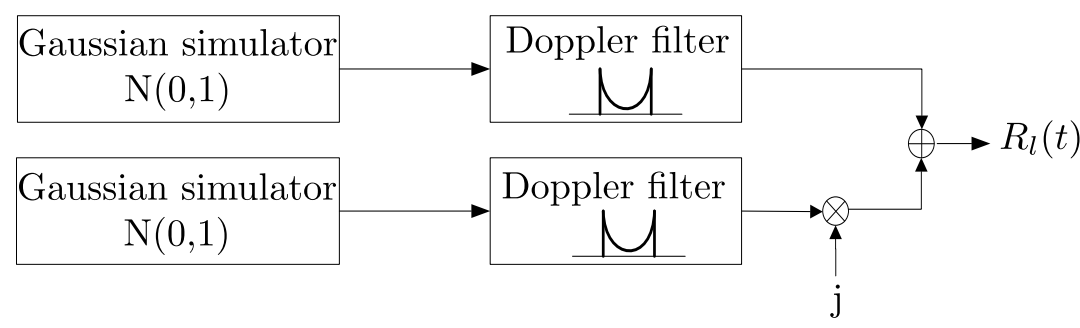

Figure A.12: Generation of a Rayleigh fading signal with classic Doppler spectrum.

- Sum-of-sinudoids method. In [70], a new simulation model is proposed for Rayleigh fading channels. This model is an improved version of the Jakes' model and can be directly used to generate uncorrelated multipath components. The expression of the model is as follows:

$$
\begin{aligned}
R_{l}(t)=\sqrt{\frac{1}{N_{s}}} & \sum_{n=1}^{N_{s}} \cos \left[\omega_{d} t \cos \left(\frac{2 \pi n-\pi+\theta_{l}}{4 N_{s}}\right)+\phi_{n, l}\right] \\
& +j \sqrt{\frac{1}{N_{s}}} \sum_{n=1}^{N_{s}} \cos \left[\omega_{d} t \sin \left(\frac{2 \pi n-\pi+\theta_{l}}{4 N_{s}}\right)+\psi_{n, l}\right]
\end{aligned}
$$




\section{CHAPTER A. SIMULATION TOOLS}

where $\theta_{l}, \phi_{n, l}$ and $\psi_{n, l}$ are mutually independent and uniformly distributed on $[-\pi, \pi)$ for all $n$ and $l$. Here $N_{s}$ stands for the number of sinusoids. Good results are shown, even if the number of sinusoids is as small as a single-digit integer.

\section{Discrete SISO channel model}

At the software level, it is not possible to work with continuous signals, and hence, the models detailed in the previous sections have to be adjusted. The expression for the discrete Single Input Single Output (SISO) channel is

$$
h(k, s)=\sum_{l=0}^{L-1} h_{l}(k) \delta\left(s-s_{l}\right)
$$

where $k=\frac{t}{\Delta t}$ and $s=\frac{\tau}{\Delta \tau}$, with $\Delta t=\Delta \tau$ the time step for the simulation and with $s_{l}$ the time delay of the $l$-th multipath component expressed in discrete time steps. Thus, to generate a discrete SISO channel, $L$ discrete sequences $h_{l}(k)$, each representing a multipath component, have to be generated.

This channel can be easily implemented having stored the values $h_{l}(k)$ for each multipath component $l$ and for each discrete step $k$, and having a buffer with input samples with a length of $L$, that is updated every time instance $k$. Hence, it is necessary that the sampling rate of the channel coincides with the sampling rate of the signal. An adaptation of the PDP can be required, which can be done following the indications of the technical report [89] of 3GPP.

\section{Discrete MIMO channel}

MIMO channels have spatial correlation. In the simplified models of $3 \mathrm{GPP}$, the correlation matrix for each path $l$, is derived as the Kronecker product between the transmitter correlation matrix, $\mathbf{R}^{T x}(l)$ and the receiver correlation matrix, $\mathbf{R}^{R x}(l)$,

$$
\mathbf{R}(l)=\mathbf{R}^{T x}(l) \otimes \mathbf{R}^{R x}(l),
$$

with

$$
\mathbf{R}^{T x}(l)=\left(\begin{array}{ccc}
\rho_{11}^{T x} & \cdots & \rho_{1 M}^{T x} \\
\vdots & \ddots & \vdots \\
\rho_{M 1}^{T x} & \cdots & \rho_{M M}^{T x}
\end{array}\right) \quad \mathbf{R}^{R x}(l)=\left(\begin{array}{ccc}
\rho_{11}^{R x} & \cdots & \rho_{1 N}^{R x} \\
\vdots & \ddots & \vdots \\
\rho_{N 1}^{R x} & \cdots & \rho_{N N}^{R x}
\end{array}\right)
$$

The correlated channels, $h_{m n}(k, s)$ between the $m$-th transmitter antenna and the $n$-th receiver antenna can be calculated by means of the Cholesky decomposition of $\mathbf{R}(l)$ as follows [90]: 


\section{A.2 Link-level simulator}

- Obtain $M N$ SISO uncorrelated channels, $h_{m n}^{\prime}(k, s)$.

- Omitting by simplicity the term $(k, s)$ of $h_{m n}^{\prime}(k, s)$, a column vector $\mathbf{h}^{\prime}$ is created as

$$
\mathbf{h}^{\prime}=\left[h_{00}^{\prime}, h_{01}^{\prime}, \ldots, h_{0 N}^{\prime}, h_{10}^{\prime}, \ldots, h_{1 N}^{\prime}, \ldots h_{M N}^{\prime}\right]^{T} .
$$

- The Cholesky decomposition is calculated as

$$
R(l)=C(l) C(l)^{\mathrm{H}} .
$$

- The vector $\mathbf{h}^{\prime}$ is multiplied by $C(l)$ to obtain the correlated channel sequences, as $\hat{\mathbf{h}}=C(l) \mathbf{h}^{\prime}$.

- The correlated channels $h_{m n}(k, s)$ are obtained from $\hat{\mathbf{h}}$ as

$$
\hat{\mathbf{h}}=\left[h_{00}, h_{01}, \ldots, h_{0 N}, h_{10}, \ldots, h_{1 N}, \ldots h_{M N}\right]^{T} .
$$

The MIMO channel correlation matrices apply for antenna configuration using uniform linear arrays at both evolved Node B (eNB) and UE. The correlation matrices of the eNB with one, two, and four antennas are:

$\mathbf{R}^{T x}=1, \quad \mathbf{R}^{T x}=\left(\begin{array}{cc}1 & \alpha \\ \alpha^{*} & 1\end{array}\right), \quad \mathbf{R}^{T x}=\left(\begin{array}{cccc}1 & \alpha^{1 / 9} & \alpha^{4 / 9} & \alpha \\ \alpha^{1 / 9^{*}} & 1 & \alpha^{1 / 9} & \alpha^{4 / 9} \\ \alpha^{4 / 9^{*}} & \alpha^{1 / 9^{*}} & 1 & \alpha^{1 / 9} \\ \alpha^{*} & \alpha^{4 / 9^{*}} & \alpha^{1 / 9^{*}} & 1\end{array}\right)$,

respectively. The correlation matrices of the UE with one, two, and four antennas are:

$\mathbf{R}^{R x}=1, \quad \mathbf{R}^{R x}=\left(\begin{array}{cc}1 & \beta \\ \beta^{*} & 1\end{array}\right), \quad \mathbf{R}^{R x}=\left(\begin{array}{cccc}1 & \beta^{1 / 9} & \beta^{4 / 9} & \beta \\ \beta^{1 / 9^{*}} & 1 & \beta^{1 / 9} & \beta^{4 / 9} \\ \beta^{4 / 9^{*}} & \beta^{1 / 9^{*}} & 1 & \beta^{1 / 9} \\ \beta^{*} & \beta^{4 / 9^{*}} & \beta^{1 / 9^{*}} & 1\end{array}\right)$,

respectively. The parameters $\alpha$ and $\beta$ set the correlation level (low, medium, and high) according to Table A.3.

\section{A.2.5 Receiver}

As shown in Figure A.5, the receiver is composed of three main blocks: channel estimation, equalization and demodulation, and decoding. Figure A.13 illustrates a more detailed scheme of the receiver. 


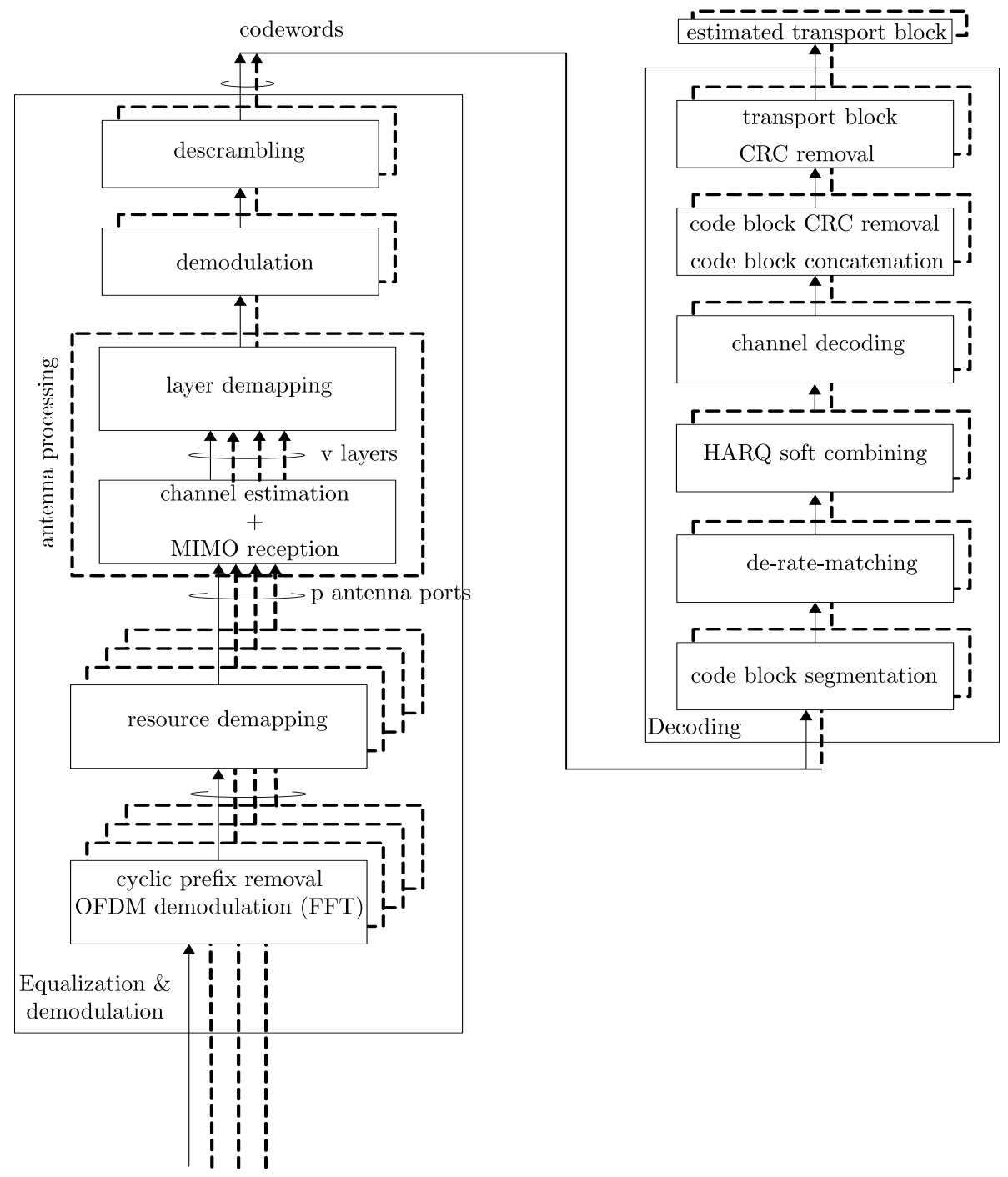

Figure A.13: Functional diagram of the receiver. 


\section{Channel estimation}

At the receiver, the transmitted symbols undergo phase and amplitude shifts due to the multipath effect and the channel variability. To solve these unknown phase and amplitude shifts, two methods are identified: coherent detection and non-coherent detection. The former sends a set of pilots to estimate the channel at the expense of reducing the number of resources for data. The latter does not need to send any pilot symbol.

There exists a large number of methods proposed in the literature. The algorithms implemented in this simulation tool are [79]:

- Semi-ideal channel estimation The most exact channel estimation method that has been implemented is the semi-ideal channel estimator. The name of this method is due to that it is assumed that the channel does not vary in an OFDM symbol, that is to say, the channel is slowfading within the OFDM symbol. The wireless channels, however, can be fast fading depending on the terminals' speed. Thus, the performance of this channel estimation will be degraded on a real channel. However, it is expected that the errors are relatively small due to the short duration of the OFDM symbol.

The method consists in using directly the channel samples. It is worth noting that this method never could be implemented in reality since the channel is not known in advance. However, it is useful to compare the performance of the different channel estimators.

The channel can be modeled by a set of taps whose amplitude is timevarying with a Rayleigh distribution [70]. In each symbol time, a Fast Fourier Transform (FFT) is applied to the set of taps.

- Linear interpolation method This method is the easiest method to estimate the channel through the reference signals. The method consists in calculating the complex channel values $H(l, j)$ at the $(l, j)$-th position of the time-frequency grid. The complex values are calculated by dividing the received signal $Y(l, j)$ by the known transmitted values $X(l, j)$. The other complex channel values are interpolated by linear interpolation,

Table A.3: Parameters of the correlation matrices.

\begin{tabular}{|c|c|c|c|c|c|}
\hline \multicolumn{2}{|c|}{ Low } & \multicolumn{2}{c|}{ Medium } & \multicolumn{2}{c|}{ High } \\
\hline \hline$\alpha$ & $\beta$ & $\alpha$ & $\beta$ & $\alpha$ & $\beta$ \\
\hline 0 & 0 & 0.3 & 0.9 & 0.9 & 0.9 \\
\hline
\end{tabular}


first in the frequency dimension, then in the time dimension. Obviously, this method is not very efficient, but it is appealing due to its simplicity.

- Linear MMSE The Wiener filter or Linear Minimum Mean Square Error (LMMSE) uses the knowledge of the channel features such as the spaced-frequency and spaced-time correlation functions to estimate the channel in positions of the time-frequency grid where there are not pilots [91]. There exist different methods in the literature to implement Wiener filters. On the one hand, 2-Dimensions (2D) filters take jointly into consideration the channel in frequency and time dimensions. However, these methods are rather complicated, and they are often simplified by using two 1-Dimension (1D) filtering, firstly, in the frequency dimension, then in the time dimension.

The Wiener filtering aims at minimizing the mean quadratic error between the actual channel and the estimated channel. The estimate is calculated through the following equation [91]:

$$
\mathbf{H}_{L M M S E}=\mathbf{c}^{\mathrm{H}} \hat{\mathbf{H}},
$$

where $\hat{\mathbf{H}}$ are the channel estimates, and $\mathbf{c}$ are the coefficients of the filter, whose expression is

$$
\mathbf{c}=\left(\mathbf{R}_{h}+\sigma^{2} \mathbf{I}\right)^{-1} \mathbf{r},
$$

where $\mathbf{R}_{h}$ is the auto-correlation matrix of the reference signals, $\mathbf{r}$ is the cross-correlation matrix between the channel and the reference signals, and $\sigma^{2}$ is the noise variance of the channel. The auto-correlation and cross-correlation matrices can be expressed as follows:

$$
\begin{array}{r}
\mathbf{R}_{h}=\left[\begin{array}{cccc}
E\left[\hat{\mathbf{H}}_{0} \hat{\mathbf{H}}_{0}^{\mathrm{H}}\right] & \left.E \hat{\mathbf{H}}_{0} \hat{\mathbf{H}}_{p}^{\mathrm{H}}\right] & \cdots & E\left[\hat{\mathbf{H}}_{0} \hat{\mathbf{H}}_{p\left(N_{p}-1\right)}^{\mathrm{H}}\right] \\
E\left[\hat{\mathbf{H}}_{p} \hat{\mathbf{H}}_{0}^{\mathrm{H}}\right] & E\left[\hat{\mathbf{H}}_{p} \hat{\mathbf{H}}_{p}^{\mathrm{H}}\right] & \cdots & E\left[\hat{\mathbf{H}}_{p} \hat{\mathbf{H}}_{p\left(N_{p}-1\right)}^{\mathrm{H}}\right] \\
\vdots & \vdots & \ddots & \vdots \\
E\left[\hat{\mathbf{H}}_{p\left(N_{p}-1\right)}^{\mathrm{H}} \hat{\mathbf{H}}_{0}\right] & E\left[\hat{\mathbf{H}}_{p\left(N_{p}-1\right)}^{\mathrm{H}} \hat{\mathbf{H}}_{p}\right] & \cdots & E\left[\hat{\mathbf{H}}_{p\left(N_{p}-1\right)}^{\mathrm{H}} \hat{\mathbf{H}}_{p\left(N_{p}-1\right)}\right]
\end{array}\right], \\
\mathbf{r}=\left[\begin{array}{c}
E\left[\hat{\mathbf{H}}_{0} \hat{\mathbf{H}}_{k}^{\mathrm{H}}\right] \\
E\left[\hat{\mathbf{H}}_{p} \hat{\mathbf{H}}_{k}^{\mathrm{H}}\right] \\
\vdots \\
E\left[\hat{\mathbf{H}}_{p\left(N_{p}-1\right)}^{\mathrm{H}} \hat{\mathbf{H}}_{k}\right]
\end{array}\right],
\end{array}
$$

where $\hat{\mathbf{H}}_{p i}$ is the estimated channel at the $p i$-th pilot location, and $\hat{\mathbf{H}}_{k}$ is the channel at the $k$-th subcarrier. The spaced-frequency and spaced-time 
correlation functions are shown in Equation (4.17) and Equation (4.18), respectively. Concerning the SNR, it is unknown a priori, but it is approximated by the SNR that is set in the input parameters.

- Mixed estimators This section describes two mixed estimators that contain particular features of the previously explained estimators. The first mixed estimator performs a Wiener filtering in frequency dimension and an interpolation in the time dimension. In this way, the complete knowledge of the channel in the time domain is suppressed, and an error is introduced in the channel estimation. The second mixed estimator performs the same channel estimation as the first one, but only the six nearest pilots are taken into consideration in the calculation of the autocorrelation and cross-correlation matrices. Moreover, a linear interpolation is made at the edges of the time-frequency grid.

\section{Equalization and demodulation}

At the receiver, in each OFDM symbol, the cyclic prefix is suppressed, and an FFT is applied to convert the signal into the frequency domain. Due to this conversion, the signal is divided into a time-frequency grid where each resource element is the product between the transmitted signal by the channel response. To obtain the transmitted signal, the received signal is equalized by a filter whose response is the inverse of the estimated channel. This equalization can be problematic if the channel response is low since the noise is amplified.

In Single Input Multiple Output (SIMO) case, each antenna has a copy of the transmitted signal (with the consequent degradation due to the channel). These copies will improve the received signal through different combining methods. In the simulator, the Maximum Ratio Combining (MRC) and the Minimum Mean Square Error (MMSE) methods have been implemented.

Once the channel has been equalized, the Quadrature Amplitude Modulation (QAM) and Phase-Shift Keying (PSK) symbols are demodulated. There exist two demodulation types: hard and soft decision. In the former case, each received complex symbol is transformed to the nearest symbol of the constellation. In the latter case, the received symbols are converted to a sequence of real values, where a negative value represents a 0 -bit, and a positive value represents a 1-bit. This information is used by the decoder to improve performance.

Figure A.14 depicts both demodulation techniques for the Quadrature Phase-Shift Keying (QPSK) demodulation. The received symbol, represented by a black point, is located in the fourth quadrant. If the decision is hard, the received symbol is transformed to 01 . Otherwise, the received symbol is transformed to -0.350 .88 . The first bit corresponds to the sign of the real part and the second bit to the sign of the complex part. 

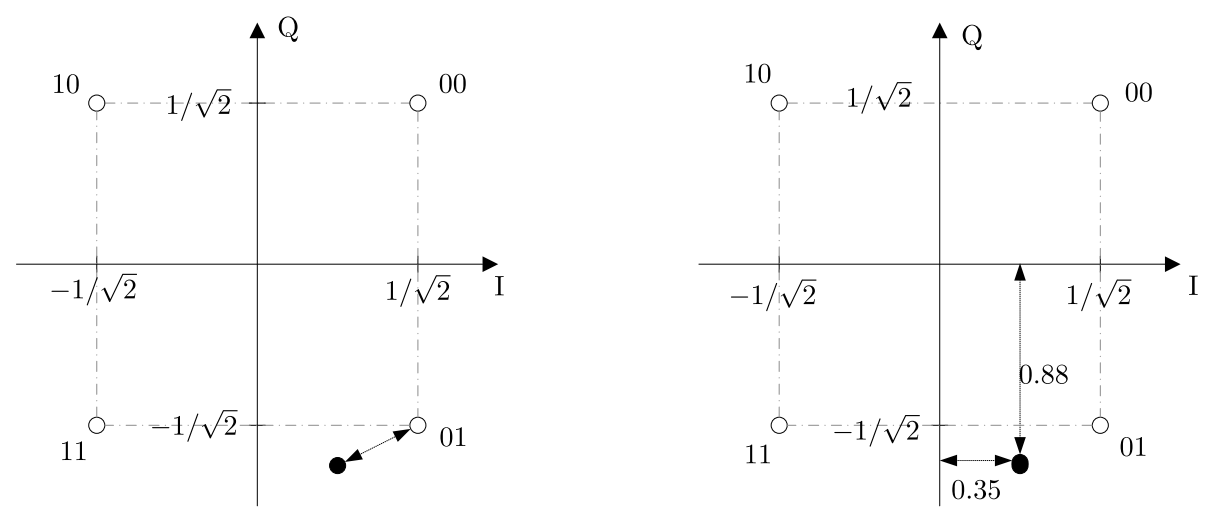

Figure A.14: Example of hard and soft demodulation. (a) Right: hard. (b)

Left: soft.

\section{Decoding}

The structure of the decoder can be found in Figure A.15. The turbo decoder is composed of two component decoders that are linked by an interleaver. The decoder has three inputs: received systematic bits, received parity bits, and apriori information that is originated from the another decoder. Next, a-posteriori information is calculated, which is a soft estimate of the decoded bits, also referred to as soft outputs. Subtracting the systematic bits and the apriori information from the a-posteriori information results in the so-called extrinsic information, which forms the apriori information for the other decoder component.

The soft values are represented in terms of Log-Likelihood Ratios (LLRs), whose sign represents the bit value (negative for 0-bit and positive for 1-bit), and the magnitude represents the probability of a correct decision. The LLR of a data bit $d_{k}$ is denoted by $L\left(d_{k}\right)$, and it is defined as the natural logarithm of the division between the probability of being a 1-bit and the probability of being a 0-bit

$$
L\left(d_{k}\right)=\ln \left(\frac{\operatorname{Prob}\left(d_{k}=1\right)}{\operatorname{Prob}\left(d_{k}=0\right)}\right) .
$$

The soft outputs are represented as the conditional probability of being transmitted a bit $x_{k}$ if the received bit is $y_{k}$

$$
L\left(y_{k} \mid x_{k}\right)=\ln \left(\frac{P\left(y_{k} \mid x_{k}=1\right)}{P\left(y_{k} \mid x_{k}=0\right)}\right) .
$$




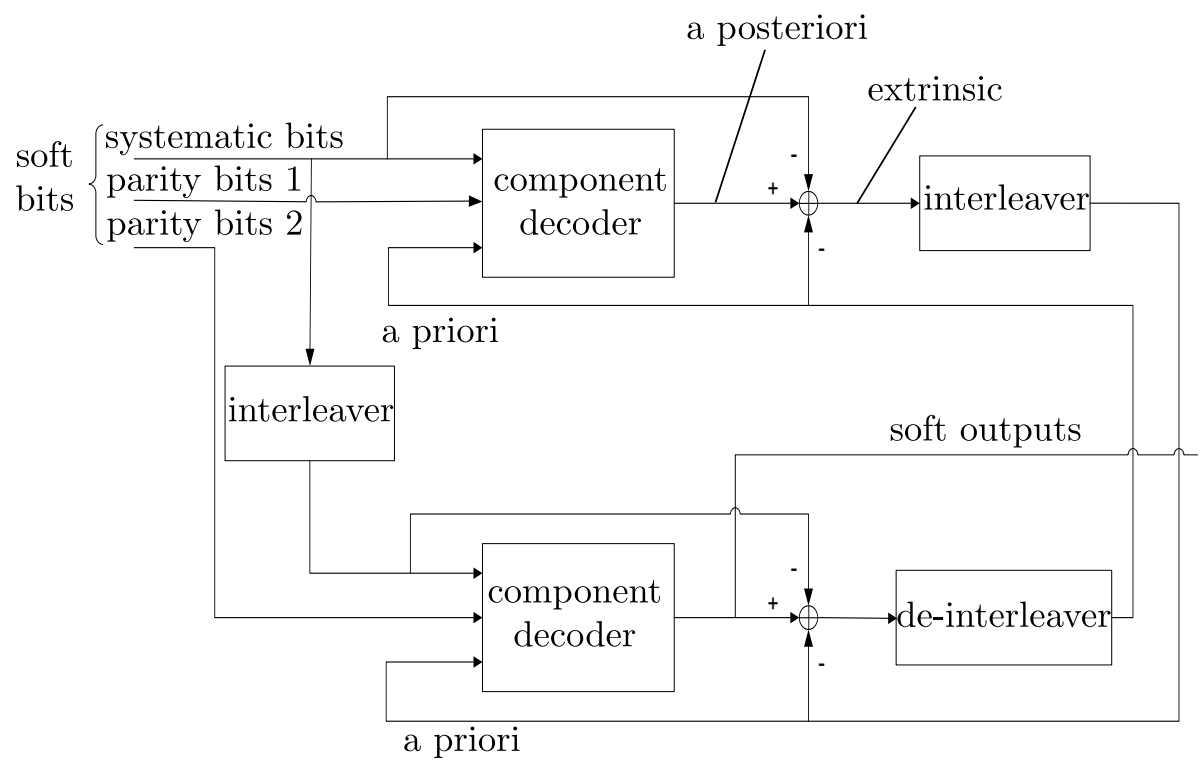

Figure A.15: Functional diagram of the turbo decoder.

In a Gaussian channel with fading of amplitude $a$, noise variance $2 \sigma^{2}$, and a Binary Phase-Shift Keying (BPSK) modulation with energy per bit $E_{b}$, the soft inputs can be expressed as

$$
L\left(y_{k} \mid x_{k}\right)=\ln \left(\frac{e^{\frac{-E_{b}}{2 \sigma^{2}}\left(y_{k}-a\right)^{2}}}{e^{\frac{-E_{b}}{2 \sigma^{2}}\left(y_{k}+a\right)^{2}}}\right)=L_{c} y_{k},
$$

where

$$
L_{c}=4 a \frac{E_{b}}{2 \sigma^{2}} .
$$

The parameter $L_{c}$ is defined as the channel reliability, and it depends on the SNR and the fading amplitude $a$. Hence, to obtain the LLR value for a received bit, it is only multiplied by $L_{c}$, which represents the channel quality experienced by that bit. The better the channel quality, the higher the probability of a correct decision and vice versa.

The decoder operates iteratively, and hence, its name of turbo decoder. In the first iteration, the first component decoder takes only the received values and produces a-posteriori information, which is an estimate of the transmitted bits. Subtracting the systematic bits from the a-posteriori information results in the extrinsic information, which forms apriori information for the second 


\section{CHAPTER A. SIMULATION TOOLS}

component decoder. This decoder uses this information along with the received bits to calculate estimates of the transmitted bits, which forms the soft outputs. In the second iteration, the first component decoder decodes the received bits but now with the additional information provided by the second component decoder in the first iteration. This information enables the first component decoder to obtain more accurate information subsequently used by the second component decoder. This cycle is repeated several times so that with each iteration the error rate decreases. However, the improvement in performance decreases as the number of iterations increases. Hence, for complexity reasons, usually only about eight iterations are used.

There exist two algorithms for the component decoders: Maximum a Posteriori (MAP) and log-MAP algorithms. The former algorithm is optimum but involved. The latter is a simplification of the MAP algorithm, and it offers similar performance with a reasonable reduction of complexity.

The fact of working with soft values facilities the implementation of the HARQ mechanism. The buffer HARQ is located just before the turbo decoder (see Figure A.13). When a transport block is received for the first time, its segments are stored in a buffer and decoded. If the transport block contains errors after the decoding operation, a NACK bit is sent to the transmitter, causing the retransmission of the same packet. The new packets are stored in the same buffer by adding the soft values. In fact, this results in a weighted sum according to the channel quality. This sum is the input of the decoder. This process continues until the CRC checks that there are no errors o until the maximum number of transmissions is reached. If the transport block is composed of several segments, these will be successively decoded. Each segment has an appended CRC such that if any packet has errors, the remaining segments will not be decoded, resulting in a gain of time and resource saving. 


\section{Appendix B}

\section{Basics on non-coherent signaling}

\section{B.1 Basics on subspaces}

We define an M-dimensional subspace of $\mathbb{C}^{T}, \Omega_{\mathbf{X}}$, with $T>M$ as

$$
\Omega_{\mathbf{X}}=\operatorname{Span}\left(\mathbf{x}_{1} \mathbf{x}_{2} \cdots \mathbf{x}_{M}\right)=\operatorname{Span}(\mathbf{X})
$$

where $\Omega_{\mathbf{X}}$ spans all linear combinations of the column vectors $\left\{\mathbf{x}_{1}, \mathbf{x}_{2}, \cdots, \mathbf{x}_{M}\right\}$ of $\mathbf{X}$. If those column vectors are linearly independently, they compose a basis of $\Omega_{\mathbf{X}}$. It is worth noting that the basis $\mathbf{X}$ is not unique. In fact, given any $M \times M$ invertible matrix $\mathbf{A}$, the result of the product of $\mathbf{X}$ and $\mathbf{A}$ is another valid basis [92].

\section{B.2 Optimal signal structure}

The optimal structure of signal that achieves capacity can be written as [31] [32]

$$
\mathbf{X}=\mathbf{\Phi} \mathbf{V}
$$

where $\boldsymbol{\Phi}$ is an isotropically distributed matrix whose columns are orthonormal, i.e., $\boldsymbol{\Phi}^{\mathrm{H}} \boldsymbol{\Phi}=\mathbf{I}_{M}$ and $\mathbf{V}$ is a real, non-negative, and diagonal matrix. An isotropically distribution matrix means that the probability density function is unchanged when the matrix is left-multiplied by any $T \times T$ deterministic unitary matrix. For high SNR and $T>M$ or $T \gg M$, if $\mathbf{V}$ is an identity matrix, this structure achieves capacity. This modulation is known in the literature 
as Unitary Space-Time Modulation (USTM). This modulation is completely specified by $\boldsymbol{\Phi}$, and thus, the directions of the column vectors [31] [32]. However, Zheng and Tse, thanks to a geometric interpretation of the problem, showed in [33] that the information is not contained in the directions but in the subspace $\Omega_{\mathbf{X}}$. The set of $M$-dimensional subspaces of $\mathbb{C}^{T}$ is referred to as Grassmannian manifold.

\section{B.3 Preliminaries of Grassmannian signaling}

The set of all $T \times M$ complex unitary matrices is referred to as the Stiefel manifold, $S(T, M)=\left\{\boldsymbol{\Phi} \in \mathbb{C}^{T \times M}: \boldsymbol{\Phi}^{\mathrm{H}} \boldsymbol{\Phi}=\mathbf{I}_{M}\right\}$. The Grassmann manifold is defined as a quotient space of the Stiefel manifold with respect to an equivalence relation, whereby two unitary matrices $\mathbf{U}$ and $\mathbf{V} \in S(T, M)$, are equivalent if their columns span the same subspace. Each of the $M$-dimensional subspaces corresponds to a point on the Grassmann manifold and can be represented by any of the $T \times M$ unitary matrices spanning it. 


\section{Appendix $\mathrm{C}$}

\section{Signal to noise ratio calculation}

The SNR of the system model of Equation (4.1) for $T$ channel uses can be expressed as

$$
\mathrm{SNR}=\frac{P_{\mathbf{y}}}{P_{\mathbf{z}}}
$$

where the useful (no noise) received signal power is averaged over $T$ channel uses as

$$
\begin{array}{r}
P_{\mathbf{y}}=\frac{1}{T} \sum_{t=1}^{T} \mathbb{E}\left[\operatorname{Tr}\left(\left[\mathbf{x}_{t}^{\dagger} \mathbf{H}_{t} \mathbf{H}_{t}^{\dagger} \mathbf{x}_{t}\right)\right]=\right. \\
\frac{1}{T} \sum_{t=1}^{T} \mathbb{E}\left[\operatorname{Tr}\left(\left[\mathbf{H}_{t} \mathbf{H}_{t}^{\dagger} \mathbf{x}_{t} \mathbf{x}_{t}^{\dagger}\right)\right] .\right.
\end{array}
$$

If the transmitted vectors $\mathbf{x}_{t}$ are independent of the channel $\mathbf{H}_{t}$, and the channel matrix presents a temporal correlation $\boldsymbol{\Gamma}_{\mathbf{H}}$, we have

$$
\begin{array}{r}
P_{\mathbf{y}}=\frac{1}{T} \sum_{t=1}^{T} \operatorname{Tr}\left(\mathbb{E}\left[\mathbf{H}_{t} \mathbf{H}_{t}^{\dagger}\right] \mathbb{E}\left[\mathbf{x}_{t} \mathbf{x}_{t}^{\dagger}\right]\right)=\frac{1}{T} \operatorname{Tr}\left(\boldsymbol{\Gamma}_{\mathbf{H}} \sum_{t=1}^{T} \mathbb{E}\left[\mathbf{x}_{t} \mathbf{x}_{t}^{\dagger}\right]\right)= \\
\frac{1}{T} \operatorname{Tr}\left(\boldsymbol{\Gamma}_{\mathbf{H}} \boldsymbol{\Gamma}_{\mathbf{X}}\right)=\frac{1}{T} \operatorname{Tr}\left(\boldsymbol{\Gamma}_{\mathbf{X}} \boldsymbol{\Gamma}_{\mathbf{H}}\right) .
\end{array}
$$


Since noise samples are i.i.d with normal distribution $\mathcal{C N}(0,1)$, the noise average power is

$$
\begin{gathered}
P_{\mathbf{z}}=\frac{1}{T} \sum_{t=1}^{T} \operatorname{Tr}\left(\mathbb{E}\left[\mathbf{w}_{t}^{\dagger} \mathbf{w}_{t}\right]\right)= \\
\frac{1}{T} \sum_{t=1}^{T} \mathbb{E}\left[\operatorname{Tr}\left(\mathbf{w}_{t}^{\dagger} \mathbf{w}_{t}\right)\right]=N .
\end{gathered}
$$

It is straightforward to obtain

$$
\mathrm{SNR}=\frac{P_{\mathbf{y}}}{P_{\mathbf{z}}}=\frac{\operatorname{Tr}\left(\boldsymbol{\Gamma}_{\mathbf{X}} \boldsymbol{\Gamma}_{\mathbf{H}}\right)}{T N}
$$

If the antennas are spatially uncorrelated with i.i.d. fading $\mathcal{C N}(0,1)$, the channel covariance matrix is $\boldsymbol{\Gamma}_{\mathbf{H}}=N \mathbf{I}_{M}$, and the SNR has the following expression:

$$
\mathrm{SNR}=\frac{\operatorname{Tr}\left(\boldsymbol{\Gamma}_{\mathbf{X}}\right)}{T}
$$

Note that for unitary signals, such as Grassmannian signaling, the covariance matrix of the transmitted signal is the identity matrix $\mathbf{I}_{M}$, and the SNR is

$$
\mathrm{SNR}=\frac{\operatorname{Tr}\left(\mathbf{I}_{M}\right)}{T}=\frac{M}{T} .
$$

The Alamouti and Golden codes have a covariance matrix proportional to the identity matrix $\mathbf{I}_{M}$, i.e. $\boldsymbol{\Gamma}_{\mathbf{X}}=P_{\mathbf{X}} \mathbf{I}_{M}$, if the power of all transmitted symbols within the code is the same. For example, the Alamouti code contains two symbols $\left(s_{1}\right.$ and $\left.s_{2}\right)$ of power $P_{A}$. Thus, the covariance matrix of the matrix $\mathbf{X}$ is

$$
\boldsymbol{\Gamma}_{\mathbf{X}}=2 P_{A} \mathbf{I}_{M}
$$

On the other hand, the Golden code contains four symbols $\left(s_{1}, s_{2}, s_{3}\right.$, and $\left.s_{4}\right)$ of power $P_{G}$. In this case, the covariance matrix of the matrix $\mathbf{X}$ is

$$
\boldsymbol{\Gamma}_{\mathbf{X}}=\frac{1}{5}\left(|\alpha|^{2}\left(P_{G}+P_{G} \theta^{2}\right)+|\gamma|^{2}\left(P_{G}+P_{G} \bar{\theta}^{2}\right)\right) \mathbf{I}_{M}
$$




\section{Appendix D}

\section{Derivation details of Section 4.6}

\section{D.1 Derivation of Equation (4.23)}

The covariance matrix of the received signal when the transmitted signal to all users is $\mathbf{B}=\left(\mathbf{B}_{1}, \ldots, \mathbf{B}_{U}\right)$ is defined as:

$$
\begin{aligned}
\boldsymbol{\Sigma}(\mathbf{B}, \rho) & =\mathbb{E}\left[\mathbf{Y}_{u} \mathbf{Y}_{u}^{\mathrm{H}} \mid \mathbf{X}=\mathbf{B}\right] \\
& =\mathbb{E}\left[\left(\left(\sum_{m=1}^{U} \sqrt{P_{t} \gamma_{m}} \mathbf{X}_{\ell}\right) \mathbf{H}_{u}+\mathbf{Z}_{u}\right)\left(\left(\sum_{n=1}^{U} \sqrt{P_{t} \gamma_{n}} \mathbf{X}_{\ell}\right) \mathbf{H}_{u}+\mathbf{Z}_{u}\right)^{\mathrm{H}}\right] \\
& =P_{t} \sum_{m=1}^{U} \sum_{n=1}^{U} \sqrt{\gamma_{m} \gamma_{n}} \mathbf{B}_{m} \mathbb{E}\left[\mathbf{H}_{u} \mathbf{H}_{u}{ }^{\mathrm{H}}\right] \mathbf{B}_{n}^{\mathrm{H}}+\mathbb{E}\left[\mathbf{Z}_{u} \mathbf{Z}_{u}{ }^{\mathrm{H}}\right] \\
& =N P_{t} \sum_{m=1}^{U} \sum_{n=1}^{U} \sqrt{\gamma_{m} \gamma_{n}} \mathbf{B}_{m} \mathbf{B}_{n}^{\mathrm{H}}+N \mathbf{I}_{T},
\end{aligned}
$$

where the channel covariance matrix is $\mathbb{E}\left[\mathbf{H}_{u} \mathbf{H}_{u}{ }^{\mathrm{H}}\right]=N \mathbf{I}_{M}$, and $P_{t}=\frac{\rho T}{\operatorname{Tr}\left(\boldsymbol{\Gamma}_{\mathbf{X}}\right)}$.

\section{D.2 Derivation of Equations (4.27) and (4.28)}

The covariance matrix for $\mathbf{B}=\left(\mathbf{S}_{i}, \mathbf{0}, \ldots, \mathbf{0}\right)$ is 


$$
\begin{aligned}
\boldsymbol{\Sigma}(\mathbf{B}, \rho) & =\mathbb{E}\left[\left(\sqrt{P_{t} \gamma_{i}} \mathbf{S}_{i} \mathbf{H}_{u}+\mathbf{Z}_{u}\right)\left(\sqrt{P_{t} \gamma_{i}} \mathbf{S}_{i} \mathbf{H}_{u}+\mathbf{Z}_{u}\right)^{\mathrm{H}}\right] \\
& =P_{t} \gamma_{i} \mathbf{S}_{i} \mathbb{E}\left[\mathbf{H}_{u} \mathbf{H}_{u}{ }^{\mathrm{H}}\right] \mathbf{S}_{i}{ }^{\mathrm{H}}+N \mathbf{I}_{T} \\
& =N P_{t} \gamma_{i} \mathbf{S}_{i} \mathbf{S}_{i}{ }^{\mathrm{H}}+N \mathbf{I}_{T} .
\end{aligned}
$$

The equivalent detection rule for user 1 can be obtained as follows:

$$
\begin{aligned}
\hat{\imath}_{u, 1} & =\arg \max _{i} G\left(\mathbf{\Upsilon}, \mathbf{B}=\left(\mathbf{S}_{i}, \mathbf{0}, \ldots, \mathbf{0}\right), \rho_{u}^{\prime}\right) \\
& =\arg \max _{i} \frac{\exp \left(-\operatorname{Tr}\left(\mathbf{\Upsilon}^{\mathrm{H}}(\boldsymbol{\Sigma}(\mathbf{B}, \rho))^{-1} \mathbf{\Upsilon}\right)\right)}{\pi^{T N} \operatorname{det}^{N}\left(\boldsymbol{\Sigma}\left(\mathbf{B}, \rho_{u}^{\prime}\right)\right)} \\
& =\arg \max _{i} \frac{\exp \left(-\operatorname{Tr}\left(\mathbf{\Upsilon}^{\mathrm{H}}\left(N P_{t} \gamma_{i} \mathbf{S}_{i} \mathbf{S}_{i}{ }^{\mathrm{H}}+N \mathbf{I}_{T}\right)^{-1} \mathbf{\Upsilon}\right)\right)}{\pi^{T N} \operatorname{det}^{N}\left(N P_{t} \gamma_{i} \mathbf{S}_{i} \mathbf{S}_{i}{ }^{\mathrm{H}}+N \mathbf{I}_{T}\right)}
\end{aligned}
$$

Using the properties $\operatorname{det}(d \mathbf{C})=d^{m} \operatorname{det}(\mathbf{C})$ where $\mathbf{C}$ is an $m \times m$ matrix, $\operatorname{det}\left(\mathbf{I}_{s}+\mathbf{A B}\right)=\operatorname{det}\left(\mathbf{I}_{n}+\mathbf{B A}\right)$, and assuming unitary matrices, i.e. $\mathbf{S}_{i}^{\mathrm{H}} \mathbf{S}_{i}=$ $\mathbf{I}_{M}$, the denominator of Equation (D.3) is simplified to [93]:

$$
\begin{aligned}
\operatorname{det}\left(N \gamma_{i} P_{t} \mathbf{S}_{i} \mathbf{S}_{i}{ }^{\mathrm{H}}+N \mathbf{I}_{T}\right) & =\operatorname{det}\left(\left(\gamma_{i} P_{t} \mathbf{S}_{i} \mathbf{S}_{i}{ }^{\mathrm{H}}+\mathbf{I}_{T}\right) N\right) \\
& =N^{T} \operatorname{det}\left(\gamma_{i} P_{t} \mathbf{S}_{i} \mathbf{S}_{i}{ }^{\mathrm{H}}+\mathbf{I}_{T}\right) \\
& =N^{T} \operatorname{det}\left(\gamma_{i} P_{t} \mathbf{S}_{i}{ }^{\mathrm{H}} \mathbf{S}_{i}+\mathbf{I}_{M}\right) \\
& =N^{T} \operatorname{det}\left(\gamma_{i} P_{t} \mathbf{I}_{M}+\mathbf{I}_{M}\right) \\
& =N^{T}\left(\gamma_{i} P_{t}+1\right)^{M}
\end{aligned}
$$

With the formulas $(\mathbf{A}+\mathbf{B C D})^{-1}=\mathbf{A}^{-1}-\mathbf{A}^{-1} \mathbf{B}\left(\mathbf{C}^{-1}+\mathbf{D A}^{-1} \mathbf{B}\right)^{-1} \mathbf{D} \mathbf{A}^{-1}$, $(k \mathbf{A})^{-1}=k^{-1} \mathbf{A}^{-1}$, and $\mathbf{S}_{i}{ }^{-1}=\mathbf{S}_{i}{ }^{\mathrm{H}}$ for unitary matrices, the numerator of 
Equation (D.3) is simplified to [93]:

$$
\begin{aligned}
\left(N \gamma_{i} P_{t} \mathbf{S}_{i} \mathbf{S}_{i}{ }^{\mathrm{H}}+N \mathbf{I}_{T}\right)^{-1} & =\frac{1}{N}\left(\gamma_{i} P_{t} \mathbf{S}_{i} \mathbf{S}_{i}{ }^{\mathrm{H}}+\mathbf{I}_{T}\right)^{-1} \\
& =\frac{1}{N}\left(\mathbf{I}_{T}-\gamma_{i} P_{t}\left(\mathbf{S}_{i}{ }^{-1}+\gamma_{i} P_{t} \mathbf{S}_{i}{ }^{\mathrm{H}}\right)^{-1} \mathbf{S}_{i}{ }^{\mathrm{H}}\right) \\
& =\frac{1}{N}\left(\mathbf{I}_{T}-\gamma_{i} P_{t}\left(\mathbf{S}_{i}{ }^{\mathrm{H}}+\gamma_{i} P_{t} \mathbf{S}_{i}{ }^{\mathrm{H}}\right)^{-1} \mathbf{S}_{i}{ }^{\mathrm{H}}\right) \\
& =\frac{1}{N}\left(\mathbf{I}_{T}-\gamma_{i} P_{t}\left(1+\gamma_{i} P_{t}\right)^{-1} \mathbf{S}_{i} \mathbf{S}_{i}{ }^{\mathrm{H}}\right)
\end{aligned}
$$

Finally, Equation (D.3) has the following expression:

$$
\begin{aligned}
\hat{\imath}_{u, 1} & =\arg \max _{i} \frac{\exp \left(-\frac{1}{N} \operatorname{Tr}\left(\mathbf{\Upsilon}^{\mathrm{H}}\left(\mathbf{I}_{T}-\gamma_{i} P_{t}\left(1+\gamma_{i} P_{t}\right)^{-1} \mathbf{S}_{i} \mathbf{S}_{i}{ }^{\mathrm{H}}\right) \mathbf{\Upsilon}\right)\right)}{\pi^{T N}\left(N^{T}\left(\gamma_{i} P_{t}+1\right)^{M}\right)^{N}} \\
& =\arg \min _{i} \operatorname{Tr}\left(\boldsymbol{\Upsilon}^{\mathrm{H}}\left(\mathbf{I}_{T}-\gamma_{i} P_{t}\left(1+\gamma_{i} P_{t}\right)^{-1} \mathbf{S}_{i} \mathbf{S}_{i}{ }^{\mathrm{H}}\right) \mathbf{\Upsilon}\right) \\
& =\arg \max _{i} \operatorname{Tr}\left(\mathbf{\Upsilon}^{\mathrm{H}}\left(\gamma_{i} P_{t}\left(1+\gamma_{i} P_{t}\right)^{-1} \mathbf{S}_{i} \mathbf{S}_{i}{ }^{\mathrm{H}}\right) \mathbf{\Upsilon}\right) \\
& =\arg \max _{i} \operatorname{Tr}\left(\mathbf{\Upsilon}^{\mathrm{H}} \mathbf{S}_{i} \mathbf{S}_{i}{ }^{\mathrm{H}} \mathbf{\Upsilon}\right)
\end{aligned}
$$




\section{References}

[1] ITU, "ICT Facts and Figures," 2015.

[2] G. de la Roche, A. Alayón-Glazunov, and B. Allen, LTE-Advanced and Next Generation Wireless Networks: Channel Modelling and Propagation, 1st ed. Wiley Publishing, 2012.

[3] ITU-R, "M.2083: IMT Vision Framework and overall objectives of the future development of IMT for 2020 and beyond," ITU, Tech. Rep., 2015.

[4] 3GPP, "RWS-150073: RAN workshop on 5G: Chairman Summary," 2015.

[5] Samsung and Nokia Networks, "RP-151606: New SID Proposal: Study on channel model for frequency spectrum above $6 \mathrm{GHz}$," 3GPP, Tech. Rep., 2015.

[6] G. Liu, F. Yu, H. Ji, V. Leung, and X. Li, "In-band full-duplex relaying: A survey, research issues and challenges," IEEE Communications Surveys \& Tutorial, vol. 17, no. 2, pp. 500-524, Secondquarter 2015.

[7] Z. Ding, I. Krikidis, B. Rong, J. Thompson, C. Wang, and S. Yang, "On combating the half-duplex constraint in modern cooperative networks: protocols and techniques," IEEE Wireless Communications, vol. 19, no. 6, pp. 20-27, December 2012.

[8] K. Doppler, M. Rinne, C. Wijting, C. Ribeiro, and K. Hugl, "Device-todevice communication as an underlay to LTE-Advanced networks," IEEE Communications Magazine, vol. 47, no. 12, pp. 42-49, Dec. 2009.

[9] G. Fodor, E. Dahlman, G. Mildh, S. Parkvall, N. Reider, G. Miklos, and Z. Turanyi, "Design aspects of network assisted device-to-device communications," IEEE Communications Magazine, vol. 50, no. 3, pp. 170-177, Mar. 2012. 
[10] T. Peng, Q. Lu, H. Wang, S. Xu, and W. Wang, "Interference avoidance mechanisms in the hybrid cellular and device-to-device systems," in 20th IEEE International Symposium on Personal, Indoor and Mobile Radio Communications (PIMRC), Sep. 2009, pp. 617-621.

[11] H. Min, J. Lee, S. Park, and D. Hong, "Capacity Enhancement Using an Interference Limited Area for Device-to-Device Uplink Underlaying Cellular Networks," IEEE Transactions Wireless Communications, vol. 10, no. 12 , pp. 3995-4000, Dec. 2011.

[12] P. Janis, V. Koivunen, C. Ribeiro, K. Doppler, and K. Hugl, "Interferenceavoiding MIMO schemes for device-to-device radio underlaying cellular networks," in 20th IEEE International Symposium on Personal, Indoor and Mobile Radio Communications (PIMRC), Sep. 2009, pp. 2385-2389.

[13] P. Janis, C. Yu, K. Doppler, C. Ribeiro, C. Wijting, K. Hugl, O. Tirkonnen, and V. Koivunen, "Device-to-Device Communication Underlaying Cellular Communications Systems," International Journal of Communications, Network and System Sciences, vol. 2, no. 3, pp. 169-178, Jun. 2009.

[14] C.-H. Yu, K. Doppler, C. Ribeiro, and O. Tirkkonen, "Resource Sharing Optimization for Device-to-Device Communication Underlaying Cellular Networks," IEEE Transactions Wireless Communications, vol. 10, no. 8, pp. 2752-2763, Aug. 2011.

[15] C.-H. Yu, O. Tirkkonen, K. Doppler, and C. Ribeiro, "Power Optimization of Device-to-Device Communication Underlaying Cellular Communication," in IEEE International Conference on Communications (ICC), Jun. 2009, pp. 1-5.

[16] C.-H. Yu, O. Tirkkonen, K. Doppler, and C. Ribeiro, "On the Performance of Device-to-Device Underlay Communication with Simple Power Control," in 69th IEEE Vehicular Technology Conference (VTC Spring 2009), Apr. 2009, pp. 1-5.

[17] K. Doppler, C.-H. Yu, C. B. Ribeiro, and P. Janis, "Mode Selection for Device-To-Device Communication Underlaying an LTE-Advanced Network," in IEEE Wireless Communications and Networking Conference, Apr. 2010, pp. 1-6.

[18] M. Zulhasnine, C. Huang, and A. Srinivasan, "Efficient resource allocation for device-to-device communication underlaying LTE network," in 6th IEEE International Conference on Wireless and Mobile Computing, Networking and Communications (WiMob), Oct. 2010, pp. 368-375. 
[19] IEEE 802.21, "IEEE P802.21, Draft Standard for Local and Metropolitan Area Networks: Media Independent Handover Services," 2008.

[20] S. J. Bae, M. Y. Chung, and J. So, "Handover triggering mechanism based on IEEE 802.21 in heterogeneous networks with LTE and WLAN," in International Conference on Information Networking (ICOIN), Jan. 2011, pp. 399-403.

[21] J.-H. Seol and J.-M. Chung, "IEEE 802.21 MIH based Handover for Next Generation Mobile Communication Systems," in 4th International Conference on Innovations in Information Technology (IIT), 2007, pp. 431-435.

[22] D. Bultmann, J. Luo, and E. Schulz, "Media independent handover enabled advanced mobility management and its functional mapping," in International Conference on Communications, Circuits and Systems (ICCCAS), May 2008, pp. 134-138.

[23] I. E. Telatar, "Capacity of multi-antenna Gaussian channels," European Transactions on Telecommunications, vol. 10, pp. 585-595, Dec. 1999.

[24] G. J. Foschini, "Layered space-time architecture for wireless communication in a fading environment when using multi-element antennas," Bell Labs Technical Journal, vol. 1, no. 2, pp. 41-59, Autumn 1996.

[25] L. Zheng and D. Tse, "Diversity and multiplexing: a fundamental tradeoff in multiple-antenna channels," IEEE Transactions on Information Theory, vol. 49, no. 5, pp. 1073-1096, May 2003.

[26] A. Wittneben, "A new bandwidth efficient transmit antenna modulation diversity scheme for linear digital modulation," in IEEE International Conference on Communications (ICC), vol. 3, May 1993, pp. 1630-1634 vol.3.

[27] V. Tarokh, N. Seshadri, and A. Calderbank, "Space-time codes for high data rate wireless communication: performance criterion and code construction," IEEE Transactions on Information Theory, vol. 44, no. 2, pp. 744-765, Mar. 1998.

[28] S. Alamouti, "A simple transmit diversity technique for wireless communications," IEEE Journal on Selected Areas in Communications, vol. 16, no. 8, pp. 1451-1458, Oct. 1998.

[29] V. Tarokh, H. Jafarkhani, and A. Calderbank, "Space-time block codes from orthogonal designs," IEEE Transactions on Information Theory, vol. 45, no. 5, pp. 1456-1467, Jul. 1999. 
[30] B. Hassibi and B. M. Hochwald, "How Much Training is Needed in Multiple-Antenna Wireless Links?" IEEE Transactions on Information Theory, vol. 49, no. 2, pp. 951-963, Apr. 2003.

[31] T. L. Marzetta and B. M. Hochwald, "Capacity of a mobile multipleantenna communication link in Rayleigh flat fading," IEEE Transactions on Information Theory, vol. 45, pp. 139-157, Jan. 1999.

[32] B. M. Hochwald and T. L. Marzetta, "Unitary space-time modulation for multiple-antenna communications in Rayleigh flat fading," IEEE Transactions on Information Theory, vol. 46, no. 2, pp. 543-564, Mar. 2000.

[33] L. Zheng and D. N. C. Tse, "Communication on the Grassmann manifold: a geometric approach to the noncoherent multiple-antenna channel," IEEE Transactions on Information Theory, vol. 48, no. 2, pp. 359-383, Feb. 2002.

[34] R. H. Gohary and T. N. Davidson, "Noncoherent MIMO Communication: Grassmannian Constellations and Efficient Detection," IEEE Transactions on Information Theory, vol. 55, no. 3, pp. 1176-1205, Mar. 2009.

[35] B. Hochwald and W. Sweldens, "Differential Unitary Space-Time Modulation," IEEE Transactions on Communications, vol. 48, no. 12, pp. 20412052, Dec. 2000.

[36] B. Hassibi and B. M. Hochwald, "Cayley differential unitary space-time codes," IEEE Transactions on Information Theory, vol. 48, no. 6, pp. 1485-1503, Jun. 2002.

[37] Y. Yu, G. Giannakis, and N. Jindal, "Information-Bearing Noncoherently Modulated Pilots for MIMO Training," IEEE Transactions on Information Theory, vol. 53, no. 3, pp. 1160-1168, Mar. 2007.

[38] "FP7 European Project 317669 METIS (Mobile and wireless communications Enablers for the Twenty-twenty Information Society)," https://www.metis2020.com/, 2012.

[39] J. F. Monserrat, G. Mange, V. Braun, H. Tullberg, G. Zimmermann, and O. Bulakci, "METIS research advances towards the $5 \mathrm{G}$ mobile and wireless system definition," EURASIP Journal on Wireless Communications and Networking, vol. 2015, no. 1, 2015.

[40] 3GPP, "TR 36.806: Relay architectures for E-UTRA (LTE-Advanced) (Release 9)," 2010. 
[41] 3GPP, "TR 36.814: Further advancements for E-UTRA physical layer aspects (Release 9)," 2010.

[42] 3GPP, "TS 36.416: Mobile Relay for EUTRA (Release 11)," 2011.

[43] 3GPP, "TR 36.843: Study on LTE Device to Device Proximity Services; Radio Aspects (Release 12)," 2014.

[44] 3GPP, "TR 22.803: Feasibility study for Proximity Services (ProSe) (Release 12)," 2013.

[45] Qualcomm, "Creating a Digital 6th Sense with LTE Direct," 2014.

[46] ITU-R, "M.2135 Guidelines for evaluation of radio interface technologies for IMT-Advanced," ITU, Tech. Rep., 2008.

[47] InterDigital, "R1-132186: On the use of UL or DL radio resources for D2D," 3GPP, Tech. Rep., 2013.

[48] ETSI TC ITS, "Reply LS on information regarding V2X study in 3GPP," 2015.

[49] 3GPP SA WG1, "SP-150180: LS on V2X Study in 3GPP SA1," 2015.

[50] 3GPP, "TR 22.885: Study on LTE Support for V2X Services (Release 14)," 2015.

[51] 3GPP, "TR 25.924: Opportunity Driven Multiple Access," 1999.

[52] D. Martín-Sacristán, J. F. Monserrat, J. Cabrejas-Peñuelas, D. Calabuig, S. Garrigas, and N. Cardona, "On the way towards fourth-generation mobile: 3GPP LTE and LTE-Advanced," EURASIP Journal on Wireless Communications and Networking, vol. 2009, pp. 4:1-4:10, Mar. 2009.

[53] 3GPP, "TR 36913: Requirements for further advancements for Evolved Universal Terrestrial Radio Access (E-UTRA) (LTE-Advanced) (Release 10)," 2011.

[54] IEEE 802.16j, "IEEE Standard for Local and metropolitan area networks Part 16: Air Interface for Broadband Wireless Access Systems, Amendment 1: Multi-hop Relay Specication," 2009.

[55] ITU-R, "Requirements, evaluation criteria and submission templates for the development of IMT-Advanced," ITU, Tech. Rep., 2008. 
[56] IEEE 802.11n, "Part 11: Wireless LAN Medium Access Control (MAC) and Physical Layer (PHY) Specications. Amendment 5: Enhancements for Higher Throughput," 2009.

[57] 3GPP, "TS 36.355: LTE Positioning Protocol (LPP)," 2015.

[58] 3GPP, "TS 36.455: LTE Positioning Protocol A (LPPa)," 2011.

[59] "Celtic Project CP5-026 Wireless World Initiative New Radio +(WINNER+) ," http://projects.celtic-initiative.org/winner+/, 2008.

[60] WINNER+, "Doc. IMTADV/ 22: Final evaluation report from Winner+ on the IMT-Advanced proposal in Documents IMT-ADV/6, IMT-ADV/8 and IMT-ADV/9," WINNER+, Tech. Rep., 2010.

[61] D. Green and A. Obaidat, "An accurate line of sight propagation performance model for ad-hoc 802.11 wireless LAN (WLAN) devices," in IEEE Conference on Communications (ICC), vol. 5, 2002, pp. 3424-3428 vol.5.

[62] D. B. Johnson and D. A. Maltz, "Dynamic Source Routing in Ad Hoc Wireless Networks," in Mobile Computing, ser. The Kluwer International Series in Engineering and Computer Science, T. Imielinski and H. F. Korth, Eds. Springer US, 1996, vol. 353, pp. 153-181.

[63] D. Martín-Sacristán, J. Monserrat, D. Calabuig, and N. Cardona, "TimeFrequency Coupled Proportional Fair Scheduler with Multicarrier Awareness for LTE Downlink," in 81st IEEE Vehicular Technology Conference (VTC), May 2015, pp. 1-5.

[64] W. Forum, "A Comparative Analysis of Spectrum Alternatives for WiMAX Networks with Deployment Scenarios Based on the U.S. $700 \mathrm{MHz}$ Band." WiMAX Forum, Tech. Rep., 2008.

[65] K. Johansson, A. Furuskar, P. Karlsson, and J. Zander, "Relation between base station characteristics and cost structure in cellular systems," in 15th IEEE International Symposium on Personal, Indoor and Mobile Radio Communications (PIMRC), vol. 4, Sep. 2004, pp. 2627-2631 Vol.4.

[66] B. C. Forge S. and B. E., "The demand for future mobile communications markets and services in Europe." IPTS Technical Report Prepared for the European Commission - Joint Research Centre, Tech. Rep., 2005.

[67] O. Elijah, C. Leow, T. Rahman, S. Nunoo, and S. Iliya, "A Comprehensive Survey of Pilot Contamination in Massive MIMO - 5G System," IEEE Communications Surveys $\mathcal{E}$ Tutorial, 2015. 
[68] S. Sesia, I. Toufik, and M. Baker, LTE The UMTS Long Term Evolution From Theory to Practice. Great Britain: John Wiley, 2011.

[69] T. L. Marzetta, "BLAST Training: Estimating Channel Characteristics for High Capacity Space-Time Wireless," in 37th Annual Allerton Conference on Communications, Control, and Computing, 1999, pp. 958-966.

[70] Y. R. Zheng and C. Xiao, "Improved models for the generation of multiple uncorrelated Rayleigh fading waveforms," IEEE Communications Letters, vol. 6 , no. 6 , pp. 256-258, Jun. 2002.

[71] B. Hochwald and W. Sweldens, "Differential unitary space-time modulation," IEEE Transactions on Communications, vol. 48, no. 12, pp. 20412052, Dec. 2000.

[72] M. Beko, J. Xavier, and V. Barroso, "Noncoherent Communication in Multiple-Antenna Systems: Receiver Design and Codebook Construction," IEEE Transactions on Signal Processing, vol. 55, no. 12, pp. 57035715, Dec. 2007.

[73] H. Jafarkhani, "A quasi-orthogonal space-time block code," IEEE Transactions on Communications, vol. 49, no. 1, pp. 1-4, Jan. 2001.

[74] J. C. Belfiore, G. Rekaya, and E. Viterbo, "The Golden code: a 2x2 fullrate space-time code with nonvanishing determinants," IEEE Transactions on Information Theory, vol. 51, no. 4, pp. 1432-1436, 2005.

[75] Y. M. M. Fouad, R. H. Gohary, J. Cabrejas, H. Yanikomeroglu, D. Calabuig, S. Roger, and J. F. Monserrat, "Time-Frequency Grassmannian Signalling for MIMO Multi-Channel-Frequency-Flat Systems," IEEE Wireless Commun. Letters, vol. 19, no. 3, pp. 475-478, Mar. 2015.

[76] J. G. Proakis and M. Salehi, Digital Communications. New York: Mc Graw-Hill, 2008.

[77] T. Beitelmal, R. Schoenen, and H. Yanikomeroglu, "On the impact of correlated shadowing on the performance of user-in-the-loop for mobility," in IEEE International Conference on Communications (ICC), June 2012, pp. 7040-7044.

[78] 3GPP, "TS 36.101: User Equipment (UE) radio transmission and reception (Release 12)," 2014. 
[79] D. Martín-Sacristán, J. Cabrejas, D. Calabuig, and J. F. Monserrat, "MAC Layer Performance of Different Channel Estimation Techniques in UTRAN LTE Downlink," in 69th IEEE Vehicular Technology Conference (VTC Spring 2009), Apr. 2009, pp. 1-5.

[80] T. Cover, "Broadcast channels," IEEE Transactions on Information Theory, vol. 18, no. 1, pp. 2-14, Jan. 1972.

[81] S. Vanka, S. Srinivasa, Z. Gong, P. Vizi, K. Stamatiou, and M. Haenggi, "Superposition Coding Strategies: Design and Experimental Evaluation," IEEE Transactions Wireless Communications, vol. 11, no. 7, pp. 26282639, Jul. 2012.

[82] I. Kammoun, A. Cipriano, and J. Belfiore, "Non-Coherent Codes over the Grassmannian," IEEE Transactions Wireless Communications, vol. 6, no. 10, pp. 3657-3667, Oct. 2007.

[83] NTT DoCoMo, Inc., "5G Radio Access: Requirements, Concept and Technologies," DoCoMo 5G White Paper, Jul. 2014.

[84] Z. Shen, A. Papasakellariou, J. Montojo, D. Gerstenberger, and F. Xu, "Overview of 3GPP LTE-Advanced carrier aggregation for 4G wireless communications," IEEE Communications Magazine, vol. 50, no. 2, pp. 122-130, Feb. 2012.

[85] 3GPP, "TS 36.213: Physical layer procedures (Release 8)," 2010.

[86] A. M. Law and D. M. Kelton, Simulation Modeling and Analysis, 3rd ed. McGraw-Hill Higher Education, 1999.

[87] 3GPP, "TS 36.212: Multiplexing and channel coding (Release 8)," 2010.

[88] 3GPP, "TS 36.211: Physical channels and modulation (Release 12)," 2015.

[89] 3GPP, "TR 25.943: Deployment aspects (Release 8)," 2009.

[90] J. Kermoal, L. Schumacher, K. Pedersen, P. Mogensen, and F. Frederiksen, "A stochastic MIMO radio channel model with experimental validation," IEEE Journal on Selected Areas in Communications, vol. 20, no. 6, pp. 1211-1226, Aug. 2002.

[91] M. S. Akram, "Pilot-based Channel Estimation in OFDM systems," Master's thesis, Nokia Mobiles Phones, 2007. 
[92] A. M. Cipriano, "Algorithmes de Décodage pour des Codes EspaceTemps Non Cohérents," Ph.D. dissertation, École Nationale Supérieure des Télécommunications, 2005.

[93] R. A. Horn and C. R. Johnson, Eds., Matrix Analysis. New York, NY, USA: Cambridge University Press, 1986. 
